\title{
Examining the effects of acute stress on memory in eyewitness settings
}

Citation for published version (APA):

Marr, C. (2021). Examining the effects of acute stress on memory in eyewitness settings. [Doctoral Thesis, Maastricht University, University of Portsmouth]. The House of Legal Psychology. https://doi.org/10.26481/dis.20211110cm

Document status and date:

Published: 01/01/2021

DOI:

$10.26481 /$ dis. $20211110 \mathrm{~cm}$

Document Version:

Publisher's PDF, also known as Version of record

\section{Please check the document version of this publication:}

- A submitted manuscript is the version of the article upon submission and before peer-review. There can be important differences between the submitted version and the official published version of record.

People interested in the research are advised to contact the author for the final version of the publication, or visit the DOI to the publisher's website.

- The final author version and the galley proof are versions of the publication after peer review.

- The final published version features the final layout of the paper including the volume, issue and page numbers.

Link to publication

\footnotetext{
General rights rights.

- You may freely distribute the URL identifying the publication in the public portal. please follow below link for the End User Agreement:

www.umlib.nl/taverne-license

Take down policy

If you believe that this document breaches copyright please contact us at:

repository@maastrichtuniversity.nl

providing details and we will investigate your claim.
}

Copyright and moral rights for the publications made accessible in the public portal are retained by the authors and/or other copyright owners and it is a condition of accessing publications that users recognise and abide by the legal requirements associated with these

- Users may download and print one copy of any publication from the public portal for the purpose of private study or research.

- You may not further distribute the material or use it for any profit-making activity or commercial gain

If the publication is distributed under the terms of Article $25 \mathrm{fa}$ of the Dutch Copyright Act, indicated by the "Taverne" license above, 


\section{Examining the Effects of Acute Stress on Memory in Eyewitness Settings}

Carey Marr 
Cover design: Hailey Herring-Newbound | www.haileyherringnewbound.com Lay-out: $\quad$ Publiss | www.publiss.nl

Print: $\quad$ Ridderprint $\mid$ www.ridderprint.nl

(C) Copyright 2021: Carey Marr, Maastricht, The Netherlands.

All rights reserved. No part of this publication may be reproduced, stored in a retrieval system, or transmitted in any form or by any means, electronic, mechanical, by photocopying, recording, or otherwise, without the prior written permission of the author. 


\title{
Maastricht University
}

\section{Examining the Effects of Acute Stress on Memory in Eyewitness Settings}

\author{
DISSERTATION \\ to obtain the degree of Doctor of Philosophy from The University \\ of Portsmouth and the degree of Doctor at Maastricht University, \\ on the authority of the Rector Magnificus, Prof. dr. Rianne M. Letschert \\ in accordance with the decision of the Board of Deans, \\ to be defended in public
}

on Wednesday, 10th of November at 10:00

by

Carey Marr

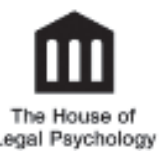




\section{Supervisors:}

Prof. dr. Henry Otgaar

Prof. dr. Lorraine Hope
KU Leuven, Belgium \& Maastricht University, the Netherlands University of Portsmouth, United Kingdom

\section{Co-supervisors:}

Dr. Melanie Sauerland Maastricht University, the Netherlands

Dr. Conny Quaedflieg Maastricht University, the Netherlands

\section{Assessment Committee:}

Prof. dr. Marko Jelicic (chair) Maastricht University, the Netherlands

Dr. Lucy Akehurst University of Portsmouth, United Kingdom

Prof. dr. Arjan Blokland Maastricht University, the Netherlands

Dr. Ana Gheorghiu University of Portsmouth, United Kingdom

Prof. dr. Tom Smeets Tilburg University, the Netherlands

Dr. Melanie Takarangi Flinders University, Australia

This research was supported by a fellowship from the Erasmus Mundus Joint Doctorate Program: The House of Legal Psychology (EMJD-LP) with Framework Partnership Agreement (FPA) 2013-0036 and Specific Grant Agreement (SGA) 532473-EM-52017-1-NL-ERA MUNDUS-EPJD to Carey Marr. 


\section{Table of Contents}

Chapter 1 General Introduction 1

Chapter 2 Memory Experts' and Laypeople's Beliefs about the Effects of 33 Acute Stress on (Eyewitness) Memory Performance

Chapter 3 Examining the Effects of Acute Stress at Encoding and Retrieval 71 on Face Recognition Memory

Chapter 4 Mitigating the Negative Effects of Retrieval Stress on Memory: 101 Examining the Efficacy of an Arousal Reappraisal Intervention

$\begin{array}{lll}\text { Chapter } 5 \text { General Discussion } & 131\end{array}$

$\begin{array}{ll}\text { References } & 151\end{array}$

$\begin{array}{ll}\text { Summary } & 165\end{array}$

$\begin{array}{ll}\text { Acknowledgements } & 167\end{array}$

$\begin{array}{ll}\text { Dissemination } & 168\end{array}$

$\begin{array}{ll}\text { Curriculum Vitae } & 169\end{array}$

$\begin{array}{ll}\text { Valorisation Addendum } & 170\end{array}$

$\begin{array}{ll}\text { Appendices } & 172\end{array}$ 


\section{CHAPTER 1}

\section{General Introduction}

This chapter draws from the following manuscripts:

Marr, C., Sauerland, M., Otgaar, H., Quaedflieg, C.W.E.M., \& Hope, L. (2018). The effect of acute stress on memory: How it helps and how it hurts. In Mind, 9(38), 1. https://www.in-mind.org/article/the-effect-of-acute-stress-on-memoryhow-it-helps-and-how-it-hurts

Marr, C., Sauerland, M., Otgaar, H., Quaedflieg, C.W.E.M., \& Hope, L. (2021). The effects of acute stress on eyewitness memory: An integrative review for eyewitness researchers. Memory, 29(8), 1091-1100. https://doi.org/10.1080/09658211.2021.1955935 


\section{Introduction}

Paula is taking a walk in the park when she hears a loud scream. She turns a corner and sees a woman on the ground with a man standing over her. Paula watches as the man kicks and punches the woman multiple times. Paula notices that her heartbeat quickens, her body tenses, and her palms are sweaty. Before she can make a decision about what to do, the man looks up and sees Paula. He turns and runs off in the opposite direction. Paula runs towards to woman to check on her and immediately calls the police, who soon arrive at the crime scene. A few days later, the police call Paula into the station for an interview and an identification procedure. The police are considerate, but she is incredibly tense throughout the process, again feeling her palms sweaty and her heart race. She thinks she recognizes the perpetrator, and selects his picture from the lineup. A few months later, in the courtroom, she gives testimony against the man she chose from the lineup, describing to the jury what she saw on the day of the crime. Although she was not the victim of the crime, Paula experienced acute stress at the scene of the crime, as well as later when being interviewed at the police station and when testifying in the courtroom.

In circumstances such as those experienced by this (fictitious) witness, it is important to consider how stress experienced at both encoding and retrieval may affect ability to identify a perpetrator and remember details about the crime incident. Understanding how acute stress can affect memory is vital for courts to assess the accuracy and reliability of eyewitness testimony concerning a crime. Furthermore, knowing how stress impacts recognition and recall performance is crucial for developing tools (e.g., interview protocols) that can aid police officers when conducting interviews with victims, witnesses, or suspects.

Memory experts' and laypeople's beliefs about the stress-memory relationship are largely unknown, despite the fact that these beliefs can influence legal decisions about credibility and guilt in the courtroom (Bornstein et al., 2008). Although decades of research have examined how stress may affect eyewitness memory identifications and reports (e.g., Christianson, 1992; Deffenbacher et al., 2004; Echterhoff \& Wolf, 2012; Sauerland et al., 2016), studies examining how acute stress at encoding and retrieval can affect memory performance have produced inconsistent findings (e.g., Deffenbacher et al., 2004; Shields et al., 2017). Different research lines examining the effects of acute stress on memory each have methodological limitations. On the one hand, past work addressing more applied eyewitness questions has often overlooked critical components 
of stress effects on memory. For example, little work has examined stress at retrieval in eyewitness contexts (but see Dellapaolera, 2019; Robicheaux, 2016), despite the fact that fundamental memory researchers have identified negative effects of acute retrieval stress on memory performance (e.g., Het et al., 2005; Kuhlmann et al., 2005a; Lupien et al., 2009; Wolf, 2017). Furthermore, eyewitness research has rarely properly considered the specific time course of the physiological stress response (as discussed by Bornstein $\&$ Robicheaux, 2009; Sauerland et al., 2016; Thomas \& Karanian, 2019). On the other hand, fundamental memory research does not typically use stimuli relevant to eyewitness situations (e.g., faces), but rather relies on stimuli that are a far stretch from the events that eyewitnesses typically experience (e.g., word lists, static pictures; Schwabe et al., 2008a; Smeets et al., 2007; Zoladz et al., 2011). Finally, few studies from either research field have explored how to mitigate negative effects of stress experienced during retrieval on recall and recognition performance. Testing interventions that could be applied in police interviews would be valuable.

The overarching goal of this thesis is to investigate the relationship between acute stress and memory in eyewitness contexts. This work aimed to combine the distinct theoretical and methodological strengths of the eyewitness and fundamental memory fields to enhance understanding of the topic. Through experimental research, I sought to fill some of the present gaps in this literature. First, I addressed the dearth of knowledge about experts' and layperson's beliefs regarding stress effects on (eyewitness) memory performance by examining current beliefs in a survey (Chapter 2, Experiment 1). Second, I confronted the lack of existing experiments using contemporary, rigorous methodology to investigate effects of encoding and retrieval stress on the recognition of eyewitness-relevant stimuli. To do so, I used validated procedures to test stress effects in a face recognition task (Chapter 3, Experiment 2), with a specific focus on stress effects at encoding (Chapter 3, Experiment 3). Finally, I shifted focus to stress experienced at retrieval, as few studies have examined ways to reduce negative effects of retrieval stress on memory performance. To begin filling this gap, I assessed the efficacy of an arousal reappraisal intervention to mitigate potential negative effects of stress on recognition and free recall performance (Chapter 4, Experiment 4).

In this introductory chapter, I will place the current thesis in context by (1) examining how beliefs about the effects of acute stress on eyewitness memory performance can play a role in real life cases, (2) reviewing the neurobiological underpinnings and theoretical premises regarding stress effects on memory recognition and recall, (3) examining the 
current state of the substantial literature regarding encoding stress and retrieval stress effects on memory, and (4) identifying ongoing gaps in this existing knowledge base.

\section{Beliefs about the Effects of Acute Stress on Memory}

An important starting point and, indeed, rationale for this thesis is to recognize how beliefs about the stress-memory relationship affect real life decision making in eyewitness contexts. Specifically, understanding what memory experts and laypeople believe about this intricate relationship is vital in eyewitness contexts, because both of these groups can bring their beliefs into the courtroom. For example, memory researchers may be asked to serve as expert witnesses in a case, sharing their understanding of the research base with the judge and jury (e.g., Kassin et al., 1989). Laypeople acting as jurors may evaluate eyewitness evidence based on their commonsense beliefs. For example, the commonsense model of jury decision making (Groscup \& Talon, 2009) suggests that jurors often rely on their commonsense beliefs, which becomes problematic when lay understanding of a situation is not accurate. Indeed, research suggests that juror beliefs regarding stress effects on memory performance can and do influence juror's decisions about credibility and guilt (Bornstein et al., 2008).

Few studies have investigated experts' beliefs about the effects of stress on memory performance. The three existing surveys examining experts' beliefs used the same single statement (Very high levels of stress impair the accuracy of eyewitness testimony; Kassin et al., 1989; Kassin et al., 2001; Yarmey \& Jones, 1983). These surveys showed varied levels of endorsement, with around $60 \%$ to $88 \%$ of expert respondents endorsing the statement as reliable enough for psychologists to present in court. However, such a statement is too general to capture the complex effects of the stress-memory relationship and fails to specify the memory phase (i.e., encoding vs. retrieval) to which such effects might apply. In recent decades, researchers have examined the effects of the particularities of stressor timing on distinct memory phases, resulting in new models delineating more precise timelines of how stress affects memory performance (e.g., Diamond et al., 2007; Joëls et al., 2006). Thus, it is important to capture a more fine-grained perspective of experts' understanding with respect to a broader set of related matters, such as stressor timing, neuroscientific bases, and potential moderators of the stress-memory relationship. Additionally, the previous surveys were conducted nearly over two decades ago, and many studies regarding stress and memory performance have been published since then: a recent meta-analysis assessing the acute stress-memory relationship included 90 papers, and all 
had been published during or after 2001 (Shields et al., 2017). Therefore, contemporary experts' beliefs about acute stress effects on memory performance, particularly in eyewitness contexts, are largely unknown.

A greater number of surveys have investigated laypeople's beliefs on this topic over the years, usually using the same single statement as in the previous expert surveys (e.g., Benton et al., 2006; Deffenbacher \& Loftus, 1982; Kassin \& Barndollar, 1992; Read \& Desmarais, 2009). Varying levels of endorsement have also been found, ranging from $42 \%$ endorsement (Deffenbacher \& Loftus, 1982) to 92\% endorsement (Read \& Desmarias, 2009), with no particular pattern of increases or decreases in consensus over the years. Contemporary laypeople's commonsense beliefs about the stress-memory relationship and specific relevant factors in eyewitness settings are therefore similarly unknown.

In sum, beliefs about the stress-memory relationship can be powerful in eyewitness contexts, regardless of what contemporary scientific research about the effects of acute stress on memory suggests. In the absence of expert testimony, laypeople likely use their commonsense beliefs to evaluate eyewitness evidence. Even with expert testimony, two opposing experts could present conflicting opinions in court, interpreting the body of research in a different way. In this case, contradictory information could create even more confusion on the topic for a judge and jury. Therefore, understanding current experts' and layperson's beliefs about stress effects on memory performance has great practical implications for the courtroom.

\section{Neurobiological Underpinnings of Stress Effects on Memory}

To understand the effects of stress on memory from a scientific perspective, it is imperative to know how the brain responds to stressful situations. There are several stages of physiological response to acute stress (see Figure 1.1). Within seconds of experiencing acute stress, the autonomic nervous system is activated, releasing catecholamines such as adrenaline, noradrenaline, and dopamine (Everly \& Lating, 2013; Nelson \& Kriegsfeld, 2016; Ulrich-Lai \& Herman, 2009). This release leads to typical symptoms of acute stress, such as increased heart rate, blood pressure, and sweating while also enhancing arousal and alertness (Clark et al., 1987; Quaedflieg \& Schwabe, 2018; Robbins, 1984). These acute stress reactions lead to what has been described as the fight-flight-or-freeze response (Cannon, 1929; Barlow, 2002), an automatic survival instinct in which the body prepares to face the threat by either fighting back, running away, or freezing. The released catecholamines also affect several brain areas, including the prefrontal cortex (PFC), the 
amygdala, and the hippocampus. This autonomic activation increases the connectivity between such brain regions by shifting from an executive control network (which focuses on higher-order cognitive functions) towards a salience network (which focuses on cognitive processing related to salient stimuli; Hermans et al., 2011, 2014; Young et al., 2017). The brain areas affected by stress-induced catecholamines are also the main brain areas essential for memory processes, including recognition and recall.

\section{Figure 1.1}

Standard Timeline of a Physiological Acute Stress Response

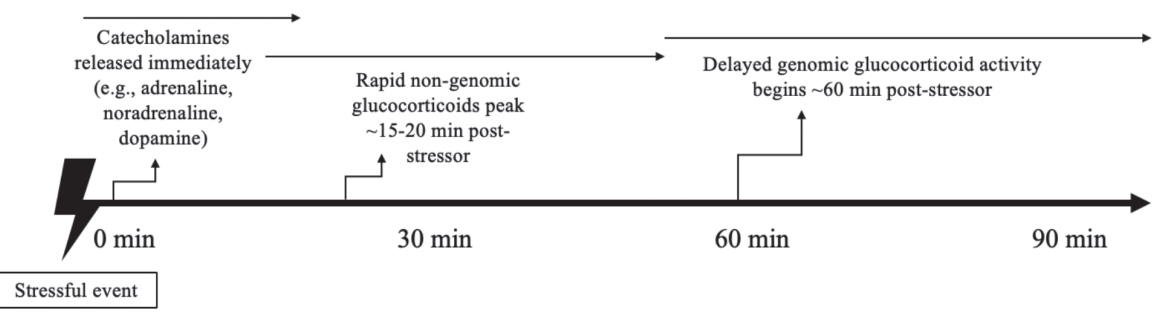

Besides the immediate autonomic response, other neurobiological reactions to acute stress also affect memory formation and retrieval. Specifically, acute stress results in a release of glucocorticoids, particularly cortisol, the main human stress hormone, via the activation of the hypothalamic-pituitary-adrenal (HPA) axis (de Kloet et al., 2005; Everly \& Lating, 2013; Nelson \& Kriegsfeld, 2016; Ulrich-Lai \& Herman, 2009). The effect of cortisol on the brain follows two phases: (1) a rapid non-genomic phase, in which the effects peak about 15-20 minutes after the stressor and continue for around one hour, and (2) a delayed genomic phase, in which the effects begin 60-90 minutes after the stressor (Joëls \& Baram, 2009; Joëls et al., 2011; Quaedflieg \& Schwabe, 2018). These glucocorticoids, released from the adrenal gland, bind to two types of intracellular receptors, mineralocorticoid receptors and glucocorticoid receptors (Joëls et al., 2006). Mineralocorticoid receptors retain high saturation (i.e., a large number of glucocorticoids generally bind to them), while glucocorticoid receptor saturation depends on heightened cortisol, with high saturation during times of cortisol peak (e.g., in the early morning, $67-74 \%$ saturation, Lupien et al., 2007). That is, glucocorticoid receptors specifically become occupied when glucocorticoid levels surge above standard levels in the brain (i.e., after experiencing acute stress). Under these conditions, glucocorticoid receptors initiate a negative feedback response, promoting recovery by suppressing the previously 
stimulated HPA response. These cortisol receptors are abundant in the limbic system and linked brain structures, including the hippocampus, amygdala, and PFC (Karst et al., 2010; Kim et al., 2015). Thus, as with the catecholamine activity, cortisol activity can influence (emotional) memory encoding and retrieval, which primarily rely on these same brain structures.

The independent effects of catecholamines and glucocorticoids can also interact when stressful situations arise. Specifically, when they act in tandem-at the same time and on the same brain structures - their actions have an additive effect, and this interaction results in a united response to stress (Joëls et al., 2006). Indeed, as described by Joëls and colleagues (2006), "This 'stress neuro-symphony' provides the capacity to generate precise, focused alterations in neuronal activity in response to stress signals." (p. 10), which can occur in brain structures such as in the amygdala (Roozendaal \& McGaugh, 2011). However, disruptions in this interaction (e.g., in timing) can result in differing effects, suppressing rather than enhancing each other (Joëls et al., 2011), suggesting the complexity of timing effects of catecholamines, glucocorticoids, and other relevant hormones on memory functioning.

Thus, how stress affects memory performance (i.e., positively or negatively) depends on the specific timing of the stages of acute stress. Also important for understanding the relationship between acute stress and memory performance is to consider memory phase. The first two phases, encoding and consolidation, occur during memory formation. In encoding, information is processed and categorized, before being transferred from shortterm memory into long-term memory and stabilized in the consolidation stage. The third phase of memory is retrieval, in which previously encoded and stored events or information are accessed.

\section{Historical Development of Stress-Memory Models}

Researchers have proposed several different hypotheses about the effects of acute stress on memory performance. Earlier models were based on behavioral examinations of cognitive performance at different levels of arousal and on different types of tasks. These models focused on how physiological arousal affects memory performance, and they were influential in laying the groundwork for research on the effects of acute stress on memory performance. Although my thesis explicitly concentrates on acute stress (i.e., the physiological and psychological experience of an immediate perceived threat), arousal (i.e., one's physiological and psychological state of alertness) is a critical element 
of stress. Moreover, the terms arousal and stress are often used interchangeably in past work (see Dellapaolera, 2019). Finally, though these historical models lack the nuance of contemporary models focusing on neurobiological stress-memory research, examining early models allows a more complete understanding of some key factors in the stressmemory relationship, such as stress severity and type of experienced detail (i.e., central vs. peripheral).

\section{Yerkes-Dodson Law}

The Yerkes-Dodson law, formulated in the early $20^{\text {th }}$ century, posits different effects of high stress on memory performance based on task difficulty (Yerkes \& Dodson, 1908). For simple tasks, this law suggests a linear relationship between arousal and performance, with arousal generally enhancing performance, though plateauing at high levels of arousal. For complex tasks, however, the Yerkes-Dodson law assumes an inverted U relationship, where medium levels of arousal enhance performance, but low or high levels impair performance. During the latter half of the $20^{\text {th }}$ century, interpretations of this law have focused on the curvilinear interpretation, often ignoring the nuance of the original findings (see Diamond et al., 2007, for a detailed historical review). One difficulty in accurate analysis of the Yerkes-Dodson law is differentiating between classifying and defining simple and complex tasks. In recent years, Diamond and colleagues (2007) have suggested examining PFC engagement to help make this distinction, that is, that tasks requiring PFC involvement would be classified as complex tasks under the YerkesDodson law. Indeed, some support for the inverted U relationship has been found when examining working memory, which relies on PFC functioning (e.g., Schoofs et al., 2009; Shields et al., 2016), but also on certain tasks requiring attention or decisionmaking (e.g., Ashcraft, 2002; Aston-Jones et al., 2000). Other findings examining stress and memory performance also support the inverted $U$ curve posited by the YerkesDodson law (e.g., Abercrombie et al., 2003; Andreano \& Cahill, 2006; Het et al., 2005; Metcalfe et al., 2019). However, the Yerkes-Dodson law is often seen as antiquated. In a review on emotional stress and eyewitness memory, Christianson (1992) deemed the model to be unidimensional and non-falsifiable, because the results of all studies could fit the model regardless of the found effects of stress (i.e., impairing or enhancing) on memory performance. Precisely, he stated, "the Yerkes-Dodson law has served out its role in psychology and is no longer needed as a general description in relating emotion and memory in the eyewitness literature." (p. 298). This opinion is echoed in other reviews 
of stress and memory performance (Deffenbacher, 1994; Robinson, 2018; Staal, 2004).

\section{Cue Utilization Hypothesis}

Easterbrook's cue utilization hypothesis (1959) similarly suggests that arousal effects on performance depend on task complexity. The idea behind this model is that arousal reduces the number of cues to which one is able to attend. This reduction of cues can assist performance for simple tasks, where only one or few cues are important. On the other hand, arousal can impair performance for complex tasks, due to this reduction of attention towards a greater number of cues. Thus, cue utilization also helps explain why central information may be better remembered in contrast to peripheral details in stressful situations (Christianson, 1992). Easterbrook did not provide a specific definition on what central and peripheral information constitutes. That is, Easterbrook defined central as elements that were immediately relevant to a task and peripheral as elements that were occasionally or partially relevant. Researchers have interpreted centrality as a spatial matter (i.e., foreground vs. background; e.g., Christianson \& Loftus, 1991) or in regard to relevance to the plot (i.e., relevant vs. irrelevant; e.g., Heuer \& Reisberg, 1990; Wessel et al., 2000), though there is still no overall consensus as to what counts as central and peripheral. In regard to eyewitness situations, central details have been defined or exemplified as a significant element of the witnessed crime (vs. irrelevant elements, Houston et al., 2013), items physically carried or touched by a target (vs. items present but not used, Echterhoff \& Wolf, 2012; Herten et al., 2017), or other crimerelated details such as the perpetrator or weapons that are present (vs. identities of other eyewitnesses, for example, Wulff \& Thomas, 2021).

This model is complementary to the Yerkes-Dodson law, for both suggest similar memory outcomes depending on task difficulty (Diamond et al., 2007). Research generally supports the cue utilization hypothesis, suggesting that attentional narrowing during a stressful experience limits the range of cues to which one is able to attend (e.g., Staal, 2004). The weapon focus effect provides a good example of this attentional effect and its subsequent effects on memory performance (e.g., Fawcett et al., 2016; Kramer et al., 1990; Loftus et al., 1987). The weapon focus effect is a phenomenon whereby eyewitness memory for faces and other details is poorer if a weapon was present during the crime (Fawcett et al., 2013). One explanation of the effect suggests that witnesses focus on the weapon at a cost to all other (less central) cues, illustrating the cue utilization hypothesis (Diamond et al., 2007). Indeed, other work also shows support for Easterbrook's cue 
utilization hypothesis (1959), suggesting enhanced memory for central details but impaired memory for peripheral details in stressful situations (e.g., Christianson \& Loftus, 1987; Heuer \& Reisberg, 1990, but see Lanciano \& Curci, 2011). Still, this early model does not capture some essential elements of modern stress-memory relationship models, such as stressor timing and memory phase.

\section{Summary of Historical Models}

These early models about arousal and memory performance were important starting points for stress research (Diamond et al., 2007; Robinson, 2018). However, they are somewhat simplistic and rudimentary in light of recent neurobiological research as they focus solely on autonomic arousal, and cannot fully explain the subtleties of the stress-memory relationship. Still, the Yerkes-Dodson law and the cue utilization hypothesis highlight factors that may influence the effects of stress on memory performance, such as stressor severity, task difficulty, and detail type. In applied eyewitness situations, these factors likely vary; however, differences in the degree of stress on memory performance have not been systematically examined in the more contemporary models, for example. Therefore, these early models highlight important factors to consider when assessing the potential effects of acute stress on memory.

\section{Current Models of Stress and Memory}

Contemporary models accounting for the effects of acute stress on memory performance have emerged from a new shift in research examining the neurobiological effects of stress on cognitive performance. Understanding these fundamentals is essential when investigating further nuanced questions about stress and memory in eyewitness contexts. Indeed, the work in my thesis primarily stems from ideas proposed by these modern models. In this section, I will first individually introduce the primary models and then jointly evaluate current empirical support.

\section{Dual-Mode Model}

The dual-mode model hypothesizes differing effects of stress depending on the particulars of the stage of stressor timing and memory phase (de Kloet et al., 1999; Joëls et al., 2006; Schwabe et al., 2012). Specifically, this model proposes that stress generates first a memory formation mode and then a memory storage mode. The rapid autonomic nervous system and non-genomic cortisol hormones induce the memory formation mode, which eases the 
creation of new memories. This mode facilitates the formation of stressor-related memories (i.e., information that encountered during and related to the context of the stressful task) due to an increased excitability in brain areas associated with memory, including the hippocampus, amygdala, and prefrontal cortex. However, this memory formation mode also reduces the ability to retrieve stressor-unrelated information (i.e., non-integral information encountered outside the time or context of encoding). Thus, when acute stress occurs prior to or during retrieval, memory performance will likely be impaired, particularly for information unrelated to the stressor. Later, the slower genomic cortisol initiates a memory storage mode, which facilitates the consolidation of stressor-related material but impairs the formation of stressor-unrelated memories. Additionally, during this stage, memory retrieval continues to impair retrieval processes due to the delayed cortisol hormones.

In essence, the dual-mode model predicts contrasting effects of stress (i.e., enhancing or impairing) on memory performance depending on the memory phase and the convergence of the stressor and the event in time and space. Specifically, if stress is experienced during encoding, effects of stress on memory depend on the time delay between the stressor and the encoding. If stress occurs immediately prior to or during encoding, memory performance (especially for materials related to the stressor) is enhanced, because the memory formation mode is active. Likewise, consolidation is enhanced during the memory storage mode. If there is a long delay (i.e., $30+\mathrm{min}$ ), however, stress impairs encoding because the memory storage mode is active. On the other hand, post-encoding stress enhances memory performance, as encoded information is promoted in both the quick memory formation mode and the slower memory storage mode. Finally, stress experienced prior to or during retrieval impairs memory performance, because both nongenomic and genomic cortisol actions inhibit memory retrieval (Joëls et al., 2006).

\section{Temporal Dynamics Model}

The temporal dynamics model emerged from a contradictory literature in which much of the research concluded that high stress impedes hippocampal functioning, while other findings suggested intact hippocampal memory processing, particularly for emotional memories (see Diamond et al., 2007, for an elaborate discussion). In response to these contradictions, researchers developed a nuanced model, which posits a similar hypothesis as the dual-mode model, by focusing on the specific effects of timing rather than a global interpretation. Whereas the dual-mode model focuses more on the role of the autonomic nervous system and glucocorticoid activity on relevant brain structures, the temporal 
dynamics model specifically examines hippocampal, amygdala, and PFC functioning on a timeline to better explain contrasting results.

According to the temporal dynamics model, two distinct stages of brain area activation occur when acute stress is experienced (Diamond et al., 2007). The effects of stress (i.e., positive or negative) on cognitive functioning can thus differ depending on stage, complementing the predictions from the dual-mode model. Specifically, in the early stage of acute stress, memory-related neuroplasticity (i.e., brain's ability to form and change neural pathways) increases in the hippocampus and amygdala. In contrast, stress immediately suppresses PFC functioning. If stress and learning converge at the same time during this first stage, memory encoding can be enhanced. However, after a short period of time (around $30 \mathrm{~min}$ ), the hippocampus' state of plasticity reverses. A similar reversal occurs in the amygdala, though this alteration takes longer. Thus, if a delay occurs between acute stress and hippocampal-dependent learning, memory formation is impaired. Impairments in the functioning of the PFC also continue, eventually recovering depending on the intensity of the stress experienced.

\section{Executive Control Model}

Another relevant model is the executive control model (Gagnon \& Wagner, 2016), which mainly focuses on the role of PFC in the stress-memory relationship. When acute stress occurs, catecholamines such as adrenaline and dopamine reduce firing in the PFC. In addition to this impairment of PFC functioning, glucocorticoids strengthen the catecholaminergic impairments to executive functioning (Gagnon \& Wagner, 2016). The executive control model posits that because executive functions support memory encoding and retrieval, and because stress disrupts various executive functions (e.g., working memory, cognitive flexibility, etc.), stress impairs memory performance at both encoding and retrieval phases. However, this model also suggests that because stress can shift attention towards stressor-related material, stress experienced prior to or during encoding should facilitate encoding when the information is integral to the stressor - at the cost of information unrelated to the stressor (Gagnon \& Wagner, 2016; Mather \& Sutherland, 2011).

\section{Empirical Support for the Current Models}

These contemporary models are largely complementary and findings from the fundamental 
memory field suggest considerable empirical support for them. For example, some research has specifically examined variations in the interval between stressor and encoding, showing that such variations can alter memory performance (Diamond et al., 2007; Quaedflieg \& Schwabe, 2018; Schwabe et al., 2012). Specifically, in line with the dual-mode model and temporal dynamics model, some studies suggest that when participants experience stress immediately before encoding, their recognition performance is enhanced, but when they experience stress 30 minutes before encoding, their memory performance is impaired (e.g., Quaedflieg et al., 2013; Zoladz et al., 2011). Another study examined acute stress effects at encoding on a longer timescale (Vogel \& Schwabe, 2016a). Participants showed enhanced recognition memory for information encoded during the stressor (associated with the sympathetic stress response), as well as for information encoded 41-65 minutes after the stressor (associated with the cortisol stress response). However, the recognition of information encoded after 60 minutes (i.e., around when genomic cortisol actions begin) was neither enhanced nor impaired. Therefore, perhaps memory enhancements can last slightly longer than the predicted 30-minute window hypothesized by the models, given that both autonomic activation and non-genomic cortisol hormones induce the memory formation mode. Such research also partially supports the hypothesized idea that slower genomic cortisol actions shift the brain to the memory storage mode. However, although these findings reveal a lack of encoding enhancements, they do not show specific memory impairments. The authors suggest that perhaps impairing effects of genomic cortisol on memory may only show after an even longer time period (i.e., longer than two hours) or for more distinct or newer contexts (Vogel \& Schwabe, 2016a). Finally, also in line with these models - and emphasized by the executive control model - are studies showing that encoding enhances recognition and free recall memory for details that are stress-related or central, but not for unrelated peripheral details (e.g., Smeets et al., 2007; Wiemers et al., 2013). In sum, though some research supports the models' hypotheses regarding timingdependent encoding stress effects on memory, the intricacies of specific timing are still not fully understood (Quaedflieg \& Schwabe, 2018).

When examining acute stress effects during retrieval on memory performance, all three models predict memory impairments. Indeed, a considerable amount of research supports this idea, with studies showing recognition and free recall quantity impairments during retrieval after acute stress exposure (e.g., Domes et al., 2004; Kuhlmann et al., 2005a; Smeets, 2011). Most of this work has investigated memory retrieval 30-minutes after stressor exposure, that is, during the memory formation mode (Quaedflieg \& 
Schwabe, 2018). However, one study investigated recognition performance 90 minutes after the experience of acute stress (i.e., during the memory storage mode), and found even greater memory impairments (Schwabe $\&$ Wolf, 2014). In line with the dual-mode model, these findings suggest that both non-genomic and genomic glucocorticoid activity impair retrieval (Schwabe \& Wolf, 2014). Interestingly, this study and another similar study examining free recall quantity found no retrieval impairments for memory tested right after the stressor (Smith et al., 2016). Additionally, some experiments have even showed recall enhancements for stress experienced during retrieval (Hupbach \& Fieman, 2012) and positive correlations between autonomic arousal and immediate memory recall performance (Schönfeld et al., 2014). These findings strengthen the hypothesis that memory impairments are chiefly due to non-genomic and genomic cortisol activity, indicating that retrieval stress experienced before any glucocorticoid actions may not impair memory performance. In sum, these findings generally support the models' predictions of retrieval impairments after acute stress.

\section{Summary of the Current Models}

These contemporary models focus specifically on the timing of the stressor at encoding or retrieval, a feature overlooked in earlier hypotheses (de Kloet et al., 1999; Diamond et al., 2007; Joëls et al., 2006; Schwabe et al., 2012). Specifically, the dual-mode model and temporal dynamics model suggest that the rapid stage of acute stress during encoding can coordinate brain areas such as the hippocampus and amygdala, leading to enhanced memory performance, particularly for stress-related details. In addition, the executive control model posits impairing effects of encoding stress on memory performance, but makes an exception for detail type, suggesting that stress at encoding facilitates stressrelated details (i.e., information directly relevant to the stressor) over unrelated details. This relates to Easterbrook's idea of attention towards central cues over peripheral cues under stress (Easterbrook, 1959; Gagnon \& Wagner, 2016; Mather \& Sutherland, 2011; Shields et al., 2017). The contemporary models also generally predict that stress experienced prior to retrieval will impair memory performance, particularly for tasks requiring PFC involvement (Diamond et al., 2007; Gagnon \& Wagner, 2016; Joëls et al., 2006).

Together, these models provide an initial framework for considering stress in eyewitness contexts. Specifically, the dual-mode model and temporal dynamics model suggest that if an eyewitness experiences stress while encoding a crime, their memory performance- 
particularly for stressor-related details—may be enhanced. In eyewitness contexts, it is likely that the stressor and the encoding occur at the same time, but that is not necessarily always the case. For example, in a blinded kidnapping or hostage situation, victims may be stressed prior to actually seeing the perpetrator or other crime-relevant details. In these situations, where there is a long delay between stress and encoding, victims' memories are more likely to be harmed. The models also suggest that if an eyewitness or victim experiences acute stress before retrieval, their memory performance may be impaired. In this thesis, I will focus on the conceptual foundations from these contemporary models that suggest an intricate interplay between stressor timing and memory performance.

\section{Effects of Acute Stress at Encoding on Memory Overview of Research to Date}

Two main areas of psychological research have investigated how stress at encoding affects memory performance, namely the eyewitness memory field and fundamental memory field. These fields have been interested in the impact of acute stress on both recognition and recall performance. In general, findings reported in the eyewitness memory research field tend to conclude that stress experienced at encoding harms memory recognition (e.g., Deffenbacher et al., 2004; Morgan et al., 2004; Valentine \& Mesout, 2008). For example, Morgan et al. (2004) investigated stress and identification decisions in a study of active-duty military personnel who were participating in a survival school training exercise. Participants experienced one low-stress and one high-stress interrogation, and were later asked to make identification decisions for the two interrogators from a live lineup (Study 1) or a photo lineup (Study 2). In two subsequent studies, participants were in either a high stress (Study 3) or low stress condition (Study 4) and made an identification decision from a sequential photo lineup. Regardless of assessment method, identification performance was worse for high-stress interrogators compared to lowstress interrogators. Eyewitness experiments conducted in the laboratory have also found negative effects of encoding stress on recognition performance. A meta-analysis (Deffenbacher et al., 2004) analyzed 27 independent tests regarding effects of stress on eyewitness identification accuracy and found that acute stress had a negative effect on identification accuracy (average effect size of $h=-.31$ ). More recent laboratory studies reached the same conclusion (e.g., Davis et al., 2019; Pezdek et al., 2020). However, some other recent work from the eyewitness field shows no negative effect of acute stress on identification performance. For example, 123 participants in a study by Sauerland and 
colleagues (2016) witnessed a staged theft after being exposed to a laboratory stressor or a control procedure. One week later, they were asked to identify the thief from targetpresent and target-absent lineups. The results indicated that encoding stress did not have an effect on lineup performance.

Of note, these studies sometimes report slightly different measures of recognition. For example, older studies tended to examine overall proportion of correct responses (e.g., Deffenbacher et al., 2004). More recent experiments often use a broader range of signal detection measures (i.e., hits, false alarms, sensitivity [ $d$ '], response bias [c]; e.g., Davis et al., 2019; Pezdek et al., 2020; Sauerland et al., 2016). Interestingly, some work has suggested that negative effects of encoding stress on memory performance may be driven primarily by an increase in false alarm rates (e.g., Pezdek et al., 2020; Shermohammed et al., 2019). Reporting a wider variety of recognition measures also makes it easier to examine the similarities and differences of results from different experiments. In sum, these recent studies reveal the importance of reporting several recognition measures, such as examining false alarms in addition to hit rates and overall memory performance.

Eyewitness researchers have also investigated the effects of acute stress on recall performance. Field studies have similarly found generally negative effects of encoding stress on free recall ability (Metcalfe et al., 2019). Other studies suggest similar findings when examining cued recall performance, with stressed participants recalling fewer details than those who were not stressed (e.g., Stanny \& Johnson, 2010). Indeed, meta-analytic results of 36 tests regarding effects of stress on recall of crime-related details showed a similar effect for recall accuracy (average effect size of $d=-.31$ ) as identification accuracy, with the authors concluding that they found "considerable support for the hypothesis that high levels of stress negatively impacted both types of eyewitness memory" (Deffenbacher et al., 2004, p.694). However, some eyewitness research suggests inconsistent results; for example, one experiment showed that stressed participants' recalls for an event were less complete but more accurate than the participants who were not stressed (Hulse \& Memon, 2006).

Conversely, fundamental memory research generally suggests that encoding stress can benefit memory performance (e.g., de Kloet et al., 1999; Diamond et al., 2007; Joëls et al., 2006; Schwabe et al., 2012). Experiments specifically examining identification performance are not common in the fundamental research field. However, some fundamental memory studies suggest that stressed participants outperform participants who are not stressed on other types of recognition tasks, including words, pictures, slideshows, photographs, and objects (e.g., Abercrombie et al., 2003; Hoscheidt et al., 
2013; Vogel \& Schwabe, 2016a; Wiemers et al., 2013, 2014). Other experiments, though, show mixed results, such as displaying both recognition enhancements and impairments depending on the valence of the stimuli (Shermohammed et al., 2019), or revealing no effect of cortisone administration at encoding on memory recognition performance (de Quervain et al., 2000).

More often, fundamental memory research indicates that acute encoding stress benefits recall performance. One of the major stress hormones, cortisol, seems to play an important role in this memory facilitation. For example, participants who were administered cortisol outperformed those who were given a placebo on a cued recall task, but the groups showed no statistically significant difference on a recognition task (Buchanan \& Lovallo, 2001). Other fundamental memory studies have found similar results when examining the effects of acute stress on free recall (e.g., Wolf, 2012; Zoladz et al., 2014). For example, one experiment used a laboratory stressor to induce stress in participants before showing them photographs (Wolf 2012, Experiment 2). Findings showed that stressed participants freely recalled more negative photographs than those who were not stressed. Still, some fundamental studies demonstrate conflicting results, such as one study that found that acute stress experienced during encoding impaired free recall as well as recognition memory (Schwabe \& Wolf, 2010).

To summarize, the eyewitness and fundamental memory research fields come to diverging conclusions about the effects of encoding stress on recognition and recall memory performance. With discrepant findings also evident within each field, previous experiments have reported impairing effects, enhancing effects, and null effects of encoding stress on recognition and recall performance. These contrasting findings within and between fields can likely be explained by methodological differences and variations in other relevant factors that may influence the relationship between encoding stress and memory performance.

\section{Methodological Divergence in Research on Encoding Stress and Memory}

The eyewitness and fundamental memory fields have different research goals: while fundamental memory researchers study more basic effects of stress on memory, eyewitness researchers seek to understand the effects of stress on memory in applied forensic contexts. These unique goals lead to different methodological choices. These methodological decisions-regarding stressor type, manipulation checks, retention interval, stressor timing, and stimulus type-likely contribute to the diverging results between fields. 
Some key methodological decisions are summarized in Table 1.1.

When exploring how the type of stressor may affect the stress-memory relationship, it is important to first define what the term 'acute stress' means. In addition to subjective psychological responses (i.e., cognitive and affective reactions), acute stress evokes specific biological reactions (Epel et al., 2018). Researchers in the fundamental memory field use clear terminology to define this biological stress response, taking the time scale of the stress response into account. That is, arousal refers to the activation of the autonomic nervous system, which then releases catecholamines such as adrenaline, whereas acute stress more precisely refers to this rapid response in addition to the slower physiological activation of the HPA axis, prompting the release of cortisol from the adrenal glands. The eyewitness literature tends to use more fluid terminology to describe an eyewitness's response to witnessing a crime (see Bornstein \& Robicheaux, 2009; Dellapaolera, 2019, for discussion), using terms like arousal and stress interchangeably (e.g., Deffenbacher, 1994, vs. Deffenbacher et al., 2004). These differences in terminology may have significant implications for experimental designs and interpretation of results.

Stressor type also frequently differs in experiments across the two fields. The fundamental memory field gives preference to validated stressors such as laboratory-based tasks that have been developed to reliably increase physiological and subjective stress. Two of the most prominent laboratory-based stressors to elicit autonomic and HPA axis arousal and stress include the Cold Pressor Test (CPT; Lovallo, 1975), in which participants immerse their hands in ice water, and the Trier Social Stress Test (TSST; Kirschbaum et al., 1993), in which participants must provide a speech and perform difficult mental arithmetic in front of a panel. A more recently developed task, the Maastricht Acute Stress Test (MAST; Smeets et al., 2012), combines methods from these two tests using alternating blocks of cold-water hand immersion and socially-evaluated mental arithmetic. In contrast to these validated stressors, much of the eyewitness research uses application-focused or scenariorelevant stressors. In the laboratory, these include violent, arousing pictures or videos (Clifford \& Hollin, 1981; Cutler et al., 1987; Kramer et al., 1990; Pezdek et al., 2020), electric shocks (Brigham et al., 1983; Tooley et al., 1987), fake fire alarms (Peters, 1997), threats of injection (e.g., Maass \& Kohnken, 1989; Peters, 1988), and self-reports about trait stress, state stress, or test anxiety (Bailis \& Mueller, 1981; Mueller et al., 1979; Nowicki et al., 1979).

Such stressors are more ecologically valid, better reflecting the experience of stress that may originate from real world experiences. Such scenarios also integrally include the to- 


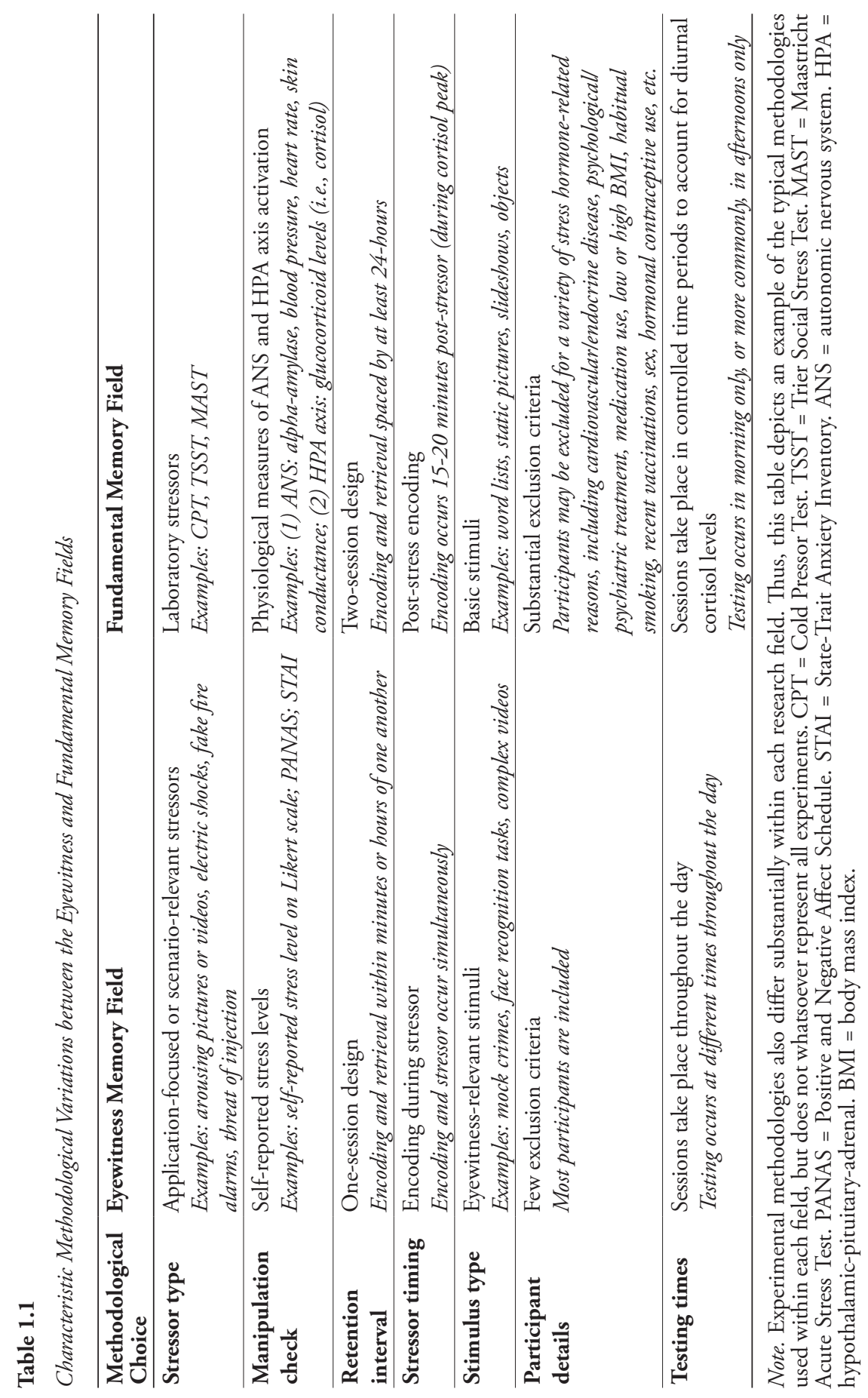


be-remember information, a valuable methodological element that corresponds with an eyewitness experience. Indeed, fundamental theory and research, too, suggest that stressor relevance is an important moderator of the relationship between acute encoding stress and memory performance (e.g., Joëls et al., 2006; Shields et al., 2017). However, it is worth noting that fundamental studies using stressors such as aversive videos produce lower cortisol elevations (i.e., milder stress responses) than other validated laboratory stressors (i.e., TSST, MAST; e.g., Qin et al., 2012), if they produce a cortisol response at all (e.g., Peterson et al., 2014; Wittling \& Pflüger, 1990). Thus, although these eyewitness stressors better reflect reality, they may not sufficiently induce acute stress, making it difficult to draw conclusions regarding the effects of acute stress on memory performance.

In eyewitness field studies, researchers have used a visit to the London Dungeon (Valentine $\&$ Mesout, 2008), interactive police officer training scenarios (Hope et al., 2016; Hulse \& Memon, 2006; Stanny \& Johnson, 2000), or a military survival school program (Morgan et al., 2004) as stressors. These field studies are noteworthy because they are perhaps even more ecologically similar to what an eyewitness in a stressful situation may actually experience. However, they are less controlled than laboratory studies, making it more difficult to isolate the effects of acute stress on memory performance. For example, in Morgan and colleagues (2004), the participants were soldiers engaging in a military survival school program. In addition to undergoing an interrogation that involved threat and actual physical confrontation, soldiers were also food and sleep deprived. The extremely challenging nature of the survival school context, including sleep and food deprivation, does not mirror the experience of a typical eyewitness who unexpectedly experiences a crime during their otherwise normal day-to-day life. That is, these naturalistic elements introduce a variety of additional, and perhaps confounding, factors that could affect the stress-memory relationship beyond effects of acute stress alone. Using such a variety of stress induction methodologies, both between and within research fields, likely induces differing levels of arousal or stress (Bornstein \& Robicheaux, 2009), in addition to exerting differential effects on attention and other cognitive responses. Importantly, differing levels of arousal or stress may result in contrasting effects on memory, with some research suggesting an inverted $U$ relationship as posited by the Yerkes-Dodson law (Yerkes \& Dodson, 1908; e.g., Andreano \& Cahill, 2006; Gagnon \& Wagner, 2016; McCullough et al., 2015; Metcalfe et al., 2019).

Studies in the eyewitness and fundamental memory disciplines also vary in their stress manipulation check. The eyewitness literature often only relies on self-reported stress 
(e.g., Buckhout et al., 1974; Davis et al., 2019; Pezdek et al., 2020). Collecting such reports is important because in reality, self-reports are likely the only information that law enforcement would be able to gather (Bornstein \& Robicheaux, 2009). However, selfreport measures alone cannot verify the effectiveness of a stressor, and, more importantly, they do not always correspond with physiological stress responses (Hellhammer \& Schubert, 2012). Researchers in the fundamental memory field typically use physiological measurements (e.g., heart rate, blood pressure, alpha-amylase, cortisol levels) to confirm that stress was successfully induced and to examine the degree of arousal and/or acute stress (see Shields, 2020, for recommendations). For making claims about acute stress effects on memory performance, confirming both autonomic arousal (i.e., increases in heart rate, blood pressure, alpha-amylase, sweating) and HPA axis arousal (i.e., increases in cortisol) is imperative, particularly for stressors that have not been validated. These manipulation checks ensure validity, confirming that when researchers claim to be examining acute stress, they actually are doing so.

Another factor that differs between the two fields concerns the retention interval. The physiological effects of acute stress in the brain are long-lasting, with genomic cortisol remaining elevated for hours after a stressful event has taken place (Joëls \& Baram, 2009; Joëls et al., 2011; Quaedflieg \& Schwabe, 2018). Many studies in the eyewitness field use single session designs, with encoding and retrieval taking place on the same day - mostly within minutes of each other (e.g., Davis et al., 2019; Hulse \& Memon, 2006; Pezdek et al., 2020; Valentine \& Mesout, 2008). Examining acute stress effects on memory in a short retention interval may be useful for certain applied settings, such as assessing stress effects on statements gathered from witnesses shortly after a stressful experience (e.g., Krix et al., 2016). However, because stress shows opposite effects for consolidation and retrieval phases (e.g., Shields et al., 2017; Wolf, 2017), this methodological decision is problematic for isolating the effects of encoding stress on memory (see Sauerland et al., 2016; Thomas \& Karanian, 2019). Fundamental memory models suggest that acute stress first shifts the brain into a memory formation mode, followed by a memory storage mode (de Kloet et al., 1999; Diamond et al., 2007; Joëls et al., 2006; Schwabe et al., 2012). Memory retrieval is impaired in both of these memory formation and memory storage modes. Thus, if retrieval occurs within minutes or hours of encoding, the memory phases are muddled. This makes it impossible to distinguish from where the effects of stress originate and likely affects study results, as the stress effects act in opposition. Most fundamental memory research overcomes this complication by implementing 
two-session experiments, in which participants return to the lab for the memory test at least 24-hours after the initial stress and encoding session (e.g., Quaedflieg et al., 2013; Shermohammed et al., 2019; Vogel \& Schwabe, 2016a; Wolf, 2012; Zoladz et al., 2014). A longer retention interval may also better reflect the majority of real-world eyewitness experiences, because police interviews and identification procedures rarely occur on the same day as the experienced crime.

In addition to the retention interval, more subtle differences in stressor timing may help explain the discrepant results of previous studies. Whereas most studies discussed here claim to examine stress at encoding, differences in stressor timing could have large ramifications for memory performance (see Quaedflieg \& Schwabe, 2018). For example, many eyewitness studies are primarily interested in memory for events experienced during a stressor, as this scenario relates most closely to the experience of an individual witnessing a crime. Thus, to replicate the applied context, much of the research implements the stressor at the same time as the to-be-remembered stimulus or event (e.g., most experiments in Deffenbacher et al., 2004; Hulse \& Memon, 2006; Morgan et al., 2004). On the other hand, fundamental memory research tends to focus on encoding at cortisol peak, which does not occur until about 15 -minutes post-stressor. Therefore, participants are often stressed before encoding, while encoding occurs during the subsequent cortisol peak (e.g., Wolf, 2012; Zoladz et al., 2014). Studies that investigated the subtleties of stress prior to encoding generally suggest that stress immediately before or during encoding enhances memory recognition and recall performance, whereas stress induced before (i.e., $25 \mathrm{~min}$ or more) encoding typically impairs memory performance (Joëls et al., 2011; Schwabe et al., 2012; Zoladz et al., 2011, 2018). One study examined acute stress effects at encoding on a longer timescale (Vogel \& Schwabe, 2016a). Participants showed enhanced recognition memory for information encoded during the stressor (associated with the sympathetic stress response), as well as for information encoded 41-65 minutes after the stressor (associated with the cortisol stress response). However, other fundamental memory work has suggested that learning under stress can impair free recall and recognition performance (Schwabe \& Wolf, 2010). Speaking to the reallife application, a time delay between stress and encoding could occur. For example, in a blinded kidnapping or hostage situation, victims may be stressed prior to actually seeing the perpetrator or other crime-relevant details. However, for most eyewitness situations, the stressor and encoding occur at the same time, which means that studies using this scenario likely also capture broader effects of the scenario on cognition (e.g., attention; Wulff \& Thomas, 2021). Given the delicate timeline of stress effects on the 
brain (Quaedflieg \& Schwabe, 2018), researchers' decisions regarding stressor timing likely plays a vital role in the inconsistent findings reported in the literature.

Finally, variations in stimuli could also contribute to the inconsistent findings between the eyewitness and fundamental memory fields. Eyewitness researchers often use materials that would be applicable to a forensic scenario, including showing participants criminal events or perpetrator faces (e.g., Davis et al., 2019; Deffenbacher et al., 2004; Morgan et al., 2004; Sauerland et al., 2016). Fundamental memory researchers tend to use less complex stimuli, such as words, static pictures, or slideshows (e.g., Domes et al., 2002; Henckens et al., 2009; Payne et al., 2006; Schwabe et al., 2008a). Such differences in stimuli may play a role in the contrasting findings, as faces are processed and learned differently than other stimuli (e.g., face-specificity hypothesis; Kanwisher \& Yovel, 2006) and humans are generally experts in face recognition (e.g., Diamond \& Carey, 1986). In addition, meta-analytic results from the eyewitness memory field indicate that studies using a staged crime, particularly a live or interactive staged crime, show larger impairments in identification and recall performance (Deffenbacher et al., 2004) than other scenarios (e.g., threat of injection). Thus, the use of differing stimuli or to-beremembered events could contribute to the mixed results between research fields.

\section{Other Relevant Factors in Research on Encoding Stress and Memory}

Other methodological factors may influence effects of encoding stress on memory performance, but do not differ overly between research fields, such as the valence of the stimuli and the type of to-be-remembered detail. Some evidence suggests that emotional valence plays a role in the relationship between encoding stress and memory (Wolf, 2008). In general, many studies suggest that encoding stress effects are larger for emotional materials than neutral materials throughout memory phases (e.g., Buchanan \& Lovallo, 2001; Goldfarb et al., 2019; Kuhlmann \& Wolf, 2006; Wiemers et al., 2019; Wolf, 2012; Zoladz et al., 2011). However, other studies reported mixed or contrasting results (Abercrombie et al., 2003) or showed impaired memory recognition for negative stimuli but enhanced memory recognition for neutral stimuli (Shermohammed et al., 2019). Indeed, one recent meta-analysis found that valence did not moderate the relationship between encoding stress and memory performance, revealing that valence effects regarding encoding stress are not always found (Shields et al., 2017).

Type of detail (i.e., central vs. peripheral) may also differentially affect the stress-memory relationship (Burke et al., 1992; Christianson \& Loftus, 1987; Christianson et al., 1991; 
Echterhoff \& Wolf, 2012). Specifically, the cue utilization hypothesis suggests that stress at encoding may enhance memory for central details, but reduce memory for peripheral details (Christianson, 1992; Easterbrook, 1959). In addition, researchers have examined relevance, with results suggesting that only information relevant to the stressor will be enhanced by stress at encoding (Shields et al., 2017; Smeets et al., 2007; Wiemers et al., 2013). Thus, memory enhancements for stress experienced at encoding may only occur for central, stressor-relevant details.

To summarize, the mixed findings regarding effects of encoding stress on memory performance are likely due to methodological differences and deviations in factors such as valence and detail type. Some methodological variance between eyewitness and fundamental research lines stems from diverse research motives. This thesis sought to bring together these lines of research, combining robust methodology with factors of interest to eyewitness memory, such as investigating pertinent stressor timing and using eyewitness-relevant stimuli (e.g., faces).

\section{Effects of Acute Stress at Retrieval on Memory}

\section{An Overview of Research to Date}

Frequently in the legal system, victims and witnesses are asked to remember the crime and share their story - whether to police or in the courtroom. Both of these situations are unfamiliar to many people and are likely anxiety provoking (Bornstein et al., 2013; Memon et al., 2011; Tinsley, 2001). The stress that arises in these situations may have the potential to influence the statements given by the witnesses. Thus, examining the effects of acute stress experienced during retrieval on recall and rqecognition performance is also important for eyewitness contexts.

Findings from past fundamental research examining stress during retrieval are more consistent than those on the effects of encoding stress. Research generally suggests that experiencing stress prior to or during retrieval has a negative impact on memory performance (Het et al., 2005; Kuhlmann et al., 2005a; Lupien et al., 2009; Wolf, 2009). For example, one recent meta-analysis examined results from 26 published papers investigating retrieval stress effects on memory (Shields et al., 2017). Across studies and paradigms, they found that acute stress impaired retrieval (Hedges' $g=-.22$ ). Additionally, memory researchers often clearly express these established impairing effects of stress on memory retrieval in published papers, with title sentences such as, "Stress impairs memory retrieval," (Wolf, 2017, p.40) and "More than a decade of research has supported a robust 
consensus: Acute stress impairs memory retrieval" (Smith, Floerke, \& Thomas, 2016, p.1046). Yet, little work has focused on investigating retrieval stress on memory from a perspective applicable to eyewitness scenarios, apart from two unpublished doctoral dissertations which generally show that acute stress did not affect lineup accuracy or face recognition performance (Dellapaolera, 2019; Robicheaux, 2016).

\section{Relevant Factors in Research on Retrieval Stress and Memory}

Three main factors suggested by fundamental memory studies are important to consider when studying the relationship between stress experienced at retrieval and memory performance: stressor timing, memory test type, and stimulus valence and type. First, retrieval impairments are strong during the cortisol peak (i.e., 15-20 minutes poststressor) and continue as genomic glucocorticoid effects develop (i.e., around 60-90 minutes post-stressor; de Quervain et al., 1998; Kuhlmann et al., 2005b; Schwabe \& Wolf, 2014; Wolf, 2017). With little or no delay between the stressor and testing, some findings show no effects or even enhancements on recognition and recall quantity memory (e.g., Hupbach \& Fieman, 2012; Schwabe \& Wolf, 2014; Smith et al., 2016). Another experiment revealed that increases in autonomic arousal were positively correlated with immediate memory recall under stress (e.g., Schönfeld et al., 2014), suggesting that perhaps autonomic arousal can actually enhance memory performance when retrieval occurs straightaway. However, little specific research has examined this time period; rather, most studies have focused on examining memory around the cortisol peak or later and not during the stressor. Understanding how stress during retrieval affects memory is important for eyewitness contexts, where the primary interest is how stress that an eyewitness feels during an identification procedure or interview may affect their performance.

Second, the effect of stress during retrieval may differ as a function of memory test. Retrieval stress impairs performance on both recall quantity (e.g., Kuhlmann et al., 2005b; Smeets et al., 2008) and recognition tasks (Domes et al., 2004; Li et al., 2013; Schwabe \& Wolf, 2014). However, evidence suggests stronger negative effects for recall than recognition (see Gagnon \& Wagner, 2016; Wolf, 2017). For example, some experiments using cortisone administration found that cortisol impaired free recall memory but not recognition memory (e.g., de Quervain et al., 2000, 2003). Gagnon and Wagner (2016) suggested that this might be the case because recognition memory relies on familiarity, whereas free recall requires recollection. Recall is associated with greater 
hippocampal dependency (Gagnon \& Wagner, 2016). As a result, acute stress, which directly affects the hippocampus, may more strongly impair free recall, while sparing familiarity-based judgments. Thus, the type of memory test may influence stress effects on memory performance.

Third, retrieval stress impairments seem to be larger for negative than for neutral information (e.g., Gagnon \& Wagner, 2016; Kuhlmann et al., 2005a, 2005b; Schönfeld et al., 2014). For example, a meta-analysis found that valence moderated the relationship between retrieval stress and memory, such that acute stress impaired retrieval to a greater extent for negative (Hedges' $g=-.30$ ) and positive information (Hedges' $g=-.39$ ) than for neutral information (Hedges' $g=-.14$; Shields et al., 2017). One explanation for this effect is that emotional information is more salient, better capturing attention and thus becomes a higher priority than neutral information (Bannerman et al., 2010; Mather $\&$ Sutherland, 2011). Indeed, the amygdala, often described as the brain's center for emotions, is especially activated during emotional stress, and exerts influence on other memoryassociated brain areas such as the hippocampus (e.g., McGaugh, 2004; Roozendaal et al., 2009). Besides stimulus valence, stimulus type is also an important factor to consider in eyewitness contexts. Limited work on retrieval stress has investigated eyewitness-relevant stimuli such as faces (but see $\mathrm{Li}$ et al., 2013, 2014). These studies have revealed mixed findings, with one experiment suggesting impairments for face recognition sensitivity (i.e., $d$ '; Li et al., 2013) and another suggesting no effect on face recognition accuracy (Li et al., 2014). Differences could exist regarding the retrieval of faces compared with other forms of stimuli. For example, some research suggests that faces are processed and learned differently than other stimuli (e.g., face-specificity hypothesis; Kanwisher \& Yovel, 2006), and other research suggesting that humans are generally experts in face recognition (e.g., Diamond \& Carey, 1986). As such, examining overlooked forms of eyewitness-related stimuli like faces in the context of the effects of retrieval stress on recognition memory is necessary.

To summarize, the evidence base to date suggests that stress during retrieval impairs memory performance. Certain factors may influence these negative effects, including stressor timing, memory test type, and stimulus valence and type. However, acute stress effects on memory during retrieval have not been investigated in an eyewitness or forensic context. This thesis aimed to further examine retrieval stress effects using eyewitnessrelevant methodology. 


\section{Mitigating Negative Effects of Acute Retrieval Stress}

Experiencing retrieval stress can result in memory impairment (e.g., Wolf, 2017), and therefore finding ways to mitigate negative effects of acute stress at retrieval would be valuable in many circumstances: for students facing examinations, for those who work in inherently stressful occupations (e.g., surgeons, pilots, police officers, etc.), and for eyewitnesses or victims of crimes. Some recent work has begun to examine potential ways to reduce the negative effects of stress on memory performance. For example, one study examining retrieval practice found that engaging in retrieval practice (i.e., taking practice tests) rather than simply restudying the materials mitigated the negative effects of retrieval stress (Smith et al., 2016). Teaching people coping mechanisms for dealing with stress could also help reduce negative effects of stress on retrieval performance (Vogel \& Schwabe, 2016b), whether by focusing on changing the stressor directly or by regulating negative emotions associated with the stressor. Indeed, research in academic settings shows that using such coping mechanisms can be effective against stress (Pincus \& Friedman, 2004; Zeidner, 1995). However, these interventions may not be effective in eyewitness contexts, where time and resources are limited and the stakes are higher.

Even when police are sensitive and do not overtly impose stress on interviewees, eyewitnesses may still find the process of a police interview stressful. Being interviewed by the police is an unfamiliar situation for most people, and being uncertain about how the interview will transpire (Sydeman et al., 1997) or suffering from social anxiety (particularly when dealing with authority figures, e.g., Leitenberg, 1990) may also increase eyewitness stress. Indeed, research examining other types of interview settings suggests heightened stress levels for interviewees (e.g., McCarthy \& Goffin, 2004; Posthuma et al., 2002). Because stress cannot always be avoided in eyewitness situations, one tactic for managing stress could be to use an easily learnable form of cognitive reappraisal to reframe the way one views stress. For example, one such intervention, called arousal reappraisal, has been used to promote cognitive performance in stressful situations by shifting individuals' experience of stress from a threat state to a challenge state by guiding them to embrace the stress they are feeling as adaptive and useful rather than harmful (e.g., Jamieson et al., 2018; Mendes \& Park, 2014). Recent research showed that the use of such an arousal reappraisal technique "fostered an overall adaptive and flexible pattern of cardiovascular, neuroendocrine, and psychological responses" for stressed participants (Jentsch \& Wolf, 2020, p. 7). However, no research to date has directly investigated the use of such an intervention to mitigate negative effects of acute stress on (eyewitness) memory performance. 
In sum, substantial research suggests impairing effects of retrieval on memory performance, but few studies have investigated ways to mitigate those negative effects. Further, research on such interventions that could be applied in eyewitness settings, such as during police interviews, is very limited. Thus, the final portion of this project turned towards testing a potential applied intervention. Specifically, this thesis aimed examine the efficacy of an arousal reappraisal intervention in reducing the impairing effects of stress on both recognition and free recall memory performance.

\section{Outline of the Current Thesis}

In this chapter, I have highlighted three main gaps in the eyewitness stress-memory literature: a lack of 1) current knowledge about experts' and layperson's beliefs regarding stress effects on memory performance, 2) experiments using contemporary, rigorous methodology to investigate effects of encoding and retrieval stress on the recognition of eyewitness-relevant stimuli, and 3) studies examining ways to reduce negative effects of retrieval stress on recognition and free recall performance. This thesis aims to address these gaps through one survey and three empirical experiments. Each experiment in this thesis was pre-registered and Experiment 4 was accepted as a registered report.

In Chapter 2 (Experiment 1), I will present findings from the first in-depth survey regarding current beliefs about stress effects on memory from memory experts and laypeople. Specifically, I surveyed eyewitness memory experts, fundamental memory experts, and laypeople regarding their beliefs on a number of statements related to stress effects on (eyewitness) memory performance. This exploratory study reported proportions of each group endorsing or rejecting each statement. Additionally, I examined differences between the groups, comparing eyewitness memory experts with fundamental memory experts, and comparing experts as a whole with laypeople. I found that large proportions of experts in both research fields agreed that very high levels of stress impair the accuracy of eyewitness testimony. Conversely, a majority of fundamental memory experts, but not eyewitness memory experts, endorsed the idea that stress experienced during encoding can enhance memory performance, mirroring the diverging experimental results from these two research fields. Additionally, a majority of layperson respondents endorsed some mistaken commonsense beliefs, such as incorrectly thinking that police officers' memories are less affected by stress than other witnesses (e.g., Stanny \& Johnson, 2000) and falsely believing that stress can cause repressed memories (e.g., Otgaar et al., 2019).

As presented in Chapter 3 (Experiments 2 and 3), I next examined the effects of acute 
stress during encoding and retrieval on face recognition memory. I incorporated rigorous methodology by using a standardized laboratory stressor, assessing the stress induction with subjective and physiological measures, employing a 24-hour retention interval to distinguish between memory phases, and examining stress both during and prior to encoding and retrieval. As such, the experimental studies aligned with recommended guidelines about designing and interpreting studies examining stress and cognition (Shields, 2020). Drawing on the dual-mode model and temporal dynamics model and related empirical findings, I predicted enhancing effects of stress at encoding and impairing effects of stress at retrieval on recognition performance. Specifically, in Experiment 2, participants were stressed before encoding faces, before the recognition test, before encoding and retrieval, or neither time. However, despite stress-induced increases in physiological (i.e., blood pressure) and subjective (i.e., negative affect) measures, neither encoding stress nor retrieval stress affected recognition performance. In light of these findings, a followup experiment investigated whether stress experienced at encoding affected memory differently for different stimuli, a possible explanation for these unanticipated results. Thus, in Experiment 3, I examined the effects of encoding stress (i.e., stressed vs. not stressed) on recognition performance and added a within-subjects factor of stimulus type by testing memory for both faces and words. I predicted that words would show the predicted enhancing effects of encoding stress, while faces would not be affected by stress at encoding. Again, the stress manipulations were successful, with participants in the stress condition showing increased negative affect, blood pressure, and cortisol levels than those in the control conditions. Still, results showed no statistically significant effects of acute stress on either stimulus type. The findings from these two experiments suggest that acute stress does not affect face recognition memory performance, nor word recognition performance for encoding stress.

In Experiment 4, I applied previous work regarding a cognitive intervention to the established findings concerning the impairing effects of stress during retrieval on recognition and free recall memory (Chapter 4). Specifically, I tested whether an arousal reappraisal intervention, which has been successful at improving cognition in other settings (e.g., Jamieson et al., 2018), could help alleviate the negative effects of stress on memory performance. Participants viewed four detailed scenes and returned to the lab the next day. Before the stress induction or control task, participants were given a placebo or intervention task. The intervention instructed participants to view their stress and subsequent bodily responses as an adaptive response that would enhance their task 
performance, whereas the placebo instructed participants to ignore their stress. After being stressed or engaging in the control task, participants completed a memory test, including both recognition and free recall elements. I predicted that stressed participants who received the placebo task would perform worse on the memory tasks than nonstressed participants who received the placebo task. However, I also predicted that stressed participants who received the intervention task would outperform stressed participants who received the placebo task, thus mitigating the negative effects of stress on memory performance. As expected, participants showed increased negative affect and blood pressure compared to non-stressed participants. However, the memory results showed no support for either hypothesis. I found no retrieval stress effects on memory performance, and no effects of the arousal reappraisal on memory performance, nor on measures of affect, task demands, or coping resources. Thus, Experiment 4 broadly suggests an absence of a reliable effect of acute retrieval stress or arousal reappraisal on recall and recognition memory performance.

In the General Discussion (Chapter 5), I provide an overview of the key findings, consider theoretical as well as practical implications of the results from this thesis, discuss limitations of the current studies, and suggest recommendations and avenues for future research regarding the effects of acute stress on eyewitness memory performance. 


\section{CHAPTER 2}

\section{Memory Experts' and Laypeople's Beliefs}

about the Effects of Acute Stress on (Eyewitness) Memory Performance \& Cognition, 49(3), 401-421. https://www.doi.org/10.3758/s13421-020-01115-4 


\begin{abstract}
This survey examined lay and expert beliefs about statements concerning stress effects on (eyewitness) memory. Thirty-seven eyewitness memory experts, 36 fundamental memory experts, and 109 laypeople endorsed, opposed, or selected don't know responses for a range of statements relating to the effects of stress at encoding and retrieval. I examined proportions in each group and differences between groups (eyewitness memory experts vs. fundamental memory experts; experts vs. laypeople) for endorsements (agree vs. disagree) and selections (don't know vs. agreeldisagree). High proportions of experts from both research fields agreed that very high levels of stress impair the accuracy of eyewitness testimony. A majority of fundamental experts, but not eyewitness experts, endorsed the idea that stress experienced during encoding can enhance memory. Responses to statements regarding moderating factors such as stressor severity and detail type provided further insight into this discrepancy. Eyewitness memory experts more frequently selected the don't know option for neuroscientific statements regarding stress effects on memory than fundamental memory experts, although don't know selections were substantial among both expert groups. Laypeople's responses to eight of the statements differed statistically from expert answers on topics such as memory in children, in professionals such as police officers, for faces and short crimes, and the existence of repression, providing insight into possible 'commonsense' beliefs on stress effects on memory. These findings capture the current state of knowledge about stress effects on memory as reflected by sample of experts and laypeople, and highlight areas where further research and consensus would be valuable.
\end{abstract}




\section{Introduction}

Witnesses often experience acute stress in forensic contexts, whether during a crime or during subsequent police interviews (Bornstein et al., 2013; Davis, 2016; Yuille

\& Cutshall, 1986). Facing difficult, frightening, and emotional events can trigger a subjective and physiological stress response from the witness (Bornstein \& Robicheuax, 2009). A body of research has been devoted to examining the potential effects of acute stress on memory, but results have been inconsistent. Equally important, there is little information about current memory experts' and laypeople's knowledge about the stressmemory relationship. Memory experts in different research domains, such as eyewitness memory experts and fundamental memory experts, as well as laypeople, may have different understandings of this relationship. If different perspectives exist, such differences could emerge in courtroom settings in problematic ways. For example, memory researchers from different fields might be asked to be expert witnesses in court, and then provide diverging statements concerning stress-memory relationships. Additionally, laypeople acting as jurors may evaluate eyewitness evidence based on their pre-existing 'commonsense' beliefs. To capture the contemporary perspectives of memory experts and laypeople, I examined current memory experts' and laypeople's beliefs about the effects of acute stress on memory by means of a targeted survey.

\section{Stress and Memory: An Ongoing Discussion}

Two groups of memory researchers have examined the effects of acute stress on memory encoding and retrieval. One group predominantly concentrates on memory in applied settings, such as eyewitness memory, and the other group mainly focuses on fundamental memory research, including neurobiological research related to basic memory processes (e.g., memory performance for non-complex stimuli, such as word lists or numeric strings). Generally, across fields, research shows that acute stress at retrieval impairs memory (e.g., Schwabe et al., 2012; Shields et al., 2017; Wolf, 2017), although limited research on this specific issue has been conducted in the eyewitness memory context (see Dellapaolera, 2019; Robicheaux, 2016). However, findings concerning the effects of acute stress at encoding on memory performance appear to be discrepant between research fields (Christianson, 1992; Schwabe et al., 2012; Shields et al., 2017). Eyewitness memory research mostly concludes that encoding stress impairs eyewitness memory. For example, a meta-analysis of 27 eyewitness memory studies suggested that heightened stress exerts a negative effect on eyewitness memory for both the perpetrator and details 
associated with the crime (Deffenbacher et al., 2004). Eyewitness memory researchers often cite such research as evidence that the negative effect of encoding stress on memory is a conclusive finding (e.g., Schmechel et al., 2006; Yarmey \& Jones, 1983). For example, Schmechel and colleagues (2006) stated that "highly stressful situations may make an experience seem especially vivid, but such stressors can reduce the ability to recall details about a person's face", declaring this summary of stress effects as an "empirical answer" (p. 179). Field studies in this area have also highlighted a negative effect of severe encoding stress on memory (e.g., Metcalfe et al., 2019; Stanny \& Johnson, 2000; Valentine \& Mesout, 2008). For instance, in one study, active-duty military personnel participated in a survival school training exercise (Morgan et al., 2004). During training, participants experienced one low-stress interrogation and one high-stress interrogation and were later asked either to make identification decisions for each of the two interrogations from a live lineup (Study 1) or a photo lineup (Study 2). In two subsequent studies, all participants were either in the high stress (Study 3) or low stress condition (Study 4) and made an identification decision from a sequential photo lineup. Regardless of assessment method, identification performance was better for low-stress interrogators compared to high-stress interrogators. However, it should be noted that several other factors in this field study are potentially confounding variables, such as the fact that all soldiers participating in the research were deprived of food and sleep for 48 hours prior to the interrogations. These naturalistic elements of the survival training context likely impacted the stress-memory relationship beyond the effects of acute stress alone.

The view that stress at encoding negatively affects subsequent memory is in contrast to findings reported in fundamental memory research, which demonstrate that acute stress at encoding can actually enhance memory performance (e.g., Henckens et al., 2009; Shields et al., 2017; Vogel \& Schwabe, 2016a; Wolf, 2012). These findings can be accounted for in terms of the cognitive effects of physiological stress responses triggered by acute stress. When we experience acute stress, adrenaline and noradrenaline are quickly released, followed by the slower release of cortisol from the activation of the HPA axis (e.g., Joëls \& Baram, 2009, Joëls et al., 2011; Robbins, 1984; Ulrich-Lai \& Herman, 2009). The rapid catecholaminergic and non-genomic glucocorticoid actions set the brain in a memory formation mode (Diamond et al., 2007; Joëls et al., 2006). If encoding occurs during this part of the memory phase, acute stress should enhance memory formation for stress-related material, while also impairing retrieval of material unrelated to the stressor (e.g., Diamond et al., 2007; Joëls et al., 2006; Quaedflieg \& Schwabe, 2018; Shields et al., 2017). Methodological differences between eyewitness research and fundamental 
memory research may explain these contradictory results. For example, differences in the type and severity of stressors, the timing between a stressor and encoding, and retention intervals between encoding and retrieval could result in varied findings (for discussions of potential participant and study design moderators, see Sauerland et al., 2016; Shields et al., 2017; Shields, 2020; Thomas \& Karanian, 2019).

These diverging research findings with respect to how stress at encoding impacts memory performance suggest that disagreement might also exist between different types of experts about topics related to acute stress and memory. Additionally, beliefs held by the general population do not always mirror expert knowledge (e.g., Benton et al., 2006; Simons \& Chabris, 2011; Yarmey \& Jones, 1983), likely because laypeople have not learned about or studied these topics in the way that experts have. As such, lay beliefs tend to be more 'commonsense', based on gut feelings or speculations rather than on scientific findings. Past surveys have examined some general beliefs about stress and memory among both lay and expert samples. Table 2.1 presents an overview of 17 published surveys, published from 1979, with the most recent published in 2010. Across all 17 surveys, $79 \%$ of laypeople agreed that high stress harms the accuracy of eyewitness testimony (survey responses ranging from $41 \%$ to $92 \%$ ). Three surveys examining experts' beliefs about the negative effects of stress on eyewitness memory between 1983 and 2001 show a slight decline in agreement (Kassin et al., 1989, 2001; Yarmey \& Jones, 1983). In 1983, 88\% of experts $(N=16)$ agreed with the statement that When a person experienced extreme stress as the victim of a crime, helshe will have reduced ability to notice and remember the details of the event (Yarmey \& Jones, 1983). In 1989, 73\% of eyewitness experts ( $N=63)$ agreed that the statement Very high levels of stress impair the accuracy of eyewitness testimony was reliable enough to present in court (Kassin et al., 1989). By 2001, agreement levels had dropped to $60 \%$ ( $N=62$; Kassin et al., 2001). Similarly, $79 \%$ of experts agreed that the evidence supported that statement in 1989, whereas 11 years later $65 \%$ of experts agreed that high levels of stress impaired the accuracy of eyewitness testimony. These surveys among experts suggest that consensus concerning the stress-memory relationship has been declining over the years. However, the statement used in previous surveys does not include an indication of memory phase (i.e., encoding or retrieval). Additionally, the most recent survey investigating expert opinions on this relationship is nearly two decades old (Kassin et al., 2001), and many studies regarding stress and memory have been published since then. For example, all 90 papers included in the Shields et al. (2017) meta-analysis on this topic were published in or after 2001, highlighting the need for a more contemporary assessment of opinion. 
Other surveys focused on beliefs about emotional events. For example, in one survey $80 \%$ of layperson respondents endorsed the notion that emotional events are usually remembered more accurately than memories for everyday events (Conway et al., 2014). More recently, 54\% of a surveyed lay sample agreed or strongly agreed that experiences involving very strong emotions and memories of emotionally negative experiences were more accurately remembered than emotionally moderate or weak, neutral, or positive experiences (Akhtar et al., 2018). The finding that most laypeople believe that emotional intensity gives rise to accurate memories seems to be out of line with other surveys indicating that laypeople generally believe acute stress harms eyewitness memory. However, although emotional intensity and stress often relate to similar applied matters, the two cannot be fully equated. For example, eyewitness scenarios often involve both negative emotionality and stress (e.g., witnessing an unexpected fatal car accident or lifethreatening assault). Other experiences, however, may be emotionally negative, but not necessarily elicit an acute stress response (e.g., a failed relationship or the death of an ill parent). The relationship between emotional intensity and memory accuracy has also been investigated in one expert sample (Akhtar et al., 2018). Forty-six percent of experts agreed or strongly agreed with the idea that emotional experiences were more accurately remembered than neutral or positive experiences. However, 54\% of experts disagreed with this statement suggesting a similar lack of consensus between experts regarding topics associated with the effects of acute stress on memory.

Past surveys investigating stress and memory have typically included a single statement concerning the effects of acute stress on memory (i.e., Very high levels of stress impair the accuracy of eyewitness testimony). However, the complexity of the effects of acute stress on memory cannot be meaningfully captured in this single item. A more indepth investigation of laypeople's and experts' understandings about effects of stress on memory is valuable for two reasons. First, the complexity of this particular topic is evident through the numerous moderators about which beliefs have not yet been examined. Specifically, previous surveys have not included questions about specificity of stressor timing (i.e., encoding vs. retrieval; see Joëls et al., 2011; Quaedflieg \& Schwabe, 2018), the neuroscientific theories behind stress effects on memory, and the potential moderators of the acute stress-memory relationship (i.e., age, type of memory test, stress severity, detail type, etc.). Understanding expert beliefs about these moderators will also elucidate which factors require further investigation, setting important directions for future research on this topic. Second, understandings about stress and memory can have real life consequences. Laypeople's views may enter the legal decision-making process 
Table 2.1

Percentage of Layperson Respondents Agreeing With the Statement on the Negative Effects of High Stress on Eyewitness Memory in Past Surveys

\begin{tabular}{llllc}
\hline Authors & Year & Country & Sample & $\begin{array}{c}\% \\
\text { endorsed }\end{array}$ \\
\hline Loftus & 1979 & USA & 500 students & 67 \\
\hline Yarmey \& Jones & 1983 & Canada & 60 students and 60 local adults 57 \\
\hline Deffenbacher \& Loftus & 1982 & USA & 76 students & 85 \\
\hline Deffenbacher \& Loftus & 1982 & USA & 100 students & 79 \\
\hline Deffenbacher \& Loftus & 1982 & USA & 46 jurors & 41 \\
\hline Deffenbacher \& Loftus & 1982 & USA & 43 jurors & 53 \\
\hline Noon \& Hollin & 1987 & UK & 28 students & 79 \\
\hline Noon \& Hollin & 1987 & UK & 24 law students & 79 \\
\hline Noon \& Hollin & 1987 & UK & 24 potential jurors & 67 \\
\hline Kassin \& Barndollar & 1992 & USA & 39 students and 40 local adults & 82 \\
\hline Schmechel, O’Toole, Easterly, \& Loftus* & 2004 & USA & 1,007 potential jurors & 80 \\
\hline Benton, Ross, Bradshaw, Thomas, \& Bradshaw & 2006 & USA & 111 jurors & 68 \\
\hline Read \& Desmarais & 2009 & Canada & 201 potential jurors & 79 \\
\hline Read \& Desmarais & 2009 & Canada & 200 potential jurors & 92 \\
\hline Read \& Desmarais & 2009 & Canada & 598 potential jurors & 88 \\
\hline Magnussen, Melinder, Stridbeck, \& Raja & 2010 & Norway & 164 members of juror pool & 79 \\
\hline Magnussen, Melinder, Stridbeck, \& Raja & 2010 & Norway & 1,000 potential jurors & 84 \\
\hline
\end{tabular}

Note. $\%$ endorsed $=$ percentage of participants who believed in negative effects of high stress on eyewitness memory. Potential jurors $=$ general public. ${ }^{*}=$ Statement in this survey was "if an eyewitness was under high stress at the time of the crime, the eyewitness will have better recall for the details of the event", percentage in table represents those who believed this statement was false.

when they act as jurors, and research suggests their beliefs can impact their decisions about credibility and guilt. For example, mock jurors in one study expressed whether they believed arousal helps or hinders memory performance before reading a trial transcript of a crime involving an eyewitness experiencing high arousal (Bornstein et al., 2008). Ninety-eight percent of participants who believed that arousal helps memory ( $N=44)$ selected a guilty verdict, whereas only $44 \%$ of participants who believed that arousal hinders memory $(N=25)$ selected guilty. Thus, commonsense beliefs about the effects of arousal or stress on memory may also influence courtroom decisions in real 
life. Furthermore, experts' opinions can also affect legal decision-making when they testify as expert witnesses. Indeed, the effects of stress on the accuracy of eyewitness testimony was identified as the topic second most frequently testified about by experts across 21 eyewitness-related topics (Kassin et al., 1989). Therefore, even though the different research fields focusing on stress and memory do not show conclusive findings, understanding laypeople's and expert's beliefs with respect to this topic is still vital due to these potential real-world consequences.

\section{The Current Survey}

The current survey assessed laypeople's and experts' beliefs about the relationship between acute stress and memory. The survey items used are presented in Table 2.2. One group of lay respondents and two groups of expert respondents, eyewitness memory researchers and fundamental memory researchers (i.e., those investigating basic memory processes) were targeted. I examined beliefs using a variety of statements concerning the effects of stress on memory. The primary interest of this exploratory survey was to examine what experts from both fields and laypeople believe about these statements. I did not make explicit specific predictions for the survey about current laypeople's and experts' beliefs.

\section{Method}

\section{Participants}

A typical power analysis to determine sample size was not conducted for two reasons: (i) I did not have specific hypotheses for this exploratory survey and (ii) the pool of experts is, naturally, constrained due to the specific nature of expertise. Thus, I based the number of participants on an expected expert response rate estimating with respect to an initial list of experts in relevant areas. I anticipated that I would obtain responses from around 50 eyewitness experts and 50 fundamental memory experts. In the event that such numbers were not forthcoming, the stopping rule was to continue collection for as long as feasible. I planned to recruit a similar number of laypeople, thus aiming for at least 100 layperson participants between the ages of 18 and 65 to best reflect age range in a group of potential American jurors. The survey was pre-registered on OSF (https:// osf.io/b93px?view_only=f83715544c4640c79c3fbfa50d996154). Table 2.3 presents the demographic information for both the final laypeople and expert samples. 


\section{Exclusion criteria}

Four attention checks were included. Specifically, within the instructions, I informed participants that at the end of the survey, they would be asked to choose a shape and that they should select triangle. In addition, there were three unrelated mock statements with a clear answer (e.g., Most humans live more than two hundred years). Participants who failed more than one attention check were excluded. Participants were also excluded if they completed the survey in under 3 minutes. On average, laypeople completed the survey in $9.21 \mathrm{~min}(S D=6.64)$ and experts in $23.37 \mathrm{~min}(S D=19.21)^{1}$.

Laypeople. In total, 129 American participants were recruited using Amazon Mechanical Turk (M-Turk), an online crowdsourcing marketplace. M-Turk has been shown to be a viable platform for academic data collection when compared with other commonly used platforms (Kees et al., 2017). I selected this platform due to the ease and speed of data collection, but also to reach a broad sample of individuals who may indeed be potential jurors (i.e., the general American public).

Laypersons were thanked and received $\$ 1$ as compensation. These data were collected within one week in September 2019.

Experts. Following earlier surveys (Kassin et al., 1989, 2001), I contacted eligible experts, identified by perusing the pertinent literature to find those who had published peerreviewed papers on this topic (i.e., eyewitness and fundamental memory research related to stress and memory). To do so, I searched for combinations of related terms (e.g., stress, arousal, emotion, memory, eyewitness, etc.) on relevant databases (e.g., PsycInfo). Additionally, publications referenced in larger meta-analyses examining stress effects on memory were examined (e.g., Deffenbacher et al., 2004; Shields et al., 2017). Finally, I separately made a list of experts in the field of stress andmemory and searched for additional research that was published by them related to emotion/stress/arousal and memory. One initial email and two follow-up emails were sent to 150 researchers over a four-month period between May and September 2019. Additionally, members of the Society for Applied Research on Memory and Cognition, the European Association of Psychology and Law, the American Psychology-Law Society, and the Stress-NL Consortium $^{2}$ were contacted through server emails, explicitly requesting participation

\footnotetext{
${ }^{1}$ Result reported here excludes three outliers in the expert group (152 hours, 16 hours, and 10 hours) who likely left the survey tab open on their computer during completion over one or several days.

${ }^{2}$ I did not originally include the Stress-NL Consortium in the pre-registered plan for survey dissemination, but decided to distribute the survey to this organization due to its contact with current stress experts.
} 


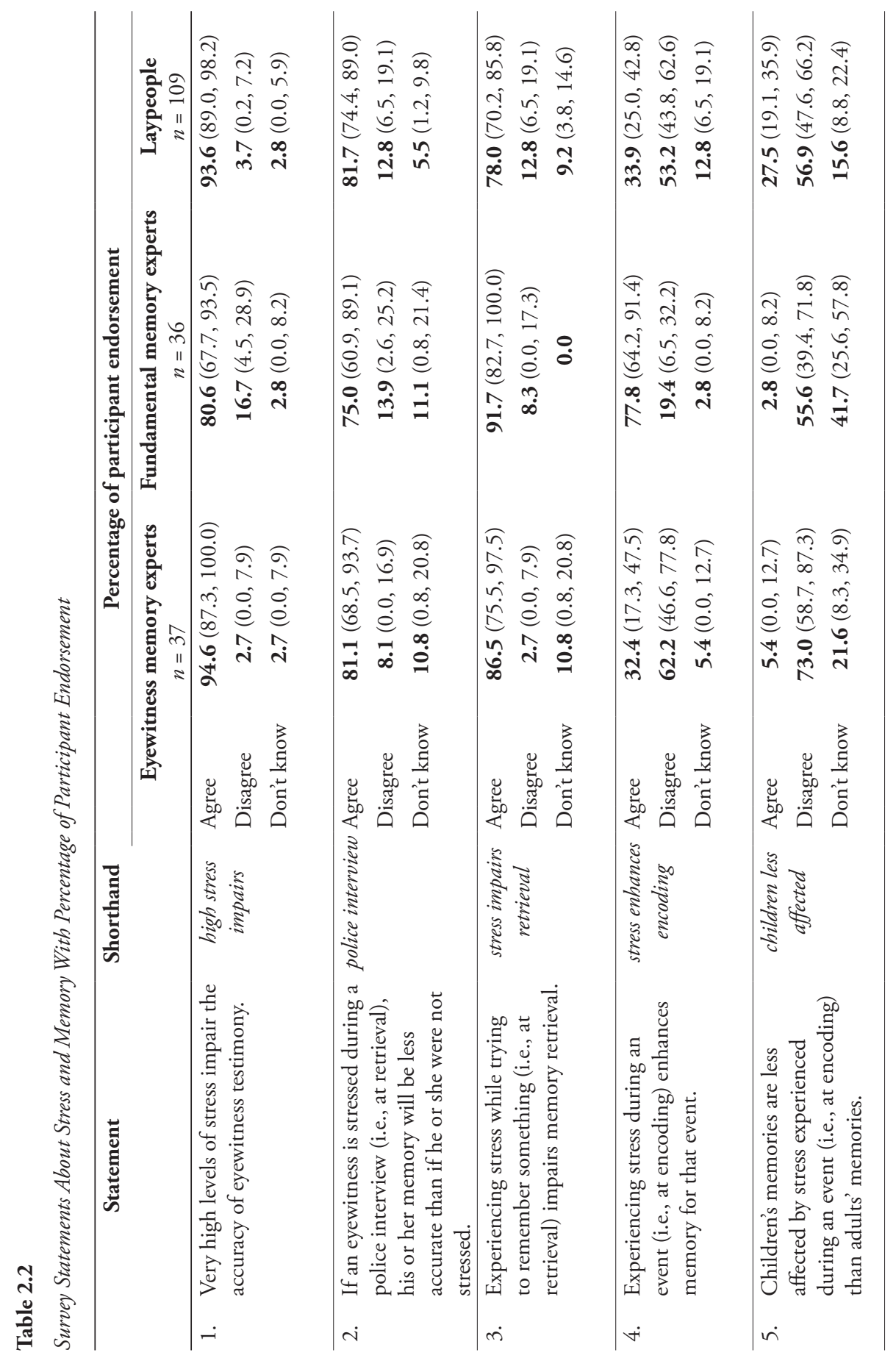




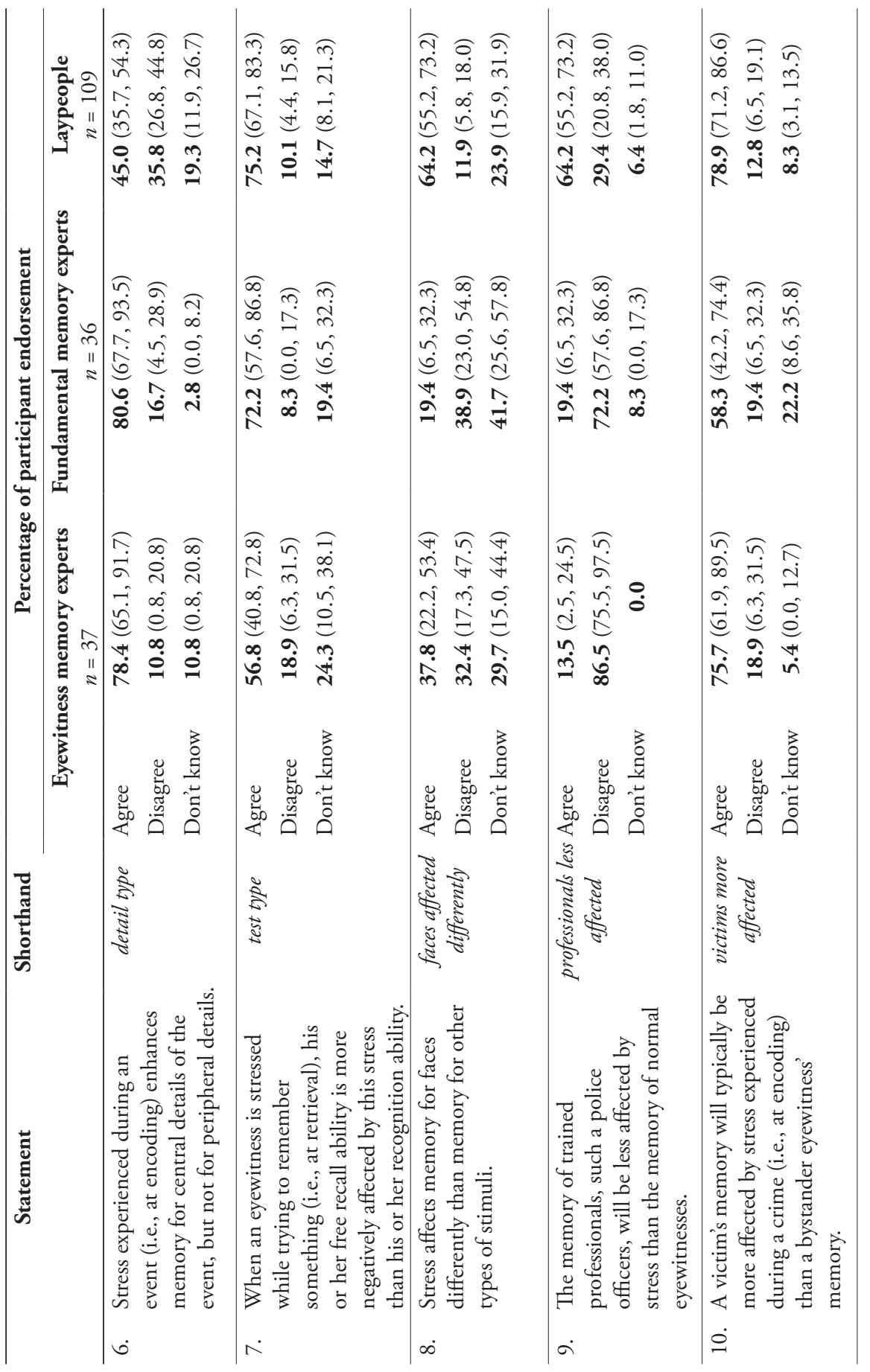




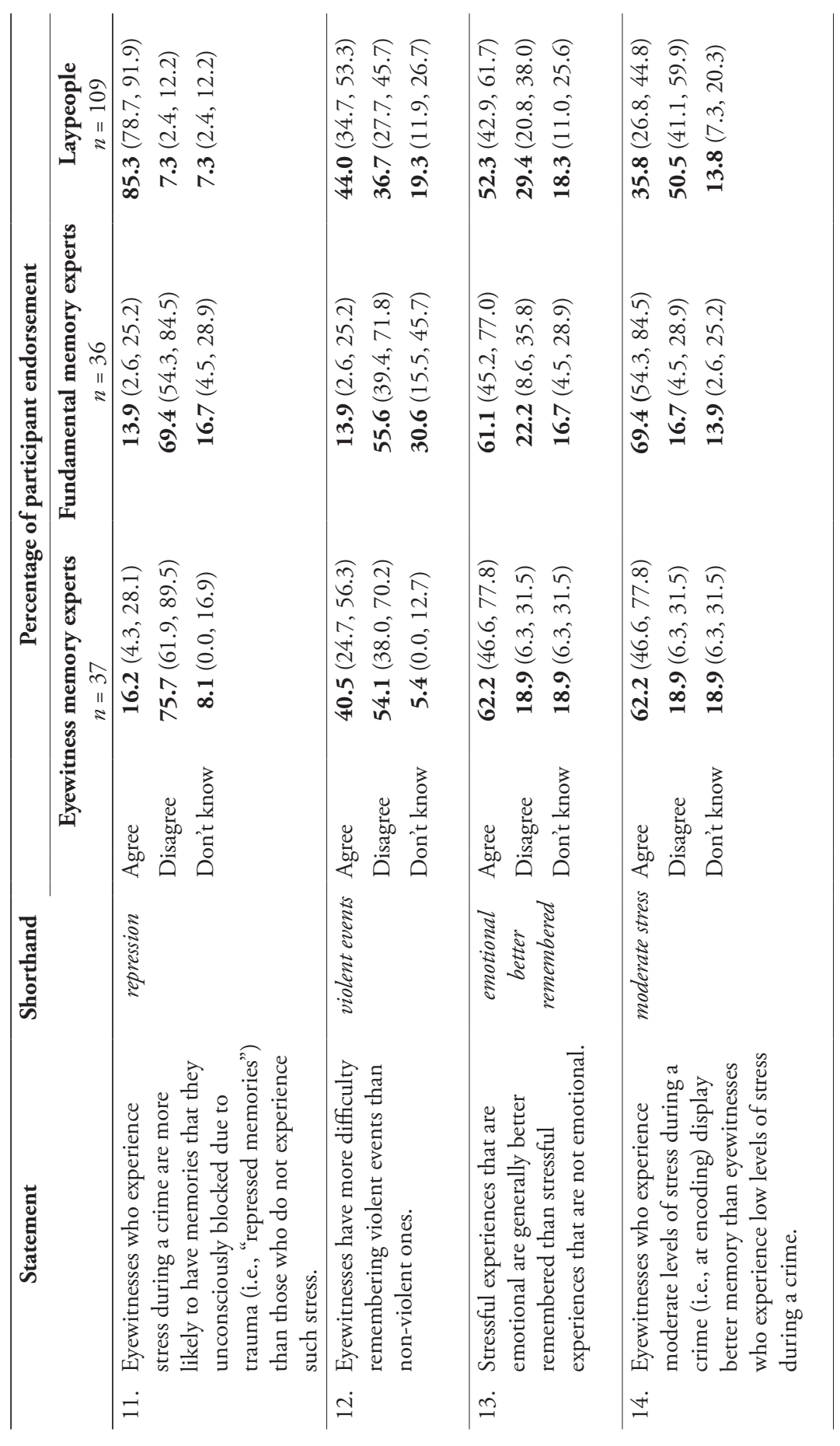




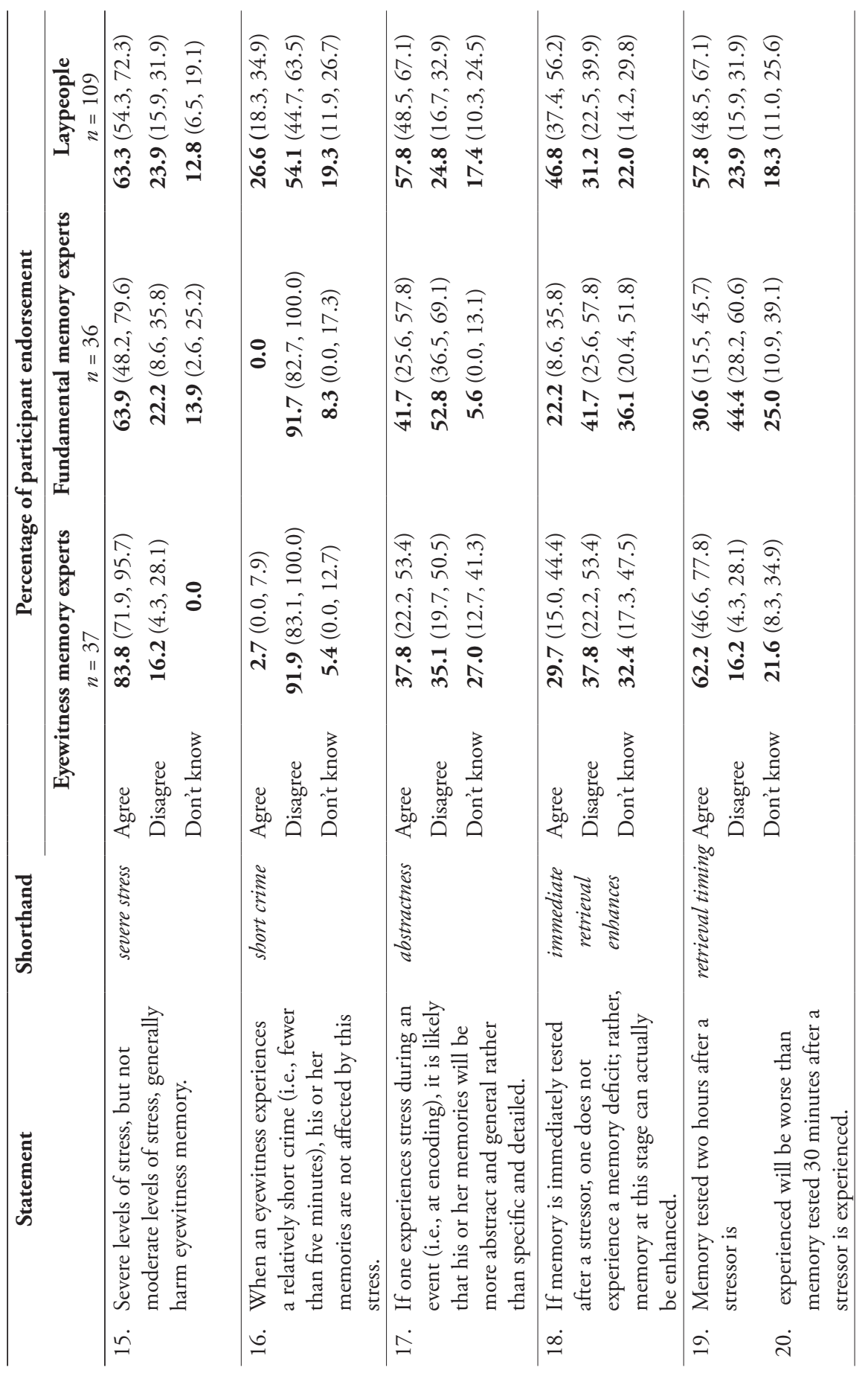




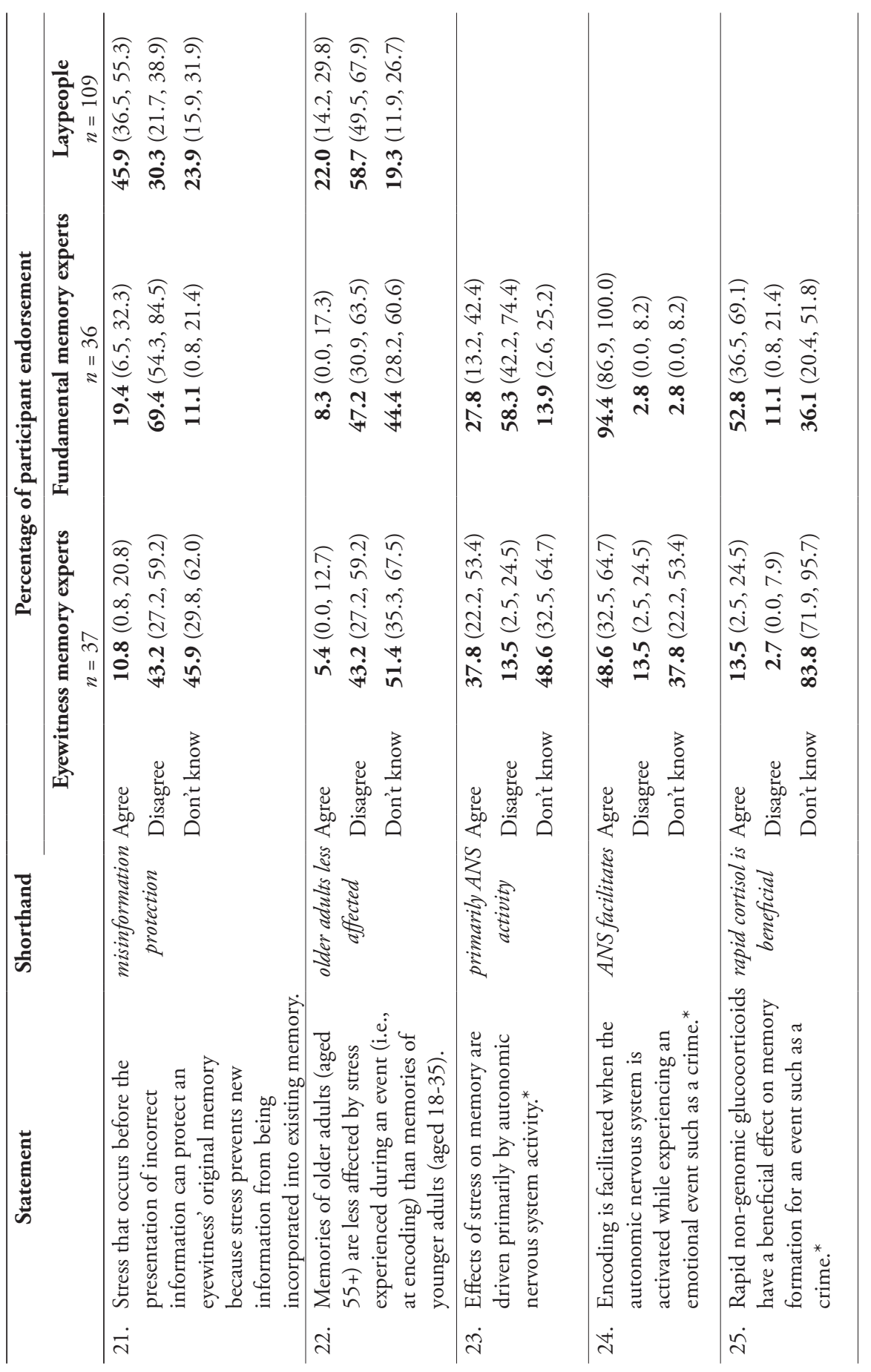




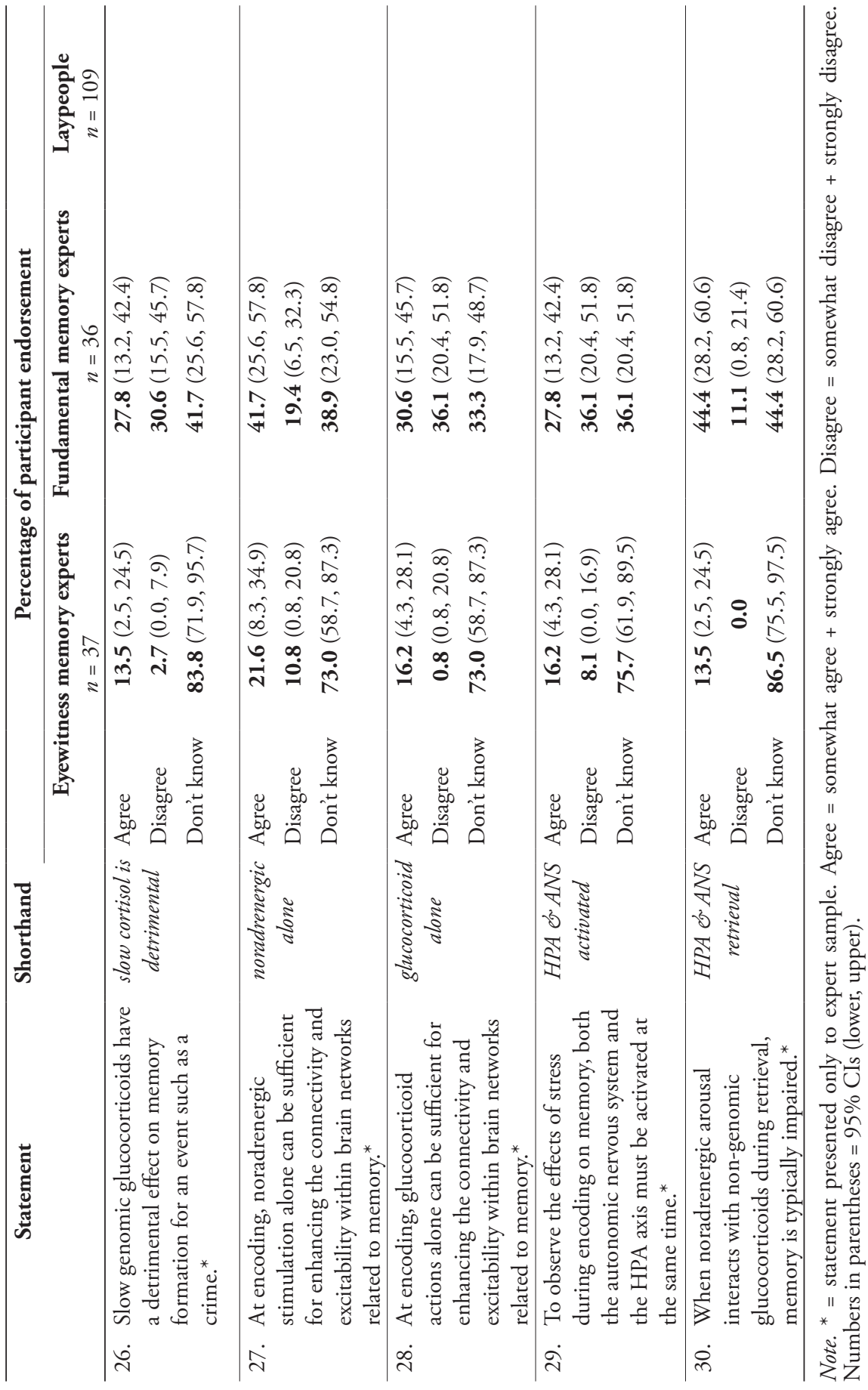


from those who had published peer-reviewed articles on the topic of the effects of stress, arousal, or emotion on memory. The survey was closed in November 2019, after over six months of data collection through these multiple avenues. Out of participants responding to the expert survey, eight did not pass the attention checks and one participant asked to withdraw their data post-survey.

These self-reported experts received additional demographic questions about their research (see Table 2.3). Of the final sample, $89 \%$ possessed a Doctorate degree and the other $11 \%$ held a Master's degree, with 66\% of experts expressing the effects of arousal/ stress on memory as a primary area of interest. Additionally, these experts had published in scientific journals, law reviews, books, chapters, magazines, or newsletters ( $M d n=27$, $I Q R=68$, range $=0$ to 557$)$, many of which focused specifically on the effects of stress on memory $(M d n=4, I Q R=10$, range $=0$ to 400$) .{ }^{3}$ Nearly $29 \%$ of experts had acted as an expert witness, sometimes testifying specifically about the effects of stress on memory $(M d n=5, I Q R=20$, range $=0$ to 500$)$.

If experts classified their primary area of research as eyewitness memory, applied memory in forensic contexts, or other related forensic psychological areas, they were assigned to the eyewitness memory expert group. If experts classified their primary area of research as the neuroscience of memory or another memory or related psychological area, they were assigned to the fundamental memory expert group. Two independent researchers categorized the unclassified research areas, resulting in a high degree of reliability (Koo \& $\mathrm{Li}, 2016)$, ICC (intraclass correlation coefficient; absolute agreement, 2 -way mixed-effects model $)=.866,95 \%$ CI from .709 to $.942, F(21,21)=13.952, p<.001$. Disagreements $(n=1)$ between coders were resolved through discussion. Of the final sample $(N=73)$, 37 were eyewitness experts and 36 fundamental memory experts. Experts were thanked upon completion but received no reimbursement.

\footnotetext{
${ }^{3}$ Analyses were also conducted without the participant reporting zero publications $(n=1)$ and those with missing numerical responses $(n=2)$, and results remained the same.
} 


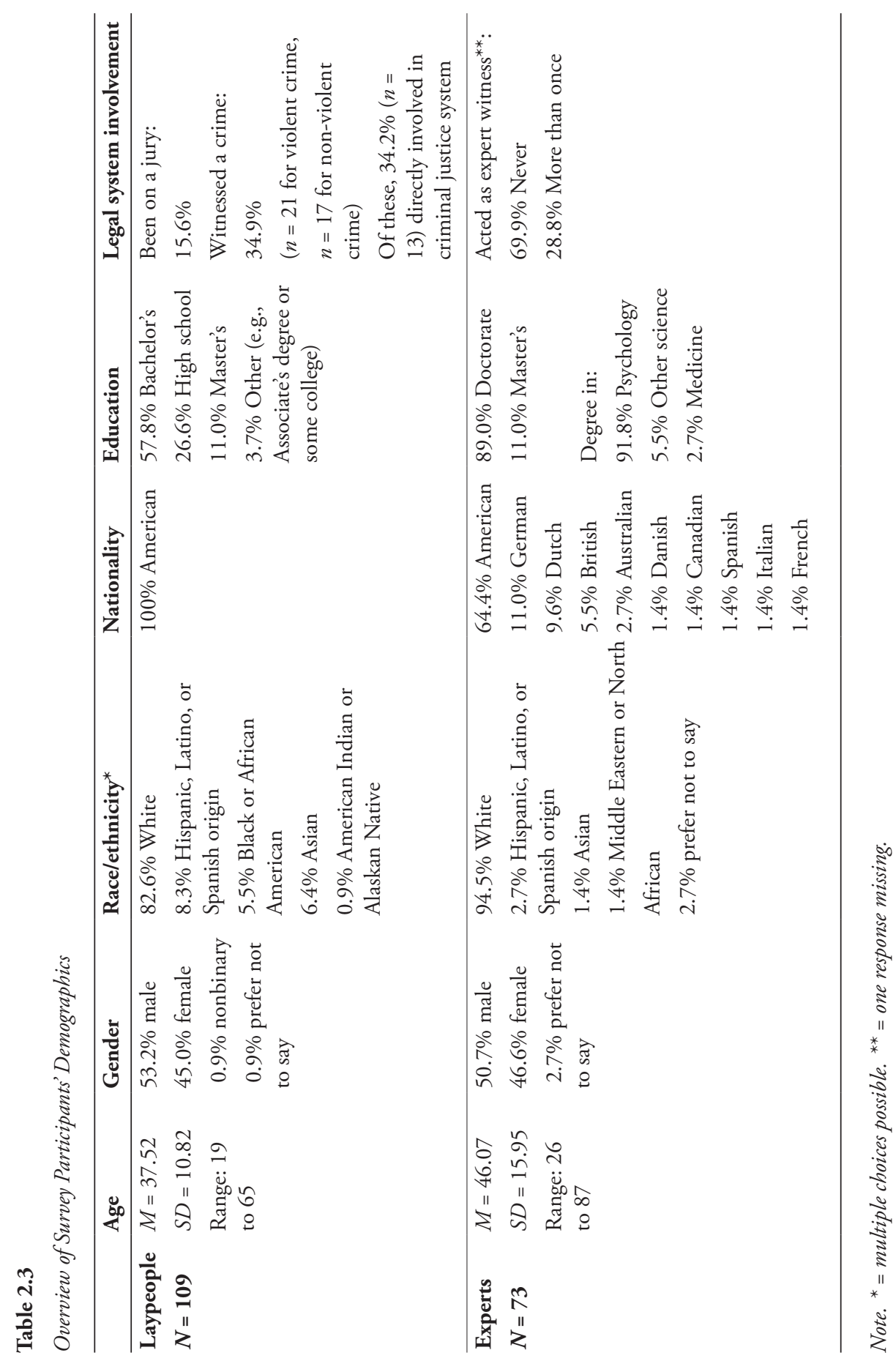




\section{Materials}

\section{Survey}

The survey was created using the online platform Qualtrics (Qualtrics, Provo, UT), with separate versions for laypeople and experts. Both survey versions and datasets are accessible on the OSF, with expert demographic information removed to protect confidentiality (https://osf.io/jpra2/?view_only=a87bc3abda8c4cb699299ecfc9cc94d2). After consenting, participants completed the survey in a self-paced format. They were unable to return to any of the questions once they had continued the survey. For each statement, a shorthand term was created for brevity; Table 2.2 presents these shorthands alongside each statement.

Layperson and Expert Statements. Both survey versions contained the same 21 statements related to the effects of acute stress on (eyewitness) memory. The first fixed statement was a word-for-word reproduction of the single item used in past expert surveys (Kassin et al., 1989, 2001) for comparison purposes (i.e., Very high levels of stress impair the accuracy of eyewitness testimony). I also generated a list of topics relevant to the stress-memory relationship that may be pertinent for eyewitness-related scenarios and are often discussed in relevant reviews or papers (i.e., Christianson, 1992; Deffenbacher et al., 2004; Shields et al., 2017, Shields, 2020). As such, the 20 other statements were generated with reference to past theories or findings about potential effects or moderators of effects or were otherwise relevant to eyewitness-related settings. Specifically, the random-order statements addressed specific issues related to stressor timing on the stressmemory relationship, potential moderators of the stress-memory relationship (e.g., type of memory test, age, role, detail type, stimulus type, stimulus valence), and other areas of interest to the eyewitness field (e.g., misinformation effects, memory specificity, relation to repressed memories). Some of the statements better agreed with the state of the science (e.g., stress impairs retrieval; see Shields et al., 2017), others were less established (e.g., children less affected, see Deffenbacher et al., 2004, for a discussion), debated (e.g., stress enhances encoding) or overlooked in past research (e.g., short crime).

Experts and laypeople were asked to rate each statement from a list of options. Similar to past surveys (e.g., Akhtar et al., 2018; Magnussen et al., 2010; Read \& Desmarais, 2009), experts and laypeople chose from one of five options: strongly disagree, somewhat disagree, somewhat agree, strongly agree, or don't know. Instructions at the beginning of the survey discouraged guessing and clarified to experts that a don't know choice was appropriate 
when the current research in the field is inconclusive. For the final analysis, answers were collapsed and coded as disagree (strongly disagree and somewhat disagree) and agree (strongly agree and somewhat agree; cf. Benton et al., 2006; Read \& Desmarais, 2009). Table A.A in Appendix A shows the distribution of results across all five response categories.

Additional Expert Statements and Questions. The expert version of the survey contained eight additional random-order statements relating to more technical and fundamental topics likely to be unknown and unsuitable for a layperson sample. These statements were generated with reference to current neurobiological theories regarding acute stress effects on memory (e.g., Diamond et al., 2007; Joëls et al., 2006; Quaedflieg \& Schwabe, 2018). Specifically, the statements focused on the most relevant physiological stress responses, addressing the precise roles that the autonomic nervous system and glucocorticoid activity play within the stress-memory relationship.

Additionally, for each statement, experts were asked a) if they believed the statement was reliable enough for psychologists to present in courtroom testimony (yes or no; court reliability), b) if their opinion was based on published, peer reviewed, and scientific research (yes or no; research basis), and c) if they would say that most lay people believe the statement to be true as a matter of common sense (yes, no, or don't know; common sense). Tables A.B and A.C in Appendix A show responses to these additional questions.

\section{Data Analyses}

To address the research questions, I compared (i) expert responses to past expert survey findings, (ii) eyewitness experts to fundamental memory expert responses, and (iii) layperson to expert responses. I conducted a chi-square test between the two groups for each relevant comparison: endorsements, referring to whether participants agreed or disagreed with each statement and selections, referring to whether participants agreed/ disagreed or selected don't know. The preregistered stated that a Bonferroni correction would be used, with the alpha set to $.0017(.05 / 29)$ to correct for multiple comparisons. However, to better preserve power, I instead used a Holm-Bonferroni correction (Holm, 1979) by adjusting p-values based on the number of tests and comparing with an alpha of .05 . 


\section{Results}

\section{Comparison with Past Work}

Following Benton et al. (2006), I compared the proportion of experts who agreed that the statement Very high levels of stress impair the accuracy of eyewitness testimony was reliable enough for psychologists to present in court with data from a past expert survey that used the same statement (Kassin et al., 2001). Although 19 years have passed since the 2001 survey, there is a chance that some of the same experts participated in both surveys, which would violate the assumption of independence for a chi-square test. It is not possible to tell whether this is the case, but due to the possibility, the results of this preregistered chisquare comparison are presented with caution. The current level of endorsement (61\% of experts; i.e., 43 of 71 ) was similar to the previous survey (60\%, i.e., 37 of 62; Kassin et al., 2001), and these endorsement rates did not differ statistically significantly from one another, $\chi^{2}(1, N=133)=0.011, p=.917, \phi=.009$.

\section{Eyewitness Experts versus Fundamental Memory Experts}

Table 2.2 presents expert responses and for each statement, categorized by research field. Figure 2.1 provides a visual overview of agreement rates for each statement between the three groups. I first compared eyewitness memory experts and fundamental memory experts on endorsements (i.e., whether they agreed or disagreed with each statement). Table 2.4 shows the inferential statistics for these comparisons. A statistically significantly difference between groups $(\phi=.462)$ emerged for only one statement, stress enhances encoding. A greater proportion of fundamental memory experts $(77.8 \%)$ than eyewitness memory experts $(32.4 \%)$ agreed with the idea that stress experienced during encoding enhances memory.

For this statement, I also explored relevant data for notable descriptive findings. First, the broader breakdowns of responses across all five categories was examined (reported in Table A.A in Appendix A). The majority of the fundamental memory group selected somewhat agree (63.9\%), with the rest selecting strongly agree (13.9\%) or somewhat disagree (19.4\%), and only one electing don't know (2.8\%). On the other hand, eyewitness experts showed a wider distribution: $8.1 \%$ selected strongly agree, $24.3 \%$ somewhat agree, $37.8 \%$ somewhat disagree, 24.3\% strongly disagree, and 5.4\% don't know. Although the main difference between the groups is clear, examining these broader responses shows the variability in the answers from eyewitness experts in particular. Additionally, I descriptively examined 
proportions for stress enhances encoding only for experts who reported that they had previously testified in court $(n=21$, of which 18 were eyewitness memory experts and 3 were fundamental memory experts).

These experts were mostly eyewitness memory experts (85.7\%), and large diversity in responding to stress enhances encoding in this subgroup is still noticeable. Specifically, experts who had previously testified in court were split on their responses to stress enhances encoding: $14.3 \%$ strongly agreed, $23.8 \%$ somewhat agreed, $42.9 \%$ somewhat disagreed, and $19.0 \%$ strongly disagreed. Next, group differences between eyewitness memory experts and fundamental memory experts on selections were examined (i.e., whether they selected don't know compared to agree or disagree; see Table A.D in Appendix A). Eight statements differed statistically significantly between groups: misinformation protection ( $\phi=.385)$, primarily ANS activity $(\phi=.374)$, ANS facilitates $(\phi=.434)$, rapid cortisol is beneficial $(\phi=.487)$, slow cortisol is detrimental $(\phi=.436)$, glucocorticoid alone $(\phi=.397)$, HPA \& ANS activated ( $\phi=.399)$, and HPA \& ANS retrieval $(\phi=.443)$. For each of these statements, a greater proportion of eyewitness memory experts selected don't know than fundamental memory experts.

\section{Laypeople versus Experts}

Table 2.2 presents layperson responses for each statement. Table 2.5 shows the inferential statistics for the endorsement comparisons between laypeople and experts. The two groups differed statistically significantly in their responses to eight of the statements. A greater proportion of laypeople agreed with the statements compared to experts for six of these statements: children less affected ( $\phi=.301)$, faces affected differently $(\phi=.416)$, professionals less affected $(\phi=.506)$, repression $(\phi=.756)$, short crime $(\phi=.396)$, and misinformation protection $(\phi=.355)$. For detail type $(\phi=.316)$ and moderate stress $(\phi=.366)$, a greater proportion of experts agreed with the statements compared to laypeople. 


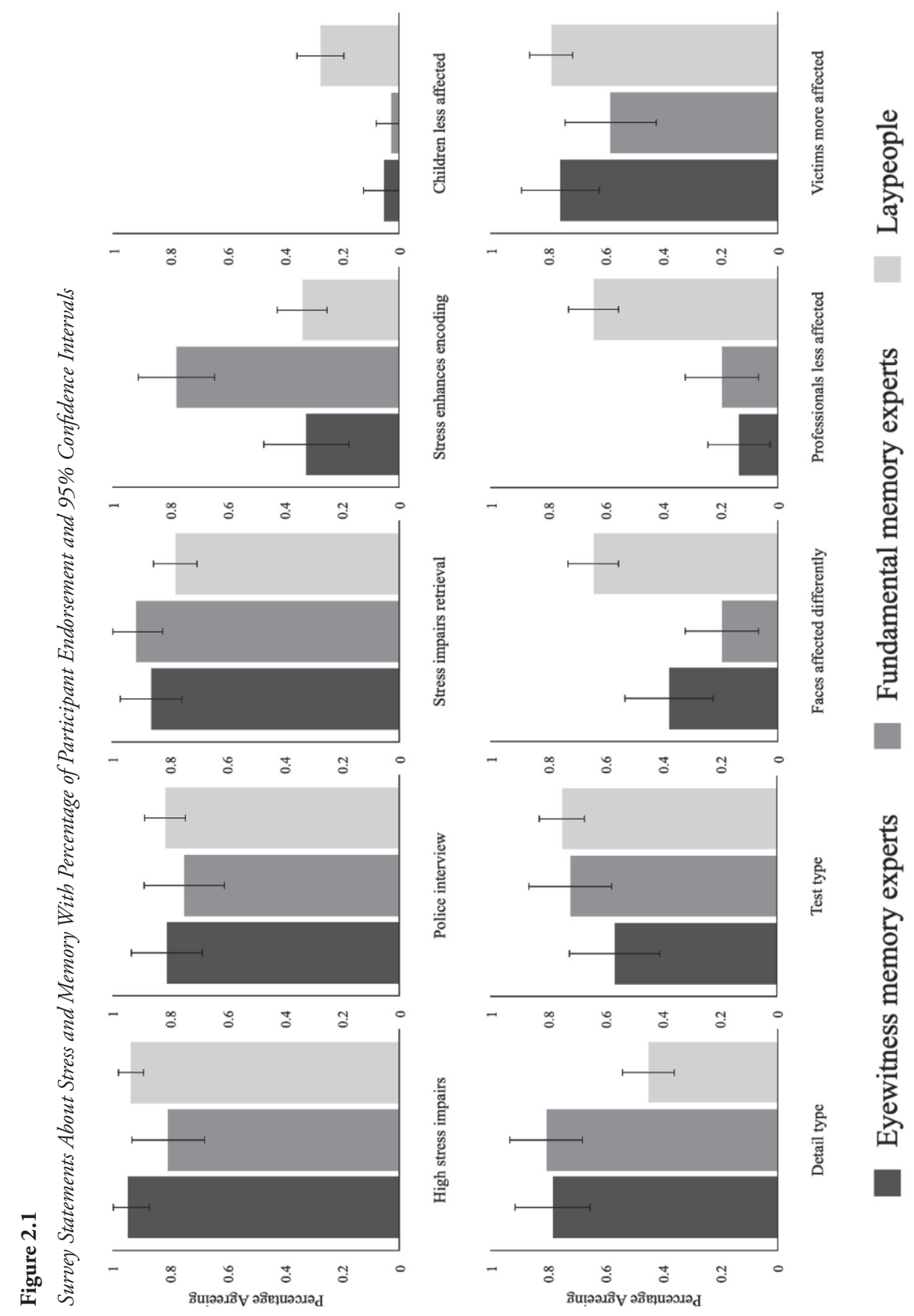




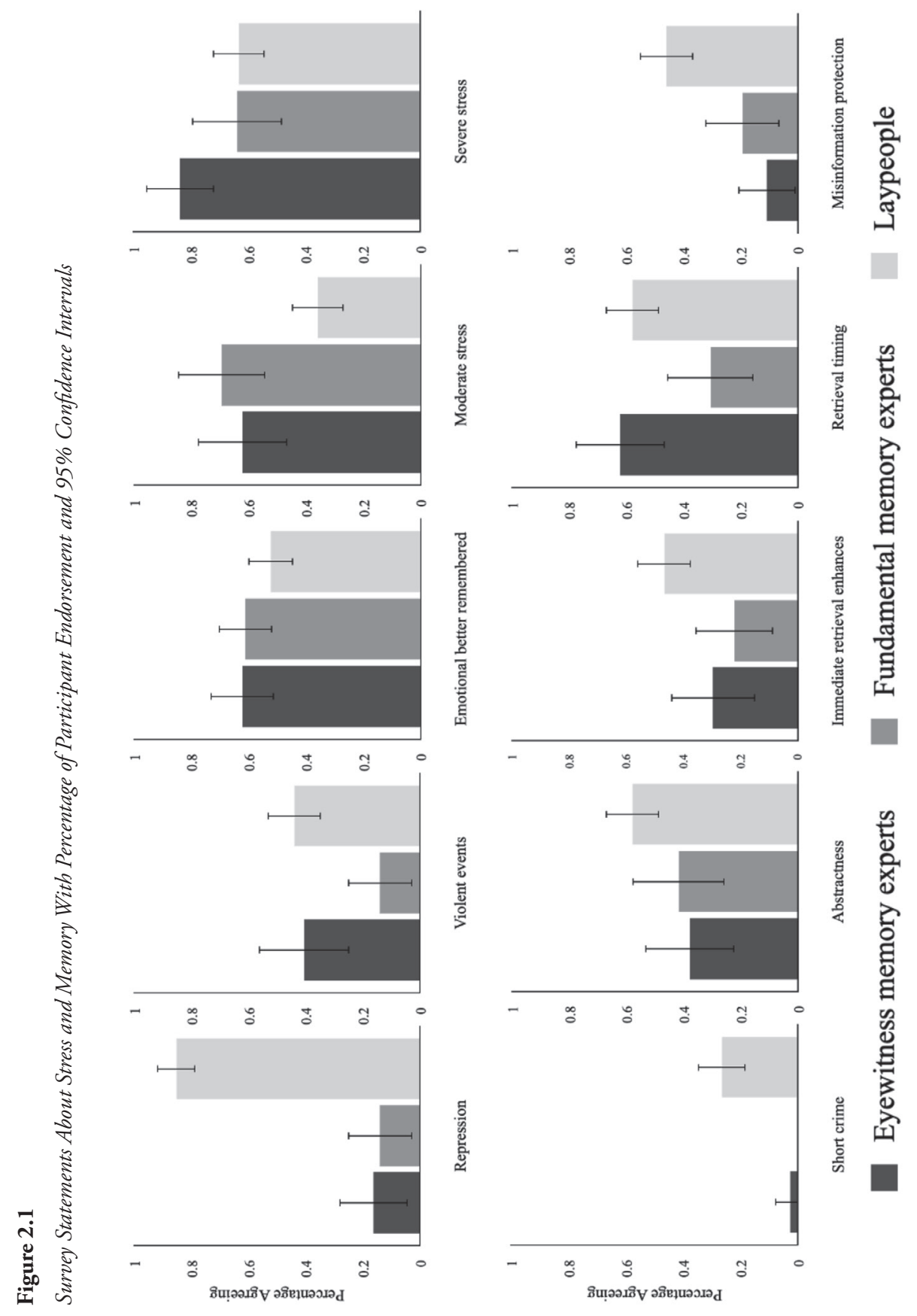




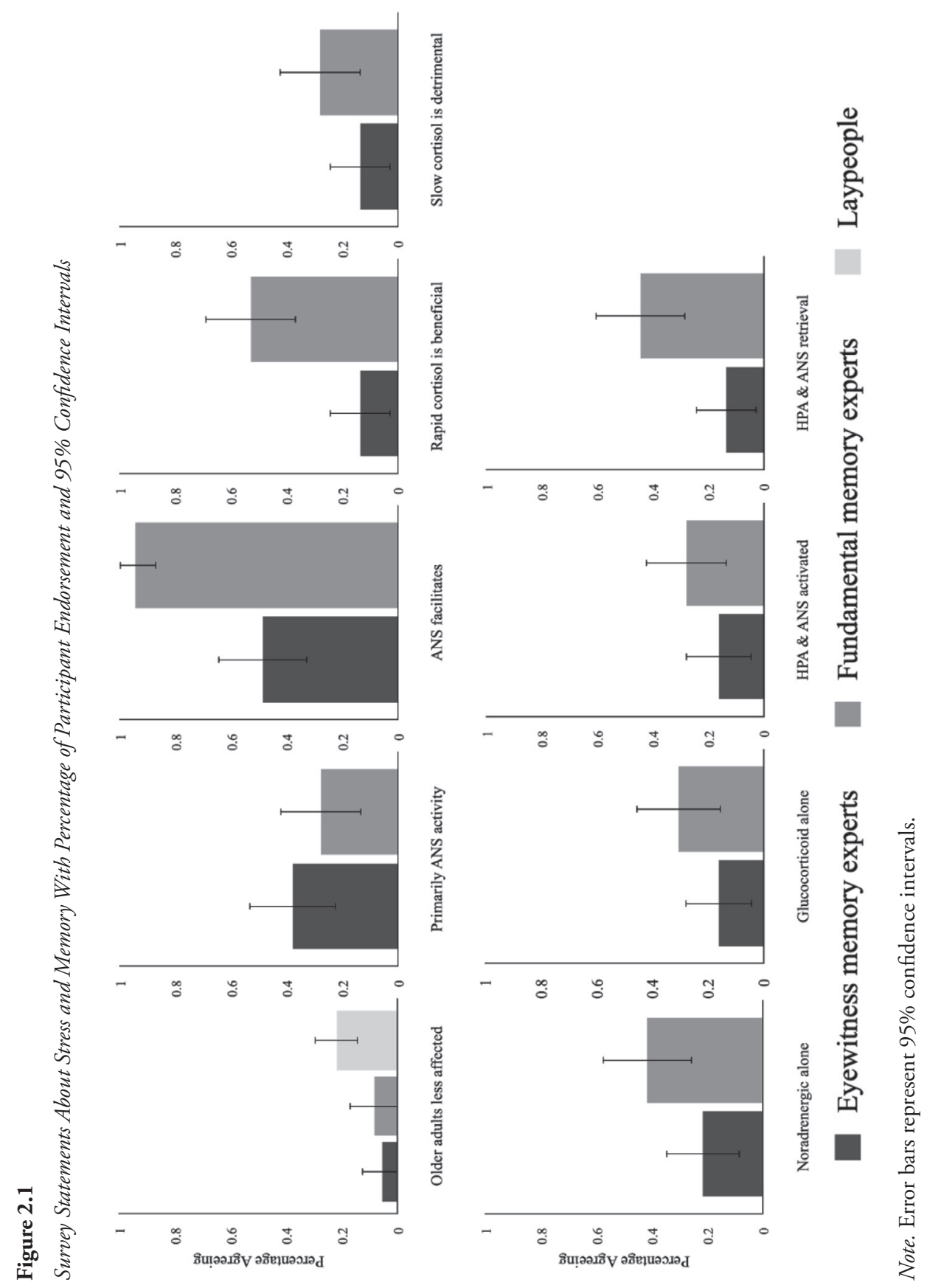


Table 2.4

Inferential Statistics for $2 \times 2 \chi^{2}$ Tests Comparing Endorsements (Agree versus Disagree) in Eyewitness and Fundamental Memory Experts $(d f=1)$

\begin{tabular}{|c|c|c|c|c|c|}
\hline \multirow[b]{2}{*}{ Statement } & \multirow[b]{2}{*}{$n$} & \multirow[b]{2}{*}{$\chi^{2}$} & \multicolumn{3}{|c|}{ Adjusted } \\
\hline & & & $p$ & $p$ & $\phi$ \\
\hline 1. High stress impairs* & 71 & & .055 & .084 & .241 \\
\hline 2. Police interview* & 65 & & .475 & .084 & .099 \\
\hline 3. Stress impairs retrieval* & 69 & & .615 & .084 & .113 \\
\hline 4. Stress enhances encoding & 70 & 14.933 & $<.001$ & $<.001$ & .462 \\
\hline 5. Children less affected* & 50 & & $>.999$ & .084 & .044 \\
\hline 6. Detail type* & 68 & & .735 & .084 & .071 \\
\hline 7. Test type* & 57 & & .179 & .084 & .193 \\
\hline 8. Faces affected differently & 47 & 1.978 & .160 & .084 & .205 \\
\hline 9. Professionals less affected & 70 & 0.728 & .394 & .084 & .102 \\
\hline 10. Victims more affected & 63 & 0.225 & .635 & .084 & .060 \\
\hline 11. Repression & 64 & 0.011 & .917 & .084 & .013 \\
\hline 12. Violent events & 60 & 3.429 & .064 & .084 & .239 \\
\hline $\begin{array}{l}\text { 13. Emotional better } \\
\text { remembered }\end{array}$ & 60 & 0.089 & .766 & .084 & .038 \\
\hline 14. Moderate stress & 61 & 0.144 & .704 & .084 & .049 \\
\hline 15. Severe stress & 68 & 0.949 & .330 & .084 & .118 \\
\hline 16. Short crime* & 68 & & $>.999$ & .084 & .119 \\
\hline 17. Abstractness & 61 & 0.361 & .548 & .084 & .077 \\
\hline $\begin{array}{l}\text { 18. Immediate retrieval } \\
\text { enhances }\end{array}$ & 48 & 0.426 & .514 & .084 & .094 \\
\hline 19. Retrieval timing & 56 & 8.720 & .003 & .084 & .395 \\
\hline $\begin{array}{l}\text { 20. Misinformation } \\
\text { protection* }\end{array}$ & 52 & & $>.999$ & .084 & .022 \\
\hline 21. Older adults less affected* & 38 & & $>.999$ & .084 & .057 \\
\hline 22. Primarily ANS activity & 50 & 8.099 & .004 & .084 & .402 \\
\hline 23. ANS facilitates* & 58 & & .032 & .084 & .303 \\
\hline $\begin{array}{l}\text { 24. Rapid cortisol is } \\
\text { beneficial* }^{*}\end{array}$ & 29 & & $>.999$ & .084 & .008 \\
\hline $\begin{array}{l}\text { 25. Slow cortisol is } \\
\text { detrimental* }\end{array}$ & 27 & & .182 & .084 & .299 \\
\hline 26. Noradrenergic alone* & 33 & 0.567 & $>.999$ & .084 & .047 \\
\hline 27. Glucocorticoid alone & 34 & & .452 & .084 & .129 \\
\hline 28. HPA \& ANS activated* & 32 & & .433 & .084 & .209 \\
\hline 29. HPA \& ANS retrieval* & 25 & & .549 & .084 & .218 \\
\hline
\end{tabular}

Note. Adjusted $p=$ Holm-Bonferroni adjustment for multiple comparisons. Bold $=$ adjusted $p$ significant at the .05 level. * $=$ Fisher's exact test instead of chi-square test (when expected cell sizes $<5$ ). 
Table 2.5

Inferential Statistics for $2 \times 2 \chi^{2}$ Tests Comparing Endorsements (Agree versus Disagree) in Experts and Laypeople $(d f=1)$

\begin{tabular}{|c|c|c|c|c|c|}
\hline \multirow[b]{2}{*}{ Statement } & \multirow{3}{*}{$\frac{n}{177}$} & \multirow[b]{2}{*}{$\chi^{2}$} & \multicolumn{3}{|c|}{ Adjusted $p$} \\
\hline & & & $p$ & & $\phi$ \\
\hline 1. High stress impairs* & & & $>.999$ & .078 & .124 \\
\hline 2. Police interview & 168 & 0.058 & .810 & .078 & .019 \\
\hline 3. Stress impairs retrieval & 168 & 2.959 & .085 & .078 & .133 \\
\hline 4. Stress enhances encoding & 165 & 5.361 & .021 & .078 & .180 \\
\hline 5. Children less affected & 142 & 12.857 & $<.001$ & $<.001$ & .301 \\
\hline 6. $\quad$ Detail type & 156 & 15.613 & $<.001$ & $<.001$ & .316 \\
\hline 7. Test type & 150 & 0.959 & .327 & .078 & .080 \\
\hline 8. Faces affected differently & 130 & 22.472 & $<.001$ & $<.001$ & .416 \\
\hline 9. $\quad$ Professionals less affected & 172 & 44.109 & $<.001$ & $<.001$ & .506 \\
\hline 10. Victims more affected & 163 & 1.837 & .175 & .078 & .106 \\
\hline 11. Repression & 165 & 94.295 & $<.001$ & $<.001$ & .756 \\
\hline 12. Violent events & 148 & 6.463 & .011 & .078 & .209 \\
\hline 13. Emotional better remembered & 149 & 1.992 & .158 & .078 & .116 \\
\hline 14. Moderate stress & 155 & 20.789 & $<.001$ & $<.001$ & .366 \\
\hline 15. Severe stress & 163 & 0.984 & .321 & .078 & .078 \\
\hline 16. Short crime & 156 & 24.480 & $<.001$ & $<.001$ & .396 \\
\hline 17. Abstractness & 151 & 7.704 & .006 & .078 & .226 \\
\hline 18. Immediate retrieval enhances & 134 & 5.612 & .018 & .078 & .205 \\
\hline 19. Retrieval timing & 145 & 1.575 & .210 & .078 & .104 \\
\hline 20. Misinformation protection & 135 & 19.721 & $<.001$ & $<.001$ & .382 \\
\hline 21. Older adults less affected & 126 & 2.984 & .084 & .078 & .154 \\
\hline
\end{tabular}

Note. Adjusted $p=$ Holm-Bonferroni adjustment for multiple comparisons. Bold $=$ adjusted $p$ significant at the .05 level. ${ }^{*}=$ Fisher's exact test instead of chi-square test (when expected cell sizes $<5)$.

For these statistically significant statements, I also descriptively examined responses on a broader scale (Table A.A) to explore noteworthy differences. For example, although $20.2 \%$ of laypeople selected strongly agree for faces affected differently, the majority of experts stuck with a middle category (somewhat agree: $23.3 \%$, somewhat disagree: $28.8 \%$ ) or selected don't know (35.6\%), highlighting the lack of strong expert beliefs about this statement. In addition, $0 \%$ of experts strongly agreed on professionals less affected, while around a quarter of laypeople expressed this extreme agreement (26.6\%). Similarly, for repression, $4.1 \%$ of experts but $33.9 \%$ of laypeople selected strongly agree, while $57.5 \%$ of experts and $1.8 \%$ of laypeople selected strongly disagree. Examining the extreme ends of the broader response scale highlights the extent of the dissimilarity between groups for these statements. 
I also examined differences between layperson and expert selections (see Table A.E in Appendix A). For only one statement did the distribution differ statistically significantly between groups, indicating that a greater proportion of experts selected don't know for the older adults less affected statement than laypeople, $\chi^{2}(1, N=182)=16.88, p<.001$, $\phi=.305$.

\section{Discussion}

In this survey study, I gathered beliefs from memory experts and laypeople related to the effects of stress on eyewitness memory. I was primarily interested in proportions of each group who agreed, disagreed, or selected don't know for each statement (Table 2.2). Additionally, endorsements and selections between groups were compared. In line with previous surveys, most experts in this sample strongly endorsed the belief that high levels of stress impair the accuracy of eyewitness testimony (e.g., Kassin et al., 1989, 2001, Yarmey \& Jones, 1983). In addition, both groups strongly endorsed the statement that stress during retrieval impairs memory, which is in line with findings from fundamental research (e.g., Shields et al., 2017; Wolf, 2017). However, when examining more specific statements in regard to encoding (stress enhances encoding) and retrieval (stress impairs retrieval), results show a divergence between eyewitness and fundamental memory experts. Fundamental memory experts generally agreed that experiencing stress at encoding enhances memory, whereas eyewitness memory experts did not.

Prior research examining the effects of stress during encoding on memory is mixed, with different results often emerging across research fields (e.g., Davis et al., 2019; Deffenbacher et al., 2004, vs. Henckens et al., 2009; Hoscheidt et al., 2004; Vogel \& Schwabe, 2016a). These contrasting findings perhaps account for the contradictory understandings about the effects of encoding stress on memory that emerged in this survey, and are likely due to methodological differences between the research fields. Fundamental memory research tends to use robust experimental methodology including validated laboratory stressors to induce acute stress (e.g., Trier Social Stress Test, Kirschbaum et al., 1993), physiological and subjective manipulation checks to confirm stress inductions, and sufficient retention intervals between sessions (i.e., at least 24-hours) to distinguish the stress effects of encoding and retrieval on memory performance. However, these fundamental studies often examine memory performance for more basic types of stimuli (e.g., word lists, static pictures; Schwabe et al., 2008a; Smeets et al., 2007; Zoladz et al., 2011).

On the other hand, eyewitness memory laboratory research uses unvalidated stressors 
such as violent videos, electric shocks, or self-reports (e.g., Bailis \& Mueller, 1981; Brigham et al., 1983; Clifford \& Hollin, 1981; Kramer et al., 1990). Additionally, many eyewitness experiments rely only on self-reported stress as a manipulation check for the stress induction (e.g., Buckhout et al., 1974; Davis et al., 2019). Indeed, as pointed out by Sauerland et al. (2016), only seven studies included in the Deffenbacher et al. (2004) meta-analysis report physiological stress measures. Subjective reports of stress, however, do not always correlate with physiological acute stress responses (Hellhammer \& Schubert, 2012). Eyewitness field studies show similar limitations, failing to confirm HPA-axis activation (i.e., by examining cortisol) or lacking a sufficient retention interval to specifically examine effects of encoding stress on memory performance (e.g., Hope et al., 2016; Hulse \& Memon, 2006; Morgan et al., 2004; Valentine \& Mesout, 2008). The single session designs often used in eyewitness memory research make it impossible to isolate the effects of encoding stress on different memory phases (i.e., consolidation versus retrieval; Sauerland et al., 2016; Thomas \& Karanian, 2019).

Many of these methodological differences between fields stem from the distinct goals of each particular research field. While the fundamental memory field often aims to examine the basic neurobiological activities underlying the stress-memory relationship, the eyewitness memory field is more interested in the impact that acute stress can have on memory for a crime in applied witness contexts. Thus, the eyewitness memory field notably attempts to mimic witness experiences. However, in such applied experiments, isolating stress effects can be difficult, sometimes leading to a mischaracterization and overgeneralization of the term acute stress (i.e., a physiological response involving HPA axis activation, as defined in the fundamental memory field). Stemming from these unique research aims, the varied methodology between fields likely contributes to the contrasting results, and perhaps explains why experts from the two fields often express opposing views about how encoding stress affects memory performance. This divergence in perspectives suggests an absence of interactions between research fields. Critically, understanding results from fundamental memory studies that use more precise methodology might be useful for eyewitness experts. Eyewitness researchers examining the effects of stress on memory performance should strive to gain knowledge about the fundamental stress literature and the methodological gold standards (see Shields, 2020), and should also aim to collaborate with fundamental stress experts. In addition, fundamental memory researchers could conduct research alongside or in consultation with eyewitness memory researchers to produce work that better reflects conditions in the real world, for example by using more ecologically valid scenarios (e.g., mock crimes). 


\section{Expert Beliefs about Moderators Between Encoding Stress and Memory}

To better parse responses to the more general statements about encoding stress effects on memory performance, I also probed for experts' beliefs about potential moderating factors that may affect the relationship between encoding stress and memory. Many of the statements that showed low levels of expert endorsement (i.e., below 50\%; abstractness, faces affected differently, violent events, children less affected, older adults less affected) have not been thoroughly empirically tested. For example, although some findings indicate that children (Deffenbacher et al., 2004) or older adults (Hidalgo et al., 2019; Smith et al., 2019) may be less affected by stress than younger adults, the vast majority of studies have focused solely on younger adults. Therefore, the lack of consensus and higher levels of don't know responses are in line with available research findings. These data may help guide future research by emphasizing some of the moderators that need to be further examined with empirical work. However, some statements received high levels of endorsement despite ambiguity in research findings. There are conflicting findings regarding differences in stress effects on victims versus bystander eyewitnesses (e.g., Hope et al., 2016; Hosch \& Bothwell, 1990; Kassin, 1984), yet most of the eyewitness memory experts and fundamental memory experts endorsed the idea that a victim's memory will be more affected by encoding stress (victims more affected). Similarly, the vast majority of both expert groups disagreed that stress experienced during a short crime will not affect memories (short crime), although I have not been able to identify any empirical research conducted on this specific topic. Furthermore, both groups generally agreed that eyewitnesses who experience moderate levels of stress during a crime display better levels of memory than those who experience low levels of stress (moderate stress). Most experts from both groups also endorsed the idea that severe but not moderate levels of stress generally harm eyewitness memory (severe stress). Neuroscientific research supports this inverted-U shape idea, which suggests poorer cognitive performance at low and high levels of stress and better performance at medium levels of stress (e.g., Abercrombie et al., 2003; de Kloet et al., 1999; Lupien et al., 2007). This inverted-U might also explain the different findings between the eyewitness and fundamental memory fields. For example, some fundamental memory research suggests that stress induced in the laboratory during encoding enhances stressor-related memory (e.g., Vogel \& Schwabe, 2016a), while field studies have found impairments in stressor-related memory (e.g., Metcalfe et al., 2019). Although research directly supporting the statements discussed in this section is not substantial, experts might have drawn from relevant theories to support their choices on these topics (e.g., dual-mode model, temporal dynamics model, Yerkes- 
Dodson law; Diamond et al., 2007; Joëls et al., 2006; Yerkes \& Dodson, 1908). Empirical work on these generally endorsed but under-researched topics would also be beneficial for understanding the intricacies of the stress-memory relationship.

Other relevant factors endorsed by experts have a more solid research evidence-base. For example, both expert groups agreed that emotional stressful experiences are remembered better than non-emotional ones (emotional better remembered), an account supported by research (Cahill et al., 2003; Kuhlmann et al., 2005b; Shields et al., 2017, but see Schwabe et al., 2008a; Shermohammed et al., 2019). Additionally, both agreed that encoding stress enhances memory for central details and undermines memory for peripheral details (detail type). These opinions are generally supported by research that suggests simultaneous helping and harming effects of stress on different types of details (Christianson, 1992; Christianson \& Loftus, 1987; Heuer \& Reisberg, 1990, but see Lanciano \& Curci, 2011; Wessel et al., 2000). Eyewitness memory experts likely related this statement to the weapon focus effect (e.g., Kramer et al., 1990; Loftus et al., 1987), a phenomenon demonstrating that eyewitness memory for faces and other details is poorer if a weapon was present during a crime (Fawcett et al., 2013). Finally, the majority of both groups disagreed that those who experience stress are more likely to have repressed memories than those who do not (repression), which is in fact not supported by empirical data (e.g., Otgaar et al., 2019).

\section{Expert Beliefs about Moderators between Retrieval Stress and Memory}

I also examined factors relevant to stress effects at memory retrieval. The majority of experts who agreed that retrieval stress impairs memory also endorsed a more applied version of this statement, though to a lesser extent. This more applied statement (police interview) stems logically from the broader statement (stress impairs retrieval), though specific research has not yet been conducted on this topic. Other retrieval-related statements were based on limited prior research. For example, some research suggests that free recall is impaired more than recognition ability by stress before retrieval (test type; de Quervain et al., 2000, 2003; Gagnon \& Wagner, 2016), a statement generally endorsed by both groups. Some experimental results also suggest that if memory is tested immediately after a stressor, memory is not harmed but rather sometimes even enhanced (immediate retrieval enhances; Schönfeld et al., 2014; Schwabe \& Wolf; 2014). However, less than a third of both expert groups agreed. Finally, around two thirds of eyewitness memory experts and one third of fundamental memory experts believed that memory 
tested two hours after a stressor will be worse than memory tested 30 minutes after a stressor (retrieval timing), a statement based on some limited results (e.g., Schwabe \& Wolf, 2014). These statements have some basis in research but lack a substantial literature, which may explain the absence of expert consensus in this sample.

\section{Expert Beliefs on Neuroscientific Statements}

Experts answered eight additional statements about neuroscientific explanations of stress effects on memory. These statements were mostly based on theoretical research (e.g., Diamond et al., 2007; Joëls et al., 2006, 2011; Quaedflieg \& Schwabe, 2018; Roozendaal, 2002; Schwabe et al., 2012) that delineate the specific timing and roles autonomic nervous system and glucocorticoid activity play in the relationship between stress and memory. Overall, eyewitness memory experts selected don't know more often than fundamental memory experts for each statement. This disparity between expert groups suggests that eyewitness memory experts understand less about the neuroscience behind the stress-memory relationship. That being said, perhaps most striking in regard to the eight neuroscientific statements was the proportion of don't know selections across both groups of experts. Over a third of fundamental experts also selected don't know for most of the neuroscientific statements. Some statements had a more limited research basis, including statements about how noradrenergic stimulation and glucocorticoid activation act specifically alone or together to affect brain networks related to memory (noradrenergic alone, glucocorticoid alone, HPA \& ANS activated). Other statements were more established (see Joëls et al., 2006; Quaedflieg \& Schwabe, 2018), but did not receive a majority endorsement from fundamental memory experts (slow cortisol is detrimental, HPA \& ANS retrieval). The proportion of don't know selections indicate a lack of knowledge in this research area, suggesting that certain topics are not yet established and accepted by an expert majority - at least in these two research domains.

A majority of fundamental memory experts did generally show consensus on three statements, which point towards research findings that are more accepted. Fundamental memory experts mostly disagreed that effects of stress on memory are primarily driven by autonomic nervous system activity, though nearly all agreed that encoding is facilitated when the autonomic nervous system is activated while experiencing an emotional event such as a crime. Additionally, most fundamental memory experts agreed that rapid nongenomic glucocorticoids have a beneficial effect on memory formation. Considering that $78 \%$ of fundamental memory experts agreed that experiencing encoding stress enhances 
memory, these endorsements of neuroscientific explanations of encoding enhancements are perhaps unsurprising.

\section{Layperson Beliefs about Stress Effects on Memory}

As juror opinions about stress effects on memory can also enter the courtroom and may affect decision-making (Bornstein et al., 2008), I examined laypeople's responses and compared them to experts' responses. In line with experts, most laypeople agreed that high levels of stress impair eyewitness testimony (high stress impairs). In contrast to experts, only about a third of laypeople believed that moderate levels of stress at encoding could enhance memory compared to low levels of stress (moderate stress). Thus, laypeople tend to view stress as overwhelmingly negative, with any degree of stress in any memory phase generally impairing memory.

Other differences between laypeople's and experts' responses point towards diverging opinions of the public, including the controversial belief that stress causes repressed memories (repression, 85\%), which research suggests is not the case (e.g., Otgaar et al., 2019). Additionally, the majority of laypeople believed that police officers' memories are resistant to stress effects while eyewitness and fundamental memory experts did not (professionals less affected), a view more in line with the limited research on this topic (e.g., Stanny \& Johnson, 2000). Finally, laypeople also believed that stress affects faces differently than other types of stimuli (faces affected differently) contrasting lower endorsement levels from eyewitness memory experts and fundamental memory researchers on this underresearched and inconclusive topic.

Whereas expert beliefs are generally formed from research on these topics in academic settings, laypersons' beliefs likely stem from intuitive feelings or perceptions about each statement. Given that stress is generally viewed as a negative experience (e.g., Adams, 2016; Becker, 2013), it is unsurprising that laypeople seem to view any degree of stress as harmful, in contrast with expert opinion. Laypeople's agreement that police officers' memories can withstand stress is also an evident erroneous but understandable commonsense belief (e.g., Hope, 2016; Stanny \& Johnson, 2000). A related statement used in past surveys showed that low percentages of laypeople (28\% and 39\%) endorsed the idea that Police officers and other trained observers are no more accurate as eyewitnesses than is the average person (e.g., $N=111$, Benton et al., 2006; $N=79$, Kassin \& Barndollar, 2001, respectively). Taken together, these responses suggest that many laypeople believe that professionals are generally better eyewitnesses who are less influenced by 
external factors such as stress. Two recent surveys also show that large proportions of participants (59\% and 67\%, respectively) endorsed the idea that traumatic experiences can be unconsciously repressed for many years and then recovered $(N=230$ and $N=79$; Otgaar et al., 2019), a statement similar to repression in the current survey. Factors such as television and media may influence such beliefs. For example, $75 \%$ of students $(N=613)$ who reported hearing about someone recovering a repressed memory said they heard about such a circumstance though television (Golding et al., 1996). Additionally, amount of media exposure to information about repressed memories was positively correlated with beliefs in repressed memories. Thus, perhaps the endorsement of the idea that stress causes repressed memories from laypeople in this sample originally stemmed from media or television exposure. To sum up, as demonstrated in this survey and previous surveys, commonsense beliefs do not always align with expert assessments concerning what the contemporary science suggests (e.g., Benton et al., 2006; Simons \& Chabris, 2011).

\section{Implications for Applied Legal Settings}

These data serve as an initial empirical attempt to examine expert and layperson beliefs about the effects of acute stress on memory performance. Although agreement between expert groups was observed on several statements, the most striking difference between groups pertained to the statement that stress enhances encoding $(\mathbb{Q}=.462)$, where fundamental memory experts mostly agreed and eyewitness memory experts mostly disagreed. As seen in an exploratory analysis, there was also a descriptive split among experts who had testified in court. That is, not all testifying experts fell on one side of the belief (i.e., agreeing vs. disagreeing with the statement that stress enhances encoding). These results further support the idea that different expert witnesses bring different views into the courtroom. In this way, jurors and judges could hear contrasting statements from opposing expert witnesses, or hear from only one expert witness, who could fall on either side of the belief. If an expert witness strongly endorses the idea that encoding stress enhances memory, jurors could assume that testimony provided by an eyewitness who experienced stress is highly reliable. If an expert witness reports the opposite, jurors may unreasonably disregard the testimony of an eyewitness who experienced stress. Thus, for seemingly irresolute statements such as stress enhances encoding, exercising caution in the courtroom is important.

On the other hand, these data also suggest that jurors already likely bring their own conceptions about the effects of stress on memory performance into the courtroom. 
Understanding these pre-existing commonsense beliefs is crucial for knowing where expert witness knowledge is needed. For example, if laypeople assume that any amount of stress will automatically impair memory, they may view the testimony from a stressed eyewitness as lacking in probative value. This could later affect their legal decisions. Similarly, if laypeople are unaware of how stress can affect the memories of professionals such as police officers, they may give too much credence to their testimonies over others. For topics like these that show greater consensus from experts in general, reports from an expert witness could be particularly valuable in the courtroom.

\section{Limitations and Future Directions}

There are a number of limitations associated with the current survey. Some nuance is lost when using closed statements with an agree/disagree response format that force respondents to 'choose a side'. Particularly, responding to broad statements (e.g., high stress impairs, stress enhances encoding, stress impairs retrieval, etc.) that use wide-ranging terms such as "memory" can be challenging. That is, "memory" could be thought of quite generally, for example knowing that an experience occurred (e.g., ability to remember gist or central information) or much more specifically (e.g., ability to remember detailed or peripheral information). For this reason, I included several other statements to provide us with more insight into potential moderators that could explain differences in how the broader statements were answered (e.g., severe stress, detail type, abstractness, etc.). With these more specific statements, in contrast to previous surveys, I was better able to interpret the results than if broad generalizations alone were used. Future research could explore the use of a less rigid method such as a qualitative survey or perhaps focus groups, to examine the specific circumstances in which experts believe stress enhances, impairs, or does not affect memory.

A potential limitation pertaining to the layperson group is that the statements might have been too technical for them. I initially addressed this by leaving out the technical neuroscientific statements for the lay sample (i.e., items 22-29), and by explicitly defining certain memory jargon such as terms like encoding and retrieval. However, other terms may have also been too technical for them to fully understand the statements, such as understanding the meaning behind central vs. peripheral details or free recall vs. recognition. Given the laypeople's responses for these statements, it does not seem that much concern is warranted, although future research should aid layperson understanding as much as possible in surveys. 
The expert sample size in this survey is comparable to past expert surveys (e.g., Kassin et al., 1989, 2001). Nonetheless, analyses comparing expert groups might be underpowered due to the limited number of experts who were involved in the survey. Obtaining this expert sample was difficult due to the inherently limited population and I collected expert data for six months using multiple channels and repeated calls in an attempt to access the largest sample possible. I also attempted to reduce the Type 1 error by using a Holm-Bonferroni correction. With a final sample of 73 experts, the between-expert comparisons are only powered to detect large effects (80\% powered to detect Cohen's $w$ $=.50)$, whereas the expert-layperson analyses $(n=182)$ are powered to detect medium and large samples $(80 \%$ powered to detect Cohen's $w=.30)$. Thus, results should be interpreted with caution, keeping the limited sample size and number of comparisons in mind. Specifically, owing to the reduced power, non-statistically significant differences may have been due to an inability detect smaller effect sizes, and the statistically significant differences may be overestimated. On the other hand, large effects may be most relevant for real world application in this area.

In addition to a greater number of experts, a more comprehensive representation of possible experts would also benefit future research. This study examined only a subsection of potential experts: academics who investigate stress effects on memory or related topics. Nearly a third of experts in this survey have experience acting as expert witnesses in court. However, other categories of people who also testify in court settings as experts (e.g., clinical psychologists) may not publish on these matters. This survey did not include those various groups, and thus, the definition of expert in the current survey is restrained to those working and publishing in academic contexts.

The results from this survey might serve as a beneficial guide for future research in this area. Statements that experts answered with don't know or where agree and disagree selections were divided may indicate areas that need to be better investigated. These areas include factors such as age differences (children less affected, older adults less affected), specificity of stressor timing (immediate retrieval enhances, retrieval timing), factors at encoding (violent events, moderate stress), and the form of remembered information (test type, faces affected differently). The results also show the continuing need for fundamental neuroscientific research about how biological stress responses affect memory formation and retrieval in humans. Finally, I suggest that academic experts should be aware of research that exists across the wider research domain, particularly if they plan to testify in court on these matters. 


\section{Conclusion}

This survey explored contemporary experts' and laypeople's beliefs about the effects of stress on memory encoding and retrieval. Only five statements (i.e., high stress impairs, stress impairs retrieval, police interview, detail type, and short crime) out of 29 received consensus levels of over $75 \%$ among both eyewitness and fundamental memory expert groups. As such, these results appear to indicate a general lack of consensus about most factors that play a role in the stress-memory relationship. However, the two expert groups only statistically differed from each other regarding the enhancing effects of encoding stress on memory. Examining beliefs about other factors, such as stress severity and type of remembered detail, provided some insight into this disparity. Laypeople differed from experts on some factors and endorsed some ideas that are not supported by empirical research, for example, that trained professionals such as police are less affected by stress and that stress causes repressed memories. In summary, results from this survey suggest that whereas some factors have a wide consensus among experts, there may be significant gaps in this literature where more research is needed to enhance our understanding of the relationship between stress and memory. 


\section{CHAPTER 3}

\section{Examining the Effects of Acute Stress at Encoding and Retrieval on Face Recognition Memory}

This chapter is based on the following manuscript:

Marr, C., Quaedflieg, C.W.E.M., Otgaar, H., Hope, L., \& Sauerland, M. (2021). Facing stress: Examining the effect of acute stress during encoding and retrieval on face recognition. Acta Psychologica, 219(103376). https://doi.org/10.1016/j. 


\begin{abstract}
Eyewitnesses may experience stress during a crime and when attempting to identify the perpetrator subsequently. Laboratory studies can provide insight into how acute stress at encoding and retrieval affects memory performance. However, previous findings exploring this issue have been mixed. Across two preregistered experiments, I examined the effects of stress during encoding and retrieval on face and word recognition performance. I used the Maastricht Acute Stress Test (MAST) to induce stress and verified the success of the stress manipulation with blood pressure measures, salivary cortisol levels, and negative affect scores. To examine differences in stressor timing, participants encoded target faces or words both when confronted with the stressor and during the subsequent cortisol peak and retrieved these stimuli 24-hours later. I found neither effects of acute stress on face recognition memory during encoding or retrieval (Experiments 2 and 3), nor effects of encoding stress on word recognition memory (Experiment 3). Bayesian analyses largely provided substantial or strong evidence for the null hypotheses. I emphasize the need for well-powered experiments using contemporary methodology for a more complete understanding of the effect of acute stress on face recognition memory.
\end{abstract}




\section{Introduction}

The legal system often relies on eyewitnesses to identify perpetrators in the course of criminal investigations. Eyewitnesses may experience stress during a crime and may feel stressed when attempting to identify the perpetrator at the police station (e.g., Bornstein et al., 2013; Yuille \& Cutshall, 1986). It is known that acute stress at retrieval negatively affects episodic memory performance (Shields et al., 2017; Wolf, 2017), but these effects are understudied in tasks with more applied eyewitness relevance, such as face recognition. In addition, laboratory experiments examining the effects of acute stress at encoding on episodic memory performance often show mixed findings (e.g., Deffenbacher et al., 2004; Shields et al., 2017; Vogel \& Schwabe, 2016). Thus, it remains unclear how acute stress experienced at encoding or retrieval might affect witness face recognition performance.

\section{Effects of Acute Stress at Encoding on Memory Performance}

Whether acute stress during encoding has a negative or positive effect on memory performance seems to depend on the methodology used within a research discipline. In the eyewitness field, most research assessing measures such as recognition accuracy and discriminability reports that acute stress impairs memory performance by (e.g., Davis et al., 2019; Deffenbacher et al., 2004; Morgan et al., 2004). In contrast, in the fundamental memory field many studies demonstrate that acute stress at encoding can enhance recognition accuracy and discriminability (e.g., Henckens et al., 2009; Vogel \& Schwabe, 2016; Zoladz et al., 2011), although meta-analytic results suggest such enhancements occur only under certain conditions (e.g., for experiments using stressorrelevant materials and a short delay between stressor and encoding; Shields et al., 2017). These discrepant findings align with expert views on how experienced stress at encoding might affect memory. One recent survey showed that $78 \%$ of 36 fundamental memory experts agreed that Experiencing stress during an event (i.e., at encoding) enhances memory for that event, whereas only $32 \%$ of 37 eyewitness experts agreed with this statement (Marr et al., 2020). Methodological differences in the stimuli, induction of stress, stressor verification, stressor timing, and retrieval timing may be the key reasons in explaining these contrasting findings (see Sauerland et al., 2016).

The to-be-remembered stimuli often differ between the fields. First, some evidence suggests that stress effects on memory are stronger for emotional stimuli than neutral stimuli (e.g., Cahill et al., 2003; Smeets et al., 2008), perhaps due to the amygdala's sensitivity to both adrenergic and glucocorticoid stress responses (Joëls et al., 2011; 
McGaugh, 2015). However, a meta-analytic review found no evidence that valence was a moderator of acute stress at encoding on memory performance (Shields et al., 2017). Second, the eyewitness field has more commonly examined encoding stress effects on eyewitness identification or face recognition performance. Such research has often found negative effects of encoding stress on face recognition performance (e.g., Davis et al., 2019; Deffenbacher et al., 2004; Morgan et al., 2004; Pezdek et al., 2020). However, in an eyewitness study conducted by Sauerland et al. (2016), participants were stressed using the Maastricht Acute Stress Test (MAST; Smeets et al., 2012) before they witnessed a live theft. Levels of acute stress were confirmed by cortisol measurements. However, this acute stress had no impact on identification performance in a lineup viewed 6-8 days later.

In the fundamental memory field, encoding enhancements have mainly been found with non-facial stimuli such as words, pictures, and slideshows (Domes et al., 2002; Henckens et al., 2009; Payne et al., 2006). Differences in type of to-be-remembered stimuli may play a role in the contrasting findings, as some research suggests that faces are processed differently than other forms of stimuli (e.g., Diamond \& Carey, 1986; Kanwisher \& Yovel, 2006; Sato \& Yoshikawa, 2013; Woodhead \& Baddely, 1981). Several theories suggest that we may learn and recognize faces differently than we do other types of stimuli. For example, the face-specificity hypothesis suggests that faces are distinctively processed in unique areas of the brain (i.e., the fusiform face area) than other stimuli (Kanwisher \& Yovel, 2006), and the expertise hypothesis proposes that humans are generally experts in face recognition (e.g., Diamond \& Carey, 1986). Thus, although there is some evidence to suggest that stress during encoding enhances memory for stimuli like words, static pictures, and slideshows (e.g., Domes et al., 2002; Henckens et al., 2009; Payne et al., 2006; Zoladz et al., 2011; but see Schwabe \& Wolf, 2010; Zoladz et al., 2014), it is possible that stress does not affect memory for faces to the same extent. Some experimental results support this idea. For instance, Paul et al. (2016) found that acute stress experienced before a visual discrimination task impaired spatial information but did not affect the discrimination of faces. Wiemers et al. (2013), using the Trier Social Stress Test (Kirschbaum et al., 1993), found that stressed participants outperformed nonstressed participants in face and central object recognition, but not peripheral object recognition. These differences in stimuli may explain at least some of the discrepancies between the eyewitness research and fundamental memory research as fundamental memory experiments most often use words or other non-facial images as stimuli.

Many eyewitness experiments use stressors such as violent videos (Clifford \& Hollin, 
1981; Cutler et al., 1987; Kramer et al., 1990), electric shocks (Brigham et al., 1983; Tooley et al., 1987), threats of injection (e.g., Maass \& Kohnken, 1989; Peters, 1988), and high-fidelity interactive training scenarios (Hope et al., 2016). These stress inductions are typically verified with self-report measures (e.g., Buckhout et al., 1974; Davis et al., 2019). Other experiments have grouped participants using self-reports about trait stress, state stress, or test anxiety (Bailis \& Mueller, 1981; Mueller et al., 1979; Nowicki et al., 1979) and drawn conclusions about stress and memory based on group differences. Such varied methods may elicit different levels of arousal and stress. However, it is difficult to evaluate the efficacy of stress inductions based on self-report data alone, as subjective self-reports of stress do not always correspond to physiological stress responses (Hellhammer \& Schubert, 2012). Additionally, methods that increase arousal, thus initiating a noradrenergic response, do not necessarily produce the increases in cortisol that are associated with a physiological stress response (Dickerson \& Kemeny, 2004). In contrast, researchers in the fundamental memory field typically use validated laboratory stressors to induce stress (Kirschbaum et al., 1993; Schwabe et al., 2008a; Smeets et al., 2012) with physiological measures serving to verify the stress induction in addition to subjective measures.

Differences in timing of the stress induction and memory encoding may also partly account for the contrasting findings. Stress modulates memory formation and retrieval in a timedependent manner, closely linked to the temporal action profile of major stress mediators in the brain, in particular noradrenaline and glucocorticoids (Hermans et al., 2014; Jöels et al., 2011; Quaedflieg \& Schwabe, 2018; Schwabe et al., 2012). Eyewitness studies mostly examine stress during encoding (e.g., most experiments in the Deffenbacher et al. meta-analysis, 2004; Hulse \& Memon, 2006; Morgan et al., 2004). Examining this acute stress stage reflects the reality of an eyewitness experience during a crime. In contrast, much of the fundamental memory research conducted on stress during the encoding phase focuses on the delayed stage, that is, when participants encoding information after engaging in a stressor (e.g., Wolf, 2012, Exp 2; Zoladz et al., 2011; Quaedflieg et al., 2013), as cortisol peaks about 15-20 minutes following the stress induction.

The two fields also vary in timing of the encoding and retrieval phases. Many eyewitness experiments examining stress and memory conduct the encoding and retrieval sessions on one day (e.g., Davis et al., 2019; Hulse \& Memon, 2006; Valentine \& Mesout, 2008). However, a separation between encoding and retrieval is necessary to distinguish stress effects during encoding or consolidation from stress effects during retrieval on memory. 
If encoding and retrieval take place in one day, then it is impossible to say what memory phase was influenced by the stress. A solution to this issue is to separate encoding and retrieval by at least 24-hours, as commonly done in fundamental memory studies (e.g., Shermohammed et al., 2019; Vogel \& Schwabe, 2016; Wolf, 2012, Exp 2; Zoladz et al., 2011, 2014).

To briefly summarize, the fundamental field generally shows that acute encoding stress enhances memory performance (e.g., Henckens et al., 2009; Shields et al., 2017; Vogel \& Schwabe, 2016), whereas the eyewitness field suggests that acute stress impairs memory performance (e.g., Davis et al., 2019; Deffenbacher et al., 2004; Morgan et al., 2004). Variations in methodology likely contribute to the discrepant findings regarding the effects of acute stress on memory performance in the eyewitness and fundamental memory fields. As such, when designing studies and interpreting results, it is imperative to consider potential moderators including type of stimuli, type of stress induction and manipulation check, stressor and retrieval timing.

\section{Effects of Acute Stress at Retrieval on Memory Performance}

Stress experienced just prior to retrieval often impairs memory performance (e.g., Quaedflieg \& Schwabe, 2018; Shields et al., 2017; Wolf, 2017). This impairment is greatest during the cortisol peak caused by non-genomic actions of glucocorticoids that develop about 15-20 minutes following the stress induction (de Quervain et al., 1998; Joëls \& Baram, 2009; Joëls et al., 2011). Impairments continue as the delayed genomic effects develop, around 60-90 minutes post-stressor and last for hours (Schwabe \& Wolf, 2014; Shields et al., 2017; Wolf, 2017). Accordingly, the specific timing of the stressor is crucial. When the memory test takes place about 20-30 min post-stressor, that is, during the delayed stage (i.e., when stress-induced cortisol increases), results often show memory impairments (Schönfeld et al., 2014; Schwabe \& Wolf, 2014). When the memory test takes place during the acute stress stage (i.e., just after or during the stress induction and therefore before the stress-induced cortisol peak), such impairments do not regularly occur (e.g., Schwabe \& Wolf, 2014). In fact, one study reported that retrieval performance during stress was positively associated with the noradrenergic stress response (Schönfeld et al., 2014).

Apart from stressor timing, the type of memory test and valence play a role in the impairing effects of retrieval stress. Stress prior to retrieval impairs both recall (e.g., Kuhlmann et al., 2005; Schönfeld et al., 2014; Smeets et al., 2008) and recognition performance (Li et al., 2013; Schwabe \& Wolf, 2014), with stronger effects for recall than recognition (see 
Gagnon \& Wagner, 2016). Furthermore, retrieval stress tends to elicit larger negative effects for emotional than neutral stimuli (e.g., Kuhlmann et al., 2005; Schönfeld et al., 2014). Thus, the common view is that stress interferes with retrieval performance, though stressor timing, type of memory test, and valence moderate these effects.

Few experiments have investigated the effects of retrieval stress on identification accuracy or face recognition, and findings are mixed. Li et al. (2013) found that stress inducted immediately prior to retrieval impaired face recognition sensitivity. However, in a subsequent study the same authors found no statistically significant effects of stress on face recognition accuracy ( $\mathrm{Li}$ et al., 2014). As far as I am aware, no research has yet examined effects of stress during retrieval on face recognition memory. Understanding how experiencing stress during retrieval is likely relevant for applied legal contexts, where witnesses may be stressed when attempting to identify a suspect in a police lineup.

\section{The Present Experiment}

Combining methodology from the eyewitness and fundamental memory fields, the aim of the current two experiments was to examine the effects of acute stress at encoding and retrieval on face and word recognition performance. I induced stress by means of the MAST (Smeets et al., 2012), used physiological measures to verify the stress induction, and inserted a 24-26 hour interval between encoding and retrieval. I also tested the effect of stress stage by inserting encoding and retrieval stages both during the stress induction (i.e., acute stress stage, when noradrenergic activity ensues) and after the stress induction (i.e., delayed stage, when stress-induced cortisol peaks).

In Experiment 2, I examined four between-subjects groups: no stress, stress at encoding, stress at retrieval, and stress at both encoding and retrieval and examined stress stage (acute stress vs. delayed) as a within-subjects measure. Based on models and past findings from the fundamental memory field (e.g., Diamond et al., 2007; Hoscheidt et al., 2014; Joëls et al., 2006; Vogel \& Schwabe, 2016; Wiemers et al., 2013), I predicted that participants who experienced stress during encoding (but not retrieval) would show enhanced face recognition memory compared to the other groups (Hypothesis 1). I also predicted that those who experienced stress during retrieval (but not encoding) would perform the poorest on the face recognition memory task out of the four groups (Hypothesis 2). Furthermore, I expected that the predicted stress effects would be larger during the cortisol peak (i.e., $15 \mathrm{~min}$ post-stressor; delayed stage) than before cortisol had peaked (i.e., during stressor; acute stress stage, Hypothesis 3). 
Following null results in Experiment 2, Experiment 3 compared participants who experienced encoding stress with those who did not to examine memory performance for face versus word stimuli. I predicted that participants stressed during encoding would show better recognition memory for words than non-stressed participants (Hypothesis 4). Based on my results from Experiment 2 and extant literature concerning the distinctiveness of face processing (e.g., McGugin et al., 2014; Kanwisher \& Yovel, 2006), I expected to obtain similar findings for faces as those in Experiment 2. That is, I hypothesized no statistically significant difference in memory performance for faces between stress conditions (Hypothesis 5). I also examined the role of stress stage (acute stress vs. delayed) though I had no a priori hypotheses for these variables due to the null findings in Experiment 2.

\section{Method}

Both experiments were preregistered on the Open Science Framework (Experiment 2: https://osf.io/k8x5q/?view_only=563a7d16459a47a7aebff54ccd70bf09; Experiment 3: https://osf.io/sqxgb/?view_only=b0564519ca604a17a65b1a34488af187).

\section{Participants}

Based on a priori power analyses conducted using G*Power (Faul et al., 2007) for an Analysis of Variance (ANOVA) for fixed effects, special, main effects and interactions with $80 \%$ power, $\otimes=.05$, and a medium-large effect size $\left(f=.26-30^{4}\right.$; based on past relevant work including Shields et al., 2017), the target sample size for both experiments was $N=119$. I used one effect size across hypotheses focusing on the main independent variable of stress, as past research has often conflated the time periods that I distinguished between (i.e., acute vs. delayed stress stage; see Shields et al., 2017), though I predicted that stress effects may be larger during the delayed period (i.e., Hypothesis 3).

I recruited participants between the ages of 18 and 35 from the university and local community using posters, handouts/flyers, social media, and lecture visits. Consistent with relevant previous research examining factors that may affect physiological stress reactivity (e.g., Shields, 2020; Strahler et al., 2017), I screened for and excluded participants who habitually smoked ( $>5$ cigarettes per day), drank alcohol ( $>15$ drinks per week), or

\footnotetext{
${ }^{4}$ For Experiment 2, I preregistered that I aimed for a small to medium effect size (between $f=.22$ and .26 ). Due to difficulties recruiting eligible participants and a slight change in the analysis plan (as described later), I tested enough participants to provide statistical power for the medium-large effect size of $f=.30$, as indicated here.
} 
used drugs (more than once per month). Participants were also excluded for a variety of other health reasons (i.e., BMI $<17$ and $>30$, use of medication containing cortisol, recent vaccinations, psychological treatments, cardiovascular problems, or endocrine disorders). Because sex hormones can affect cortisol reactivity (e.g., Kirschbaum et al., 1999; Kudielka et al., 2009; Strahler et al., 2017), females who were not taking a form of hormonal birth control were excluded in order to keep the sample homogeneous. Only white participants were included to avoid influence of the own-race effect on face identification (e.g., Meissner \& Brigham, 2001). Additional exclusion criteria for Experiment 3 included participation in Experiment 2 or in another stress-based laboratory study using the MAST within the past month, as past research shows that the MAST can be used repeatedly with no significant signs of habituation or sensitization with intervals of three weeks and one month (Quaedflieg et al., 2017).

For Experiment 2, 144 participants were tested. Ten participants withdrew during or after Day 1, three experienced a computer program malfunction, and 11 participants indicated having previously seen one or more of the face stimuli and were excluded post-testing. The final sample $(N=120)$ included 41 men and 79 women (age range 18-31 years; $M$ $=22.04, S D=2.87)$. The majority were university students $(91.70 \%)$ and the rest were not students $(8.30 \%)$. Most of the students were completing their Bachelors degrees $(75.50 \%)$ and the rest were completing a graduate degree $(24.50 \%)$.

For Experiment 3, 137 participants were tested. Seven participants withdrew on or after Day 1, three experienced a computer program malfunction, and six participants indicated having previously seen one or more of the face stimuli and were excluded post-testing. The final sample $(N=121)$ included 34 men and 87 women (age range 18-34 years; $M=$ $22.21, S D=2.94)$. The majority were university students $(94.2 \%)$ and the rest were not students (5.80\%). Again, most of these students were completing their Bachelors degrees (60.50\%), with the rest completing a graduate degree (39.50\%).

Participants were asked to follow several rules before Day 1 (Experiments 2 and 3) and Day 2 (Experiment 2). These instructions included not drinking alcohol the night before, getting a full night of sleep, and refraining from eating, drinking anything besides still water, exercising, smoking, or brushing teeth for at least two hours prior to the session. Participants received either course credit or $€ 20$ (Experiment 2) or $€ 15$ (Experiment 3) in gift vouchers on completion. This study was approved by the ethical committee of the Faculty of Psychology and Neuroscience at Maastricht University. 


\section{Design}

In Experiment 2, I examined four between-subjects groups (condition: encoding stress vs. retrieval stress vs. stress at encoding and retrieval vs. no stress) and examined stress stage as a within-subjects factor (stress stage: acute stress vs. delayed). Experiment 3 had a 2 (condition: no encoding stress vs. encoding stress) x 2 (stress stage: acute vs. delayed) x 2 (stimulus type: faces vs. words) mixed design. Stress stage and stimulus type were withinsubjects factors. For both experiments, participants were semi-randomly assigned to the stress conditions, balancing gender across groups. The dependent variables included overall accuracy, proportion of hits, proportion of false alarms, sensitivity ( $d$ '; exploratory for Experiment 2) and response bias indices ( $c$; exploratory for Experiments 2 and 3). Sensitivity was calculated by the difference between the z-scores of number of hits and number of false alarms, where the larger $d$ ', the better one's performance. Response bias was calculated by the sum of the z-scores of hits and false alarms divided by two, where 0 represents no bias, values less than 0 represent a liberal bias, and values greater than 0 represent a conservative bias. ${ }^{5}$ Dependent measures for the manipulation check included negative affect scores, diastolic and systolic blood pressure measurements, and salivary cortisol levels (Experiment 3).

\section{Materials}

\section{Stimuli and Memory Test}

Faces (Experiments 2 and 3). Images were taken from an image database comprised of past and current student and staff volunteers at the Maastricht University. In Experiment 2, I used two color photographs each of 12 males and 12 females as targets (i.e., 48 photographs in total). Targets were all white young adults with a variety of hair colors, hair lengths, hair textures, facial shapes, body types, and eye colors. Photographs of each individual were taken on the same day with no changes in appearance (e.g., haircuts) besides facial expression. Participants viewed two distinct images of each target at encoding, once as a smiling portrait picture and once as a full body picture. In Experiment 3 , only the smiling portrait picture was shown (i.e., 24 pictures total). Each image was displayed for $4 \mathrm{~s}$ with a 1-s interstimulus interval which allowed for the images to fit within the time-constrained blocks in the (control) MAST procedure. In order to assess

\footnotetext{
${ }^{5}$ When proportions of hits or false alarms equaled $0 \%$ or $100 \%$, adjusted rates were used for the $d$ ' and $c$ calculations. When $0 \%$, hit or false alarm rates were calculated as $0.5-n$; When $100 \%$, hit or false alarm rates were calculated as, $(n-0.5) / n$, where $n=$ number of trials.
} 
the within-subjects factor of stress stage, the same individuals needed to be presented within the same blocks (i.e., acute stress stage vs. delayed stage) at encoding and retrieval. The best way I identified to counterbalance these blocks in a logical way was to split the faces into the two distinguishable groups of gender. Thus, half of the images (either all males or females) were shown at the acute stress stage and the remaining images were shown at the delayed stage. I counterbalanced gender order and randomized the order of the images in blocks. The recognition test comprised 48 color photographs ( 24 old, 24 new; 24 male, 24 female) of previously unseen neutral portrait pictures and consisted of a yes/no identification question. I used previously unseen photos at test to ensure that the task measured face recognition as opposed to image recognition (see Burton, 2013). The total number of faces used is comparable to other recent similar work using face recognition tasks (e.g., Davis et al., 2019; Pezdek et al., 2020). Participants provided confidence judgements for all responses on a scale of $0-100 \%$ using a sliding bar. ${ }^{6}$

Words (Experiment 3). Twenty-four negatively valenced words served as targets. Commonly-known negatively valenced nouns were chosen from the Affective Norms for English Words (ANEW; Bradley \& Lang, 1999). I used negative words because some research suggests stronger stress effects on memory for emotional stimuli (e.g., Cahill et al., 2003; Joëls et al., 2011; Smeets et al., 2008, but see Shields et al., 2017). Groups of words were balanced for valence, arousal, frequency, and length. The presentation of and retrieval test for words was analogous to the procedure for faces, again with 24 targets and 24 fillers. The order of recognition tests for faces and words were counterbalanced.

\section{Maastricht Acute Stress Test (MAST)}

The MAST (Smeets et al., 2012) is a validated laboratory stressor. In this task, participants engage in blocks of hand immersion into ice-cold water $\left(2-4^{\circ} \mathrm{C}\right)$, combined with blocks of socially-evaluated mental arithmetic in front of a critical experimenter who gives negative feedback throughout the task Additionally, participants consent to and are told they are being video recorded for later facial expression analysis. Thus, a second monitor displays a live video of the participant's face to further induce stress, though in reality no recordings are taken. Participants in the no stress conditions were exposed to the control version of this task, which includes hand immersion into room-temperature water $\left(35^{\circ} \mathrm{C}\right)$, basic counting from 1 to 25 , and no mention of a video recording. The traditional MAST was slightly varied in these experiments. That is, blocks of stimuli (Experiment 2: faces;

${ }^{6}$ Confidence data were not analyzed or reported in this paper. 
Experiment 3: faces and words) were intermixed with the blocks of hand immersion and mental arithmetic. There were four $30 \mathrm{~s}$ blocks during the acute stress stage and four $30 \mathrm{~s}$ blocks during the delayed stage. This version of the MAST took $16.5 \mathrm{~min}$ in total. Figure 3.1 depicts a detailed timeline of the procedure.

\section{Affect}

The Positive and Negative Affect Schedule (PANAS; Watson et al., 1988) consists of two mood scales containing 10 items that measure positive and negative affect. For each item (e.g., interested, excited, nervous, distressed, etc.), participants indicate to what extent they feel that way at the present moment on a 5-point Likert scale (from very slightly or not at all to extremely). Affect was measured at three timepoints as shown in Figure 3.1: during the baseline questionnaires, after the MAST, and at the end of the session after the delayed stage.

\section{Blood Pressure}

To examine autonomic nervous system activation, I collected systolic and diastolic blood pressure using an Omron Blood Pressure Monitor 705IT (Coleman et al., 2006). Blood pressure was measured eight times throughout the procedure: once during the first saliva sample, once after the MAST anticipation block, twice during the MAST, once just after the MAST, once during the second saliva sample, and twice during the delayed stage (see Figure 3.1). In Experiment 3, the original blood pressure monitor failed, so an updated version (Omron M7 IT HEM-7322T-E) was used after 11 participants had been tested, although nothing changed procedurally.

\section{Cortisol}

I collected saliva samples from participants twice on Day 1 (Experiment 2 and 3) and Day 2 (Experiment 2) with synthetic Salivette (Sarstedt, Etten-Leur, the Netherlands) devices. In the absence of funding for sample processing, the samples from Experiment 2 have not been analyzed. The saliva samples from Experiment 3 were frozen and stored at $-20{ }^{\circ} \mathrm{C}$ until analysis. After thawing, Salivettes were centrifuged at 3,000 rpm for $5 \mathrm{~min}$, which resulted in a clear supernatant of low viscosity. Salivary concentrations were measured using commercially available chemiluminescence immunoassay with high sensitivity (IBL International, Hamburg, Germany). The intra- and inter-assay coefficients for cortisol were both below $9 \%$. 


\section{Figure 3.1}

Timeline for Experiment 2 (Day 1 and 2) and Experiment 3 (Day 1)
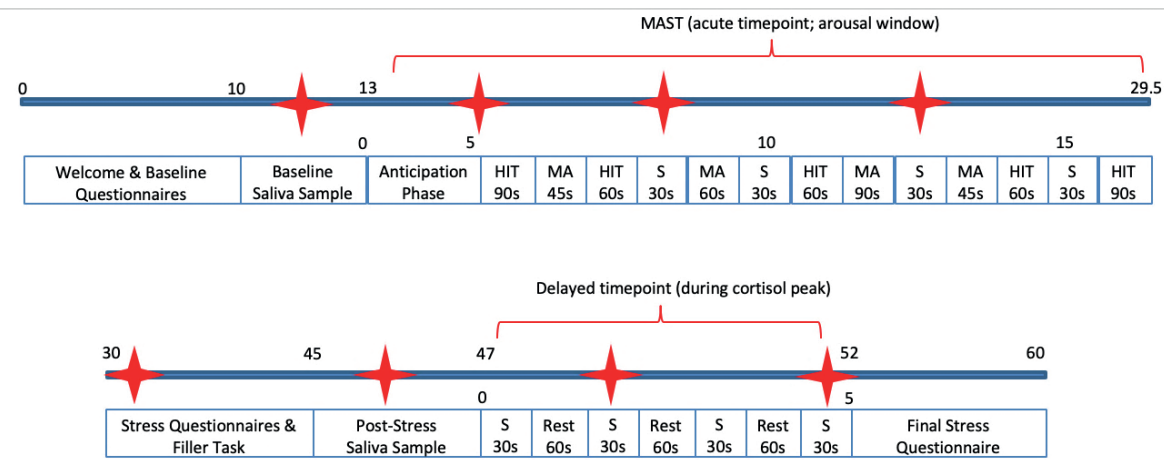

Note. HIT $=$ hand immersion trial. $\mathrm{MA}=$ mental arithmetic. $S=$ encoding (Day 1) or recognition (Day 2) of stimuli. Day 2 of Experiment 2 not visually depicted.

$+=$ blood pressure measurement. MAST $=$ Maastricht Acute Stress Test or control version.

\section{Procedure}

I tested participants in both experiments between 12:00 pm and 6:00 pm to account for diurnal cortisol levels (Shields, 2020). After giving consent, participants completed the PANAS and demographic questions as well as other filler questionnaires unrelated to this study. Participants were aware that they would be asked to recognize the stimuli during the follow-up session. After $10 \mathrm{~min}$, participants provided a saliva sample and engaged in the (control) MAST, while viewing blocks of faces (Experiment 2) or blocks of faces or words (Experiment 3). This constituted the acute stress stage of face encoding. Next, participants again completed the PANAS before participating in a filler task (Tetris). Once $15 \mathrm{~min}$ had passed since the end of the (control) MAST (to await the expected cortisol response; Smeets et al., 2012), participants provided another saliva sample and completed the delayed stage of face encoding, consisting of blocks of faces (Experiment 2) or faces and words (Experiment 3 ) in 30 s chunks which mimicked encoding at the acute stress stage. Following this, participants once again completed the PANAS. The Day 1 session took 1 hour to complete.

Participants returned to the lab 24-26 hours later. For Experiment 2, the procedure on Day 2 was identical to Day 1 with the exception that instead of encoding faces during and after the (control) MAST participants took part in a face recognition test at these times. For Experiment 3, the Day 2 session did not involve engagement with the (control) 
MAST but instead consisted only of a recognition test which took 15 min to complete. Following the recognition test, participants received a debriefing and compensation. Figure 3.1 shows an overview of the procedure timeline.

\section{Data Analysis}

To verify the stress manipulations, I conducted mixed ANOVAs on the effect of stress condition (i.e., stress vs. no stress) on self-reported negative affect scores and systolic and diastolic blood pressure across each testing day (i.e., timing: three levels for negative affect; eight levels for blood pressure). For Experiment 3, I also conducted a mixed ANOVA on the effect of stress condition on salivary cortisol pre- and post-stressor. The main analyses for Experiment 2 differed to those that were pre-registered. These deviations were decided upon prior to analyzing the data. Specifically, rather than conducting two separate 2 (stress at encoding: yes vs. no) x 2 (stress at retrieval: yes vs. no) for each testing stage, I conducted a mixed ANOVA with condition (no stress vs. encoding stress vs. retrieval stress vs. both) as between subjects and stress stage (acute stress vs. delayed) as within subjects factor. I made this decision in order to compare the stages as past research has emphasized that stress effects on memory are time sensitive (e.g., Quaedflieg \& Schwabe, 2018; Schwabe et al., 2012). Distinctly examining the four stress conditions in one analysis also allowed for a clearer test of the hypotheses, which placed emphasis on the encoding only and the retrieval only groups. Thus, for both experiments, I conducted one-way mixed ANOVAs to analyze the effects of condition on overall accuracy, proportion of hits, proportion of false alarms, sensitivity, and response bias. For all tests, when Mauchly's test indicated that the assumption of sphericity was violated, I corrected the degrees of freedom using Greenhouse-Geisser estimates of sphericity.

\section{Results}

\section{Manipulation Checks}

Table 3.1 provides an overview of the main inferential statistics for the manipulation checks across both experiments.

\section{Negative Affect}

Higher scores on the negative affect portion of the PANAS reflect higher self-reported negative affect. In Experiment 2 on Day 2, negative affect scores were missing for one participant $(N=119)$. Across both experiments, stress differentially affected negative 
affect scores depending on the timing (see Table 3.1). The pattern of results was similar in each analysis, with follow-up tests revealing no statistically significant differences between groups at baseline (all $p s \geq .050$, see Table 3.1). However, right after the stressor (i.e., MAST), stressed participants reported statistically significantly higher levels of negative affect than non-stressed participants (all $p s \leq .001$, see Table 3.1). Thus, negative affect scores in both experiments subjectively confirm the stress induction. Figure 3.2 displays changes in negative affect across time in both experiments.

\section{Blood Pressure}

In Experiment 3, blood pressure measurements were missing for one participant $(N=$ 120). In both experiments, stress differentially affected both systolic and diastolic blood pressure depending on the timing (see Table 3.1). Follow-up tests revealed no statistically significant differences between stress conditions at baseline (all $p s \geq .419$, see Table 3.1). However, as expected, during the stressor (i.e., MAST 1), the stress groups showed statistically significantly higher blood pressure than the non-stressed group (all $p s \leq .003$, see Table 3.1). Thus, blood pressure measures in both experiments confirm physiological arousal. Figure 3.3 shows changes in blood pressure measurements across time on each testing day.

\section{Salivary Cortisol (Experiment 3)}

Stress differentially affected salivary cortisol levels as a function of timing (see Table 3.1). Follow-up tests revealed no statistically significant differences in salivary cortisol between stress conditions at baseline ( $p=.400$, see Table 3.1). As expected, after the stressor, the stress group showed statistically significantly higher salivary cortisol levels than the non-stressed group, $(p<.001$, see Table 3.1). Thus, the salivary cortisol levels confirm the acute stress induction. Figure 3.4 shows changes in salivary cortisol levels before and after the stressor. I also exploratorily grouped cortisol responders vs. non-responders, with $62.30 \%$ of the stress group showing a high cortisol response and the other $37.70 \%$ showing a low cortisol response $(1.5 \mathrm{nmol} / \mathrm{l}$ increase, Miller et al., 2013). However, this exploratory analysis examining high responders, low responders, and participants who were not stressed revealed no statistically significant differences of acute stress on any memory measure (all $p$ s $>.050$; see Table B.A in Appendix B), and thus, no further analyses on these subgroups were performed or reported. 


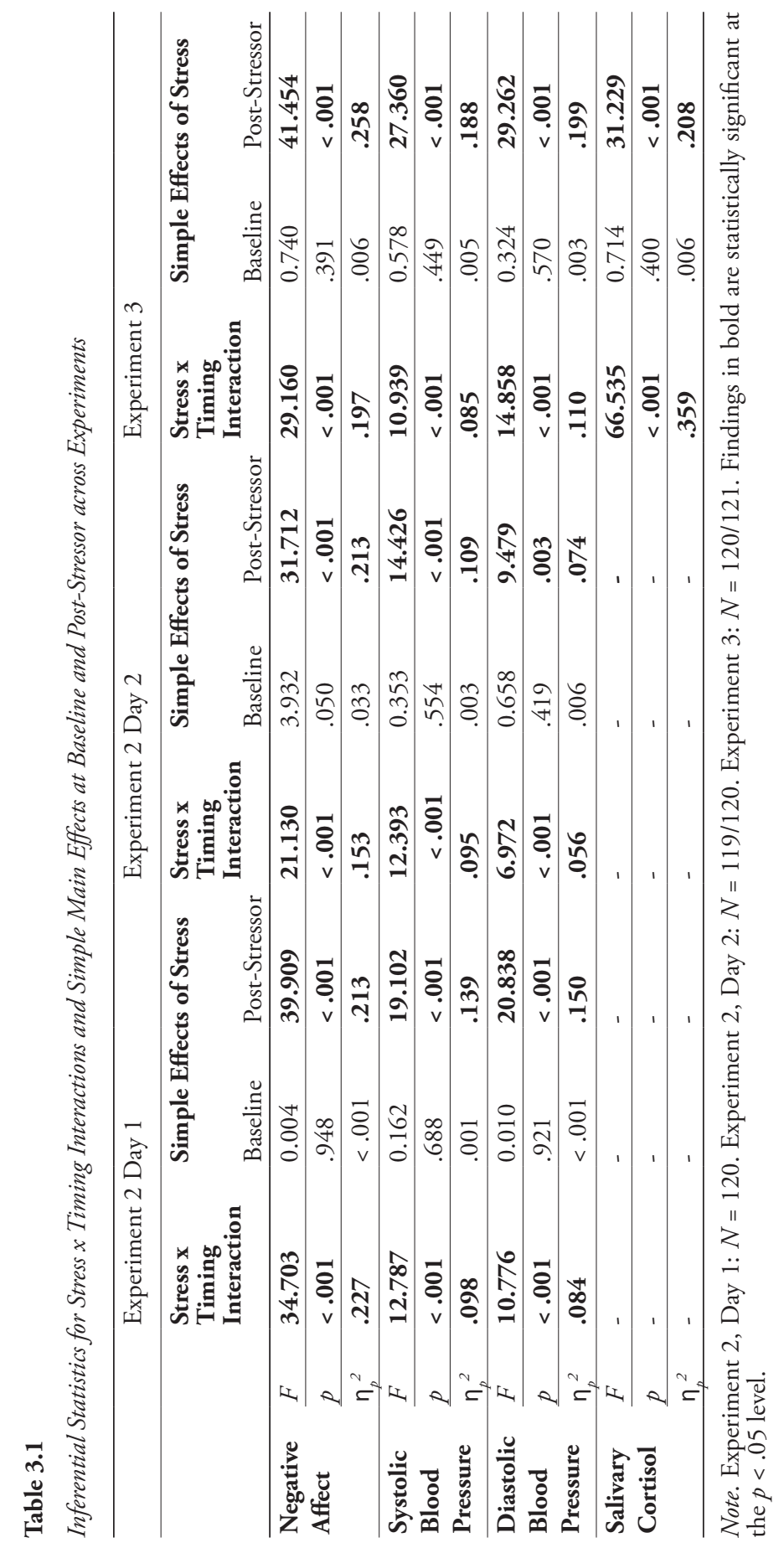




\section{Figure 3.2}

Negative Affect Scores over Time in Experiments 2 and 3 Across Stress Conditions

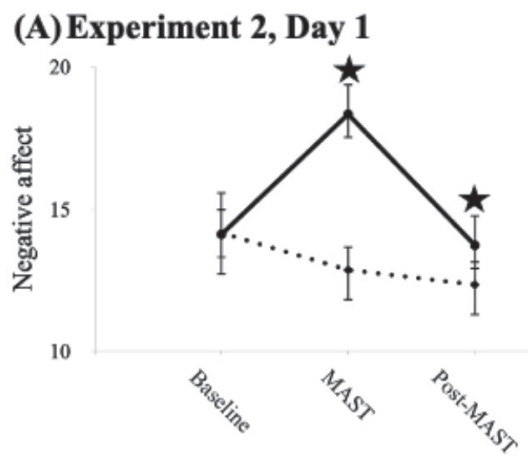

\section{(B) Experiment 2, Day 2}

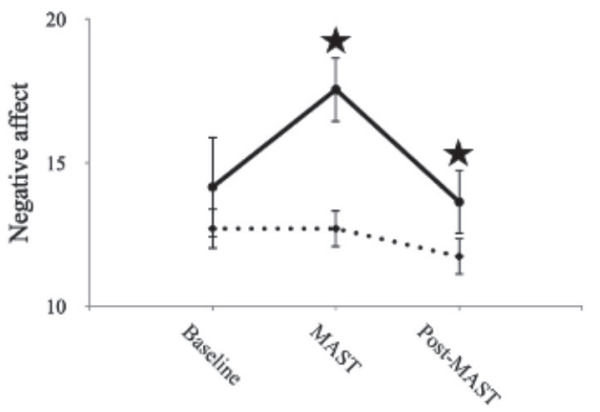

\section{(C) Experiment 3}

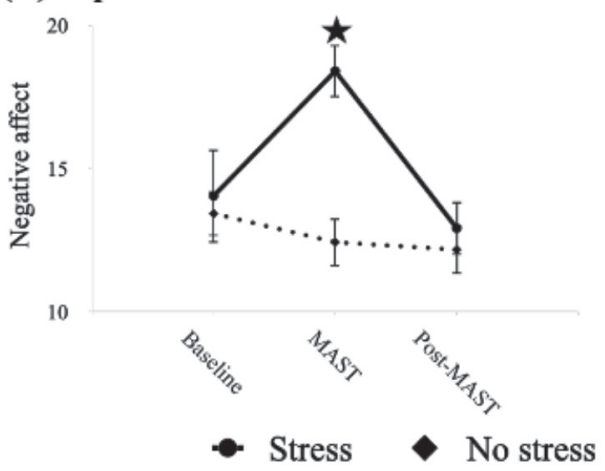

Note. Possible negative affect scores range between 10 and 50 . Negative affect scores are from the Positive and Negative Affect Schedule. MAST = Maastricht Acute Stress Test. Error bars $=95 \%$ confidence intervals. $\star=p<.05$. 


\section{Figure 3.3}

Systolic and Diastolic Blood Pressure over Time in Experiments 2 and 3 Across Stress Conditions
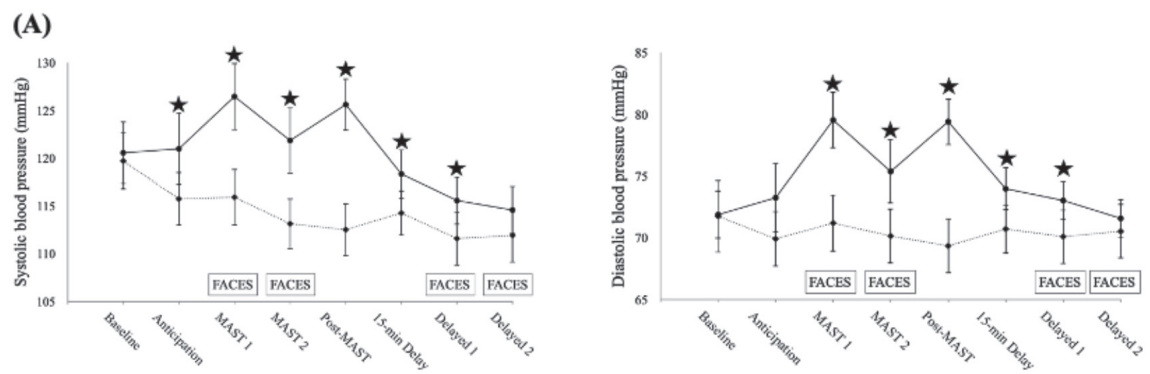

(B)
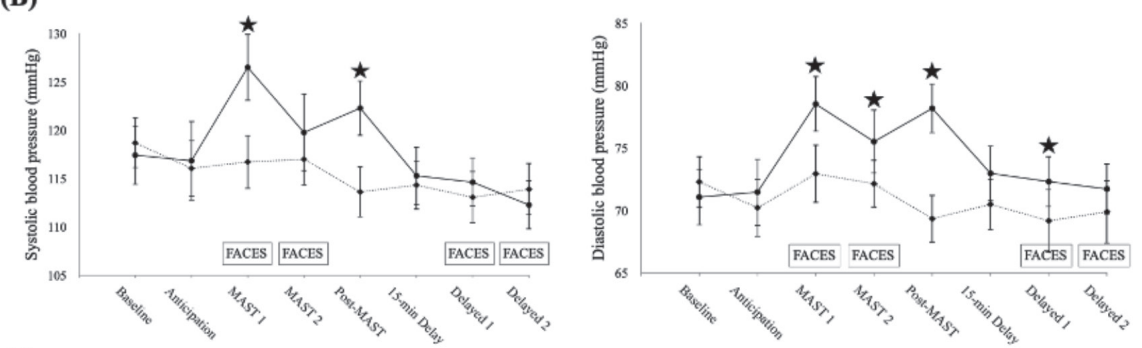

(C)
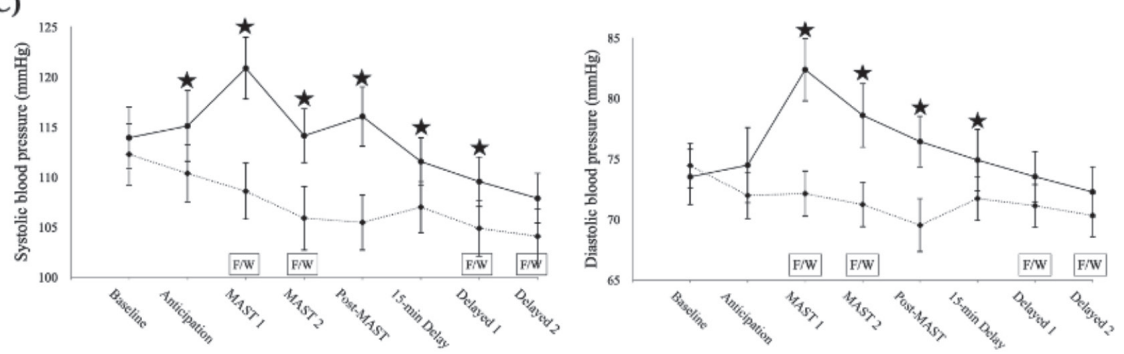

- Stress

No stress

Note $(\mathrm{A})=$ Experiment 2 , Day 1. $(\mathrm{B})=$ Experiment 2 , Day 2. $(\mathrm{C})=$ Experiment 3 , Day $1 . \mathrm{mmHg}$ $=$ millimeters of mercury. MAST $=$ Maastricht Acute Stress Test (acute stress stage). Delayed $=$ delayed stage. Faces and $\mathrm{F} / \mathrm{W}=$ times when faces (and words) were encoded or recognized. Error bars $=95 \%$ confidence intervals. $\star=p<.05$. 


\section{Figure 3.4}

Salivary Cortisol Level Pre-and Post-MAST in Experiment 3 Across Stress Conditions

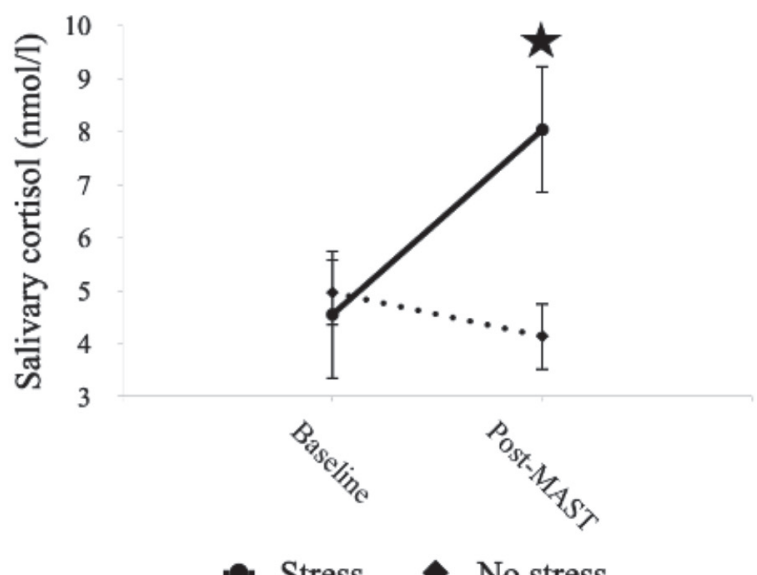

Note. MAST $=$ Maastricht Acute Stress Test. Error bars $=95 \%$ confidence intervals. $\downarrow$ \ $=p<.05$.

\section{Effects of Acute Stress on Memory Performance}

Tables 3.2-3.4 provide a summary of the inferential and descriptive statistics for all dependent variables regarding the main analyses from each experiment.

\section{Experiment 2}

For face recognition, I found no statistically significant effects of condition, stress stage, or interactions between these factors on overall accuracy, proportion of hits, or proportion of false alarms $s^{7}, 8$. Thus, Hypotheses 1, 2, and 3 were not supported. I also examined sensitivity $(d)$ and response bias $(c)$ as exploratory (i.e., non-preregistered) outcome variables. There were no statistically significant effects for sensitivity, $p s \geq .060$. For response bias, the main effect of stress stage was statistically significant. Figure 3.5 illustrates that responding was more liberal at the acute stress stage than at the delayed stage although participants in general were liberal rather than conservative in their responding.

\footnotetext{
${ }^{7}$ As preregistered, I also conducted the analyses after removing outliers (scores $\geq 2.5$ SDs from the mean; see Table B.B in Appendix B). These analyses ( $\left.N_{s}=112 / 118\right)$ returned analogous patterns of results.

${ }^{8}$ Additionally, I analyzed data with guesses (confidence at $50 \%$ or below) removed (see Table B.C in Appendix B). No statistically significant interaction or main effects emerged for any of the outcome variables. Thus, the main effect of stress stage on response bias was no longer statistically significant although responding was still in general liberal rather than conservative.
} 
Figure 3.5

Response Bias (c) Across Stress Conditions for Acute Stress and Delayed Stages in Experiment 2

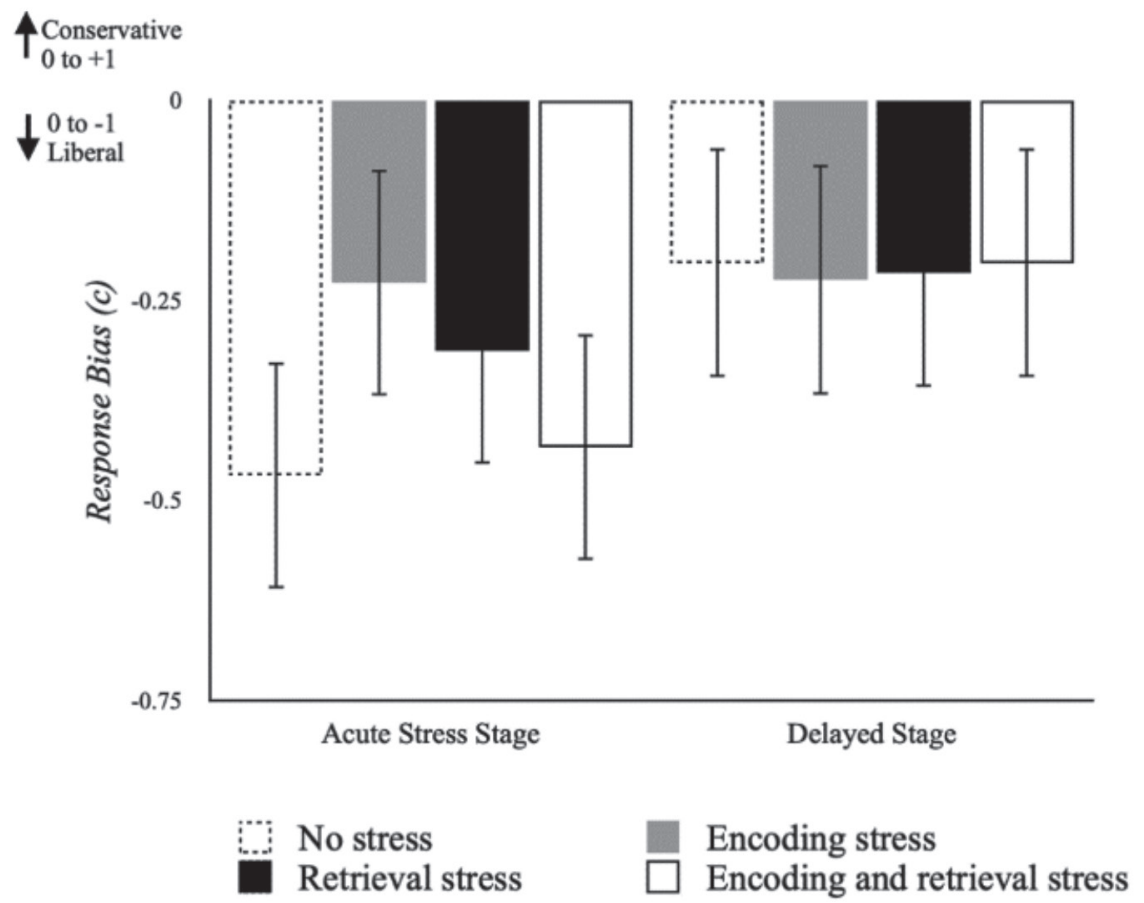

Note. Error bars $=95 \%$ confidence intervals.

I also examined the data using Bayesian ANOVAs with JASP version 0.13.1 (JASP Team, 2020). I adopted a weakly informative prior by setting the $r$ scale fixed effect at a default value of 0.5 . Results were similar to those reported above, with only the evidence regarding response bias (c) pointing away from the null hypothesis. Specifically, for the main effect of stress stage on response bias, there was very strong evidence for the alternative hypothesis $\left(\mathrm{BF}_{10}=37.037\right.$; Jarosz \& Wiley, 2014; Jeffreys, 1961; Raftery, 1995). All other estimated BFs examining main and interaction effects of the dependent variables suggested the data were in favor of the null hypothesis, with evidence ranging from anecdotal to strong (i.e., from $\mathrm{BF}_{01}=1.340$ to $\mathrm{BF}_{01}=13.356$, see Table B.D in Appendix B). 


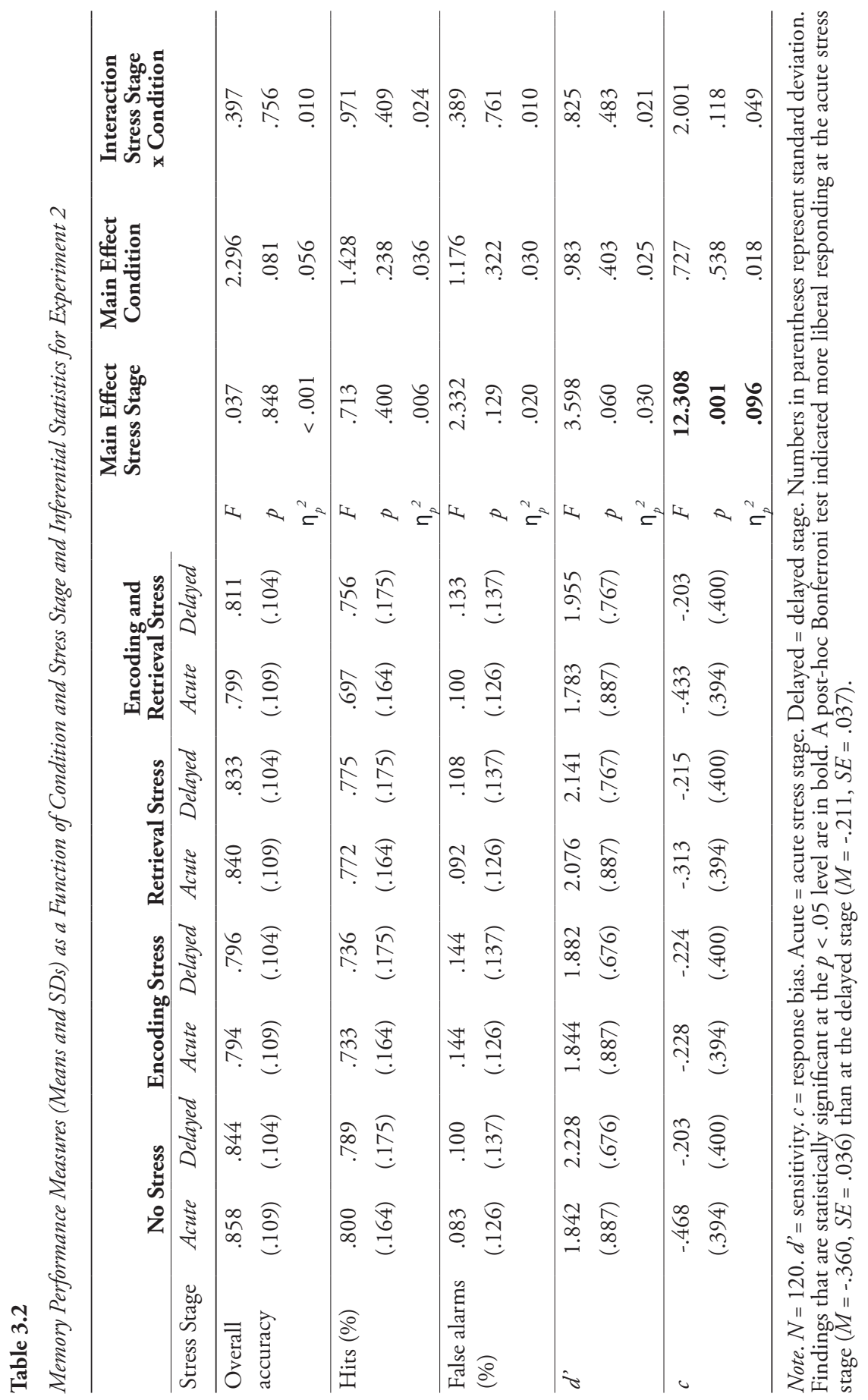




\section{Experiment 3}

Memory measure data were missing for one participant, and thus they were excluded from the following analyses $(N=120)$. Failing to support Hypothesis 4, I found no evidence for effects of acute stress on word recognition performance. However, supporting Hypothesis 5, I again found no statistically significant evidence that acute stress affected face recognition memory. The main effect of condition was non-significant for all dependent measures. A main effect of stimulus type indicated a higher false alarm rate for words than faces. Statistically significant interactions between stimulus type and stress stage on hit rate and response bias modified main effects of stimulus type. Specifically, words elicited a higher hit rate and a more conservative response bias than faces at both the acute stress stage, hits: $F=170.936, p<.001, \eta_{p}^{2}=.592 ; c: F=246.715, p<.001, \eta_{p}^{2}$ $=.676$, and the delayed stage, hits: $F=56.189, p<.001, \eta_{p}{ }^{2}=.323 ; c: F=129.236, p<$ $.001, \eta_{p}^{2}=.523$. Additionally, the stimulus type by stress stage interaction was statistically significant for overall accuracy and sensitivity. Follow-up tests indicated higher overall accuracy for words as opposed to faces during the acute stress stage, $F=10.350, p=.002$, $\eta_{p}{ }^{2}=.081$, but not the delayed stage, $F=0.572, p=.451, \eta_{p}{ }^{2}=.005$. Similarly, $d^{\prime}$ 'scores for words as opposed to faces were higher during the acute stress stage, $F=9.154, p=$ $.003, \eta_{p}^{2}=.072$, but not the delayed stage, $F=0.994, p=.321, \eta_{p}{ }^{2}=.008 .{ }^{9},{ }^{10}$

Bayesian ANOVAs revealed decisive evidence for the alternative hypothesis for the main effect of stimulus on proportion of hits, proportion of false alarms, and response bias $\left(\mathrm{BF}_{10}=8.953 \mathrm{e} 36, \mathrm{BF}_{10}=4.929 \mathrm{e} 35, \mathrm{BF}_{10}=3.789 \mathrm{e} 57\right.$, respectively $)$. For the interaction between stimulus type and stress stage, there was substantial evidence for the alternative hypothesis for overall accuracy $\left(\mathrm{BF}_{10}=8.065\right)$ and sensitivity $\left(\mathrm{BF}_{10}=7.519\right)$, strong evidence for response bias $\left(\mathrm{BF}_{10}=24.390\right)$, and decisive evidence for proportion of hits $\left(\mathrm{BF}_{10}=1037.883\right)$. All other results showed more evidence towards the null hypothesis, ranging from anecdotal to substantial evidence (i.e., from $\mathrm{BF}_{01}=1.408$ to $\mathrm{BF}_{01}=9.524$ ). I report all BFs and related information in Table B.G in Appendix B.

\footnotetext{
${ }^{9}$ Analyses after removing outliers (scores $\geq 2.5$ SDs from the mean; see Table B.E in Appendix B; Ns = 113/117) returned analogous patterns of results.

${ }^{10}$ Analyses after removing guesses (responses with ratings of less than $51 \%$ confidence; see Table B.F in Appendix $\mathrm{B} ; N=120$ ) showed the same pattern of results. In addition, main effects of stress stage emerged for two of the outcome variables, false alarms and $d$ '. There was a greater proportion of false alarms $(M=.337, S E=$ $.016)$ during the acute stress stage than during the delayed stage $(M=.302, S E=.016)$. The main effect of stress stage for $d$ 'was modified by the interaction between stimulus type and stress stage.
} 
Table 3.3

Memory Performance Measures for Words and Faces as a Function of Stress Condition and Stress Stage in Experiment 3

\begin{tabular}{lcccccccc}
\hline & \multicolumn{4}{c}{ Words } & \multicolumn{3}{c}{ Faces } \\
& \multicolumn{3}{c}{ Acute } & \multicolumn{2}{c}{ Delayed } & \multicolumn{2}{c}{ Acute } & \multicolumn{2}{c}{ Delayed } \\
& Stress & No Stress & Stress & No Stress & Stress & No Stress & Stress & No Stress \\
\hline Overall & .694 & .682 & .676 & .672 & .639 & .650 & .703 & .664 \\
accuracy & $(.102)$ & $(.100)$ & $(.109)$ & $(.115)$ & $(.102)$ & $(.100)$ & $(.109)$ & $(.115)$ \\
\hline Hits (\%) & .777 & .792 & .732 & .715 & .496 & .487 & .586 & .534 \\
& $(.156)$ & $(.154)$ & $(.172)$ & $(.177)$ & $(.219)$ & $(.215)$ & $(.195)$ & $(.200)$ \\
\hline False & .396 & .398 & .372 & .370 & .212 & .179 & .180 & .206 \\
alarms (\%) & $(.180)$ & $(.177)$ & $(.164)$ & $(.161)$ & $(.156)$ & $(.154)$ & $(.164)$ & $(.169)$ \\
\hline $\boldsymbol{d}$ ' & 1.151 & 1.193 & 1.077 & 1.023 & .878 & .970 & 1.253 & 1.012 \\
& $(.656)$ & $(.653)$ & $(.656)$ & $(.653)$ & $(.719)$ & $(.714)$ & $(.719)$ & $(.714)$ \\
\hline $\boldsymbol{c}$ & .276 & .308 & .175 & .138 & -.457 & -.544 & -.390 & -.434 \\
& $(.422)$ & $(.422)$ & $(.422)$ & $(.422)$ & $(.476)$ & $(.476)$ & $(.469)$ & $(.469)$ \\
\hline
\end{tabular}

Note. $N=120 . d^{\prime}=$ sensitivity. $c=$ response bias. Acute $=$ acute stress stage. Delayed $=$ delayed stage. Numbers in parentheses represent standard deviation.w 


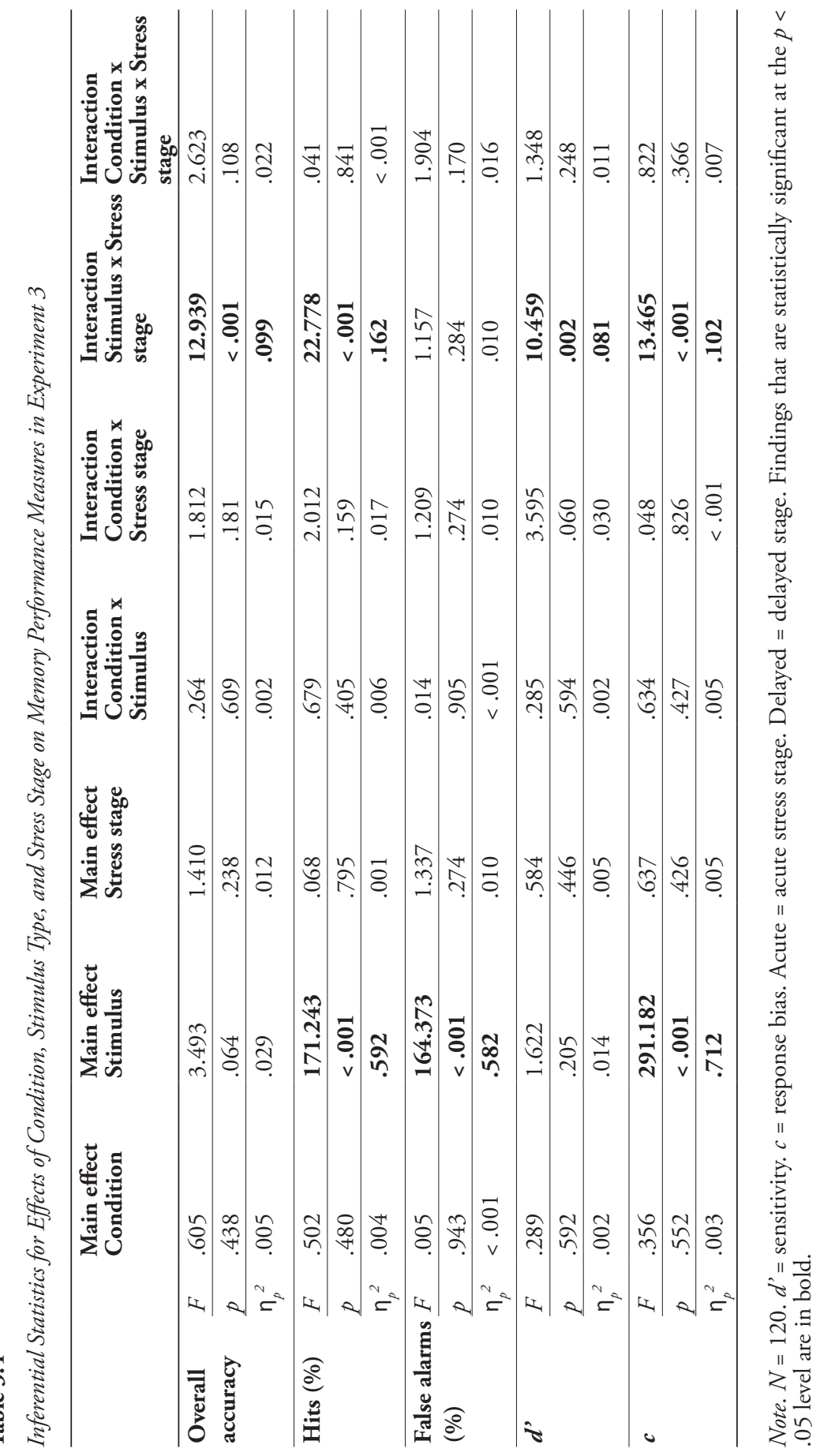




\section{Discussion}

Across two preregistered experiments, I applied contemporary methodology from the eyewitness and fundamental memory fields to study the effects of acute stress at encoding and retrieval on face recognition performance. I induced stress with a validated laboratory stressor and verified the success of the stress induction physiologically in addition to standard subjective verification. To allow for a separation of stress effects at each memory phase, I inserted a 24 to 26 -hour interval between encoding and retrieval. Contrary to my hypotheses and previous work both in the eyewitness and the fundamental memory fields, I found no effect of acute stress on face recognition memory during encoding or retrieval and no effects of encoding stress on word recognition memory. The interpretation of the findings as support of the null hypothesis were largely supported by substantial or strong evidence relying on Bayesian analyses (Jarosz \& Wiley, 2014; Jeffreys, 1961; Raftery, 1995).

My findings contrast with previous fundamental work on stress and memory showing memory enhancement for faces encoded during a stressor (Wiemers et al., 2013) and memory impairment for faces retrieved after exposure to a stressor (Li et al., 2013). At the same time, the current findings also contrast with previous eyewitness research suggesting negative effects of acute encoding stress on face recognition performance (Davis et al., 2019; Deffenbacher et al., 2004; Pezdek et al., 2020) although differences in methodology may explain these conflicting results. Indeed, the most relevant eyewitness experiment, which examined acute stress effects on identification performance using similarly robust methodology and a lineup identification task, also found no effects of acute encoding stress on memory performance (Sauerland et al., 2016). These results coupled with the current null findings call into question the robustness and generalizability of past findings regarding the effects of acute stress on face recognition memory.

The absence of effects of acute stress on word recognition is even more surprising, given the comprehensive literature supporting this idea (e.g., Abercrombie et al., 2003; Domes et al., 2002; Schwabe et al., 2008b; Zoladz et al., 2011). However, many such experiments showed enhancement effects only under certain conditions. For example, some experiments showed enhancements only for positively valenced (but not neutral or negative words; Zoladz et al., 2011) or neutral words (but not positive or negative words; Schwabe et al., 2008b). Other experiments showed effects for participants classified as high cortisol responders (e.g., Domes et al., 2002), but not for the stress group as a whole. 
However, notably, results from Experiment 3 do mirror some other fundamental work demonstrating a lack of effect of cortisone administration (de Quervain et al., 2000) or acute encoding stress (Domes et al., 2004) on word recognition performance, suggesting that such findings are not entirely atypical.

I also tested the effect of encoding stress both during and after the stress induction to examine potentially different effects of acute stress on stress stage (e.g., Quaedflieg \& Schwabe, 2018; Shields et al., 2017). Effects during the stress induction reflect the reality of an eyewitness experience during a crime, whereas effects after the stress induction reflect the time window when cortisol exerts the strongest effect on memory. One previous study found recognition enhancements for items experienced during a stressor and 41-65 minutes post-stressor (Vogel \& Schwabe, 2016). Although the stress induction was successful, I did not see any memory benefit for stressed participants in either stress stage. Timing differences might partially explain this discrepancy: the delayed stage in my study occurred around 34-39 minutes post-stressor onset, slightly earlier than the 41-65 minute period specified in this earlier experiment. I designed this study to align with the anticipated cortisol peak (Joëls \& Baram, 2009; Joëls et al., 2011; Quaedflieg \& Schwabe, 2018). Still, other research also illustrates conflicting results at various stress stages, such as showing that encoding during a stressor impaired recognition memory (Schwabe \& Wolf, 2014) or demonstrating that encoding immediately post-stressor, but not 30-minutes post-stressor, enhanced recognition of positive words (Zoladz et al., 2011). Placing my null results alongside these mixed findings highlights the need for further investigations of the specific timeline regarding effects of acute encoding stress on recognition memory.

Perhaps the most promising explanation for the lack of acute stress effects at both encoding and retrieval on recognition performance is to consider the type of memory test used. A meta-analysis examining cortisol administration effects on encoding and retrieval showed that effect sizes for recognition memory were smaller than effect sizes for free or cued recall memory (Het et al., 2005). Likewise, for retrieval stress, the effect of stress or cortisol seems to differ as a function of memory test type, with stronger negative effects for recall than recognition performance (de Quervain et al., 2000, 2003; Gagnon \& Wagner, 2016; Wolf, 2017). The underlying mechanism could be that recognition memory relies on familiarity, whereas free recall requires recollection. Recall is associated with greater hippocampal dependency than recognition (Gagnon \& Wagner, 2016). As a result, acute stress, which directly affects the hippocampus, may more strongly impair free recall, while sparing familiarity-based judgments. Thus, the type of memory test 
may influence encoding and retrieval stress effects on memory performance, perhaps explaining the null results in the current experiments. Directly comparing recognition and recall in future experiments could reveal valuable insights about the extent of effects of acute stress on different kinds of memory performance.

The current experiments also have some limitations. In Experiment 3, I found statistically significant cortisol increases in stressed participants. Examining salivary cortisol effects in Experiment 2 may also have provided a fuller picture of cortisol-related stress effects. For example, some research suggests negative effects of acute retrieval stress on memory emerge only in high cortisol responders (e.g., Buchanan et al., 2006; Buchanan \& Tranel, 2008; Schönfeld et al., 2014). Therefore, examining cortisol responder groups may have helped clarify the null effects of retrieval stress on recognition in Experiment 2. Further, in laboratory experiments-where stress inductions are constrained by ethical considerations - results reflect mild to medium levels of acute stress, rather than severe levels of stress that may be present in certain extreme criminal contexts. The face recognition tasks in Experiments 2 and 3 also differed from one another in task difficulty. Unfamiliar face recognition is already a difficult task (e.g., Hancock et al., 2000), and contrasts with other types of recognition tasks, in that participants see different images of the same faces at encoding and retrieval (as recommended, e.g., Burton, 2013), rather than seeing the exact same stimulus in both phases (e.g., as with words or images). Moreover, whereas participants saw each face twice during encoding in Experiment 2, they only encoded each face once in Experiment 3. Consequently, participants showed higher hit rates for faces in Experiment 2 than in Experiment 3. Although overall accuracy and sensitivity rates in Experiment 3 do not suggest floor effects for face recognition, the hit rates for faces in Experiment 3 were around chance level. Thus, although these hit rates are comparable to similar work with much shorter retention intervals (Davis et al., 2019; Pezdek et al., 2020), it is possible that floor effects for face recognition may not have allowed us to fully differentiate between individuals performing at such low levels for hit rate. Even so, results from Experiment 2, where hit rates did not show floor effects, similarly suggested an absence of acute stress on face recognition performance.

Other factors that could be further assessed in future research include sex/sex hormone status and stimuli valance, as past research suggest differences in stress responses depending on sex hormone status (e.g., Cahill, 2012; Hidalgo et al., 2019; Kudielka et al., 2009; Nielsen et al., 2013). Although my sample does not have enough statistical power to directly examine gender differences in a meaningful way, other samples may show reliable 
differences in cortisol responses or measures of memory performance depending on sex hormone status (cf. Shields et al., 2020). Additionally, future research could examine possible interactions between stimulus and participant gender (e.g., Herlitz \& Lovén, 2013). Furthermore, a more thorough examination of stimulus valence could advance this area of experimentation. Although meta-analyses found no evidence that stimulus valence moderates the effects of acute encoding stress on memory performance (Shields et al., 2017, but see Schwabe et al., 2008b; Zoladz et al., 2011), larger stress effects have emerged when examining retrieval stress effects on memory performance (e.g., Kuhlmann et al., 2005; Schönfeld et al., 2014). Examining the full range of valence options (e.g., neutral, positive, negative) and directly comparing faces and words would allow for a firmer conclusion. Finally, it is possible that smaller effects of acute stress on face recognition performance are present, but could not be detected in my analyses, which had power to detect medium-large (Experiment 2) or medium (Experiment 3) effect sizes.

A final limitation concerns generalizability to other populations. The current sample consisted exclusively of white participants and white target and filler faces, to limit any effects of the own-race bias (e.g., Meissner \& Brigham, 2001). Exploring these research questions in more diverse samples, as well as examining acute stress effects on face recognition in cross-race contexts, is an important future step that has not yet been addressed by empirical research. Additionally, stress may affect children (Deffenbacher et al., 2004) or older adults (Hidalgo et al., 2019; Smith et al., 2019) differently to how it affects younger adults, the population examined in the current sample. Thus, the current findings may not apply to other age groups.

Crucially, future work on acute stress and face recognition memory should examine the encoding and retrieval memory phases, both fundamentally and in more applied settings. Understanding acute stress effects during both memory phases is valuable for applied forensic settings. To do so, ensuring that suitable manipulation checks and retention intervals are in place is essential. In addition, further exploring the intricacies of the acute stress timeline (e.g., stress stage) will be important for refining our knowledge of the specific situations when acute stress may enhance, impair, or not affect face recognition performance. Finally, careful use of terminology is critical, particularly when researchers use complex scenarios where several factors are likely at play, including stress, attention, cognitive load, and other factors (e.g., Wulff \& Thomas, 2021). Disentangling these potential cumulative effects will help provide a much clearer picture on this applied topic. 


\section{Conclusion}

To conclude, these findings add to a growing body of research demonstrating an absence of stress effects on recognition performance. That is, previous eyewitness and fundamental experiments alike have obtained null effects of acute stress at encoding for face identification tasks (Sauerland et al., 2016), pictures (e.g., Goldfarb et al., 2019), and words (e.g., Domes et al., 2004). Additionally, the current results regarding retrieval stress support other findings that retrieval stress did not affect face recognition performance (Li et al., 2014). Presently, researchers are beginning to use more robust methodology to investigate stress and cognition (see Shields, 2020), and aligning with best practice methods for hypothesis testing, such as conducting informed power analyses to gather sufficient sample sizes and following preregistered analysis plans. These scientific improvements will make for a stronger evidence base in this complex literature, as reduced power can lead to overestimations in statistically significant differences. A corpus of well-powered, reliable experiments will allow for a fuller understanding of the stress-memory relationship in general and specifically when examining memory for faces. 


\title{
CHAPTER 4
}

\author{
Mitigating the Negative Effects of \\ Retrieval Stress on Memory: \\ Examining the Efficacy of an \\ Arousal Reappraisal Intervention
}

This chapter is published as the following:

Marr, C., Sauerland, M., Otgaar, H., Quaedflieg, C.W.E.M., \& Hope, L. (2021). Mitigating the negative effects of retrieval stress on memory: An arousal reappraisal intervention. Memory, 29(3), 330-344. https://doi.org/10.1080/09658211.2021.1893750 


\begin{abstract}
In a preregistered experiment, I examined the efficacy of arousal reappraisal as an intervention for reducing the negative effects of stress at retrieval on memory. Participants $(N=177)$ were semi-randomly assigned to one of three conditions: a Stress-intervention condition, a Stress-placebo condition, and a No-stress-placebo control condition. Participants viewed four images of complex, mildly negatively valenced scenes. One day later, they received an arousal reappraisal intervention or placebo before exposure to a laboratory stressor (or a control version for the No-stress condition). Participants were then tested on their memory of the images using a free recall instruction and multiplechoice recognition questions. As expected, negative affect and blood pressure increased for the stress conditions but not the control condition. Contrary to the hypotheses, memory performance did not statistically significant differ between the Stress-placebo condition and the No-stress-placebo control condition, indicating a lack of negative effects of acute retrieval stress on memory. Furthermore, there were also no statistically significant differences between the Stress-intervention condition and Stress-placebo condition in terms of memory performance, suggesting that the intervention did not assist with enhancing memory. I integrate interpretations of the findings from this study with a discussion of avenues for future research in this area.
\end{abstract}




\section{Introduction}

When a person experiences acute stress, their autonomic system is activated, leading to the release of adrenaline and symptoms such as increased heart rate, blood pressure, and sweating. In addition to this autonomic arousal, stress also activates the hypothalamicpituitary-adrenal (HPA) axis. This activation leads to the release of cortisol, which consists of both genomic and non-genomic effects (Joëls et al., 2011; Quaedflieg \& Schwabe, 2018). The non-genomic effects influence the basolateral amygdala about 15-20 minutes after an acute stressor is experienced and continue for around one hour. The genomic effects are more delayed, beginning around 60-90 minutes post-stressor and lasting hours or even days. These cortisol effects typically impair memory retrieval (de Quervain et al., 1998; Shields et al., 2017; Wolf, 2017), and these negative effects of stress on retrieval are most often found during the cortisol peak (around 20 minutes post-stressor; e.g., Schwabe $\&$ Wolf, 2014). Research has found these impairments for both recall (Kuhlmann et al., 2005b; Schönfeld et al., 2014; Smeets et al., 2008) and recognition tasks (Li et al., 2013; Schwabe \& Wolf, 2014). Notably, though, as reported in Chapter 3, I did not find any effects of acute retrieval stress on memory for faces retrieved either during or after the stressor. This may be because such effects tend to be stronger for recall than recognition (see Gagnon \& Wagner, 2016). Previous research has investigated retrieval memory for different kind of stimuli including words (Kuhlmann et al., 2005b; Schönfeld et al., 2014; Schwabe \& Wolf, 2014; Smeets et al., 2008), pictures (Schönfeld et al., 2014), and faces (Li et al., 2013). Generally, effects of retrieval stress on memory performance are larger for emotional memory compared to neutral memory (e.g., Kuhlmann et al., 2005b; Schönfeld et al., 2014).

A negative effect of acute stress before retrieval could be problematic during police interviews with witnesses. Drawing on past research concerning how acute stress negatively impacts memory retrieval (e.g., Shields et al., 2017), it is likely that witnesses who experience heightened pressure during an interview may show reduced quantity and accuracy of information. This could happen when an interview is conducted in a harsh, aggressive and suggestive manner. However, even if police are sensitive and do not explicitly set out to impose stress on witnesses during interviews, witnesses may still find the situation stressful. For example, it is possible that witnesses who are uncertain about how the interview process will transpire (Sydeman et al., 1997), believe they could be implicated in the crime, are fearful of the perpetrators they are reporting about, or suffer from social or evaluation anxiety (particularly when dealing with authority figures; 
e.g., Leitenberg, 1990) may experience increased stress levels during a police interview. Although no research has yet examined this issue in the context of police interview settings, research examining other interview settings suggests increased levels of stress for those in the interviewee position (e.g., McCarthy \& Goffin, 2004; Posthuma et al., 2002).

Recently, researchers have detected ways to reduce the negative effects of stress on memory retrieval. For example, Smith and colleagues (2016) found that participants who engaged in retrieval practice (i.e., taking practice tests) were immune to the negative effects of stress, while those who simply restudied the materials showed the typical memory impairments. Because stress levels cannot always be avoided or reduced, one tactic for managing stress is to reframe the way individuals view arousal. Research on cognitive reappraisal suggests that altering the way one interprets situations can change subsequent emotional or even physiological reactions (e.g., Lazarus, 1991). More recently, researchers have started to apply such cognitive reappraisal strategies to stressful situations. One reappraisal strategy, arousal reappraisal, has been developed from the theoretical biopsychosocial model of challenge and threat (Blascovich \& Mendes, 2010; Blascovich \& Tomaka, 1996). This model suggests that in motivated performance situations, arousal can lead individuals to either a challenge or a threat state, which exist on two opposite ends of a spectrum. In a threat state, individuals believe they do not have appropriate resources to meet the situational demands of the stressful situation. This state leads to avoidance of the situation, increased negative affect, autonomic arousal, and HPA axis activation, and impaired cognitive performance (e.g., Jamieson et al., 2018; Seery, 2011). In a challenge state, individuals believe they are able to cope with the situational demands, leading them to approach the situation. Individuals in this state report more excitement and increased self-esteem, physiologically display heightened autonomic arousal but moderate HPA activation, and show enhanced cognitive performance (e.g., Jamieson et al., 2018; Mendes \& Park, 2014). Therefore, arousal reappraisal does not decrease stress reactions, encourage relaxation, or reduce sympathetic arousal, but rather focuses on shifting the experience of stress from a threat state to a challenge state (Jamieson et al., 2018). Arousal reappraisal, consisting of advice given to individuals before they encounter stress, aims to guide individuals towards a challenge state by helping them view their feelings and bodily response to stress (e.g., sweaty palms, increased heart rate, etc.) as adaptive and useful rather than harmful.

Past research shows that arousal reappraisal helps participants perform better during 
objective laboratory stress tests compared with counterparts who do not receive arousal reappraisal instructions (e.g., giving better quality speeches during the Trier Social Stress Test; Beltzer et al., 2014). Researchers have also found similar beneficial effects in more applied stressful settings, such as on test-taking performance (e.g., performing better on standardized math examinations; Jamieson et al., 2010). These findings coupled with research showing that arousal reappraisal elicits more moderate cortisol reactions (e.g., Blascovich, 2008; Mendes \& Park, 2014) indicate that arousal reappraisal could potentially reduce the negative effects of stress before retrieval on memory. However, no research has directly investigated this possibility. Additionally, research suggests that arousal reappraisal may have other positive benefits, including reduction of negative affect and anxiety, and increasing coping ability (Jamieson et al., 2018). Though not related to memory performance, improving affect and coping ability—for example for witnesses who have just experienced a crime and a subsequent police interview-would be an added benefit of such an intervention.

The current experiment applied an arousal reappraisal intervention before a stressful situation to test the hypothesis that such an intervention can have a positive effect on recall and recognition performance. To test this research question, I included three conditions: (1) a Stress-intervention condition, in which participants received the arousal reappraisal intervention and were exposed to a stressor prior to the memory test, (2) a Stress-placebo condition, in which participants received the placebo and were exposed to a stressor prior to the memory test, and (3) a No-stress-placebo control condition, in which participants received the placebo and were not stressed prior to the memory test.

\section{Hypotheses}

1) Participants in the No-stress-placebo control condition would report more details in total, more correct details, but fewer incorrect details, and hence display better recall and recognition accuracy (proportion of correct details), and display better sensitivity (greater $d$ 'scores) than participants in the Stress-placebo condition.

2) Participants in the Stress-intervention condition would report more details in total, more correct details, but fewer incorrect details, and hence display better recall and recognition accuracy, and display better sensitivity than participants in the Stress-placebo condition. Thus, I predicted that the negative effect of retrieval stress on memory (i.e., reflected in the reporting of fewer details and less accurate information) would be reduced for those who received the intervention. 


\section{Method}

This study, including its method and analyses, was accepted in principle as a registered report. The accepted Stage 1 proposal, as well as all study materials and data, can be found at https://osf.io/8a9sq/?view_only=ce42932ad0cb415f9934b64b1d7928bf.

\section{Participants}

Based on a priori power analyses conducted using $\mathrm{G}^{*}$ Power (Faul et al., 2007), a oneway fixed-effects ANOVA with $90 \%$ power, $\nabla=.05$, and a medium effect size of $f=$ .27 (based on past relevant work including Domes et al., 2004; Hidalgo et al., 2015; Kuhlmann et al., 2005b; Schwabe \& Wolf, 2009; Schwabe \& Wolf, 2014, Experiment 2; Smeets, 2011; Tollenaar et al., 2009), the target sample size was $N=177$. Participants between the ages of 18 and 35 were recruited from the university and local community using posters, handouts/flyers, social media, and lecture visits. Consistent with relevant previous research (e.g., Quaedflieg et al., 2013; Shields, 2020; Strahler et al., 2017), I screened for and excluded participants who habitually smoked ( $>15$ cigarettes per day), drank alcohol (> 15 drinks per week), or used drugs (more than once per week). Participants were also excluded for a variety of other health reasons (i.e., BMI $<17$ and $>30$, use of medications containing cortisone, recent vaccinations, psychological treatments, cardiovascular problems, or endocrine disorders). Because sex hormones can affect cortisol reactivity (e.g., Kirschbaum et al., 1999; Strahler et al., 2017), females using hormonal contraceptives, females not using hormonal contraceptives, and males were balanced across experimental conditions. Participants' native languages were Dutch, German, or English, and they completed the memory test part of the study in their native language.

In total, 184 participants were tested. Seven participants were not included in the final sample: one because of experimental error, three because they cancelled Session 2, and three because they withdrew from participation during Session 2 (all females), noting that the task was too painful. Data collection continued until the final sample consisted of 177 participants, as planned. Of note, data were not collected during the COVID-19 lockdown in the Netherlands (March - June 2020), but some data were collected once the COVID-19 pandemic was temporarily improving (July - October 2020). During this time, participants continued to be semi-randomly distributed equally among all three groups, limiting potential COVID-19 related differences between conditions. The final sample included men $(n=32)$ and women $(n=145)$ between the ages of 18 and 34 years 
$(M=22.03, S D=3.06)$. About half the women used hormonal contraceptives $(51.72 \%)$ and the other half did not $(48.28 \%)$. The majority were university students $(87.00 \%)$, most of whom were completing their undergraduate degrees $(69.50 \%)$, with the rest completing a different degree (30.50\%).

Testing occurred between 12:00 pm and 6:00 pm to account for diurnal cortisol levels (Shields, 2020). Participants were asked to follow several rules before Session 2 of the experiment to help control for variability in cortisol levels. These instructions included not drinking alcohol the night before, getting a full night of sleep, and refraining from eating, drinking anything besides still water, exercising, smoking, or brushing teeth for at least two hours prior to the session. Participants received either course credit or 15 euros in gift vouchers as reimbursement. The three participants with the highest memory test scores (combination of greatest quantity of recall details and greatest recall and recognition accuracy) received an additional 15 euros in vouchers upon completion of data collection. This study was approved by the ethical committee of the Faculty of Psychology and Neuroscience at Maastricht University.

\section{Design}

I used a one-factorial between-subjects design with three conditions (Stress-intervention, Stress-placebo, and No-stress-placebo control; $n=59$ in each condition). The dependent variables were recall quantity (total number of details, total number of correct details, and total number of incorrect details), recall accuracy (number of correct details divided by total number of details reported), recognition accuracy (proportion of correct answers), recognition sensitivity $(d)$, and response bias $(c)$. Participants answered recall questions for two images and recognition questions for two images. The order of recall and recognition questions was counterbalanced.

\section{Materials}

\section{Stimuli and Retrieval Test}

Four color images depicting complex drawings with object- and person-related details were used (Kitchen, Living Room, Traffic, and Bus), displaying mildly negative scenes (e.g., a person falling over, a flat tire, etc.). These images can be found in Appendix C. Based on previous work using these materials (see Sauer \& Hope, 2016), each image was shown for 60 seconds with a 10-second interval. The recall test for two of these images 
(i.e., Kitchen and Traffic) consisted of an open-ended invitation to report everything they could remember about the images (including people, events, and actions) in as much detail and as accurately as possible. Participants were discouraged from guessing. The recognition test for the other two images (i.e., Living Room and Bus) consisted of 25 questions per image. Each question was presented with five multiple-choice options (e.g., "What color were the painter's clothes?" a) Blue, b) Red, c) Orange, d) Green, e) None of the above). The fifth choice was always "None of the above" and this option was correct $20-25 \%$ of the time. Recognition scores were coded as the proportion of correct answers (i.e., recognition accuracy). Proportions of hits and false alarms were also calculated. Recognition sensitivity (d) was calculated as the difference between the z-scores of proportion of hits and proportion of false alarms, where the larger the $d$ ', the better one's performance. Response bias $(c)$ was calculated as the sum of the z-scores of hits and false alarms divided by two, where 0 represents no bias, values $<0$ represent a liberal bias, and values $>0$ represent a conservative bias. Each participant answered recall questions for two of the images and recognition questions for the other two images, that is, they gave two free recall accounts and answered 50 recognition questions in total.

\section{Inter-coder Reliability}

To ensure appropriate levels of inter-coder reliability for free recall, a systematic coding protocol was used, with two coders trained for each language (six total). The coding protocol included a list of possible items that may be reported (including person details, objects, actions, etc.) and coders coded against this list, updating and adding details if necessary. Two coders coded $34 \%$ of the free recall responses. Intraclass coefficient correlations (ICCs) were calculated based on a single rating, absolute-agreement, twoway random effects model. ICCs ranged between .680 and .938, indicating moderate to excellent reliability (Koo $\& \mathrm{Li}, 2016$ ). Table C.A in Appendix C shows the full ICCs analyses.

\section{Maastricht Acute Stress Test}

Participants in the stress conditions were exposed to the Maastricht Acute Stress Test (MAST), a validated laboratory stressor that combines blocks of hand immersion into ice-cold water (four degrees Celsius) with socially-evaluated mental arithmetic in front of a critical experimenter who gives negative feedback throughout the task (see Smeets et al., 2012). Additionally, as part of the MAST, participants were told they were being video recorded for later facial expression analysis. Participants in the No-stress-placebo control 
condition were exposed to the control version of this task, involving hand immersion into room-temperature water (35 degrees Celsius) and basic counting, with no mention of video recording.

\section{Positive and Negative Affect Schedule (PANAS)}

The Positive and Negative Affect Schedule (PANAS; Watson et al., 1988) consists of two affect scales that each contain 10 items to measure positive and negative affect. Participants indicate to what extent they feel a certain mood (e.g., interested, excited, nervous, distressed, etc.) at the present moment on a 5-point Likert scale (from very slightly or not at all to extremely). The PANAS was used to measure positive and negative affect at five timepoints: during Session 1, at the start of Session 2, after the intervention (or placebo), after the (control) MAST, and after the memory test.

\section{Blood Pressure}

To examine autonomic nervous system activation, systolic and diastolic blood pressure were collected using an Omron Blood Pressure Monitor 705IT (for validation see Coleman et al., 2006). Blood pressure was measured ten times throughout the procedure: during Session 1, baseline during Session 2, before the intervention (or placebo), during the intervention (or placebo), right after the intervention (or placebo), before the (control) MAST, during the (control) MAST, right after the (control) MAST, before the memory test, and during the memory test.

\section{Arousal Reappraisal and Placebo Interventions}

Arousal reappraisal materials were adapted from past work (Jamieson et al., 2016, 2018) and are presented in Appendix C. These materials included summaries of three scientific articles pertaining to what happens when we experience stress. In the arousal reappraisal intervention, these article summaries emphasized the adaptive benefits of stress and suggested that perceiving such arousal as adaptive would enhance performance. For example, the intervention stated Bodily reactions to a challenge are adaptive and good and An increased heart rate helps deliver fuel to your brain to help you think. In the placebo version, these articles suggested that ignoring the symptoms of arousal would help performance. For example, the placebo stated Not thinking about the possible negative outcomes can help you remain calm during difficult testing situations or If you find yourself feeling anxious during the task today, remember that you will not feel as bad if you ignore these 
negative thoughts of anxiety. After each of the article summaries, participants responded to a written prompt (i.e., In your own words please briefly describe how this information can help you perform well on the upcoming task) to ensure their engagement with the intervention or placebo materials.

\section{Stress Appraisal}

A validated measure to evaluate stress appraisals (Mendes et al., 2007) was used three times throughout the procedure: during Session 1, during Session 2 right before the MAST (but post-intervention and MAST instructions), and after the memory test. The task was described to participants as the (control) MAST and the subsequent memory test. Items in the final time point were written in past tense. The questionnaire consists of 11 questions with responses ranging on a scale from 1 (strongly disagree) to 7 (strongly agree) and is composed of two subscales: task demands and coping resources $\left(\alpha_{\text {demand }}=.86\right.$, $\alpha_{\text {resource }}=.78$ ). The six task demand questions focus on perceptions of danger, uncertainty, and required effort (i.e., "This task is demanding"; "This task is stressful"; "This task is distressing"; "This task is threatening"; "I am uncertain how I will perform"; "This task requires a lot of effort"). The five coping resources questions focus on perceptions of safety and familiarity of the situation, skills, knowledge, and abilities (i.e., "I have the abilities to perform well"; "I have the expectations to perform well"; "Performing well is important to me'; "This task is a positive challenge"; "I am the type of person who does well on these tasks"). Responses were averaged separately for the task demand questions and the coping resources questions, and a threat index was created by calculating the demands/ resources ratio.

\section{Mindfulness Assessment}

Because dispositional mindfulness has been associated with increased coping ability and decreased cortisol reactivity (e.g., Bergomi et al., 2013; Daubenmier et al., 2014), I used the Kentucky Inventory of Mindfulness Skills (KIMS; Baer et al., 2004) to assess mindfulness techniques. This measure is comprised of 39 items that are rated on a scale from 1 (Never or very rarely true) to 5 (Very often or always true). It assesses four mindfulness skills: Observing $(\alpha=.82)$, Describing $(\alpha=.92)$, Acting with awareness ( $\alpha$ $=.79)$, and Accepting without judgment $(\alpha=.89)$. Participants completed this measure during Session 1 before the encoding began. 


\section{Procedure}

During Session 1, after filling out the informed consent form, participants completed the stress appraisal questionnaire and the KIMS. After a blood pressure measure, they completed the PANAS. Next, participants viewed the images. To ensure engagement and motivation, they were first informed that the top three scorers on the later test would each receive an additional 15-euro voucher upon completion of the study. Session 1 ended with another PANAS. The next day, participants returned to the lab (24-26 hours later) for Session 2 and completed a demographic questionnaire as well as other baseline data measures, including subjective stress. After 10 minutes had passed since they started, participants gave a baseline blood pressure and completed the PANAS. Participants then received the placebo or actual intervention manipulations. Participants were asked to rephrase each summary in writing in their own words to ensure engagement with the task. These tasks took around 10 minutes to complete. Next, participants completed the PANAS and engaged in the instruction section of the MAST before completing the stress appraisal questionnaire. They next engaged in the (control) MAST. Similar intervals $(\sim 5$ minutes) between the intervention and relevant tasks have been used in past research (e.g., Jamieson et al., 2016). Next, participants again completed the subjective stress questionnaires. After 10 minutes had passed since the end of the MAST (to assess memory retrieval during the cortisol peak; Smeets et al., 2012), participants began the memory test. First, the experimenter explicitly reminded participants to continue to utilize the information they learned in the summaries of the scientific articles. Partway through data collection, experimenters also began to remind participants to use their native language, because some participants filled out the memory test in the wrong language. Participants were then tested on their memory for the images using free recall (for two images) and multiple-choice recognition questions (for two images). Finally, participants once again completed subjective questionnaires focusing on subjective stress ratings and the stress appraisal questionnaire. Blood pressure was measured ten times throughout the session. Figure 4.1 shows an overview of the timeline. 
Figure 4.1

Study Timeline and Procedure

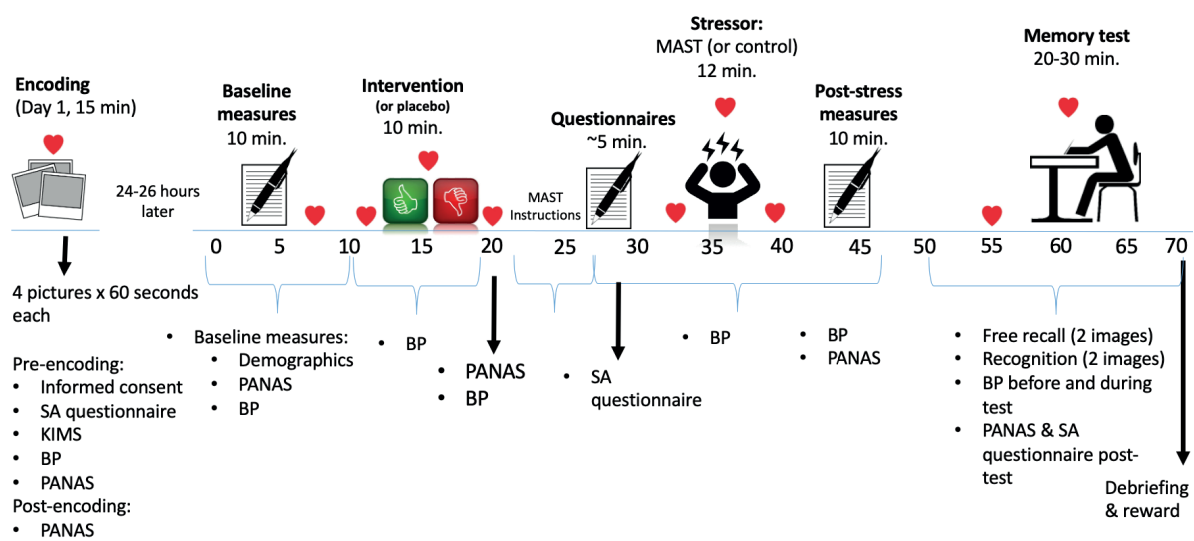

Note. $\mathrm{SA}=$ stress appraisal. KIMS $=$ Kentucky Inventory of Mindfulness Skills. PANAS $=$ Positive and Negative Affect Schedule. and BP = blood pressure. MAST = Maastricht Acute Stress Test.

\section{Results}

\section{Manipulation Checks}

To assess the successfulness of the stress induction, I compared subjective stress (PANASPositive Affect and Negative Affect scores; six within-levels) and blood pressure (systolic and diastolic; ten within-levels) between the Stress-intervention, Stress-placebo, Nostress-placebo control conditions using a mixed ANOVA. I also computed a mixed ANOVA to examine stress appraisal responses (scored separately for task demands and coping resources and combined into a threat index by dividing the two; three withinlevels).

\section{Affect}

Higher scores on the positive affect scale of the PANAS reflect higher self-reported positive affect, and higher scores on the negative affect scale of the PANAS reflect higher selfreported negative affect. Stress differentially affected negative affect scores depending on timing, $F(6.886,174)=14.561, p<.001, \eta_{p}^{2}=.143$. Follow-up tests revealed differences between conditions pre-stressor: during Session 1 pre-encoding, $F(2,174)=4.555$, $p$ $=.012, \eta_{p}^{2}=.050$, Session 1 post-encoding, $F(2,174)=3.159, p=.045, \eta_{p}^{2}=.035$, and Session 2 baseline, $F(2,174)=5.316, p=.006, \eta_{p}^{2}=.058$. Pairwise comparisons 
indicated that negative affect scores in the Stress-intervention condition were statistically significantly higher than in the No-stress-placebo control condition during Session 1 preencoding $(p=.011)$. Likewise, the Stress-intervention condition showed higher negative affect scores than both the No-stress-placebo control and Stress-placebo conditions at Session 2 baseline ( $p=.015$ and $p=.017$, respectively). Due to the unexpected initial difference in negative affect between groups in Session 1, an exploratory ANCOVA analysis was also conducted, examining the Session 1 pre-encoding negative affect scores as a covariate. These results showed a significant interaction between stress and timing, $F(6.183,534.835)=17.126, p<.001, \eta_{p}^{2}=.165$, though there was no longer a statistically significantly difference between conditions in the Session 1 post-encoding and Session 2 baseline negative affect scores.

In addition and as expected, I observed differences post-MAST, $F(2,174)=31.292$, $p$ $<.001, \eta_{p}^{2}=.265$. All conditions differed post-MAST (Stress-intervention vs. Stressplacebo: $p<.001$; Stress-intervention vs. No-stress-placebo control: $p<.001$; Stressplacebo vs. No-stress-placebo control: $p=.001)$, with the Stress-intervention condition showing the highest negative affect scores $(M=20.627, S E=.754)$, followed by the Stress-placebo condition $(M=16.102, S E=.754)$, and finally, the No-stress-placebo control condition $(M=12.203, S E=.754)$. For positive affect, there was no statistically significant difference between conditions, $F(8.424,174)=1.017, p=.422, \eta_{p}^{2}=.012$. Figure 4.2 displays changes in negative and positive affect scores over time. 
Figure 4.2

Negative and Positive Affect Scores over Time per Condition
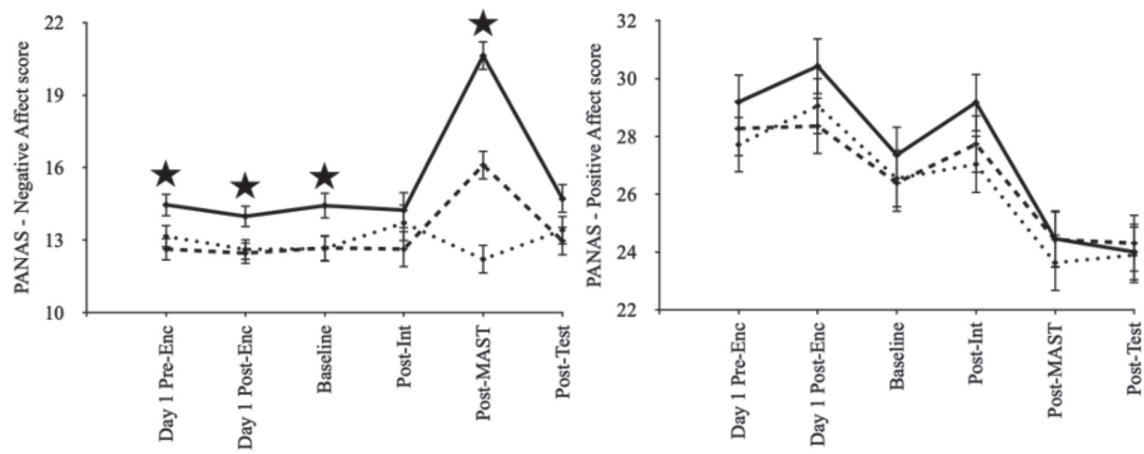

- Stress-intervention

- - Stress-placebo

- No-stress

Note. Different Y-axis labels for display purposes. PANAS = Positive and Negative Affect Schedule (Watson et al., 1988). Enc $=$ Encoding. Int $=$ Intervention/Placebo. MAST $=$ Maastricht Acute Stress Test. Test $=$ memory test. Error bars $=$ standard error. $\star=p<.05$. Possible negative and positive affect scores range between 10 and 50 .

\section{Blood Pressure}

Stress differentially affected blood pressure depending on the timing for both systolic and diastolic measures, $F(11.622,174)=13.157, p<.001, \eta_{p}^{2}=.131$ and $F(12.136$, 174) $=15.094, p<.001, \eta_{p}^{2}=.148$, respectively. Follow-up tests revealed no statistically significant differences between conditions at baseline, systolic: $F(2,174)=1.238, p=$ $.292, \eta_{p}^{2}=0.014$, diastolic: $F(2,174)=.052, p=.949, \eta_{p}^{2}<.001$. However, during the stressor (i.e., MAST), the stress conditions showed statistically significantly higher blood pressure than the non-stressed condition, systolic: $F(2,174)=25.208, p<.001, \eta_{p}^{2}=.225$ (Stress-intervention vs. Stress-placebo: $p>$.999; Stress-intervention vs. No-stress-placebo control: $p<.001$; Stress-placebo vs. No-stress-placebo control: $p<.001)$, diastolic: $F(2$, $174)=32.672, p<.001, \eta_{p}^{2}=.273$ (Stress-intervention vs. Stress-placebo: $p>.999$; Stress-intervention vs. No-stress-placebo control: $p<.001$; Stress-placebo vs. No-stressplacebo control: $p<.001$ ). Figure 4.3 shows changes in blood pressure measurements across time. 


\section{Stress Appraisal}

A threat index was created by calculating the demands/resources ratio from the Stress Appraisal subscales, with numbers $>1$ representing task demands outweighing coping resources. Stress condition differentially affected the threat index depending on the timing, $F(3.712,174)=19.624, p<.001, \eta_{p}^{2}=.184$. Follow-up tests revealed no statistically significant differences during Session $1, F(2,174)=.677, p=.510, \eta_{p}^{2}=.008$. Pre-MAST, both Stress conditions showed a higher threat index than the No-stress-placebo control condition, $F(2,174)=52.255, p<.001, \eta_{p}^{2}=.375$ (Stress-intervention vs. Stress-placebo: $p>$.999; Stress-intervention vs. No-stress-placebo control: $p<.001$; Stress-placebo vs. No-stress-placebo control: $p<.001)$. Additionally, but to a lesser extent, the Stressintervention condition showed a higher threat index than the No-stress-placebo control condition Post-test $(p<.001), F(2,174)=9.775, p<.001, \eta_{p}^{2}=.101$. Figure 4.4 shows changes in threat index over time. Table C.B in Appendix C shows exploratory analyses of stress effects on task demands and coping resources subscales separately.

\section{Figure 4.3}

Systolic and Diastolic Blood Pressure over Time per Condition
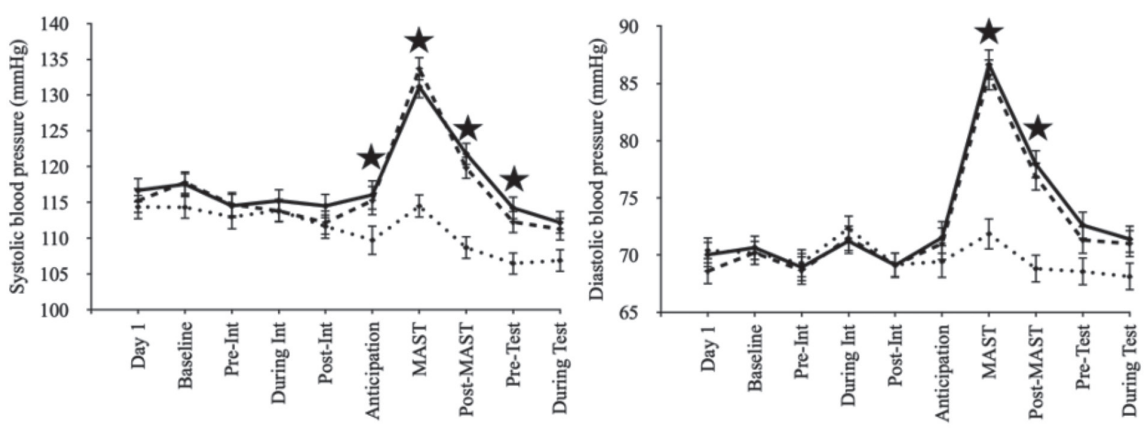

- Stress-intervention
- - - Stress-placebo

\section{- No-stress}

Note. $\mathrm{mmHg}=$ millimeters of mercury. Int = Intervention/Placebo. Anticipation = Anticipation phase of the MAST. MAST = Maastricht Acute Stress Test. Test = memory test. Error bars = standard error. $\boldsymbol{\downarrow}=p<.05$. For each significant indicator, both stress conditions showed higher blood pressures than the no-stress condition, apart from the Anticipation phase for systolic, in which the Stress-intervention condition showed a statistically significant higher systolic blood pressure than the No-stress-placebo control condition $(p=.030)$ but the Stress-placebo condition did not $(p=.073)$. 
Figure 4.4

Threat Index (Demands/Resources) over Time per Condition

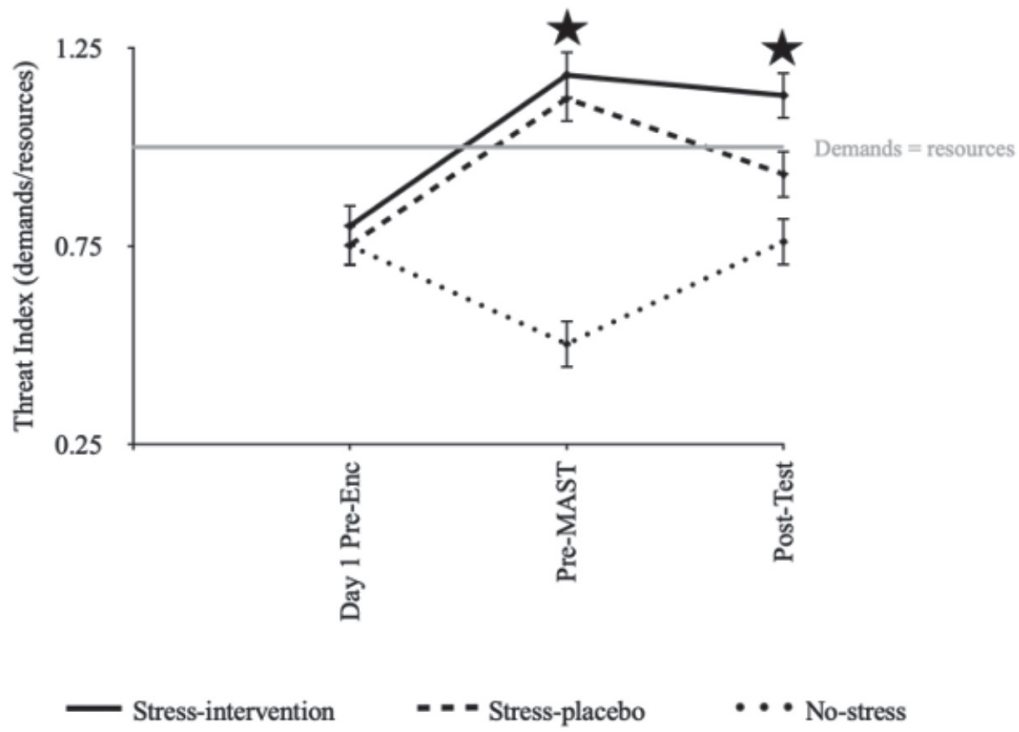

Note. An index $>1$ represents task demands outweighing coping resources. Enc $=$ encoding. MAST $=$ Maastricht Acute Stress Test. Test $=$ memory test. Error bars $=$ standard error. $\downarrow=p<.05$. At pre-MAST, both Stress conditions statistically significantly differed from the No-stress-placebo control condition $(p s<.001)$. Post-test, only the Stress-intervention and No-stress-placebo control conditions statistically significantly differed from each other $(p<.001)$.

\section{Main Analyses: Effect of Stress and Intervention on Memory Performance}

I examined the effect of stress and intervention condition on recall quantity (number of total details, correct details, and incorrect details), recall accuracy, recognition accuracy, and recognition sensitivity in separate one-way ANOVAs. ${ }^{11}$ In planned comparisons using two-tailed tests, I compared performances in the Stress-placebo vs. No-stressplacebo control conditions (i.e., Hypothesis 1) and Stress-placebo vs. Stress-intervention conditions (i.e., Hypothesis 2).

Eighteen participants completed the free recall task incorrectly, using English rather than

\footnotetext{
${ }^{11}$ In light of the unexpected baseline differences in negative affect between conditions, I also ran ANCOVAs for each main analysis with baseline negative affect as a covariate. Controlling for baseline negative affect did not alter results; these additional analyses are reported in Table C.C in Appendix C.
} 
their native Dutch or German language $(n=3)$, describing the wrong image $(n=13)$, or failing to provide a free recall response for one of the images $(n=2)$. These participants were excluded from the free recall analyses, leaving $N=159$ for these analyses and $N=$ 177 for recognition analyses.

Descriptives and inferential statistics regarding stress effects on memory measures are reported in Table 4.1. In contrast with my predictions, I found no statistically significant differences in recall quantity or recall accuracy as a function of condition. Recognition accuracy and recognition sensitivity $(d)$ also did not differ statistically significantly as a function of condition (see Figures 4.5 and 4.6). As shown in Table 4.2, planned multiple comparisons for each memory measure revealed no statistically significant differences between any condition. Thus, in line with the overall test, I did not find support for Hypothesis 1 or Hypothesis 2 in the planned multiple comparisons.

In the planned exploratory analysis of response bias $(c)$ where 0 represents no bias, values $<0$ represent a liberal bias, and values $>0$ represent a conservative bias, I found a small but statistically significant effect of stress on response bias, $F(2,176)$ $=3.838, p=.023, \eta_{p}{ }^{2}=.042$. Planned exploratory multiple comparisons using Tukey HSD (see Table 4.2) showed a statistically significant difference between the Stressintervention and the No-stress-placebo control conditions $(p=.031)$. The Stressintervention condition showed a more conservative response bias than the No-stressplacebo control condition, though responding was generally more conservative than liberal across all conditions (i.e., above 0 ; less likely to choose a definitive answer, more likely to select the "None of the above" option). 
Table 4.1

Descriptive and Inferential Statistics for Memory Performance Measures

\begin{tabular}{lccccccccc}
\hline & \multicolumn{3}{c}{ Stress-intervention } & \multicolumn{2}{c}{ Stress-placebo } & \multicolumn{2}{c}{ No-stress-placebo } & \multicolumn{3}{c}{ Stress effects } \\
\hline Free recall & $M$ & $S D$ & $M$ & $S D$ & $M$ & $S D$ & $F$ & $p$ & $\eta_{p}^{2}$ \\
\hline Quantity & 70.745 & 19.340 & 72.596 & 23.015 & 69.365 & 18.028 & .334 & .716 & .004 \\
\hline Accuracy & .877 & .072 & .863 & .085 & .880 & .079 & .657 & .520 & .008 \\
\hline Recognition & & & & & & & & & \\
\hline Accuracy & .548 & .100 & .518 & .114 & .535 & .107 & 1.172 & .312 & .013 \\
\hline Sensitivity & -.029 & .729 & -.163 & .720 & .061 & .672 & 1.499 & .226 & .017 \\
\hline Response bias & .235 & .320 & .216 & .289 & .100 & .245 & $\mathbf{3 . 8 3 8}$ & $\mathbf{. 0 2 3}$ & $\mathbf{. 0 4 2}$ \\
\hline
\end{tabular}

Note. Reported results are from one-way ANOVAs with condition (3 groups) as between-subjects factor. Free recall quantity $=$ number of total reported details. Free recall accuracy $=$ correct reported details/total reported details. Recognition accuracy $=$ correct answers/all questions. Recognition sensitivity $=d^{\prime}$. Recognition response bias $=c$. Findings that are statistically significant at the $p<$ .05 level are in bold.

Table 4.2

Planned Multiple Comparisons for Memory Performance Measures

\begin{tabular}{lccc}
\hline & $\begin{array}{c}\text { Stress-placebo vs. } \\
\text { no-stress-placebo control } \\
\text { (Hypothesis 1) }\end{array}$ & $\begin{array}{c}\text { Stress-intervention vs. } \\
\text { stress-placebo } \\
\text { (Hypothesis 2) }\end{array}$ & $\begin{array}{c}\text { Stress-intervention vs. } \\
\text { no-stress-placebo control } \\
\text { (Exploratory) }\end{array}$ \\
\hline Free recall & $p$ & $p$ & $p$ \\
\hline Quantity & .695 & .884 & .934 \\
\hline Accuracy & .529 & .652 & .976 \\
\hline Recognition & & & .771 \\
\hline Accuracy & .678 & .280 & .769 \\
\hline Sensitivity & .200 & .559 & .031 \\
\hline Response bias & .073 & .934 & \\
\hline
\end{tabular}

Note . Correction $=$ Tukey HSD. Free recall quantity $=$ number of total reported details. Free recall accuracy $=$ correct reported details/total reported details. Recognition accuracy $=$ correct answers/all questions. Recognition sensitivity $=d^{\prime}$. Recognition response bias $=c$. Findings that are statistically significant at the $p<.05$ level are in bold. 


\section{Figure 4.5}

Free Recall Performance across Stress Conditions
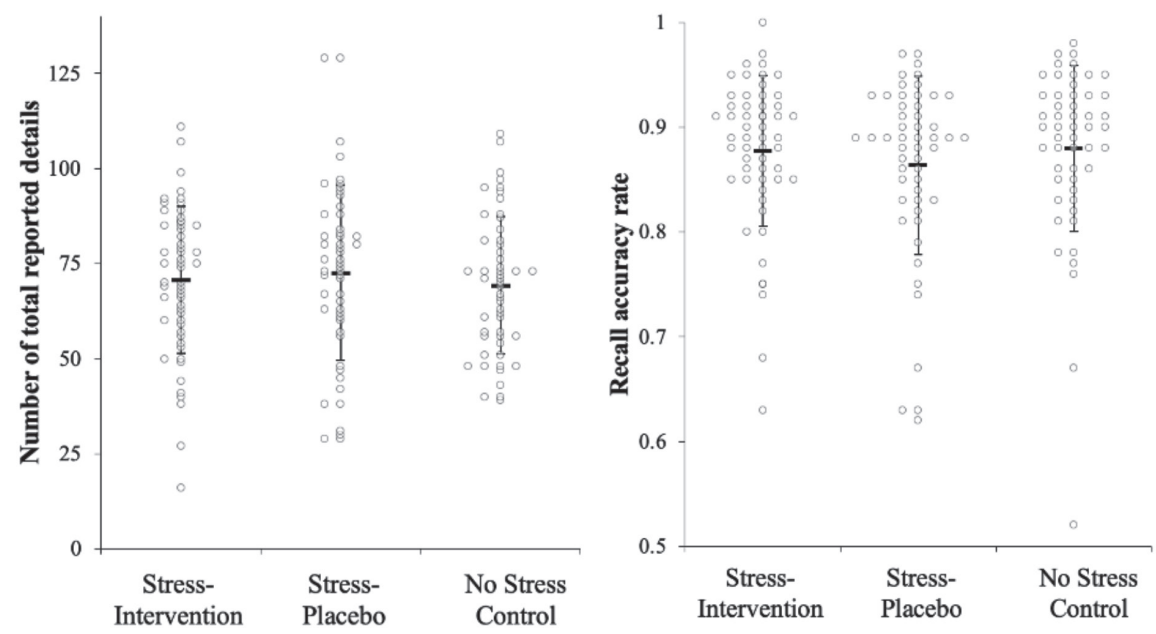

Note $=$ Mean. Errors bars $=$ standard deviation. Figures created using template from Weissgerber et al. (2015).

\section{Figure 4.6}

Recognition Performance across Stress Conditions
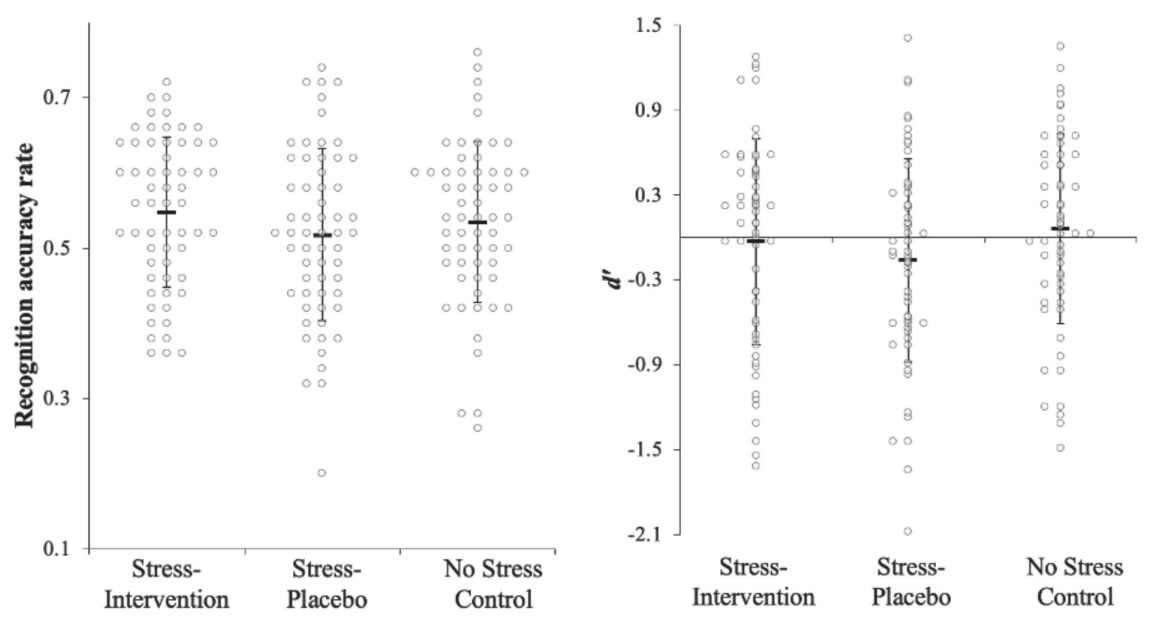

Note $=$ Mean. Errors bars $=$ standard deviation. Figures created using template from Weissgerber et al. (2015). 


\section{Planned Exploratory Analyses}

\section{Dispositional Mindfulness Moderation Analysis}

To examine whether participants' dispositional mindfulness moderated the relationship between acute stress and memory performance, I conducted four moderated regression analyses using the PROCESS v.3.4.1 for SPSS (Model 1; Hayes, 2018). I entered Stress condition into the model as the predictor (X; 1 = Stress-intervention, 2 = Stress-placebo, 3 = No-stress-placebo control), memory measures as the outcome variable (Y; i.e., recall quantity, recall accuracy, recognition accuracy, recognition sensitivity), and KIMS score (overall $M=126.508, S D=13.751$ ) as the moderator $(\mathrm{W})$.

Table C.D in Appendix C presents a summary of these moderator analyses. The overall models neither predicted a significant amount of variance in recall quantity, $F(3,155)$ $=1.112, p=.346, R^{2}=.021$, nor recall accuracy, $F(3,155)=.131, p=.942, R^{2}=.003$. Similarly, the overall models neither predicted a significant amount of variance in recognition accuracy, $F(3,173)=.455, p=.714, R^{2}=.008$, nor recognition sensitivity, $F(3,173)=.159, p=.924, R^{2}=.003$. Again, neither interaction term was significant. Thus, dispositional mindfulness did not moderate the relationship between acute stress and memory performance.

\section{Bayes Factor}

As planned, I used JASP version 0.13.1 (JASP Team, 2020) with the default Cauchy prior (.707) to conduct Bayesian independent samples t-tests between the Stress-intervention and the No-stress-placebo control condition on recall quantity, recall accuracy, recognition accuracy, and recognition sensitivity. I found substantial evidence supporting the null hypothesis across all memory measures (recall quantity: $\mathrm{BF}_{01}=4.578$, error $\%=0.018$; recall accuracy: $\mathrm{BF}_{01}=4.782$, error $\%=0.016$; recognition accuracy: $\mathrm{BF}_{01}=4.061$, error $\%=0.0004$; recognition sensitivity: $\mathrm{BF}_{01}=4.098$, error \% = 0.0005; Jarosz \& Wiley, 2014; Jeffreys, 1961; Raftery, 1995).

\section{Discussion}

In this preregistered experiment, I examined the usefulness of an arousal reappraisal intervention for mitigating negative stress effects on memory performance. I predicted that retrieval stress would impair recognition and recall memory (Hypothesis 1) and that an arousal reappraisal intervention would mitigate these negative effects of retrieval stress on memory (Hypothesis 2). Although the stressor effectively increased blood pressure 
and negative affect scores, I did not find statistically significant effects of retrieval stress on recognition or recall memory performance. Furthermore, the arousal reappraisal intervention showed no statistically significant effects on the outcome memory measures. These findings do not support Hypotheses 1 and 2.

\section{Effects of Retrieval Stress on Memory Performance}

As expected and in line with previous research (e.g., Shilton et al., 2017; Smeets et al., 2012), negative affect and blood pressure increased in both stress conditions after experiencing the stressor, but did not increase in the control condition. However, unexpectedly, self-reported negative affect scores were higher in the Stress-intervention group even during Session 1 and at baseline during Session 2. Because participants all experienced the same procedure up until reading the intervention or placebo packets, these results cannot be explained by differences in experimental condition, particularly because my exploratory analyses reveal that the unexpected Session 2 baseline differences were driven by the initial Session 1 pre-encoding differences that took place just a few minutes into Session 1 testing. Regardless of this anomaly, the results confirm the efficacy of the MAST at increasing subjective stress and physiological arousal in the stress conditions but not the control condition.

I found no support for Hypothesis 1, as there were no statistically significant differences between the Stress-placebo and the No-stress-placebo control condition for any of the memory measures. Most published past research demonstrated negative effects of retrieval stress on memory and a recent meta-analysis, examining 26 published papers, also surmised that acute stress at retrieval impaired memory performance (Hedges' $g=$ -.22; Shields et al., 2017). Still, some experiments only show these impairing effects under certain conditions. For example, past experiments have suggested stronger stress effects for recall as opposed to recognition (de Quervain et al., 2000, 2003; Gagnon \& Wagner, 2016). I examined both types of memory test, as research has shown that retrieval stress can affect both types of memory measures (e.g., Kuhlmann et al., 2005; Schönfeld et al., 2014; Schwabe \& Wolf, 2014). However, the current data show that acute retrieval stress experienced before the memory test did not statistically significantly affect performance for either test type. It is also possible that retrieval stress effects may only be apparent when examining cortisol responders, but not the stress condition as a whole (e.g., Buchanan et al., 2006; Buchanan \& Tranel, 2008; Schönfeld et al., 2014). Other research suggests impairing effects in samples of men (e.g., Kuhlmann et al., 2005b), but not women (e.g., 
Hidalgo et al., 2015; Schoofs \& Wolf, 2009). Relatedly, meta-analytic effects of retrieval stress on memory performance were only statistically significant when examining studies that excluded women using hormonal contraceptives (Shields et al., 2017).

Some research is more in line with the current findings, showing null effects of retrieval stress on memory. Specifically, stress had no effect on recognition and recall memory of men and women for word lists, autobiographical memories, and videos as stimuli (Beckner et al., 2006; Schoofs \& Wolf, 2009; Tollenaar et al., 2009; Zoladz et al., 2014). Therefore, research on retrieval stress and memory performance does show mixed findings on related research questions. Thus, although not consistent with the hypotheses, my findings add to existing evidence that effects of acute retrieval stress on memory performance may not be robust under all conditions.

\section{Effects of Arousal Reappraisal Intervention on Negative Affect and Stress Appraisals}

In addition to examining negative affect as a stress manipulation check, I investigated whether the arousal reappraisal intervention influenced negative affect and stress appraisals. Past research suggested that arousal reappraisal interventions decrease negative affect (e.g., Jamieson et al., 2013a, 2018; Seery, 2011) and increase positive affect (Jentsch $\&$ Wolf, 2020). However, the current data do not support this idea, with the Stressintervention condition displaying higher negative affect than even the Stress-placebo condition post-MAST. In fact, I also found that this post-MAST difference between the stress conditions was not driven by the initial group difference at pre-encoding in Session 1 , strengthening the idea that the intervention did not reduce negative affect. My findings do align with past work that similarly showed no change in negative or positive affect for participants engaging in an arousal reappraisal intervention in both a socially evaluated speech task and a painful cold pressor task (Denson et al., 2014). Thus, these findings in sum suggest that arousal reappraisal does not consistently alter positive and negative affect, at least across a variety of laboratory stressors, including the modified TSST, a cold pressor task, and the MAST.

The current findings also do not support the notion that the arousal reappraisal intervention increases coping resources compared to the placebo. In fact, the Stressintervention condition showed the highest threat indices. Both pre-MAST and posttest, the Stress-intervention condition differed statistically significantly from the control 
condition and showed a threat index above 1, suggesting that participants felt the task demands outweighed their coping resources. The Stress-placebo condition showed a similar difference with the control condition pre-MAST, but not post-test. Notably, the Stress-intervention condition did not statistically significantly differ from the Stressplacebo condition at any timepoint. These findings are inconsistent with past results suggesting that arousal reappraisal interventions lower the threat index by increasing self-reported coping resources (Beltzer et al, 2014; Jamieson et al., 2012, 2013b, 2016). However, they are in line with other work showing no such effects of arousal reappraisal on anticipatory stress appraisals (Jentsch \& Wolf, 2020). Thus, perhaps the effects of arousal reappraisal interventions on subjective affect and coping resources are not wholly effective across different settings.

My research differed from previous work examining arousal reappraisal on several few key variables, which may help explain the inconsistent results. The type of stressor used in this experiment may explain the reduced efficacy of the arousal reappraisal intervention (Denson et al., 2009; Liu et al., 2019). The MAST uses a combination of social-evaluative threat and pain-based stress. Specifically, results from a recent meta-analysis showed that arousal reappraisal interventions improved subjective stress outcomes for active stressors (e.g., those requiring participant engagement, such as performing a speech) but not for passive stressors (e.g., those requiring minimal engagement, such as hand submersion into cold water; Liu et al., 2019). However, other research suggests that reappraising passive, pain-based stressors is possible (Bray, 2015), with participants who engaged in arousal reappraisal displaying increased coping resources when confronted with painbased stressors (Denson et al., 2014). Still, the passive sections of the MAST may have contributed to the null results observed in the current study. Focusing on changing stress mindsets may be better suited for more passive, pain-based tasks, by teaching participants about more general beliefs about the nature of stress rather than manipulating specific appraisals of demands and resources. For example, instructing participants to take on mindsets that stress generally enhances ability can promote adaptive behaviors and less negative outcomes (Crum et al., 2013, 2017; Jamieson et al., 2018). Thus, even when resources are not sufficient to meet the demands of the situation (e.g., the pain of the MAST), improving performance using reappraisal may still be conceivable. 


\section{Effects of Arousal Reappraisal Intervention on Memory Performance}

With data suggesting a null effect of acute retrieval stress on memory, I could not examine whether arousal reappraisal can mitigate these effects. However, I was still able to test whether the Stress-intervention group showed enhanced memory performance by comparing the stress conditions as planned. There were no statistically significant differences between the Stress-intervention and Stress-placebo condition on any of the memory measures, indicating no support for Hypothesis 2. Additionally, the planned exploratory analyses revealed no evidence that dispositional mindfulness moderated the relationship between acute stress and memory performance, contrary to recent research that showed that greater habitual use of reappraisal leads to more adaptive cardiovascular responses for those engaging in an arousal reappraisal intervention (Jentsch \& Wolf, 2020). Finally, I found substantial Bayesian evidence that there was no difference between the Stress-intervention and control condition.

Other previous null findings regarding arousal reappraisal interventions may point to explanations for the current data. Although reappraising arousal can be beneficial for certain cognitive tasks, perhaps it is not useful for all types of cognitive tasks. For example, arousal reappraisal improves executive functioning (Jamieson et al., 2010, 2013a). The authors suggest that perhaps arousal reappraisal benefited quantitative but not qualitative performance in their experiment because math problems rely on more active processing using executive resources than verbal problems. The same idea may apply to various memory tasks. For example, while free recall requires active recollection, recognition relies on familiarity (Gagnon \& Wagner, 2016). As recollection requires greater executive functioning than familiarity (McCabe et al., 2010), perhaps arousal reappraisal would not benefit recognition tasks. However, I neither saw effects of stress nor the intervention on recall memory performance, where executive functioning and active processing play a larger role, suggesting that this hypothesis cannot fully explain the current data. Past research has shown enhanced cognitive benefits of arousal reappraisal in math exams (Jamieson et al., 2016), quantitative portions of standardized tests (Jamieson et al., 2010), and public speaking tasks (Beltzer et al., 2014). It is possible that my participants did not have pre-existing negative experiences and appraisals of these familiar contexts (e.g., test anxiety), that participants in other studies had. Of note, arousal reappraisal interventions have not shown cognitive benefits on other types of tasks, including qualitative sections of standardized tests (Jamieson et al., 2010) or math performance competitions against another individual (Hangen et al., 2019). 


\section{Limitations and Future Directions}

There were several limitations to this initial investigation into using an arousal reappraisal intervention with a memory performance task. A broader range of physiological measures, including cortisol levels and cardiovascular responses, would provide deeper insight into the results. First, assessing cortisol levels would confirm HPA axis activation, and allow for comparisons between conditions, as arousal reappraisal participants may show more moderate HPA activation than placebo participants (e.g., Jamieson et al., 2018; Jentsch \& Wolf, 2020; Mendes \& Park, 2014, but see Denson et al., 2014). Additionally, cortisol measures would allow separate examinations of high cortisol responders versus low cortisol responders. This comparison could reveal interesting effects, as some research on retrieval stress and memory suggests that impairing effects are only observed in high cortisol responders (e.g., Buchanan et al., 2006; Buchanan \& Tranel, 2008; Schönfeld et al., 2014). In future work, it may also be beneficial to explore the role of individual differences in subjective stress responding and coping (e.g., levels of social anxiety; Jamieson et al., 2013b). Second, although measures of blood pressure confirmed physiological arousal, blood pressure is an ambiguous measure in terms of determining challenge or threat states (Seery, 2011). Comparing conditions on cardiovascular responses (e.g., heart rate, vascular conductance, cardiac output, and total peripheral resistance) would provide better insight into the challenge versus threat states from a physiological perspective, rather than solely relying on self-reported affect and stress appraisals.

Some recent research also suggests sex effects, such that arousal reappraisal may improve physiological functioning for males but not for females (Hangen et al., 2019). Sex has also been shown to influence the relationship between retrieval stress and memory (e.g., Cahill et al., 2012), such that males often show higher cortisol responses to stress than females (e.g., Hidalgo et al., 2019). Hormonal contraceptive use and menstrual cycle also play a role in the stress response (e.g., Kirschbaum et al., 1999; Shields et al., 2017). Female participants using hormonal contraceptives can exhibit blunted stress hormone responses (e.g., Nielsen et al., 2013), leading some experiments to only include females who do not use hormonal contraceptives (e.g., Jentsch \& Wolf, 2020). My sample included mostly females $(81.92 \%)$, half of whom were using hormonal contraceptives and half of whom were not. Although I partially controlled for variations in sex and hormonal contraceptive usage by balancing these demographic factors across conditions, it is possible that the null effects stem from differences in sex. Data from the current experiment do not allow for a sufficiently powered analysis of potential sex differences, but future studies could treat sex as a factor in the design to allow for a well-powered comparison. 
Clearer task instructions for participants may benefit future research in a few ways. First, although participants were instructed to answer the stress reappraisal questionnaire for the task as a whole (i.e., including the MAST and memory test), it is possible that the vague terminology of "the task" in the questionnaire may have confused some participants. This does not appear to have been a problem, as participants show no differences at baseline, but only after they found out to which stress condition they were randomly assigned. Still, future research using this questionnaire could use more precise language to indicate to participants exactly which task they should assess when reporting their levels of task demands and coping resources. Second, free recall responses for eighteen participants were excluded because they completed one of the free recall questions for the wrong scene, did not use their native language, or did not provide a free recall response for one of the images. Memory tasks in future experiments could use a singular to-be-remembered item or a more specific prompt to avoid such confusion.

Some of the data in this experiment also suggest the possibility of a floor effect. Floor effects may make it difficult to observe potential effects of stress and reappraisal on memory performance, as they indicate that participants are performing at very low levels. In the current study specifically, the $d^{\prime}$ 'scores, which are around 0, suggest possible floor events for the recognition data. Such floor effects could obscure observation of the predicted negative effects of retrieval stress on sensitivity. These $d$ 'values likely stem from the fact that participants were required to make a choice for each recognition multiple choice question thereby increasing false alarm rates. However, the accuracy data do not indicate floor effects, with participants performing well above chance levels. Task difficulty likely played a role in this pattern of results. . I chose to use pictures as the stimuli as prior experiments investigating retrieval stress effects on memory have used pictures before (e.g., Hidalgo et al., 2015). However, the images used in the current study were more complex scenes. Additionally, past research has used these materials with very short retention intervals (i.e., 10 seconds; Sauer $\&$ Hope, 2016). I used these stimuli with a 24hour retention interval to better reflect eyewitness settings, where retention intervals are oftentimes lengthy and experiences complex. Future research might find different results with shorter retention intervals or a less demanding memory task, particularly in regard to measures of sensitivity. That being said, mirroring real world settings is important, and thus using even more realistic stimuli (e.g., mock crime, actual encounter with a target person) would be valuable for increasing ecological validity. 
Finally, it is possible is that effects of retrieval stress on memory are small rather than medium. I deliberately based the a priori power analysis on substantial previous relevant work suggesting medium effect sizes of retrieval stress effects on memory performance (e.g., Domes et al., 2004; Hidalgo et al., 2015; Kuhlmann et al., 2005; Schwabe \& Wolf, 2009; Schwabe \& Wolf, 2014, Experiment 2; Smeets, 2011; Tollenaar et al., 2009). Still, future work should aim to replicate the current findings, perhaps with larger sample sizes to capture even small effects of retrieval stress on memory performance.

There are several interesting future avenues for this line of research. First, examining the efficacy of stress mindsets (i.e., more broadly instructing participants that stress generally enhances ability) on similar stressors could elucidate whether arousal reappraisal is limited to certain types of stressors (i.e., social/evaluative vs. pain, etc.). Second, future experiments could examine memory using other stressful scenarios, where the stress induction is directly relevant to the memory task. For example, in one stress study, participants encoded neutral and emotional pictures and words (Schönfeld et al., 2014). Twenty-four hours later, they encountered either a stressful, oral examination test or a non-stressful free recall test. Using a stressful oral examination aligns more closely with past research on arousal reappraisal (i.e., exam performance, public speaking tasks, etc.), and understanding the efficacy of arousal reappraisal in such a setting would be relevant for certain educational settings (e.g., oral exams, presentations). In addition, such an approach would be a building block for the forensic field, more closely reflecting applied police interviews for witnesses. Third, research could directly compare cognitive tasks already demonstrated to be enhanced by arousal reappraisal (e.g., math questions) with motivated memory tasks to examine potential boundaries of arousal reappraisal effects. That is, a direct comparison would allow for a more informed discussion regarding the scope of the effects of arousal reappraisal on distinctive types of cognitive tasks. Finally, in the exploratory analyses, I found that the Stress-intervention condition showed a more conservative response bias than the No-stress-placebo control condition. Though no past research has directly examined the effects of acute retrieval stress and cognitive appraisals on response bias, this exploratory finding highlights a route for some interesting future research. For example, future experiments might more closely investigate how arousal reappraisal interventions alter individuals' decision criterions, particularly while experiencing stress prior to or during retrieval. 


\section{Conclusion}

In conclusion, I found no support for the hypotheses, as acute stress experienced prior to retrieval did not impair recognition and recall memory. The arousal reappraisal intervention also did not affect memory performance. In light of these findings, I can neither make conclusive statements about the effects of retrieval stress on memory performance beyond the data, nor definitively judge the efficacy of the intervention in all contexts of acute stress and memory performance. Still, given the body of research suggesting negative effects of retrieval stress on memory performance (e.g., Shields et al., 2017; Wolf, 2017), it is important for future research to continue exploring ways to mitigate negative effects of stress, both in terms of cognitive benefits but also in relation to emotional distress and coping ability. 


\section{CHAPTER 5}

\section{General Discussion}

This chapter draws from the following manuscript:

Marr, C., Sauerland, M., Otgaar, H., Quaedflieg, C.W.E.M., \& Hope, L. (2021). The effects of acute stress on eyewitness memory: An integrative review for eyewitness researchers. Memory, 29(8), 1091-1100. https://doi.org/10.1080/09658211.2021.1955935 
The central aim of this thesis was to examine the effects of acute stress at encoding and retrieval on eyewitness memory performance. To date, the research literature on the effects of acute stress on memory reports mixed findings. Eyewitness memory experiments often show impairing effects of stress at encoding, while some fundamental memory experiments demonstrate that encoding stress can enhance memory performance. Experiments examining retrieval stress also report mixed results, and little past work examined retrieval stress effects on memory in eyewitness contexts. In the program of research conducted for this thesis, I first surveyed eyewitness and fundamental memory experts and laypeople to understand current beliefs about acute stress effects on (eyewitness) memory (Chapter 2). In three subsequent laboratory experiments, I combined theoretical and methodological strengths of the eyewitness and fundamental memory fields to empirically advance understanding of the topic. Specifically, as presented in Chapter 3, two experiments using contemporary methodology were conducted to examine encoding and retrieval stress effects on face recognition performance. I next investigated retrieval stress effects on recognition and free recall memory performance, while also testing the efficacy of an arousal reappraisal intervention to potentially mitigate stress effects on memory (Chapter 4).

\section{Summary of the Findings}

The program of research conducted for this thesis started with an investigation into what current eyewitness memory experts, fundamental memory experts, and laypeople believe about different aspects of the relationship between acute stress and memory performance. Previous work on this topic was limited. Past surveys have typically used only a singleitem statement to assess belief about how stress affects eyewitness performance, namely Very high levels of stress impair the accuracy of eyewitness testimony (Kassin et al., 1989, 2001; Yarmey \& Jones, 1983). Not only does lack a specification of memory phase, but the surveys using this generic statement were conducted nearly twenty years ago, and a body of new research on this topic has since emerged (e.g., all experiments in Shields et al., 2017, meta-analysis). Results from the survey reported in Chapter 2 showed that a large proportion of both eyewitness and fundamental memory experts agreed that retrieval stress impairs memory performance but revealed a discrepancy between experts regarding encoding stress. Specifically, most fundamental memory experts agreed that encoding stress enhances memory, but most eyewitness experts disagreed with this statement. Additionally, laypeople's beliefs contrasted with those from experts in some key areas. For example, a majority of laypeople believed that police officers' memories are less affected by stress than other witnesses, and that stress can cause repressed memories, 
yet neither beliefs are supported by experts and both conflict with empirical findings (e.g., Stanny \& Johnson, 2000; Otgaar et al., 2019). Results reported in this chapter highlighted areas of consensus and divergence between and within research fields, as well as within the broader population. These survey results regarding retrieval stress effects on memory guided the methodology in Experiment 2, reported in Chapter 3, and in Experiment 4, reported in Chapter 4.

The second aim of this thesis was to experimentally investigate these effects in laboratory settings relevant to the eyewitness context of face recognition. In Experiments 2 and 3 (Chapter 3), I used contemporary methodology from the fundamental memory field, using a verified stressor (i.e., MAST), confirming the acute stress induction physiologically (i.e., blood pressure and salivary cortisol) as well as subjectively, and implementing a 24hour retention interval to isolate encoding effects from retrieval effects. These experiments used stimuli relevant to the eyewitness field (i.e., faces) and examined encoding of these faces both during (i.e., as an integral part of the stressor) and after the stressor (i.e., during the cortisol peak). Across both experiments, the stress manipulations were successful, with participants in the stress condition showing increased negative affect, blood pressure, and cortisol levels (in Experiment 3) compared to those in the control conditions. In Experiment 2, the predicted effects of acute stress during neither encoding nor retrieval on face recognition memory were found. To address this unexpected finding, I investigated encoding stress effects on memory performance again in Experiment 3. In addition to face stimuli, I included word stimuli on this occasion. Words are a more commonly used stimulus in fundamental memory research and past studies using words have displayed stress effects on memory (e.g., Schwabe et al., 2008a; Zoladz et al., 2011). Yet, I found no effect of acute stress on memory for either stimulus type. In sum, in these two experiments, findings demonstrate that acute stress neither affected face recognition memory performance nor word recognition performance for encoding stress.

In Chapter 4, I was interested in understanding the effects of retrieval stress on memory performance in more detail and testing a technique for reducing potential negative effects of stress on memory. Specifically, I explored (1) the effects of retrieval stress on recall and recognition of a complex image, and (2) the efficacy of an arousal reappraisal intervention to mitigate any negative effects of stress on memory performance in Experiment 4. Again, the stress manipulations were confirmed with stressed participants showing increased negative affect and blood pressure compared to control participants. However, retrieval stress had no effect on memory performance and arousal reappraisal had no effect on 
memory performance or affect, task demands, or coping resources. Thus, the results of Experiment 4 broadly suggested an absence of a reliable effect of acute retrieval stress or arousal reappraisal on recall and recognition memory performance.

\section{Effects of Acute Stress at Encoding on Memory: Integrating the Current Findings}

Results from the survey reported in Chapter 2 showed consensus between experts on some factors but demonstrated a lack of consensus on several important areas in the field of stress and memory. For example, high levels of consensus were found for the statement Very high levels of stress impair the accuracy of eyewitness testimony, with the majority of experts and laypeople agreeing with the statement. However, though the majority of fundamental memory experts also agreed that Experiencing stress during an event enhances memory for that event, the majority of eyewitness experts disagreed. These diverging beliefs reflect mixed findings within each field, with eyewitness research more often reporting impairing effects of encoding stress on memory performance (e.g., Deffenbacher et al., 2004), and fundamental research more often reporting enhancing effects (e.g., Shields et al., 2017). They also align with agreement levels given by each expert group on related neuroscientific statements about encoding stress and memory. Specifically, fundamental experts more commonly endorsed the idea that ANS activity and rapid cortisol facilitate memory encoding than eyewitness experts. Such differences in understanding of underlying neurobiological processes may help explain the contrasting responses on the broader statement. Furthermore, these divergent beliefs likely reflect a lack of interaction between researchers in the different fields. Thus, results reported in this survey highlight the need for shared knowledge between the fields, ideally leading to collaborations between researchers in each area.

Other statements in the survey reflected past stress-memory models and provided varying levels of support for different stress-memory models. Specifically, majorities of both expert groups agreed that eyewitnesses who experience moderate stress during encoding show enhanced memory performance and that severe stress harms memory but that moderate stress does not. These beliefs resemble the inverted U-relationship of the YerkesDodson law, which proposes enhanced performance at medium levels of arousal, but impaired performance at low or high levels (Yerkes \& Dodson, 1908). Laypeople, on the other hand, showed lower levels of endorsement for these statements, suggesting more general commonsense beliefs regarding the negative effects of encoding stress on memory 
performance. Another statement asked about detail type: Stress experienced during an event (i.e., at encoding) enhances memory for central details of the event, but not for peripheral details. This statement reflects an important element of Easterbrook's cue utilization hypothesis, which predicts that attentional narrowing may result in enhanced memory for central details but impaired memory for peripheral details. A majority of experts from both fields agreed with this statement, though a majority of laypeople did not share this belief. Finally, especially eyewitness experts mostly selected don't know in response to neuroscientific statements relating to current stress-memory models, though don't know responses on these statements were also fairly high among fundamental experts. Such responses suggest a lack of widespread knowledge or support for newer models regarding effects of encoding stress on memory performance.

The null findings reported in Chapter $\mathbf{3}$ do not support the current models of stress and memory. For example, the dual-mode model and temporal dynamic model suggest that stress immediately prior to or during encoding enhances memory performance (de Kloet et al., 1999; Diamond et al., 2007; Joëls et al., 2006; Schwabe et al., 2012). In Experiments 2 and 3, I examined two distinct stages (i.e., during and after the stressor) to observe the role that stressor timing may play. However, I found no memory enhancements, even for materials encoded during the stressor, when stress and learning converge and the memory formation mode is active. In both experiments, I also intentionally integrated the stimuli into the stressor. Specifically, the face encoding during the acute stress phase was part of the stressor, with participants engaging in alternating blocks of hand immersion into ice cold water, socially evaluated mental arithmetic, and face encoding. I made this methodological decision because the dual-mode model and temporal dynamic model suggest stronger effects for stressor-relevant materials (Joëls et al., 2006; Schwabe et al., 2012). Similarly, although the executive control model generally posits negative effects of encoding stress on memory performance, it suggests that stress during encoding should facilitate encoding when information is integral to the stressor (Gagnon \& Wagner, 2016; Mather \& Sutherland, 2011). Yet, despite the face encoding being part of the stressful experience, results showed no statistically significant effects of acute encoding stress on face recognition memory.

These results are inconsistent with many past empirical findings. Regarding faces, one previous fundamental memory experiment showed memory enhancements for faces encoding during a laboratory stressor (Wiemers et al., 2013). Previous eyewitness research suggested negative effects of acute encoding stress on face recognition performance (Davis 
et al., 2019; Deffenbacher et al., 2004; Pezdek et al., 2020), although differences in methodology likely contribute to these discrepant results. However, of crucial importance for the current thesis, another eyewitness memory experiment used similarly robust methodology_-including a MAST stress induction, verifying stress with cortisol measures, and applying a sufficient retention interval—-found no effects of acute encoding stress on lineup identification performance (Sauerland et al., 2016). Therefore, results reported in this thesis do align with some past work and perhaps confirm that when contemporary methodology is used to induce and confirm stress (e.g., Shields et al., 2020), encoding stress effects on eyewitness memory are not reliable.

Results from Experiment 3 additionally showed that acute encoding stress experienced during and prior to encoding also did not affect word recognition memory. These findings are unexpected given the ample literature supporting enhancements for word recognition (e.g., Abercrombie et al., 2003; Domes et al., 2002; Schwabe et al., 2008a; Zoladz et al., 2011). That being said, many experiments that have reported enhancing effects of encoding stress on word recognition have done so only under certain conditions. For example, one experiment suggested enhancing effects for high cortisol responders, but not for all participants who experienced encoding stress (e.g., Domes et al., 2002). I conducted a similar exploratory analysis, but even when comparing high and low cortisol responders separately, results still showed no statistically significant effects of encoding stress on word recognition performance. Other previous experiments reporting memory enhancements have shown such enhancements for only positively valenced, but not neutral or negative words (e.g., Zoladz et al., 2011), or neutral words, but not positive or negative words (e.g., Schwabe et al., 2008a). The words used in Experiment 3 were negatively valenced (e.g., abuse, punishment, etc.), so perhaps these variations in valence help explain the contrasting findings. Furthermore, other experiments have observed null effects when examining acute encoding stress effects or cortisone administration on word recognition performance (de Quervain et al., 2000; Domes et al., 2004). Thus, the findings from Experiment 3 are not solely inconsistent with past work. It is worth noting that a recent preprint with meta-analytic findings shows evidence that pre-learning psychosocial stress does not have a statistically significant effect on memory for either short (Cohen's $d=-0.02$ ) or long (Cohen's $d=0.25$ ) retention intervals (McManus et al., 2020). These findings, combined with findings reported in this thesis, add further uncertainty to the robustness of encoding stress effects on memory performance.

In sum, the results reported in Chapter $\mathbf{2}$ and $\mathbf{3}$ show a lack of consensus between experts 
regarding the effect of encoding stress on memory performance and provide some new evidence indicating that mild to medium levels of acute stress do not necessarily affect memory performance. Still, when integrating these findings within the broader literature, it is apparent that empirical research on this topic does not yet provide a conclusive answer. Furthermore, based on the null results from Experiments 2 and 3, which used contemporary methodology to examine the relationship between acute encoding stress and face recognition memory, caution is appropriate when applying past findings in legal settings, as previous experimental findings may be constrained to specific samples, manipulations, situations, or stressors. As such, the results in this thesis also highlight the ongoing need for further robust research on the relationship between acute stress and memory in legal settings.

\section{Effects of Acute Stress at Retrieval on Memory: Integrating the Current Findings}

In Experiment 1, reported in Chapter 2, I sought to understand what current memory experts and laypeople believe about retrieval stress effects on (eyewitness) memory performance and potential related moderators. In Experiment 2, reported in Chapter 3, I examined how stress experienced during and prior to retrieval affected face recognition performance. Finally, in Experiment 4, reported in Chapter 4, I investigated acute retrieval stress effects on recognition and recall memory for a complex scene and tested the efficacy of a cognitive reappraisal intervention to potentially reduce negative effects of stress on memory.

Responses to the survey, as presented in Chapter 2, paint a picture of broad consensus across experts and laypeople about beliefs about the general effects of acute retrieval stress on memory performance. Specifically, large majorities of the three groups sampled agreed that Experiencing stress while trying to remember something impairs memory retrieval. Slightly lower proportions but still large majorities of each group also endorsed a more applied version of this statement, suggesting general agreement that if an eyewitness is stressed during a police interview, their memory will be less accurate than if he or she were not stressed. These reported levels of agreement align well with current neurobiological models of stress and memory, such as the dual-mode model, the temporal dynamics model, and the executive control model, which all similarly suggest general impairing effects of retrieval stress on memory performance. Similarly, results are in line with many fundamental research findings on this topic (e.g., Het et al., 2005; Shields et al., 2017; Wolf, 2009, 2017). 
The survey reported in Chapter 2 also included statements about more specific factors that may moderate the retrieval stress-memory relationship. First, a majority of experts from both disciplines and laypeople agreed on differential effects of retrieval stress for free recall ability rather than recognition ability, a view that is reflected in the literature (e.g., Gagnon \& Wagner, 2016). Second, only small proportions of each group agreed that If memory is immediately tested after a stressor, one does not experience a memory deficit; rather, memory at this stage can actually be enhanced. Thus, although a growing body of research suggests that stress experienced during or immediately prior to retrieval does not affect or can even enhance memory performance (e.g., Hupbach \& Fieman, 2012; Schönfeld et al., 2014; Schwabe \& Wolf, 2014; Smith et al., 2016), general support from experts is lacking on this statement. Finally, the neurobiological mechanisms underlying the impairing effects of retrieval stress also appear to require further understanding. There were low levels of agreement in both expert groups that memory is impaired when noradrenergic arousal interacts with non-genomic glucocorticoids during retrieval. Though this is the mechanism that models such as the dual-mode model propose regarding stress at retrieval, $87 \%$ of eyewitness experts and $44 \%$ of fundamental memory experts still selected don't know. These survey results regarding retrieval stress effects on memory helped guide further investigation in Experiment 2, reported in Chapter 3, where I examined distinct stages of retrieval (i.e., during stressor vs. post-stressor). The generally accepted negative effects of retrieval stress by both expert groups also guided the methodology in Experiment 4, reported in Chapter 4, where I examined the efficacy of a potential intervention to mitigate such retrieval stress impairments and examined both recognition and recall performance.

As presented in Chapter 3, results from Experiment 2 showed no statistically significant effects of acute retrieval stress on face recognition memory performance. These findings do not align with current models of stress and memory, which uniformly suggest that acute retrieval stress impairs memory (e.g., de Kloet et al., 1999; Diamond et al., 2007; Joëls et al., 2006; Schwabe et al., 2012). Still, these models focus on the importance of the interaction between ANS and HPA axis activity; this interaction would only have taken place during the post-stressor stage. Indeed, some empirical research supports the idea that with little or no delay between stressor and retrieval, acute retrieval stress may not affect or may even enhance memory performance (e.g., Hupbach \& Fieman, 2012; Schwabe \& Wolf, 2014; Smith et al., 2016). However, Experiment 2 revealed no effect of retrieval stress on face recognition performance at either stage (i.e., during stressor and post-stressor). Very limited work has specifically examined retrieval stress effects on 
face recognition memory. Two of the experiments that have examined this topic showed discrepant findings: one experiment suggested impairments for face recognition sensitivity (Li et al., 2013), but the other published work and two unpublished doctoral theses suggested no statistically significant effects of acute retrieval stress on face recognition memory performance (Dellapaolera, 2019; Li et al., 2014; Robicheaux, 2016). Findings regarding retrieval stress reported in Chapter 3 add to this small body of research, providing more data that suggest an absence of acute stress effects during or prior to retrieval on face recognition performance, and questioning the broad generalization that retrieval stress reliably impairs memory (e.g., Smith et al., 2016; Wolf, 2017).

Results reported in Chapter $\mathbf{4}$ similarly showed an absence of statistically significant effects of retrieval stress on memory performance. In Experiment 4, I induced stress prior to assessing both recall and recognition memory for a complex stimulus image. Strikingly, retrieval stress did not affect either test type. These results are inconsistent with a substantial body of past work regarding retrieval stress effects on memory performance, particularly on recall performance where stress effects are generally stronger (de Quervain et al., 2000, 2003; Gagnon \& Wagner, 2016; Wolf, 2017). Certain moderating factors may help explain these null results in Experiment 4. For example, meta-analytic findings suggest that acute retrieval stress negatively impairs memory to a larger degree for negative than for neutral items (Shields et al., 2017). Although the images were mildly negatively valanced, they were not extremely emotional or distressing. Additionally, meta-analytic results suggest that including women taking hormonal contraceptives rendered the effect of retrieval stress on memory negligible. My study included men, women taking hormonal contraceptives, and women not taking hormonal contraceptives. This methodological difference likely contributed to the null findings, crucially revealing that retrieval stress effects on memory are likely more constrained and fragile than is often explicitly stated in reviews or believed by experts (e.g., Marr et al., 2020; Shields et al., 2017; Wolf, 2017).

Another key research question described in Chapter $\mathbf{4}$ was an examination of the efficacy of an arousal reappraisal intervention, given that impairing stress effects at retrieval on memory are generally widely found. Such an intervention could be useful in legal settings, where, for example, police officers could provide stressed individuals with techniques to both improve their well-being and reduce potential memory impairments. Past research suggests that in situations where acute stress cannot be avoided, people can employ a learnable cognitive reappraisal tactic to reframe how they view the stress that they experience (e.g., Beltzer et al., 2014; Jamieson et al., 2010, 2016, 2018; Mendes 
\& Park, 2014). Thus, I examined the efficacy of an arousal reappraisal intervention on a memory task to observe potential benefits on performance. As no impairing effects of retrieval stress on memory performance were observed, it is not possible to precisely say whether arousal reappraisal reduces such stress effects. Still, I investigated possible group differences in memory performance between participants who received the intervention and those who received the placebo. Results from Experiment 4 showed no effects of the intervention on memory performance, on affect, or on appraisals of task demands and coping resources. These findings provide an initial example of arousal reappraisal being used in situations of motivated memory performance and results do not indicate that the intervention was a success in regard to improving coping ability or memory performance. However, Experiment 4 is the first to examine an arousal reappraisal intervention in such a setting, and thus, these findings need to be replicated before definitive conclusions can be drawn.

In sum, results reported in Chapter 2, 3, and $\mathbf{4}$ show that although effects of retrieval stress on memory performance are more widely accepted by experts, these effects may be more limited than often presented. Specifically, in Experiments 2 and 4, retrieval stress neither reliably impaired recognition nor recall. Though inconsistent with past work (e.g., Shields et al., 2017; Wolf et al., 2017), findings reported in this thesis are consistent with other recent meta-analytic (still unpublished) results, which showed a lack of statistically significant effects of psychosocial retrieval stress on memory performance (Cohen's $d=-0.23$; McManus et al., 2020). These recent findings highlight the importance of examining relevant moderators of the stress-memory relationship, which suggest that even minor differences between research designs can alter the effects that acute retrieval stress has on memory. As such, these findings show the need for further research on this topic to discover the limits of possible impairing effects of retrieval stress on memory performance, especially in situations that resemble applied legal settings. Additionally, it would be valuable to continue further explorations of possible interventions to improve memory performance in stressful situations. 


\section{Implications of this Research}

\section{What are the Theoretical Implications of Findings Reported in this Thesis?}

Cumulatively, the results from this thesis do not lend support to the contemporary stress-memory models, such as the temporal dynamics model and the dual-mode model. Specifically, null findings were observed despite confirmed activation of the autonomic nervous system and HPA axis using objective physiological measures, and examinations across different stages of stressor timing (i.e., acute stress stage vs. delayed stage) and varying levels of task difficulty (i.e., recognition vs. free recall, highlighted by Diamond et al., 2007 as a key factor). As such, these findings lead to further questions about the models.

There are a few main factors to consider when comparing results from this thesis to predictions from the stress-memory models. First, it is important to note that the development and initial evidence for these models were based on experiments performed on non-human animals, such as rats (e.g., Diamond et al., 2007; Joëls et al., 2006). Diamond and colleagues (2007) explicitly highlighted the near exclusive use of samples of male rats and state that results may not equally apply to female rats, which also brings into question the ease of generalizability to male and female humans. Second, given that these neurobiological models are based on physiological reactions to acute stress (e.g., increased noradrenaline, glucocorticoids, etc.) in various brain areas (e.g., amygdala, hippocampus, PFC, etc.), the authors state that the magnitude and duration of the stressors play a large role in subsequent effects on memory. However, the types of stressors used in these animal experiments, such as examining water maze memory in rats, greatly varies from stressors used in human studies where there are stricter ethical limitations. As such, the models may not easily translate to humans and human studies of stress and cognition without clearer indicators outlining distinct levels of stress severity. For example, though I used physiological measures to confirm stress inductions, perhaps the levels of stress induced do not align with those assumed by the models, an element which is currently vague. Third, as mentioned in Chapter 1, results from some human experiments support elements of the stress-memory models, such as those showing distinctions in memory performance between the memory formation mode and memory storage mode (e.g., Quaedflieg et al., 2013; Schwabe \& Wolf, 2014; Zoladz et al., 2011). However, other research suggests the ongoing complexity of understanding the intricacies of stressor timing within the context of these models (Quaedflieg \& Schwabe, 2018), with some findings not adhering to the 
predictions laid out by the models (e.g., a lack of memory storage mode impairments in Vogel \& Schwabe, 2016). Although my experiments followed the anticipated timing of physiological stress responses, perhaps slight variations in the study designs would have produced different results. Finally, the importance of stressor relevance of the to-beremembered stimuli is highlighted by the dual-mode model. The stimuli in my experiments may not have been considered to be stress-relevant by the participants and this element may provide an additional possibility of why the results from this thesis do not align with the stress-memory models.

The data in this thesis suggest that the stress-memory models may not be widely generalizable or are based on small effect sizes that are difficult to detect in measures of human memory. Notably, results described this thesis also reveal a lack of knowledge of these contemporary stress-memory models by individuals identifying as memory experts. These results further emphasize the importance of gathering additional empirical evidence. Also apparent is the need for increased interdisciplinary engagement between eyewitness and fundamental memory researchers. Researchers from each field should be knowledgeable of the goals, methodologies, and findings reported in the other research field. Ideally, collaborations between researchers from the distinct fields would produce methodologically strong research that better reflects real world conditions.

The null findings described in Chapter $\mathbf{3}$ and $\mathbf{4}$ also have theoretical implications beyond those directly related to the contemporary stress-memory models. Notably, I used power analyses to ensure sufficient sample sizes and combined methodology from the fundamental field and eyewitness memory field by using validated stressors, manipulation checks, sufficient retention intervals, and eyewitness-relevant stressor timing and stimuli. These null findings suggest that the effects of acute stress on memory performance may not be as strong as other studies have suggested.

As such, the null findings reported in this thesis bring into question the generalizability of past findings regarding acute stress effects on memory performance. As highlighted throughout this thesis, many eyewitness experiments on this topic have significant methodological limitations, such as not validating acute stress inductions or ensuring memory phase distinctions, making it difficult to specifically draw conclusions about acute stress effects on memory performance. Although the fundamental field effectively confirms stress inductions, many statistically significant findings are also moderated by other factors. These factors may strongly narrow the boundaries of observed effects of 
acute stress on memory performance, a particularly important element when considering applied implications for legal settings. First, using very basic stimuli such as word lists may reduce effects of stress on attention, which likely plays a larger role in eyewitness settings in the real world (e.g., Hyman et al., 2018; Sammon \& Bogue, 2015). Second, several studies have suggested enhanced or impaired memory performance only in subsets of stressed participants, for example high cortisol responders (e.g., Buchanan et al., 2006; Buchanan \& Tranel, 2008; Domes et al., 2002; Schönfeld et al., 2014). Third, to better isolate for stress effects and control for variations in cortisol, the fundamental field typically uses strict inclusion criteria, like only recruiting healthy participants with a restricted BMI who are not on certain medications or participating in psychological treatment (Shields et al., 2020). Further, many of these studies recruit or observe statistically significant effects only in narrow portions of the population, for example in men (e.g., Andreano \& Cahill, 2006; Kuhlmann et., 2005b) or women not using hormonal birth control (e.g., Nielsen et al., 2013; Vogel \& Schwabe, 2016a; Wiemers et al., 2013). Using such limited samples excludes large parts of the general young adult population: around of half the human population are females and around a quarter of the fertile female population uses hormonal birth control in the United States (Daniels \& Abma, 2020). Sometimes women are tested only at certain times of their menstrual cycle (e.g., Maki et al., 2015; Schoofs $\&$ Wolf, 2009). Additionally, most research is only conducted in the afternoon to control for diurnal cortisol cycles (e.g., Schönfeld et al., 2014; Schwabe et al., 2008a; Schwabe \& Wolf, 2014; Vogel \& Schwabe, 2016a). These strictly controlled elements allow for stronger stress effects and more precise interpretations (Shields et al., 2020). However, the broader generalizability of such research is greatly reduced. For example, if statistically significant effects of acute stress on memory are only observed in the afternoon, in healthy men who are high cortisol responders at $3 \mathrm{pm}$, how can we then practically apply this knowledge to eyewitness contexts? Research pursuing more fundamental questions of when and how stress affects memory is important and should continue, but there also is a need for research permitting broader generalizations.

\section{What are the Practical Implications of Findings Reported in this Thesis?}

Some practical implications can be drawn from the results reported in this thesis. To begin with, results reported in Chapter 2 highlight the importance of beliefs held by memory experts and laypeople. What these groups believe about the effects of acute stress 
on memory performance can enter the courtroom. For example, memory experts may testify in court, as $29 \%$ of the expert sample in Experiment 1 had previously done. The survey results suggest that experts do not agree about how encoding stress may affect memory performance, and thus different experts may communicate different opinions in the courtroom. In this way, jurors and judges could hear contrasting statements from opposing expert witnesses, or hear from only one expert witness, who could be a proponent of one particular perspective - situations like this do sometimes occur in legal settings (e.g., Brackmann et al., 2016). Either way, this presents a problem: jurors could accept or discount eyewitness testimony depending on which expert they hear from, or which expert they trust more. Thus, for statements that show lower levels of expert consensus, judges and other legal practitioners should exercise caution when deciding whether or not to allow expert witness testimony. On the other hand, if expert witnesses are nuanced in their presentation of these complex issues or share evidence supporting more solidly empirically supported statements, jurors may benefit greatly from such expert testimony.

Including expert testimony may particularly be valuable for certain commonsense beliefs that seem to be held strongly by laypeople. For example, it is useful to know that laypeople assume that any amount of stress will automatically impair memory. This belief could later affect their legal decisions, for example by viewing the testimony from a stressed eyewitness as lacking in probative value (e.g., Bornstein et al., 2008). Additionally, laypeople may give too much credence to police testimonies over other eyewitness testimonies if they believe that police are less affected by stress. In such circumstances, expert testimony may help combat these preconceived beliefs, educating jurors and allowing them to make more sound, empirically based decisions about how to interpret eyewitness evidence.

The limitations of past findings paired with the null results in the current findings and in other recent research (e.g., Sauerland et al., 2016) raise questions about how well the current state of the field can inform practice. Findings reported in Chapter $\mathbf{2}$ and $\mathbf{3}$ of this thesis suggest continued uncertainty regarding the effects of mild to medium levels of acute stress on face recognition performance. Past research, as well as greater levels of expert consensus reported in Chapter 2, provide stronger evidence for impairing effects of acute retrieval stress on memory performance. However, results reported in Chapter 3 and $\mathbf{4}$ suggest that even these effects do not always emerge. Therefore, at the moment, the line of research presented in this thesis does not provide an unambiguous answer to the broader question of how acute encoding and retrieval stress affects eyewitness memory performance. Instead, I emphasize methodological suggestions for future researchers with 
the goal of producing a more reliable and generalizable body of research on this topic. However, before doing so, I will first outline some of the methodological limitations present in the current thesis.

\section{Methodological Limitations}

This thesis aimed to combine the strengths of the eyewitness and fundamental research fields to apply robust methodology to examine the effects of acute stress on memory performance in eyewitness contests. Nonetheless, the experiments in thesis also have several important methodological limitations. First, this thesis aimed to examine the stress-memory relationship with an applied eyewitness focus. Unsurprisingly, ethical considerations restrict the severity of the stress that participants can experience in laboratory settings. I chose to investigate stress effects in laboratory settings to allow for internal validity so that I could draw causal conclusions. However, this also means that stress levels in this thesis are classified as mild to medium levels of stress, rather than severe stress. Mild to medium levels of stress are likely experienced by many eyewitnesses in less severe crimes. However, the stress experienced in the lab is not equivalent to, for example, experiencing an extremely violent or life-threatening crime. Indeed-though some research suggests that laboratory stressors produce similar physiological reactions to brief acute stressors in real life (e.g., Henze et al., 2016; Rajcani et al., 2016)—as Shilton and colleagues (2017) state in their paper examining the efficacy of the MAST, "many laboratory stress tests appear to better model moderate acute stress in daily life, rather than overwhelming acute trauma" (p. 2). Thus, discussions of stressed witnesses in this thesis are not akin to witnesses who have experienced trauma - the effect of extreme stress or trauma on witness memory is certainly another important relevant area of study. As such, the findings and implications discussed in this thesis are limited to stressful situations of lower severity.

Second, in real eyewitness encoding contexts, the to-be-remembered event and the stressor are the same (i.e., a crime). Research suggests that stressor relevance may moderate the relationship between encoding stress and memory, such that only information relevant to the stressor will be enhanced by stress at encoding (Joëls et al., 2006; Shields et al., 2017; Smeets et al., 2007; Vogel \& Schwabe, 2016a; Wiemers et al., 2013). To address this issue and still use a laboratory stressor to ensure a valid stress induction, I incorporated the stimuli into the stressor in the experiments reported in Chapter 3 to make the stimuli integral to the stressful experience. However, it is possible that participants did not view 
the stimuli portions of the MAST as inherent to the rest of the stressor, which may explain the lack of effect of encoding stress on face recognition performance. Previous studies have ensured stressor relevance by using a memory test specifically about the stressor itself (e.g., Vogel \& Schwabe, 2016a). Stressor relevance may also be relevant for retrieval contexts, because in real eyewitness contexts, the stressor and the retrieval are often aligned (e.g., as in a police interview). In Experiment 4, reported in Chapter 4, although the stressor and memory task were identified as one united task, it is possible that participants instead viewed them distinctly. Using a more intertwined retrieval stressor could overcome this limitation. For example, Schönfeld and colleagues (2014) used a stressful TSST-like oral examination to induce stress and test memory retrieval, an approach that also may better reflect an eyewitness' experience at a police interview or on the witness stand.

Third, each laboratory-based experiment was conducted in the Netherlands and investigated these research questions in a sample of consisting of mostly WEIRD (Western, educated, industrialized, rich, democratic; Henrich et al., 2010) participants. Experiments 2 and 3 were additionally limited by race, including only white participants and stimuli to reduce own-race bias (e.g., Meissner \& Brigham, 2001). Experiment 4 included native English, Dutch, or German speakers to allow participants to complete the memory task in their native language. All of these elements similarly reduce the generalizability of the research findings in this thesis. Before declaring enhancing or impairing effects of acute stress on memory performance, findings need to be replicated in more diverse groups of people. This includes people from non-WEIRD societies, people of different ages, non-student samples, and people of different races and ethnicities. For example, exploring race as a factor in the relationship between encoding stress and face recognition memory is crucial, as attention and cognitive control may be affected differently when encoding other race faces vs. same-race faces (e.g., Brown et al., 2017). Without replicating results in more diverse samples, it will remain difficult to give broadly adequate recommendations for applied settings.

Finally, null findings are ambiguous in that they do not establish that the null hypothesis is true, but rather merely fail to reject it. In my studies, I therefore applied Bayesian statistics where appropriate to gather more insight into null effects. For example, preregistered Bayesian analyses reported in Chapter 3 largely suggested substantial or strong evidence in favor of the null hypothesis, and pre-registered Bayesian analyses in Chapter 4 comparing the Stress-intervention group and the control group (i.e., No-stress-placebo group) showed substantial evidence for the null hypothesis. Still, replication is necessary 
to reinforce any null findings, as absence of evidence is not necessrily evidence of absence. Additionally, all my studies were also pre-registered and Experiment 4 was accepted as a registered report, meaning all hypotheses, sample sizes, and statistical analyses for each study were decided prior to data collection. However, 1) I based my power analyses on medium effect sizes, as suggested by most of the past research on acute stress and memory, and 2) I powered my experiments to $80 \%$ (Experiment 2 and 3) or 90\% (Experiment 4). Therefore, there is still a chance that these results show false negatives, or that smaller effects exist that were not captured with the sample sizes used in this thesis. Still, differences observed with smaller effect sizes may not be relevant in the real world. Police and legal practitioners are probably only interested in effects that are large enough to allow for reliable assertions about how stress has affected an eyewitness' memory performance in practice. Thus, when researching stress effects on eyewitness memory performance, medium or large effect sizes likely hold the most value.

It is also important to note that widespread use of open-science practices is fairly new in the psychological field. Therefore, previously published papers may be more prone to being underpowered or including questionable research practices, such as conducting numerous statistical analyses on the data, but only reporting the statistically significant results. Additionally, due to publication bias, experiments showing null results may have never been published. Indeed, in a meta-analysis on this topic, there was evidence for publication bias for experiments examining stress effects at encoding and retrieval on memory performance (Shields et al., 2017). Specifically, the analyses suggested that there was a disproportionate amount of impairing effects reported in these publications, compared with null or enhancing stress effects. This publication bias likely makes it difficult to compare the null findings in my experiments with similar findings that may exist in unpublished data.

\section{Future Research Directions and Recommendations}

This research broadly investigated several questions related to the effect of acute stress on eyewitness memory. Many future directions remain. For example, stress effects during encoding on face recognition memory should be studied further given the importance of this type of stimuli in eyewitness contexts. Examining effects on face recall memory would also provide a valuable advancement of the field. More attention should be given to how stress affects retrieval memory in more applied settings such as during stressful police interviews or when giving testimony. Future research should also develop or test other 
interventions to reduce any negative effects of retrieval stress on memory performance. More generally though, and perhaps more importantly, the work reviewed and conducted in this thesis provides an overview of methodological recommendations for future research in this area. Using contemporary, robust methodology will yield a strong collection of empirical studies, from which better and more reliable interpretations can be made.

Most critically, ensuring valid stress inductions is a prerequisite for effectively studying the effects of acute stress on memory performance. Researchers in the fundamental memory field have developed several laboratory stressors (e.g., TSST, MAST, etc.) that address this need, and eyewitness researchers can use such stressors knowing they are valid and reliable ways to induce acute stress. Still, it would also be possible for eyewitness researchers to use ecologically valid scenarios or field studies, as long as they still confirm sufficient stress inductions using subjective measures (e.g., negative affect), measures of ANS activity (e.g., blood pressure, heart rate, alpha-amylase), and measures of HPA axis activity (e.g., cortisol). Additionally, if the variable of interest is encoding stress, eyewitness memory researchers should use retention intervals of at least 24 hours to avoid the confounding effects of stress on retrieval processes as well as to better mimic the reality of investigations. Eyewitness researchers and fundamental memory researchers who would like to conduct more ecologically valid work should also consider stressor relevance, task type, and stimulus type. Specifically, I suggest that to understand the effects of stress on memory for eyewitnesses who become stressed due to the crime, the stressor and encoding process should occur simultaneously (i.e., experience stress during encoding). Also, the material encoded should stem from the stressor itself because (a) that is what happens in real life, and (b) stressor relevance moderates the relationship between acute encoding stress and memory performance (Joëls et al., 2006; Shields et al., 2017). As previously accomplished in some experiments (e.g., Vogel \& Schwabe, 2016a; Wiemers et al., 2013), this method can be accomplished by using a reliable stress induction (e.g., TSST, MAST, etc.) and testing memory for the stress induction itself or by adapting the stress induction to include the to-be-remembered elements within it (i.e., as part of the stressor). Alternatively, more naturalistic stressors could be used to ensure stressor relevance so long as acute stress is confirmed with appropriate biomarkers (e.g., cortisol), as highlighted above. Additionally and ideally, the task and stimuli should also be relevant to eyewitness scenarios (e.g., experiencing a crime, identifying a perpetrator, etc.) to further increase ecological validity. 
One recent experiment integrated methodologies from the two research fields by combining acute stress confirmed with objective physiological measures with an eyewitness scenario. Specifically, participants were exposed to an unanticipated mock theft just after the MAST (Sauerland et al., 2016). This methodology could be expanded, so that the to-be-remembered material originates from the stress induction. For example, experiments could stage a mock altercation between experimenters during the TSST. Additionally, objective physiological measures could be applied in high fidelity training scenarios, where stressors are more realistic. These types of developments would rapidly progress the state of applied research on this topic and provide a great example of linking the distinct strengths of the eyewitness and fundamental memory research fields.

Future experiments should also be designed with other potential moderating factors in mind. For example, encoding stress sometimes shows larger effects on memory when emotional material is used rather than neutral material (Goldfarb et al., 2019; Wiemers et al., 2019; Wolf, 2008, but see Shermohammed et al., 2019; Shields et al., 2017). Because the emotional valence is likely negative in applied settings, eyewitness researchers exploring stress effects may want to use emotionally negative materials (cf. neutral events) where possible. Other important moderating factors of stress and memory are biological sex and the time of day that testing takes place (e.g., Shields et al., 2017, 2020). As previously mentioned, these factors are often controlled for or examined in fundamental memory research, but not regularly in eyewitness research. Even if these are not the main research questions, researchers could aim to gather enough participants of each type to test for interactions between different sex hormone groups and stress on memory performance (Shields et al., 2020). At the very least, collecting data about these factors (e.g., hormonal contraceptive use, menstrual phase, etc.) and/or balancing gender and sex hormone status across experimental groups would be beneficial. Similarly, because stress hormones change throughout the day (e.g., Maheu et al., 2005), the time of testing should be considered. When investigating stress effects, it is vital for eyewitness researchers to understand how time of day could influence results, and empirically examine whether results in eyewitness settings are reliably found throughout the day. Finally, future research should also aim to better understand how acute stress affects factors like attention by using more complex scenarios (Wulff \& Thomas, 2021). However, ensuring that acute stress is properly induced and measured should always be a priority, as without this key element, conclusions about stress effects cannot be drawn. 
I conclude by reemphasizing that the results reported in this thesis cast doubt on the strength and generalizability of previous research findings. As such, legal practitioners should use caution when applying findings regarding acute stress effects on memory in eyewitness settings. These findings also highlight the need for further research using contemporary and robust methodology to improve understanding of the complex relationship between acute encoding and retrieval stress and memory performance. 


\section{References}

Abercrombie, H.C., Kalin, N.H., Thurow, M.E., Rosenkranz, M.A., \& Davidson, R.J. (2003). Cortisol variation in humans affects memory for emotionally laden and neutral information. Behavioral Neuroscience, 117(3), 505516. https://doi.org/10.1037/0735-7044.117.3.505

Adams, T. (2016, February 14). Is there too much stress on stress? The Guardian. https://www.theguardian.com/ society/2016/feb/14/workplace-stress-hans-selye

Akhtar, S., Justice, L.V., Knott, L., Kibowski, F., \& Conway, M.A. (2018). The 'common sense' memory belief system and its implications. The International Journal of Evidence \& Proof, 22(3), 289-304. https://doi. org/10.1177/1365712718784045

Andreano, J.M., \& Cahill, L. (2006). Glucocorticoid release and memory consolidation in men and women. Psychological Science, 17(6), 466-470. https://doi.org/10.1111/j.1467-9280.2006.01729.x

Ashcraft, M.H. (2002). Math anxiety: Personal, educational, and cognitive consequences. Current Directions in Psychological Science, 11(5), 181-185. https://doi.org/10.1111/1467-8721.00196

Aston-Jones, G., Rajkowski, J., \& Cohen, J. (2000). Locus coeruleus and regulation of behavioral flexibility and attention. Progress in Brain Research, 126, 165-182. https://doi.org/10.1016/S0079-6123(00)26013-5

Baer, R. A., Smith, G.T., \& Allen, K. B. (2004). Assessment of mindfulness by self-report: The Kentucky Inventory of Mindfulness Skills. Assessment, 11(3), 191-206. https://doi.org/10.1177/1073191104268029

Bailis, K.L., \& Mueller, J.H. (1981). Anxiety, feedback, and self-reference in face recognition. Motivation and Emotion, 5(1), 85-96. https://doi.org/10.1007/bf00993664

Bannerman, R.L., Milders, M., \& Sahraie, A. (2010). Attentional bias to brief threat-related faces revealed by saccadic eye movements. Emotion, 10(5), 733-738. https://doi.org/10.1037/a0019354

Barlow, D.H. (2002). Anxiety and its disorders: The nature and treatment of anxiety and panic (2nd ed.). Guildford Press.

Becker, D. (2013). One nation under stress: The trouble with stress as an idea. Oxford University Press. https://doi. org/10.1093/acprof:osobl/9780199742912.001.0001

Becker, V.E., Tucker, D.M., Delville, Y., Mohr, D.C. (2006). Stress facilitates consolidation of verbal memory for a film but does not affect retrieval. Behavioral Neuroscience, 120(3), 518-527. https://doi.org/10.1037/07357044.120 .3 .518

Beltzer, M.L., Nock, M.K., Peters, B.J., \& Jamieson, J.P. (2014). Rethinking butterflies: The affective, physiological, and performance effects of reappraising arousal during social evaluation. Emotion, 14(4), 761-768. https://doi. org/10.1037/a0036326

Benton, T.R., Ross, D.F., Bradshaw, E., Thomas, W.N., \& Bradshaw, G.S. (2006). Eyewitness memory is still not common sense: Comparing jurors, judges and law enforcement to eyewitness experts. Applied Cognitive Psychology, 20(1), 115-129. https://doi.org/10.1002/acp.1171

Bergomi, C., Ströhle, G., Michalak, J., Funke, F., \& Berking, M. (2013). Facing the dreaded: Does mindfulness facilitate coping with distressing experiences? A moderator analysis. Cognitive Behaviour Therapy, 42(1), 21-30. https://doi.org/10.1080/16506073.2012.713391

Blascovich, J. (2008). Challenge and threat. In A.J. Elliot (Ed.), Handbook of approach and avoidance motivation (pp. 431-445). Psychology Press.

Blascovich, J., \& Mendes, W.B. (2010). Social psychophysiology and embodiment. In S.T. Fiske, D.T. Gilbert, \& G. Lindzey (Eds.), The handbook of social psychology (5th ed, pp. 194-227). Wiley.

Bornstein, B.H., Hullman, G., \& Miller, M.K. (2013). Stress, trauma, and wellbeing in the legal system: Where do we go from here? In M.K. Miller \& B.H. Bornstein (Eds.) Stress, Trauma, and Wellbeing in the Legal System (pp. 293-309). Oxford Press. https://doi.org/10.1093/acprof:oso/9780199829996.001.0001 
Bornstein, B.H., O’Bryant, S., \& Zickafoose, D. (2008). Intuitions about arousal and eyewitness memory: Effects on mock jurors' judgments. Law \& Psychology Review, 32, 109-133.

Bornstein, B.H., \& Robicheaux, T.R. (2009). Methodological issues in the study of eyewitness memory and arousal. Creighton Law Review, 42, 525-547.

Brackmann, N., Otgaar, H., Sauerland, M., \& Jelicic, M. (2016). When children are the least vulnerable to false memories: A true report or a case of autosuggestion? Journal of Forensic Sciences, 61, S271-S275. https://doi. org/10.1111/1556-4029.12926

Bradley, M.M., \& Lang, P.J. (1999). Affective norms for English words (ANEW): Instruction manual and affective ratings. Technical Report C-1. The Center for Research in Psychophysiology, University of Florida.

Bray, N. (2015). Reappraising pain. Nature Reviews Neuroscience, 16(3), 124-125. https://doi.org/10.1038/nrn3919

Brewer, N., Vagadia, A.N., Hope, L., \& Gabbert, F. (2018). Interviewing witnesses: Eliciting coarse-grain information. Law and Human Behavior, 42(5), 458-471. https://doi.org/10.1037/lhb0000294

Brigham J.C., Maass A., Martinez, D., \& Wittenberger G. (1983). The effect of arousal on facial recognition. Basic and Applied Social Psychology, 4(3), 279-93. https://doi.org/10.1207/s15324834bas p0403_6

Brown, T.I., Uncapher, M.R., Chow, T.E., Eberhardt, J.L., \& Wagner, A.D. (2017). Cognitive control, attention, and the other race effect in memory. PLoS One, 12(3), e0173579. https://doi.org/10.1371/journal.pone.0173579

Buchanan, T.W., \& Lovallo, W.R. (2001). Enhanced memory for emotional material following stress-level cortisol treatments in humans. Psychoneuroendocrinology, 26(3), 307-317. https://doi.org/10.1016/S03064530(00)00058-5

Buchanan, T.W., \& Tranel, D. (2008). Stress and emotional memory retrieval: Effects of sex and cortisol response. Neurobiology of Learning and Memory, 89(2), 134-141. https://doi.org/10.1016/j.nlm.2007.07.003

Buchanan, T.W., Tranel, D., \& Adolphs, R. (2006). Impaired memory retrieval correlates with individual differences in cortisol response but not autonomic response. Learning \& Memory, 13(3), 382-387. https://doi.org/10.1101/ $\operatorname{lm} .206306$

Buckhout, R., Alper, A., Chern, S., Silverberg, G., \& Slomovits, M. (1974). Determinants of eyewitness performance on a lineup. Bulletin of the Psychonomic Society, 4(3), 191-192. https://doi.org/10.3758/bf03334241

Burke, A., Heuer, F., \& Reisberg, D. (1992). Remembering emotional events. Memory \& Cognition, 20(3), 277-290. https://doi.org/10.3758/BF03199665

Burton, A.M. (2013). Why has research in face recognition progressed so slowly? The importance of variability. Quarterly Journal of Experimental Psychology, 66(8), 1467-1485. https://doi.org/10.1080/17470218.2013.80 0125

Cahill, L. (2012). A half-truth is a whole lie: On the necessity of investigating sex influences on the brain. Endocrinology, 153(6), 2541-2543. https://doi.org/10.1210/en.2011-2167

Cahill, L., Gorski, L., \& Le, K. (2003). Enhanced human memory consolidation with post-learning stress: Interaction with the degree of arousal at encoding. Learning \& Memory, 10(4), 270-274. https://doi.org/10.1101/lm.62403

Cannon, W.B. (1929). Bodily changes in pain, hunger, fear, and rage: An account of recent researches into the function of emotional excitement (2nd ed.). Appleton.

Christianson, S-A. (1992). Emotional stress and eyewitness memory: A critical review. Psychological Bulletin, 112(2), 284-309. https://doi.org/10.1037/0033-2909.112.2.284

Christianson, S-A. \& Loftus, E.F. (1987). Memory for traumatic events. Applied Cognitive Psychology, 1(4), $225-239$. https://doi.org/10.1002/acp.2350010402

Christianson, S-A, Loftus, E.F., Hoffman, H., \& Loftus, G.R. (1991). Eye fixations and memory for emotional events. Journal of Experimental Psychology: Leaning, Memory, and Cognition, 17(4), 693-701. https://doi. org/10.1037/0278-7393.17.4.693 
Clark, C.R., Geffen, G.M., \& Clark, L.B. (1987). Catecholamines and attention: II. Pharmacological studies in normal humans. Neuroscience and Biobehavioral Reviews, 11(4), 353-364. https://doi.org/10.1016/S01497634(87)80007-6

Clifford, B.R., \& Hollin, C.R. (1981). Effects of the type of incident and the number of perpetrators on eyewitness memory. Journal of Applied Psychology, 66(3), 364-370. https://doi.org/10.1037/0021-9010.66.3.364

Conway, M.A., Justice, L.V., \& Morrison, C.M. (2014). Beliefs about autobiographical memory. The Psychologist, 27, 502-505.

Crum, A.J., Akinola, M., Martin, A., \& Fath, S. (2017). The role of stress mindset in shaping cognitive, emotional, and physiological responses to challenging and threatening stress. Anxiety, Stress, \& Coping, 30(4), 1-17. https://doi. org/10.1080/10615806.2016.1275585

Crum, A.J., Salovey, P., \& Achor, S. (2013). Rethinking stress: The role of mindsets in determining the stress response. Journal of Personality and Social Psychology, 104(4), 716-733. https://doi.org/10.1037/a0031201

Cutler, B.L., Penrod, S.D., \& Martens, T.K. (1987). The reliability of eyewitness identification: The role of system and estimator variables. Law and Human Behavior, 11(3), 233-258. https://doi.org/10.2307/1393634

Daniels, K., \& Abma, J.C. (2020). Current contraceptive use among women aged 15-49: United States, 2017-2019. NCHS Data Brief prepared for the U.S. Department of Health and Human Services, Centers for Disease Control and Prevention. No. 388. https://www.cdc.gov/nchs/data/databriefs/db388-H.pdf

Daubenmier, J., Hayden, D., Chang, V., \& Epel, E. (2014). It's not what you think, it’s how you relate to it: Dispositional mindfulness moderates the relationship between psychological distress and the cortisol awakening response. Psychoneuroendocrinology, 48, 11-18. https://doi.org/10.1016/j.psyneuen.2014.05.012

Davis, J.A. (2016, June 22). Critical incident stress reactions from violent crime. Psychology Today. Retrieved from https://www.psychologytoday.com/us/blog/crimes-and-misdemeanors/201606/critical-incident-stressreactions-violent-crime

Davis, S.D., Peterson, D.J., Wissman, K.T., \& Slater, W.A. (2019). Physiological stress and face recognition: Differential effects of stress on accuracy and the confidence-accuracy relationship. Journal of Applied Research in Memory and Cognition, 8, 367-375. https://doi.org/10.1016/j.jarmac.2019.05.006

de Kloet, E.R., Joëls, M., \& Holsboer, F. (2005). Stress and the brain: From adaptation to disease. Nature Reviews Neuroscience, 6(6), 463-475. https://doi.org/10.1038/nrn1683

de Kloet, E.R., Oitzl, M.S., \& Joëls, M. (1999). Stress and cognition: Are corticosteroids good or bad guys? Trends in Neurosciences, 22(10), 422-426. https://doi.org/10.1016/S0166-2236(99)01438-1

de Quervain, D.J.-F., Henke, K., Aerni, A., Treyer, V., McGaugh, J.L., Berthold, T., Nitsch, R.M., Buck, A., Roozendaal, B., \& Hock, C. (2003). Glucocorticoid-induced impairment of declarative memory retrieval is associated with reduced block flow in the medial temporal lobe. European Journal of Neuroscience, 17(6), 1296-1302. https:// doi.org/10.1046/j.1460-9568.2003.02542.x

de Quervain, D.J.-F., Roozendaal, B., \& McGaugh, J.L. (1998). Stress and glucocorticoids impair retrieval of longterm spatial memory. Nature, 394(6695), 787-790. https://doi.org/10.1038/29542

de Quervain, D.J.-F., Roozendaal, B., Nitsch, R.M., McGaugh, J.L., \& Hock, C. (2000). Acute cortisone administration impairs retrieval of long-term declarative memory in humans. Nature Neuroscience, 3(4), 313-314. https://doi. org/10.1038/73873

Deffenbacher, K.A. (1994). Effects of arousal on everyday memory. Human Performance, 7(2), 141-161. https://doi. org/10.1207/s15327043hup0702_3

Deffenbacher, K.A., Bornstein, B.H., Penrod, S.D., \& McGorty, E.K. (2004). A meta-analytic review of the effects of high stress on eyewitness memory. Law and Human Behavior, 28(6), 687-706. https://doi.org/10.1007/ s10979-004-0565-x 
Deffenbacher, K.A. \& Loftus, E.F. (1982). Do jurors share a common understanding concerning eyewitness behavior? Law and Human Behavior, 6(1), 15-30. https://doi.org/10.1007/BF01049310

Dellapaolera, K.S. (2019). How does stress at time of identification affect eyewitness memory? (Doctoral Dissertation). Retrieved from ProQuest Dissertations and Theses Global database. (ProQuest No. 22582828)

Denson, T.F., Creswell, J.D., Terides, M., \& Blundell, K. (2014). Cognitive reappraisal increases neuroendocrine reactivity to acute social stress and physical pain. Psychoneuroendocrinology, 49(1), 69-78. https://doi. org/10.1016/j.psyneuen.2014.07.003

Denson, T.F., Spanovic, M., \& Miller, N. (2009). Cognitive appraisals and emotions predict cortisol and immune responses: A meta-analysis of acute laboratory social stressors and emotion inductions. Psychological Bulletin, 135(6), 823-853. https://doi.org/10.1037/a0016909

Diamond, D.M., Campbell, A.M., Park, C.R., Halonen, J., \& Zoladz, P.R. (2007). The temporal dynamics model of emotional processing: A synthesis on the neurobiological basis of stress-induced amnesia, flashbulb and traumatic memories, and the Yerkes-Dodson Law. Neural Plasticity, 2007, 60803. https://doi.org/10.1155/2007/60803

Diamond, R., \& Carey, S. (1986). Why faces are and are not special: an effect of expertise. Journal of Experimental Psychology: General, 115(2), 107-117. https://doi.org/10.1037/0096-3445.115.2.107

Dickerson, S.S., \& Kemeny, M.E. (2004). Acute stressors and cortisol responses: A theoretical integration and synthesis of laboratory research. Psychological Bulletin, 130(3), 355-391. https://doi.org/10.1037/0033-2909.130.3.355

Domes, G., Heinrichs, M., Reichwald, U., Hautzinger, M. (2002). Hypothalamic-pituitary-adrenal axis reactivity to psychological stress and memory in middle-aged women: High responders exhibit enhanced declarative memory performance. Psychoneuroendocrinology, 27(7), 843-853. https://doi.org/10.1016/s0306-4530(01)00085-3

Domes, G., Heinrichs, M., Rimmele, U., Reichwald, U., \& Hautzinger, M. (2004). Acute stress impairs recognition for positive words-Association with stress-induced cortisol secretion. Stress, 7(3), 173-181. https://doi.org/1 $0.1080 / 10253890412331273213$

Easterbrook, J.A. (1959). The effect of emotion on the utilization and the organization of behavior. Psychological Review, 66(3), 183-201. https://doi.org/10.1037/h0047707

Echterhoff, G., \& Wolf, O.T. (2012). The stressed eyewitness: The interaction of thematic arousal and post-event stress in memory for central and peripheral event information. Frontiers in Integrative Neuroscience, 6(57), 1-12. https://doi.org/10.3389/fnint.2012.00057

Everly, G.S., Jr., \& Lating, J.M. (2013). A clinical guide to the treatment of human stress response (3rd ed). Springer Science + Business Media.

Faul, F., Erdfelder, E., Lang, A.G., \& Buchner, A. (2007). G*Power 3: A flexible statistical power analysis program for the social, behavioral, and biomedical sciences. Behavior Research Methods, 39(2), 175-191. https://doi. org/10.3758/bf03193146

Fawcett, J.M., Fawcett, E., Peace, K.A., \& Christie, J. (2013). Of guns and geese: A meta-analytic review of the 'weapon focus' literature. Psychology, Crime, \& Law, 19(1), 35-66. https://doi.org/10.1080/10683166X.2011.599325

Fawcett, J.M., Peace, K.A., \& Greve, A. (2016). Looking down the barrel of a gun: What do we know about the weapon focus effect? Journal of Applied Research in Memory and Cognition, 5(3), 257-263. https://doi.org/10.1016/ jarmac.2016.07.005

Fiske, D.T. Gilbert, \& G. Lindzey (Eds.), The handbook of social psychology (5th ed, pp. 194-227). Wiley.

Gagnon, S.A., \& Wagner, A.D. (2016). Acute stress and episodic memory: Neurobiological mechanisms and behavioral consequences. Annals of The New York Academy of Sciences, 1369(1), 55-75. https://doi.org/10.1111/nyas. 12996

Goldfarb, E.V., Tompary, A., Davachi, L., \& Phelps, E.A. (2019). Acute stress throughout the memory cycle: Diverging effects on associative and item memory. Journal of Experimental Psychology: General, 148(1), 13-29. https://doi. org/10.1037/xge0000472 
Golding, J.M., Sanchez, R.P., \& Sego, S.A. (1996). Do you believe in repressed memories? Professional Psychology: Research and Practice, 27(5), 429-437. https://doi.org/10.1037/0735-7028.27.5.429

Groscup, J., \& Tallon, J. (2009). Theoretical models of jury decision-making. In J.D. Lieberman \& D.A. Krauss (Eds.), Psychology, crime and law series: Jury psychology: Social aspects of trial processes: Psychology in the courtroom, Vol 1. (p.41-65). Ashgate Publishing Co.

Hancock, P.J.B., Bruce, V., \& Burton, A.M. (2000). Recognition of unfamiliar faces. Trends in Cognitive Sciences, 4(9), 330-337. https://doi.org/10.1016/s1364-6613(00)01519-9

Hangen, E.J., Elliot, A.J., \& Jamieson, J.P. (2019). Stress reappraisal during a mathematics competition: Testing effects on cardiovascular approach-oriented states and exploring the moderating role of gender. Anxiety, Stress, and Coping, 32(1), 95-108. https://doi.org/10.1080/10615806.2018.1530049

Hayes, A.F. (2018). Introduction to mediation, moderation, and conditional process analysis (2nd Ed., ed.). The Guilford Press.

Hellhammer, J., \& Schubert, M. (2012). The physiological response to Trier Social Stress Test relates to subjective measures of stress during but not before or after the test. Psychoneuroendocrinology, 37(1), 119-124. https://doi. org/10.1016/jpsyneuen.2011.05.012

Henckens, M.J.A.G., Hermans, E.J., Pu, Z., Joëls, M., \& Fernández, G. (2009). Stressed memories: How acute stress affects memory formation in humans. The Journal of Neuroscience, 29(32), 10111-10119. https://doi. org/10.1523/JNEUROSCI.1184-09.2009

Henrich, J., Heine, S.J., \& Norenzayan, A. (2010). The weirdest people in the world? Behavioral and Brain Sciences, 33(2-3), 61-83. https://doi.org/10.1017/S0140525X0999152X

Henze, G.I., Zänkert, S., Urschler, D.F., Hiltl, T.J., Kudielka, B.M., Pruessner, J C., \& Wüst, S. (2017). Testing the ecological validity of the Trier Social Stress Test: Association with real-life exam stress. Psychoneuroendocrinology, 75, 52-55. https://doi.org/10.1016/j.psyneuen.2016.10.002

Herlitz, A., \& Lovén, J. (2013). Sex differences and the own-gender bias in face recognition: A meta-analytic review. Visual Cognition, 21 (9-10), 1306-1336. https://doi.org/10.1080/13506285.2013.823140

Hermans, E.J., Henckens, M.J., Joëls, M., \& Fernández, G. (2014). Dynamic adaptation of large-scale brain networks in response to acute stressors. Trends in Neurosciences, 37(6), 304-314. https://doi.org/10.1016/j. tins.2014.03.006

Hermans, E.J., van Marle, H.J., Ossewaarde, L., Henckens, M.J.A.G., Qin, S., van Kesteren, M.T.R., Schoots, V.C., Cousijn, H., Rijpkema, M., Oostenveld, R., \& Fernández, G. (2011). Stress-related noradrenergic activity prompts large-scale neural network reconfiguration. Science, 334(6059), 1151-1153. https://doi.org/10.1126/ science. 1209603

Het, S., Ramlow, G., \& Wolf, O.T. (2005). A meta-analytic review of the effects of acute cortisol administration on human memory. Psychoneuroendocrinology, 30(8), 771-784. https://doi.org/10.1016/j.psyneuen.2005.03.005

Heuer, F., \& Reisberg, D. (1990). Vivid memories of emotional events: The accuracy of remembered minutiae. Memory \& Cognition, 18(5), 496-505. https://doi.org/10.3758/bf03198482

Hidalgo, V., Pulopulos, M.M., Puig-Perez, S., Espin, L., Gomez-Amor, J., \& Salvador, A. (2015). Acute stress affects free recall and recognition of pictures differently depending on age and sex. Behavioral Brain Research, 292, 393-402. https://doi.org/10.1016/j.bbr.2015.07.011

Hidalgo, V., Pulopulos, M.M., \& Salvador, A. (2019). Acute psychosocial stress effects on memory performance: Relevance of age and sex. Neurobiology of Learning and Memory, 157, 48-60. https://doi.org/10.1016/j. nlm.2018.11.013

Holm, S. (1979). A simple sequentially rejective multiple test procedure. Scandinavian Journal of Statistics, 6(2), 65-70. https://doi.org/10.2307/4615733 
Hope, L. (2016). Evaluating the effects of stress and fatigue on police officer response and recall: A challenge for research, training, practice and policy. Journal of Applied Research in Memory and Cognition, 5(3), 239-245. https://doi.org/10.1016/j.jarmac.2016.07.008

Hope, L., Blocksidge, D., Gabbert, F., Sauer, J.D., Lewinski, W., Mirashi, A., \& Atuk, E. (2016). Memory and the operational witness: Police officer recall of firearms encounters as a function of active response role. Law \& Human Behavior, 40(1), 23-35. https://doi.org/10.1037/lhb0000159

Hosch, H.M., \& Bothwell, R.K. (1990). Arousal, description and identification accuracy of victims and bystanders. Journal of Social Behavior and Personality, 5(5), 481-488.

Hoscheidt, S.M., LaBar, K.S., Ryan, L., Jacobs, W.J., \& Nadel, L. (2014). Encoding negative events under stress: High subjective arousal is related to accurate emotional memory despite misinformation exposure. Neurobiology of Learning and Memory, 112, 237-247. https://doi.org/10.1016/j.nlm.2013.09.008

Hulse, L.M., \& Memon, A. (2006). Fatal impact? The effects of emotional arousal and weapon presence on police officers' memories for a simulated crime. Legal and Criminological Psychology, 11(2), 313-325. https://doi. org/10.1348/135532505X58062

Hupbach, A., \& Fieman, R. (2012). Moderate stress enhances immediate and delayed retrieval of educationally relevant material in healthy young men. Behavioral Neuroscience, 126(6), 819-825. https://doi.org/10.1037/a0030489

Hyman, I.E., Wulff, A.N., \& Thomas, A.K. (2018). Crime blindness: How selective attention and inattentional blindness can disrupt eyewitness awareness and memory. Behavioral Insights and the Brain Sciences, 5(2), 202208. https://doi.org/10.1177/2372732218786749

Jamieson, J.P., Crum, A.J., Goyer, J.P., Marotta, M.E., \& Akinola, M. (2018). Optimizing stress responses with reappraisal and mindset interventions: An integrated model. Anxiety, Stress, \& Coping, 31(3), 245-261. https:// doi.org/10.1080/10615806.2018.1442615

Jamieson, J.P., Mendes, W.B., Blackstock, E., \& Schmader, T. (2010). Turning the knots in your stomach into bows: Reappraising arousal improves performance on the GRE. Journal of Experimental Social Psychology, 46(1), 208212. https://doi.org/10.1016/j.jesp.2009.08.015

Jamieson, J.P., Mendes, W.B., \& Nock, M.K. (2013a). Improving acute stress responses: The power of reappraisal. Current Directions in Psychological Science, 22(1), 51-56. https://doi.org/10.1177/0963721412461500

Jamieson, J.P., Nock, M.K., \& Mendes, W.B. (2012). Mind over matter: Reappraising arousal improves cardiovascular and cognitive responses to stress. Journal of Experimental Psychology: General, 141(3), 417-422. https://doi. org/10.1037/a0025719

Jamieson, J.P., Nock, M.K., \& Mendes, W.B. (2013b). Changing the conceptualization of stress in social anxiety disorder: Affective and physiological consequences. Clinical Psychological Science, 1(4), 1-12. https://doi. org/10.1177/2167702613482119

Jamieson, J.P., Peters, B.J., Greenwood, E.J., \& Altose, A.J. (2016). Reappraising stress arousal improves performance and reduces evaluation anxiety in classroom exam settings. Social Psychology and Personality Science, 7(6), 579587. https://doi.org/10.1177/1948550616644656

Jarosz, A.F., \& Wiley, J. (2014). What are the odds? A practical guide to computing and reporting Bayes factors. The Journal of Problem Solving, 7(1), 2-9. https://doi.org/10.7771/1932-6246.1167

JASP Team. (2020). JASP (Version 0.13.1) [Computer software].

Jeffreys, H. (1961). Theory of probability (3rd ed.). Oxford University Press.

Jentsch, V.L. \& Wolf, O.T. (2020). The impact of emotion regulation on cardiovascular, neuroendocrine and psychological stress responses. Biological Psychology, 154, 107893. https://doi.org/10.1016/j.biopsycho.2020.107893

Joëls, M., \& Baram, T.Z. (2009). The neuro-symphony of stress. Nature Reviews Neuroscience, 10(6), 459-466. https:// doi.org/10.1038/nrn2632 
Joëls, M., Fernández, G., \& Roozendaal, B. (2011). Stress and emotional memory: A matter of timing. Trends in Cognitive Sciences, 15(6), 280-288. https://doi.org/10.1016/j.tics.2011.04.004

Joëls, M., Pu, Z., Wiegert, O., Oitzl, M.S., \& Krugers, H.J. (2006). Learning under stress: How does it work? Trends in Cognitive Sciences, 10(4), 152-158. https://doi.org/10.1016/j.tics.2006.02.002

Kanwisher, N., \& Yovel, G. (2006). The fusiform face area: a cortical region specialized for the perception of faces. Philosophical Transactions of the Royal Society B: Biological Sciences, 361(1476), 2109-2128. https://doi. org/10.1098/rstb.2006.1934

Karst, H., Berger, S., Erdmann, G., Schütz, G., \& Joëls, M. (2010). Metaplasticity of amygdalar responses to the stress hormone corticosterone. Proceedings of the National Academy of Sciences, 107(32), 14449-14454. https://doi. org/10.1073/pnas.0914381107

Kassin, S.M. (1984). Eyewitness identification: Victims versus bystanders. Journal of Applied Social Psychology, 14(6), 519-529. https://doi.org/10.1111/j.1559-1816.1984.tb02257.x

Kassin, S.M., \& Barndollar, K.A. (1992). The psychology of eyewitness testimony: A comparison of experts and prospective jurors. Journal of Applied Social Psychology, 22(16), 1241-1249. https://doi.org/10.1111/j.1559-1816.1992. tb00948.x

Kassin, S.M., Ellsworth, P.C., \& Smith, V.L. (1989). The 'general acceptance' of psychological research on eyewitness testimony: A survey of the experts. American Psychologist, 44(8), 1089-1098. https://doi.org/10.1037/0003066X.44.8.1089

Kassin, S.M., Tubb, V.A., Hosch, H.M., \& Memon, A. (2001). On the 'general acceptance' of eyewitness testimony research: A new survey of the experts. American Psychologist, 56(5), 405-416. https://doi.org/10.1037/0003066X.56.5.405

Kees, J., Berry, C., Burton, S., \& Sheehan, K. (2017). An analysis of data quality: Professional panels, student subject pools, and Amazon's Mechanical Turk. Journal of Advertising, 46(1), 141-155. https://doi.org/10.1080/0091 3367.2016.1269304

Kim, E.J., Pellman, B., \& Kim, J.J. (2015). Stress effects on the hippocampus: A critical review. Learning \& Memory, 22(9), 411-416. https://doi.org/10.1101/lm.037291.114

Kirschbaum, C., Kudielka, B.M., Gaab, J., Schommer, N.C., \& Hellhammer, D.H. (1999). Impact of gender, menstrual cycle phase, and oral contraceptives on the activity of the hypothalamus-pituitary-adrenal axis. Psychosomatic Medicine, 61(2), 154-62. https://doi.org/10.1097/00006842-199903000-00006

Kirschbaum, C., Pirke, K.-M., \& Hellhammer, D.H. (1993). The 'Trier Social Stress Test': A tool for investigating psychobiological stress responses in a laboratory setting. Neuropsychobiology, 28(1-2), 76-81. https://doi. org/10.1159/000119004

Koo, T.K., \& Li, M.Y. (2016). A guideline of selecting and reporting intraclass correlation coefficients for reliability research. Journal of Chiropractic Medicine, 15(2), 155-163. https://doi.org/10.1016/j.jcm.2016.02.012

Kramer, T.H., Buckhout, R., \& Eugenio, P. (1990). Weapon focus, arousal, and eyewitness memory: Attention must be paid. Law and Human Behavior, 14(2), 167-184. https://doi.org/10.2307/1393597

Krix, A.C., Sauerland, M., Raymaekers, L.H.C., Memon, A., Quaedflieg, C.W.E.M., \& Smeets, T. (2016). Eyewitness evidence obtained with the Self-Administered Interview is unaffected by stress. Applied Cognitive Psychology, 30(1), 103-12. https://doi.org/10.1002/acp.3173

Kudiekla, B.M., Hellhammer, D.H., \& Wust, S. (2009). Why do we respond so differently? Reviewing determinants of human salivary cortisol responses to challenge. Psychoneuroendocrinology, 34, 2-18. https://doi.org/10.1016/j. psyneuen.2008.10.004

Kuhlmann, S., Kirschbaum, C., \& Wolf, O.T. (2005a). Effects of oral cortisol treatment in healthy young women on memory retrieval of negative and neutral words. Neurobiology of Learning and Memory, 83(2), 158-162. https:// doi.org/10.1016/j.nlm.2004.09.001 
Kuhlmann, S., Piel, M., \& Wolf, O.T. (2005b). Impaired memory retrieval after psychosocial stress in healthy young men. The Journal of Neuroscience, 25(11), 2977-2982. https://doi.org/10.1523/jneurosci.5139-04-2005

Kuhlmann, S., \& Wolf, O.T. (2006). Arousal and cortisol interact in modulating memory consolidation in healthy young men. Behavioral Neuroscience, 120(1), 217-223. https://doi.org/10.1037/0735-7044.120.1.217

Lanciano, T., \& Curci, A. (2011). Memories for emotional events: The accuracy of central and peripheral details. Europe's Journal of Psychology, 7(2), 323-336. https://doi.org/10.5964/ejop.v7i2.132

Lazarus, R.S. (1991). Progress on a cognitive-motivational-relational theory of emotion. American Psychologist, 46(8), 819-834. https://doi.org/10.1037/0003-066X.46.8.819

Leitenberg, H. (1990). Handbook of social and evaluation anxiety. Plenum Press.

Li, S., Weerda, R., Guenzel, F., Wolf, O.T., \& Thiel, C.M. (2013). ADRA2B genotype modulates effects of acute psychosocial stress on emotional memory retrieval in healthy young men. Neurobiology of Learning and Memory, 103, 11-18. https://doi.org/10.1016/j.nlm.2013.03.006

Li, S., Weerda, R., Milde, C., Wolf, O.T., \& Thiel, C.M. (2014). Effects of acute psychosocial stress on neural activity to emotional and neutral faces in a face recognition paradigm. Brain Imaging and Behavior, 8(4), 598-610. https://doi.org/10.1007/s11682-013-9287-3

Liu, J.J.W., Ein, N., Gervasio, J., \& Vickers, K. (2019). The efficacy of stress reappraisal interventions on stress responsivity: A meta-analysis and systematic review of existing evidence. PLoS ONE, 14(2), e0212854. https:// doi.org/10.1371/journal.pone.0212854

Loftus, E.F., Loftus, G.R., \& Messo, J. (1987). Some facts about "weapon focus". Law and Human Behavior, 11(1), 55-62. https://doi.org/10.1007/BF01044839

Lovallo, W. (1975). The cold pressor test and autonomic function: A review and integration. Psychophysiology, 12(3), 268-282. https://doi.org/10.1111/j.1469-8986.1975.tb01289.x

Lupien, S.J., Maheu, F., Tu, M., Ficco, A., \& Schramek, T.E. (2007). The effects of stress and stress hormones on human cognition: Implications for the field of brain and cognition. Brain and Cognition, 65(3), 209-237. https://doi.org/10.1016/j.bandc.2007.02.007

Lupien, S.J., McEwen, B.S., Gunnar, M.R., \& Heim, C. (2009). Effects of stress throughout the lifespan on the brain, behaviour, and cognition. Nature Reviews Neuroscience, 10(6), 434-445. https://doi.org/10.1038/nrn2638

Maass, A., \& Kohnken, G. (1989). Eyewitness identification: Simulating the "weapon effect." Law and Human Behavior, 13(4), 397-408. https://doi.org/10.1007/bf01056411

Magnussen, S., Melinder, A., Stridbeck, U., \& Raja, A. (2010). Beliefs about factors affecting the reliability of eyewitness testimony: A comparison of judges, jurors and the general public. Applied Cognitive Psychology, 24(1), 122-133. https://doi.org/10.1002/acp.1550

Maheu, F.S., Collicutt, P., Kornik, R., Moszkowski, R., \& Lupien, S.J. (2005). The perfect time to be stressed: A differential modulation of human memory by stress applied in the morning or in the afternoon. Progress in NeuroPsychopharmacology \& Biological Psychiatry, 29(8), 1281-1288. https://doi.org/10.1016/j.pnpbp.2005.08.012

Maki, P.M., Mordecai, K.L., Rubin, L.H., Sundermann, E., Savarese, A., Eatough, E., \& Drogos, L. (2015). Menstrual cycle effects on cortisol responsivity and emotional retrieval following a psychosocial stressor. Hormones and Behavior, 74, 201-208. doi:10.1016/j.yhbeh.2015.06.023

Marr, C., Otgaar, H., Sauerland, M., Quaedflieg, C., \& Hope, L. (2020). The effects of stress on eyewitness memory: A survey of memory experts and laypeople. Memory \& Cognition, 49(3), 401-421. https://doi.org/10.3758/ s13421-020-01115-4

Massar, S.A.A., Pu, Z., Chen, C., \& Chee, M.W.L. (2020). Losses motivate cognitive effort more than gains in effort-based decision making and performance. Frontiers in Human Neuroscience, 14 (287), 1-9. https://doi. org/10.3389/fnhum.2020.00287 
Mather, M., \& Sutherland, M.R. (2011). Arousal-biased competition in perception and memory. Perspectives in Psychological Science, 6(2), 114-133. https://doi.org/10.1177/1745691611400234

McCabe, D.P., Roediger III, H.L., McDaniel, M.A., Balota, D.A., \& Hambrick, D.Z. (2010). The relationship between working memory capacity and executive functioning: Evidence for a common executive attention construct. Neuropsychology, 24(2), 222-243. https://doi.org/10.1037/a0017619

McCarthy, J., \& Goffin, R. (2004). Measuring job interview anxiety: Beyond weak knees and sweaty palms. Personnel Psychology, 57(3), 607-637. https://doi.org/10.1111/j.1744-6570.2004.00002.x

McCullough, A.M., Ritchey, M., Ranganath, C., \& Yonelinas, A. (2015). Differential effects of stress-induced cortisol responses on recollection and familiarity-based recognition memory. Neurobiology of Learning and Memory, 123, 1-10. https://doi.org/10.1016/j.nlm.2015.04.007

McGaugh, J.L. (2004). The amygdala modulates the consolidation of memories of emotionally arousing experiences. Annual Review of Neuroscience, 27, 1-28. https://doi.org/10.1146/annurev.neuro.27.070203.144.157

McGaugh, J.L. (2015). Consolidating memories. Annual Review of Psychology, 66, 1-24. https://doi.org/10.1146/ annurev-psych-010814-014954

McGugin, R. W., Newton, A. T., Gore, J. C., \& Gauthier, I. (2014). Robust expertise effects in right FFA. Neuropsychologia, 63, 135-144. https://doi.org/10.1016/j.neuropsychologia.2014.08.02

McManus, E., Talmi, D., Haroon, H., \& Muhlert, N. (2020). The effects of psychosocial stress on memory and cognitive ability: A meta-analysis. medRxiv. https://doi.org/10.1101/2020.11.30.20240705

Meissner, C.A., \& Brigham, J.C. (2001). Thirty years of investigating the own-race bias in face memory for faces: A meta-analytic review. Psychology, Public Policy, and Law, 7(1), 3-35. https://doi.org/1037/1076-8971.7.1.3

Memon, A., Havard, C., Clifford, B., Gabbert, F., \& Watt, M. (2011). A field evaluation of the VIPE system: A new technique for eliciting eyewitness identification evidence. Psychology, Crime, and Law, 17(8), 711-729. https:// doi.org/10.1080/10683160903524333

Mendes, W.B., Gray, H.M., Mendoza-Denton, R., Major, B., \& Epel, E.S. (2007). Why egalitarianism might be good for your health: Physiological thriving during stressful intergroup encounters. Psychological Science, 18(11), 991-8. https://doi.org/10.1111/j.1467-9280.2007.02014.x

Mendes, W.B., \& Park, J. (2014). Neurobiological concomitants of motivational states. In A.J. Elliot (Ed.), Advances in motivation science (pp. 233-270). Elsevier. https://doi.org/10.1016/bs.adms.2014.09.001

Metcalfe, J., Brezler, J.C., McNamara, J., Malette, G., \& Vuorre, M. (2019). Memory, stress, and the hippocampal hypothesis: Firefighters' recollections of the fireground. Hippocampus, 29(12), 1141-1149. https://doi. org/10.1002/hipo. 23128

Miller, R., Plessow, F., Kirschbaum, C., \& Stalder, T. (2013). Classification criteria for distinguishing cortisol responders from nonresponders to psychosocial stress: Evaluation of salivary cortisol pulse detection in panel designs. Psychosomatic Medicine, 75(9), 832-840. https://doi.org/10.1097/PSY.0000000000000002

Morgan, C.A., Hazlett, G., Doran, A., Garrett, S., Hoyt, G., Thomas, P., Baranoski, M., \& Southwick, S.M. (2004). Accuracy of eyewitness memory for persons encountered during exposure to highly intense stress. International Journal of Law and Psychiatry, 27(3), 265-279. https://doi.org/10.1016/j.ijlp.2004.03.004

Mueller, J.H., Bailis, K.L., \& Goldstein, A.G. (1979). Depth of processing and anxiety in facial recognition. British Journal of Psychology, 70(4), 511-515. https://doi.org/10.1111/j.2044-8295.1979.tb01724.x

Nelson, R.J., \& Kriegsfeld, L.J. (2016). An introduction to behavioral endocrinology (5th). Sinauer Associates, Inc. Publishers.

Nielsen, S.E., Segal, S.K., Worden, I.V., Yim, I.S., \& Cahill, L. (2013). Hormonal contraception use alters stress responses and emotional memory. Biological Psychology, 92(2), 257-266. https://doi.org/j.biopsycho.2012.10.007 
Noon, E., \& Hollin, C.R. (1987). Lay knowledge of eyewitness behaviour: A British survey. Applied Cognitive Psychology, 1(2), 143-153. https://doi.org/10.1002/acp.2350010207

Nowicki, S., Winograd, E., \& Millard, B.A. (1979). Memory for faces: A social learning analysis. Journal of Research in Personality, 13(4), 460-468. https://doi.org/10.1016/0092-6566(79)90008-4

Otgaar, H., Howe, M.L., Patihis, L., Merckelbach, H., Lynn, S.J., Lilienfeld, S.O., \& Loftus, E.F. (2019). The return of the repressed: The persistent and problematic claims of long-forgotten trauma. Perspectives on Psychological Science, 14(6), 1072-1095. https://doi.org/10.1177/1745691619862306

Paul, M., Lech, R.K., Scheil, J., Dierolf, A.M., Suchan, B., \& Wolf, O.T. (2016). Acute stress influences the discrimination of complex scenes and complex faces in young healthy men. Psychoneuroendocrinology, 66, 125-129. https://doi.org/10.1016/j.psyneuen.2016.01.007

Payne, J.D., Jackson, E.D., Ryan, L., Hoscheidt, S., Jacobs, J.W., Nadel, L. (2006). The impact of stress on neutral and emotional aspects of memory. Memory, 14(1), 1-6. https://doi.org/10.1080/09658210500139176

Peters, D. P. (1988). Eyewitness memory in a natural setting. In M.M. Gruneberg, P.E. Morris, \& R.N. Sykes (Eds.), Practical aspects of memory: Current research and issues (pp. 89-94). Wiley.

Peters, D.P. (1997). Stress, arousal, and children's eyewitness memory. In Stein, N.L., Ornstein, P.A., Tversky, B., \& Brainerd, C.J. (Eds). Memory for everyday and emotional events. Erlbaum.

Pezdek, K., Abed, E., \& Cormia, A. (2020). Elevated stress impairs the accuracy of eyewitness memory but not the confidence-accuracy relationship. Journal of Experimental Psychology: Applied. Advance online publication. https://doi.org/10.1037/xap000316

Pincus, D.B., \& Friedman, A.G. (2004). Improving children's coping with everyday stress: Transporting treatment interventions to the school settings. Clinical Child and Family Psychology Review, 7(4), 223-240. https://doi. org/10.1007/s10567-004-6087-8

Posthuma, R.A., Morgeson, F.P., \& Campion, M.A. (2002). Beyond employment interview validity: A comprehensive narrative review of recent research and trends over time. Personnel Psychology, 55(1), 1-81. https://doi. org/10.1111/j.1744-6570.2002.tb00103.x

Quaedflieg, C.W.E.M., Meyer, T., van Ruitenbeek, P., \& Smeets, T. (2017). Examining habituation and sensitization across repetitive laboratory stress inductions using the MAST. Psychoneuroendocrinology, 77, 175-181. https:// doi.org/10.1016/j.psyneuen.2016.12.009

Quaedflieg, C.W.E.M., \& Schwabe, L. (2018). Memory dynamics under stress. Memory, 26(3), 364-376. https://doi. org/10.1080/09658211.2017.1338299

Quaedflieg, C.W.E.M., Schwabe, L., Meyer, T. \& Smeets, T. (2013). Time dependent effects of stress prior to encoding on event-related potentials and 24 h delayed retrieval. Psychoneuroendocrinology, 38(12), 3057-3069. https:// doi.org/10.1016/j.psyneuen.2013.09.002

Quaedflieg, C.W.E.M., van de Ven, V., Meyer, T., Siep, N., Merckelbach, H., \& Smeets, T. (2015). Temporal dynamics of stress-induced alternations of intrinsic amygdala connectivity and neuroendocrine levels. PLoS One, 10(5), E0124141. https://doi.org/10.1371/journal.pone.0124141

Qualtrics XM. Copyright (C) 2020 Qualtrics. Qualtrics and all other Qualtrics product or service names are registered trademarks or trademarks of Qualtrics, Provo, UT, USA. http://qualtrics.com

Raftery, A.E. (1995). Bayesian model selection in social research. Sociological Methodology, 25, 111-163. https://doi. org/10.2307/271063

Rajcani, J., Solarikova, P., Turonova, D., \& Brezina, I. (2016). Heart rate variability in psychosocial stress: Comparison between laboratory and real-life setting. Activitas Nervosa Superior Rediviva, 58(3), 77-82.

Read, J.D., \& Desmarais, S.L. (2009). Lay knowledge of eyewitness issues: A Canadian evaluation. Applied Cognitive Psychology, 23(3), 301-326. https://doi.org/10.1002/acp.1459 
Robbins, T.W. (1984). Cortical noradrenaline, attention and arousal. Psychological Medicine, 14, 13-21. doi:10.1017/ s0033291700003032

Robicheaux, T.R. (2016). Stress and eyewitness memory: Timing of stressor and association with cortisol stress responding (Doctoral Dissertation). Retrieved from Dissertation Abstracts International. (Accession No. 2016-26518-083)

Robinson, A. (2018). Let's talk about stress: History of stress research. Review of General Psychology, 22(3), 334-342. https://doi.org/10.1037/gpr0000137

Roozendaal, B. (2002). Stress and memory: Opposing effects of glucocorticoids on memory consolidation and memory retrieval. Neurobiology of Learning and Memory, 78(3), 578-595. https://doi.org/10.1006/nlme.2002.4080

Roozendaal, B., McEwen, B.S., \& Chattarji, S. (2009). Stress, memory and the amygdala. Nature Reviews Neuroscience, 10(6), 423-433. https://doi.org/10.1038/nrn2651

Roozendaal, B., \& McGaugh, J.L. (2011). Memory modulation. Behavioral Neuroscience, 125(6), 797-824. https:// doi.org/10.1037/a0026187

Sammon, N., \& Bogue, J. (2015). The impact of attention on eyewitness identification and change blindness. Journal of European Psychology Students, 6(2), 95-103. https://doi.org/10.5334/jeps.db

Sarason, I.G. (1972). Experimental approaches to test anxiety: Attention and the uses of information. In C.D. Spielberger (ed.), Anxiety: Current Trends in Theory and Research, vol. 2. Academic Press.

Sato, W., \& Yoshikawa, S. (2013). Recognition memory for faces and scenes. The Journal of General Psychology, 140(1), 1-15. https://doi.org/10.1080/00221309.2012.710275

Sauer, J., \& Hope, L. (2016). The effects of divided attention at study and reporting procedure on regulation and monitoring for episodic recall. Acta Psychologica, 169, 143-156. https://doi.org/10.1016/j.actpsy.2016.05.015

Sauerland, M., Raymaekers, L.H.C., Otgaar, H., Memon, A., Waltjen, T.T., Nivo, M., Slegers, C., Broers, N., \& Smeets, T. (2016). Stress, stress-induced cortisol responses, and eyewitness identification performance. Behavioral Sciences and the Law, 34(4), 580-594. https://doi.org/10.1002/bsl.2249

Schmechel, R.S., O’Toole, T.P., Easterly, C., \& Loftus, E.F. (2006). Beyond the ken? Testing jurors' understanding of eyewitness reliability evidence. Jurimetrics, 46(2), 177-214.

Schönfeld, P., Ackermann, K., \& Schwabe, L. (2014). Remembering under stress: Different roles of autonomic arousal and glucocorticoids in memory retrieval. Psychoneuroendocrinology, 39(1), 249-256 https://doi.org/10.1016/j. psychneuen.2013.09.020

Schoofs, D., \& Wolf, O.T. (2009). Stress and memory retrieval in women: No strong impairing effect during the luteal phase. Behavioral Neuroscience, 123(3), 547-554. https://doi.org/10.1037/a0015625

Schoofs, D., Wolf, O.T., \& Smeets, T. (2009). Cold pressor stress impairs performance on working memory tasks requiring executive functions in healthy, young men. Behavioral Neuroscience, 123(5), 1066-1075. https://doi. org/10.1037/a0016980

Schwabe, L., Bohringer, A., Chatterjee, M., \& Schachinger, H. (2008a). Effects of pre-learning stress on memory for neutral, positive, and negative words: Differential roles of cortisol and autonomic arousal. Neurobiology of Learning and Memory, 90(1), 44-53. https://doi.org/10.1016/j.nlm.2008/02.002

Schwabe, L., Haddad, L. \& Schachinger, H. (2008b). HPA axis activation by a socially evaluated cold-pressor test. Psychoneuroendocrinology, 33(6), 890-895. https://doi.org/10.1016/j.psyneuen.2008.03.001

Schwabe, L., Joëls, M., Roozendaal, B., Wolf, O.T., \& Oitzl, M.S. (2012). Stress effects on memory: An update and integration. Neuroscience \& Biobehavioral Reviews, 36(7), 1740-1749. https://doi.org/10.1016/j. neubiorev.2011.07.002

Schwabe, L., \& Wolf, O.T. (2009). Stress prompts habit behavior in humans. Journal of Neuroscience, 29(22), 71917198. https://doi.org/jneurosci.0979-09.2009 
Schwabe, L., \& Wolf, O.T. (2010). Learning under stress impairs memory formation. Neurobiology of Learning and Memory, 93(2), 183-188. https://doi.org/10.1016/j.nlm.2009.09.009

Schwabe, L., \& Wolf, O.T. (2012). Stress modulates in engagement of multiple memory systems in classification learning. The Journal of Neuroscience, 32(32), 11042-11049. https://doi.org/10.1523/JNEUROSCI.1484-12.2012

Schwabe, L., \& Wolf, O.T. (2014). Timing matters: Temporal dynamics of stress effects on memory retrieval. Cognitive, Affective, \& Behavioral Neuroscience, 14(3), 1041-1048. https://doi.org/10.3758/s13415-014-0256-0

Seery, M.D. (2011). Resilience: A silver lining to experiencing adverse life events? Current Directions in Psychological Science, 20(6), 390-394. https://doi.org/10.1177/0963721411424740

Shermohammed, M., Davidow, J.Y., Somerville, L.H., \& Murty, V.P. (2019). Stress impacts the fidelity but not strength of emotional memories. Brain and Cognition, 133, 33-41. https://doi.org/10.1016/j.bandc.2018.09.001

Shields, G.S. (2020). Stress and cognition: A user's guide to designing and interpreting studies. Psychoneuroendocrinology, 112, 104475. https://doi.org/10.1016/j.psyneuen.2019.104475

Shields, G.S., Sazma, M.A., McCullough, A.M., \& Yonelinas, A.P. (2017). The effects of acute stress on episodic memory: A meta-analysis and integrative review. Psychological Bulletin, 143(6), 636-675. https://doi. org/10.1037/bul0000100

Shields, G.S., Sazma, M.A., \& Yonelinas, A.P. (2016). The effects of acute stress on core executive functions: A metaanalysis and comparison with cortisol. Neuroscience and Biobehavioral Reviews, 68, 651-668. https://doi. org/10.1016/j.neubiorev.2016.06.038

Shilton, A.L., Laycock, R., \& Crewther, S.G. (2017). The Maastricht Acute Stress Test (MAST): Physiological and subjective responses in anticipation, and post-stress. Frontiers in Psychology, 8(567), 1-10. https://doi. org/10.3389/fpsyg.2017.00567

Simons, D.J., \& Chabris, C.F. (2011). What people believe about how memory works: A representative survey of the U.S. population. PLoS ONE, 6(8), e22757. https://doi.org/10.1371/journal.pone.0022757

Smeets, T. (2011). Acute stress impairs memory retrieval independent of time of day. Psychoneuroendocrinology, 36(4), 495-501. https://doi.org/10.1016/j.psyneuen.2010.08.001

Smeets, T., Cornelisse, S., Quaedflieg, C.W.E.M., Meyer, T., Jelicic, M., \& Merckelbach, H. (2012). Introducing the Maastricht Acute Stress Test (MAST): A quick and non-invasive approach to elicit robust autonomic and glucocorticoid stress responses. Psychoneuroendocrinology, 37(12), 1998-2008. https://doi.org/10.1016/j. psyneuen.2012.04.012

Smeets, T., Giesbrecht, T., Jelicic, M., \& Merckelbach, H. (2007). Context-dependent enhancement of declarative memory performance following acute psychosocial stress. Biological Psychology, 76(1-2), 116-123. https://doi. org/10.1016/j.biopsycho.2007.07.001

Smeets, T., Otgaar, H., Candel, I., \& Wolf, O. T. (2008). True or false? Memory is differentially affected by stressinduced cortisol elevations and sympathetic activity at consolidation and retrieval. Psychoneuroendocrinology, 33(10), 1378-1386. https://doi.org/10.1016/j.psyneuen.2008.07.009

Smith, A.M., Dijkstra, K., Gordon, L.T., Romero, M., \& Thomas, A.K. (2019). An investigation into the impact of acute stress on encoding in older adults. Aging, Neuropsychology, and Cognition, 26(5), 749-766. https://doi.or g/10.1080/13825585.2018.1524438

Smith, A.M., Floerke, V.A., \& Thomas, A.K. (2016). Retrieval practice protects memory against acute stress. Science, 354(6315), 1046-1048. https://doi.org/10.1126/science.aah5067

Staal, M.A. (2004). Stress, cognition, and human performance: A literature review and conceptual framework. National Aeronautics \& Space Administration.

Stanny, C.J., \& Johnson, T.C. (2000). Effects of stress induced by a simulated shooting on recall by police and citizen witnesses. The American Journal of Psychology, 113(3), 359-386. https://doi.org/10.2307/1423364 
Strahler, J., Skoluda, N., Kappert, M.B., \& Nater, U.M. (2017). Simultaneous measurement of salivary cortisol and alpha-amylase: Application and recommendation. Neuroscience \& Biobehavioral Reviews, 83, 657-677. https:// doi.org/10.1016/j.neubiorev.2017.08.015

Sydeman, S.J., Cascardi, M., Poythress, N.G., \& Ritterbrand, L.M. (1997). Procedural justice in the context of civil commitment: A critique of Tyler's analysis. Psychology, Public Policy \& Law, 3(1), 207-221. https://doi. org/10.1037/1076-8971.3.1.207

Theunissen, T.P.M., Meyer, T., Memon, A., \& Weinsheimer, C.C. (2017). Adult eyewitness memory for single versus repeated traumatic events. Applied Cognitive Psychology, 31(2), 164-174. https://doi.org/10.1002/acp.3314

Thomas, A.K., \& Karanian, J.M. (2019). Acute stress, memory, and the brain. Brain and Cognition, 133, 1-4. https:// doi.org/10.1016/j.bandc.2019.04.004

Tinsley, Y. (2001). Even better than the real thing? The case for reform of identification procedures. International Journal of Evidence and Proof, 5(2), 99-110. https://doi.org/10.1177/136571270100500202

Tollenaar, M.S., Elzinga, B.M., Spinhoven, P., \& Everaerd, W. (2009). Autobiographical memory after acute stress in healthy young men. Memory, 17(3), 301-310. https://doi.org/10.1080/09658210802665845

Tooley, V., Brigham, J.C., Maas, A., \& Bothwell, R.K. (1987). Facial recognition: Weapon effect and attentional focus. Journal of Applied Social Psychology, 17(10), 845-859. https://doi.org/10.1111/j.1559-1816.1987.tb00294.x

Ulrich-Lai, Y.M., \& Herman, J.P. (2009). Neural regulation of endocrine and autonomic stress responses. Nature Reviews Neuroscience, 10(6), 397-409. https://doi.org/10.1038/nrn2647

Valentine, T., \& Mesout, J. (2008). Eyewitness identification under stress in the London Dungeon. Applied Cognitive Psychology, 23(2), 151-161. https://doi.org/10.1002/acp.1463

Vogel, S., \& Schwabe, L. (2016a). Stress in the zoo: Tracking the impact of stress on memory formation over time. Psychoneuroendocrinology, 71, 64-72. https://doi.org/10.1016/j.psyneuen.2016.04.027

Vogel, S. \& Schwabe, L. (2016b). Learning and memory under stress: Implications for the classroom. npj Science of Learning, 1, 16011. https://doi.org/10.1038/npjscilearn.2016.11

Watson, D., Clark, L.A., \& Tellegen, A. (1988). Development and validation of brief measures of positive and negative affect: The PANAS scales. Journal of Personality and Social Psychology, 54(6), 1063-1070. https://doi. org/10.1037/0022-3514.54.6.1063

Weissgerber, T.L., Milic, N.M., Winham, S.J., \& Garovic, V.D. (2015). Beyond bar and line graphs: Time for a new data presentation paradigm. PLoS Biology, 13(4), e1002128. https://doi.org/10.1371/journal.pbio.1002128

Wessel, I., van der Kooy, P., \& Merckelbach, H. (2000). Differential recall of central and peripheral details of emotional slides is not a stable phenomenon. Memory, 8(2), 95-109. https://doi.org/10.1080/096582100387641

Wiemers, U.S., Hamacher-Dang, T.C., Yonelinas, A.P., \& Wolf, O.T. (2019). Pre-encoding stress induced changes in perceived stress, blood pressure and cortisol are differentially associated with recollection and familiarity. Brain and Cognition, 133, 5-11. https://doi.org/10.1016/j.bandc.2018.03.013

Wiemers, U.S., Sauvage, M.M., Schoofs, D., Hamacher-Dang, T.C., \& Wolf, O.T. (2013). What we remember from a stressful episode. Psychoneuroendocrinology, 38(10), 2268-2277. https://doi.org/10.1016/j. psyneuen.2013.04.015

Wolf, O.T. (2008). The influence of stress hormones on emotional memory: Relevance for psychopathology. Acta Psychologica, 127(3), 513-531. https://doi.org/10.1016/j.actpsy.2007.08.002

Wolf, O.T. (2009). Stress and memory in humans: Twelve years of progress? Brain Research, 1293, 142-154. https:// doi.org/10.1016/j.brainres.2009.04.013

Wolf, O.T. (2012). Immediate recall influences the effects of pre-encoding stress on emotional episodic long-term memory consolidation in healthy young men. Stress, 15(3), 272-280. https://doi.org/10.3109/10253890.20 11.622012 
Wolf, O.T. (2017). Stress and memory retrieval: Mechanisms and consequences. Current Opinions in Behavioral Sciences, 14, 40-46. https://doi.org/10.1016/cobeha.2016.12.001

Woodhead, M.M., \& Baddeley, A.D. (1981). Individual differences and memory for faces, pictures, and words. Memory \& Cognition, 9(4), 368-370. https://doi.org/10.3758/bf03197561

Wulff, A.N., \& Thomas, A.K. (2021). The dynamic and fragile nature of eyewitness memory formation: Considering stress and attention. Frontiers in Psychology, 12:666724. https://doi.org/fpsyg.2021.666724

Yarmey, A.D., \& Jones, H.P.T. (1983). Is the psychology of eyewitness identification a matter of common sense? In S. Lloyd-Bostock \& B.R. Clifford (Eds.). Evaluating witness evidence (pp.13-40). Wiley.

Yerkes, R.M., \& Dodson, J.D. (1908). The relation of strength of stimulus to rapidity of habit formation. Journal of Comparative Neurology and Psychology, 18(5), 459-482. https://doi.org/10.1002/cne.920180503

Young, C.B., Raz, G., Everaerd, D., Beckmann, C.F., Tendolkar, I., Hendler, T., Fernández, G., \& Hermans, E.J. (2017). Dynamic shifts in large-scale brain network balance as a function of arousal. The Journal of Neuroscience, 37(2), 281-290. https://doi.org/10.1523/JNEUROSCI.1759-16.2016

Yuille, J.C., \& Cutshall, J.L. (1986). A case study of eyewitness memory of a crime. Journal of Applied Psychology, 71, 291-301. doi:10.1037/0021-9010.71.2.291

Zeidner, M. (1995). Coping with examination stress: Resources, strategies, outcomes. Anxiety, Stress \& Coping: An International Journal, 8(4), 279-298. https://doi.org/10.1080/10615809508249379

Zoladz, P.R., Clark, B., Warnecke, A., Smith, L., Tabar, J., \& Talbot, J.N. (2011). Pre-learning stress differentially affects long-term memory for emotional words, depending on temporal proximity to the learning experience. Physiology \& Behavior, 103(5), 467-476. https://doi.org/10.1016/j.physbeh.2011.01.016

Zoladz, P.R., Duffy, T.J., Mosley, B.E., Fiely, M.K., Nagle, H.E., Scharf, A.R., Brown, C.M., Earley, M.B., Rorabaugh, B.R., \& Dailey, A.M. (2018). Interactive influence of sex, stressor timing, and the BclI glucocorticoid receptor polymorphism on stress-induced alterations of long-term memory. Brain and Cognition, 133, 72-83. https:// doi.org/10.1016/j.band.c.2018.05.012

Zoladz, P.R., Kalchik, A.E., Hoffman, M.M., Aufdenkampe, R.L., Lyle, S.M., Peters, D.M., Brown, C.M., Cadle, C.E., Scharf, A.R., Dailey, A.M., Wolters, N.E., Talbot, J.N., \& Rorabaugh, B.R. (2014). ADRA2B deletion variant selectively predicts stress-induced enhancement of long-term memory in females. Psychoneuroendocrinology, 48, 111-122. https://doi.org/10.1016/j.psyneun.2014.06.012 


\section{Summary}

Understanding how acute stress affects eyewitness memory performance is critical for legal settings. Though this topic has been studied for decennia, several gaps in knowledge still remain. The overarching aim of this thesis was to combine the distinct theoretical and methodological strengths of the eyewitness and fundamental memory fields to enhance understanding of this topic. Across one exploratory survey and three laboratory experiments, I examined the effects of acute stress on both encoding and retrieval memory performance.

First, I asked current memory experts $(n=73)$ and laypeople $(n=109)$ to provide their level of agreement on several statements regarding the stress-memory relationship (Chapter 2). Results showed that a large proportion of both eyewitness and fundamental memory researchers agreed that retrieval stress impairs memory performance. However, most fundamental memory experts also agreed that encoding stress enhances memory, whereas most eyewitness experts disagreed with this statement. Additionally, laypeople differed from experts on some factors and endorsed some ideas that are not supported by empirical research, for example, that trained professionals such as police are less affected by stress and that stress causes repressed memories.

Next, I presented two experiments $(N=240)$ investigating acute encoding and retrieval stress on face recognition memory (Chapter 3). Across experiments, the stress manipulation successfully raised participants' subjective negative affect, blood pressure, and cortisol levels (Experiment 3). However, results from both experiments showed no statistically significant effects of acute stress on memory performance. Specifically, in Experiment 2, no statistically significant differences in face recognition performance between groups emerged either for encoding or retrieval stress. In Experiment 3, acute encoding stress neither affected face recognition nor an added assessment of word recognition.

Turning to retrieval stress, my final experiment $(N=177)$ examined the effects of retrieval stress on recognition and free recall performance and investigated an arousal reappraisal intervention to mitigate potential negative effects of stress on memory (Chapter 4). Though the stress manipulation was again confirmed, results showed no effects of retrieval stress on either type of memory performance and no effect of the intervention on memory performance, affect, task demands, or coping resources. 
As discussed in the final chapter (Chapter 5), findings from this thesis highlight the lack of consensus between experts regarding the stress-memory relationship and raise questions about the generalizability and reliability of past findings. Additionally, the results emphasize the ongoing need for methodologically-sound research on this topic, ideally through experiments combining the distinct strengths of the eyewitness and fundamental memory fields. 


\section{Acknowledgments}

It is impossible to express in words my gratitude for everyone involved in my $\mathrm{PhD}$ journey over the past few years. I'll do my best. To begin, I want to thank all those involved in creating and contributing to the House of Legal Psychology - I feel fortunate to have learned from and worked beside such a diverse, intelligent, and fun group. I will forever be appreciative of this unique experience.

In particular, I would like to thank my remarkable team of supervisors: Melanie Sauerland, Henry Otgaar, Conny Quaedflieg, and Lorraine Hope. I could not have asked for a better team. Melanie, for your meticulous attention to detail and your sincere, unwavering support; Henry, for your unbelievably fast responses to my emails and your endlessly cheery attitude; Conny, for your continual hands-on assistance and your rigorous commitment to quality; and Lorraine, for your crucial focus on real world application and your ceaseless encouragement and inspiration. I am so grateful for and have learned so much from each of you.

To my friends, near and far: thank you. This endeavor would not have been the same without my friends and colleagues in Maastricht and Portsmouth, who added so much joy to my life and truly kept me sane during these years. To my other friends around the world, this experience confirmed to me that distance doesn't matter - thanks for being my chosen family.

To Luke, you've been there for me every single day throughout this journey - for the good and the bad, the ups and the downs, whether we were ten thousand miles apart or together 24/7 in lockdown. Thank you for your endless support and love, for knowing whether I needed pep talks, laughs, or hugs, and for being my rock during this experience otherwise full of uncertainty.

To my family, I wouldn't have made it here without you. Mom \& Dad: Thanks for raising me with a broad worldview and always modeling curiosity and humility. Thanks also for your incessant interest in my work and for always pushing me further. You both inspire me so much and I'll forever be in awe of your thirst for knowledge, empathy, and endless enthusiasm. To Dylan, Lucy, and Ginya - you all mean the world to me. I can't wait to see us all continue to grow together.

Finally, I dedicate this thesis to my two grandmothers, Virginia Worthington Marr and Doris Sturgis Wayman. Growing up with you two as my role models has made me who I am today. 


\section{Dissemination}

\section{Publications}

Marr, C., Sauerland, M., Otgaar, H., Quaedflieg, C.W.E.M., \& Hope, L. (2018). The effect of acute stress on memory: How it helps and how it hurts. In Mind, 9(38), 1. https://www.in-mind.org/article/the-effect-of-acute-stress-on-memory-how-ithelps-and-how-it-hurts

Marr, C., Otgaar, H., Sauerland, M., Quaedflieg, C.W.E.M., \& Hope, L. (2020). The effects of stress on eyewitness memory: A survey of memory experts and laypeople. Memory \& Cognition, 49(3), 401-421. https://doi.org/10.3758/s13421-020-01115-4

Marr, C., Sauerland, M., Otgaar, H., Quaedflieg, C.W.E.M., \& Hope, L. (2021). Mitigating the negative effects of retrieval stress on memory: An arousal reappraisal intervention. Memory, 29(3), 330-344. https://doi.org/10.1080/09658211.2021. 1893750

Marr, C., Quaedflieg, C.W.E.M., Otgaar, H., Hope, L., \& Sauerland, M. (2021). Facing stress: Examining the effect of acute stress during encoding and retrieval on face recognition. Acta Psychologica, 219(103376). https://doi.org/10.1016/j.actpsy.2021.103376

Marr, C., Sauerland, M., Otgaar, H., Quaedflieg, C.W.E.M., \& Hope, L. (2021). The effects of acute stress on eyewitness memory: An integrative review for eyewitness researchers. Memory, 29(8), 1091-1100. https://doi.org/10.1080/09658211.2021.19 55935

\section{Conference Presentations}

Marr, C., Sauerland, M., Otgaar, H., Quaedflieg, C.W.E.M., \& Hope, L. (2018, June). The effect of stress on face recognition during encoding and retrieval. Poster presentation at the annual conference of the European Association of Law (EAPL), Turku, Finland.

Marr, C., Sauerland, M., Otgaar, H., Quaedflieg, C.W.E.M., \& Hope, L. (2019, June). The effect of stress during encoding and retrieval on face recognition. Poster presentation at the Society for Applied Research on Memory and Cognition (SARMAC), Cape Cod, USA. 


\section{Curriculum Vitae}

Carey MacLane Marr was born on June 6, 1994 in Waterbury, Connecticut in the United States. In 2012, she completed her secondary education at Durham Academy before starting university at Williams College. At Williams, she majored in English and Psychology and spent three years working in a psychology research lab. Supervised by Prof. dr. Catherine B. Stroud, she completed a thesis on the effects of early childhood adversity and the serotonin system on stress generation in a sample of adolescent girls. After graduating cum laude in June of 2016 with honors in psychology, Carey moved to Sydney, where she worked in a forensic psychology lab at the University of Sydney, led by Dr. Helen Paterson and Dr. Celine van Golde. In September 2017, she began the Erasmus Mundus joint doctoral degree at the House of Legal Psychology. Her graduate research examining the effects of acute stress on eyewitness memory was supervised by Dr. Melanie Sauerland, Prof. dr. Henry Otgaar, and Dr. Conny Quaedflieg, and Prof dr. Lorraine Hope at the University of Portsmouth. Primarily based at Maastricht University, she also spent six months at the University of Portsmouth. 


\section{Valorisation Addendum}

\section{Relevance of the Research}

Witness testimony is critically important to the justice system, yet decades of research has unearthed the challenges of being primarily reliant on eyewitness memory. As reported by the Innocence Project, eyewitness misidentifications are the foremost cause of wrongful convictions, with 63\% of DNA exonerations in the United States involving eyewitness misidentifications (2021). Because eyewitnesses are often stressed when witnessing a crime, it is imperative to understand how such acute stress may impact their memory reports and later testimony. Understanding the impact of stress at encoding as an estimator variable would allow for better reliability judgments to be made regarding eyewitness memory reports. In addition, eyewitnesses may also experience stress during police interviews or court trials. Considering acute stress as a system variable is also vital, as stress experienced during retrieval could have significant implications for obtaining reliable information from eyewitnesses. The current thesis examined both of these relevant research questions by investigating the effects of acute stress at encoding and retrieval on memory in eyewitness settings.

\section{Target Group and Services}

The theoretical implications of this thesis are useful for other academic researchers, as this thesis critiques past work on stress and memory and offers recommendations for future research on this topic. The applied implications of these findings could be of interest to police and a range of legal practitioners, including lawyers, judges, and expert witnesses. These groups would benefit from understanding contemporary research on the stressmemory relationship and how it may apply to eyewitness memory. For example, results from my survey show that eyewitness and fundamental memory experts do not generally show high levels of consensus on statements about the stress-memory relationship. Expert witnesses testifying in court on the effects of acute stress on memory performance should a) be aware of research that exists across the wider research domain and b) present a comprehensive report to judges and juries, indicating the complexity of the topic and the multitude of factors that influence the relationship. Findings from this thesis cast doubt on the strength and generalizability of previous research findings, indicating that police and legal practitioners should use caution when applying previous findings in eyewitness settings. The research findings presented in this thesis highlight the need for further research using contemporary and robust methodology. Such work would continue to 
enhance our understanding of the stress-memory relationship, ideally offering police and legal practitioners more definitive conclusions and allowing for applications to the real world.

\section{Innovation of the Research}

Understanding the relationship between acute stress and memory in eyewitness settings has been a topic of interest for decennia. In this thesis, I first highlighted the critical limitations of past work and used these criticisms to form more robust and rigorous methodology. Specifically, this thesis uniquely combines strengths from the eyewitness and fundamental memory research fields to better investigate the stress-memory relationship. In addition, in this thesis, I examined both encoding and retrieval stress, a distinction often overlooked or ignored by past eyewitness memory research. Each experiment in this thesis represents an important and novel step in understanding the acute stress effects on memory: from understanding contemporary beliefs about this relationship, to specifically investigating these effects for face recognition memory, to testing an arousal reappraisal intervention in a new setting. Finally, I hope the explicit recommendations embedded in this thesis will allow for further innovation in the field to better understand the stressmemory relationship in eyewitness settings.

\section{Implementation and Dissemination}

The findings of this thesis have been shared in several ways. All experiments have already been published or have been submitted for publication in international peer-reviewed academic journals. In addition, I have written and submitted a review article with recommendations for future research aimed at memory researchers. To ensure a broad reach, all published articles are open-access and are thus accessible to anyone. I have also presented this research at international academic conferences in Finland and the United States. In hopes of communicating my research to a layperson audience, I have additionally published an article in In Mind, an online magazine that aims to share psychological science with the broader public. The current findings have also been presented informally, including online (e.g., on Twitter, ResearchGate, etc.) and at department and laboratory meetings at Maastricht University (Netherlands), the University of Portsmouth (U.K.), and the University of Sydney (Australia). Communicating academic research to practitioners and the general public is essential for real world impact and I hope to carry on communicating science and helping translate research to practice throughout my career. 


\section{List of Appendices}

Appendix A: Supplementary materials (Chapter 2; Experiment 1)

Appendix B: Supplementary materials (Chapter 3; Experiments 2 \& 3)

Appendix C: Supplementary materials (Chapter 4; Experiment 4) 


\section{Appendix A: Supplementary materials (Chapter 2; Experiment 1)}

Table A.A

Survey Statements With Full Distribution of Participant Endorsement

\begin{tabular}{|c|c|c|c|c|}
\hline \multirow[t]{2}{*}{ Shorthand } & & \multicolumn{3}{|c|}{ Percentage of participant endorsement } \\
\hline & & $\begin{array}{c}\text { Eyewitness } \\
\text { memory experts } \\
n=37\end{array}$ & $\begin{array}{c}\text { Fundamental } \\
\text { memory experts } \\
n=36\end{array}$ & $\begin{array}{c}\text { Laypeople } \\
n=109\end{array}$ \\
\hline \multirow{5}{*}{$\begin{array}{l}\text { 1. high stress } \\
\text { impairs }\end{array}$} & Strongly agree & 48.6 & 31.6 & 65.1 \\
\hline & Somewhat agree & 45.9 & 44.4 & 28.4 \\
\hline & Somewhat disagree & 2.7 & 13.9 & 2.8 \\
\hline & Strongly disagree & 0.0 & 2.8 & 0.9 \\
\hline & Don't know & 2.7 & 2.8 & 2.8 \\
\hline \multirow[t]{5}{*}{ 2. police interview } & Strongly agree & 45.9 & 25.0 & 38.5 \\
\hline & Somewhat agree & 35.1 & 50.0 & 43.1 \\
\hline & Somewhat disagree & 5.4 & 8.3 & 11.9 \\
\hline & Strongly disagree & 2.7 & 5.6 & 0.9 \\
\hline & Don’t know & 10.8 & 11.1 & 5.5 \\
\hline \multirow{5}{*}{$\begin{array}{l}\text { 3. } \begin{array}{l}\text { stress impairs } \\
\text { retrieval }\end{array} \\
\text { t }\end{array}$} & Strongly agree & 40.5 & 47.2 & 34.9 \\
\hline & Somewhat agree & 45.9 & 44.4 & 43.1 \\
\hline & Somewhat disagree & 0.0 & 5.6 & 11.0 \\
\hline & Strongly disagree & 2.7 & 2.8 & 1.8 \\
\hline & Don’t know & 10.8 & 0.0 & 9.2 \\
\hline \multirow{5}{*}{$\begin{array}{l}\text { 4. stress enhances } \\
\text { encoding }\end{array}$} & Strongly agree & 8.1 & 13.9 & 10.1 \\
\hline & Somewhat agree & 24.3 & 63.9 & 23.9 \\
\hline & Somewhat disagree & 37.8 & 19.4 & 35.8 \\
\hline & Strongly disagree & 24.3 & 0.0 & 17.4 \\
\hline & Don’t know & 5.4 & 2.8 & 12.8 \\
\hline \multirow{5}{*}{$\begin{array}{l}\text { 5. children less } \\
\text { affected }\end{array}$} & Strongly agree & 2.7 & 0.0 & 13.8 \\
\hline & Somewhat agree & 2.7 & 2.8 & 13.8 \\
\hline & Somewhat disagree & 35.1 & 30.6 & 35.8 \\
\hline & Strongly disagree & 37.8 & 25.0 & 21.1 \\
\hline & Don't know & 21.6 & 41.7 & 15.6 \\
\hline
\end{tabular}




\begin{tabular}{|c|c|c|c|c|}
\hline \multicolumn{2}{|l|}{ Shorthand } & \multicolumn{3}{|c|}{ Percentage of participant endorsement } \\
\hline \multirow[t]{5}{*}{ 6. detail type } & Strongly agree & 24.3 & 27.8 & 14.7 \\
\hline & Somewhat agree & 54.1 & 52.8 & 30.3 \\
\hline & Somewhat disagree & 8.1 & 8.3 & 29.4 \\
\hline & Strongly disagree & 2.7 & 8.3 & 6.4 \\
\hline & Don't know & 10.8 & 2.8 & 19.3 \\
\hline \multirow[t]{5}{*}{ 7. test type } & Strongly agree & 16.2 & 22.2 & 34.9 \\
\hline & Somewhat agree & 40.5 & 50.0 & 40.4 \\
\hline & Somewhat disagree & 16.2 & 8.3 & 9.2 \\
\hline & Strongly disagree & 2.7 & 0.0 & 0.9 \\
\hline & Don't know & 24.3 & 19.4 & 14.7 \\
\hline \multirow{5}{*}{$\begin{array}{l}\text { 8. faces affected } \\
\text { differently }\end{array}$} & Strongly agree & 10.8 & 0.0 & 20.2 \\
\hline & Somewhat agree & 27.0 & 19.4 & 44.0 \\
\hline & Somewhat disagree & 24.3 & 33.3 & 8.3 \\
\hline & Strongly disagree & 8.1 & 5.6 & 2.7 \\
\hline & Don’t know & 29.7 & 41.7 & 23.9 \\
\hline \multicolumn{2}{|c|}{ 9. professionals less Strongly agree } & 0.0 & 0.0 & 26.6 \\
\hline affected & Somewhat agree & 13.5 & 19.4 & 37.6 \\
\hline & Somewhat disagree & 51.4 & 38.9 & 21.1 \\
\hline & Strongly disagree & 35.1 & 33.3 & 8.3 \\
\hline & Don't know & 0.0 & 8.3 & 6.4 \\
\hline \multirow{5}{*}{$\begin{array}{l}\text { 10. victims more } \\
\text { affected }\end{array}$} & Strongly agree & 13.5 & 11.1 & 35.3 \\
\hline & Somewhat agree & 62.2 & 47.2 & 43.1 \\
\hline & Somewhat disagree & 13.5 & 16.7 & 10.1 \\
\hline & Strongly disagree & 5.4 & 2.8 & 2.8 \\
\hline & Don’t know & 5.4 & 22.2 & 8.3 \\
\hline \multirow[t]{5}{*}{ 11. repression } & Strongly agree & 5.4 & 2.8 & 33.9 \\
\hline & Somewhat agree & 10.8 & 11.1 & 51.4 \\
\hline & Somewhat disagree & 10.8 & 19.4 & 5.5 \\
\hline & Strongly disagree & 64.9 & 50.0 & 1.8 \\
\hline & Don't know & 8.1 & 16.7 & 7.3 \\
\hline
\end{tabular}




\begin{tabular}{|c|c|c|c|c|}
\hline \multicolumn{2}{|l|}{ Shorthand } & \multicolumn{3}{|c|}{ Percentage of participant endorsement } \\
\hline \multirow[t]{5}{*}{ 12. violent events } & Strongly agree & 10.8 & 0.0 & 18.3 \\
\hline & Somewhat agree & 29.7 & 13.9 & 25.7 \\
\hline & Somewhat disagree & 43.2 & 41.7 & 27.5 \\
\hline & Strongly disagree & 10.8 & 13.9 & 9.2 \\
\hline & Don't know & 5.4 & 30.6 & 19.3 \\
\hline \multirow{5}{*}{$\begin{array}{l}\text { 13. emotional } \\
\text { better } \\
\text { remembered }\end{array}$} & Strongly agree & 27.0 & 22.2 & 23.9 \\
\hline & Somewhat agree & 35.1 & 38.9 & 28.4 \\
\hline & Somewhat disagree & 16.2 & 22.2 & 28.4 \\
\hline & Strongly disagree & 2.7 & 0.0 & 0.9 \\
\hline & Don't know & 18.9 & 16.7 & 18.3 \\
\hline \multirow[t]{5}{*}{14.} & Strongly agree & 13.5 & 11.1 & 9.2 \\
\hline & Somewhat agree & 48.6 & 58.3 & 26.6 \\
\hline & Somewhat disagree & 13.5 & 8.3 & 34.9 \\
\hline & Strongly disagree & 5.4 & 8.3 & 15.6 \\
\hline & Don't know & 18.9 & 13.9 & 13.8 \\
\hline \multirow[t]{5}{*}{ 15. severe stress } & Strongly agree & 27.0 & 19.4 & 27.5 \\
\hline & Somewhat agree & 56.8 & 44.4 & 35.8 \\
\hline & Somewhat disagree & 5.4 & 13.9 & 20.2 \\
\hline & Strongly disagree & 10.8 & 8.3 & 3.7 \\
\hline & Don't know & 0.0 & 13.9 & 12.8 \\
\hline \multirow[t]{5}{*}{ 16. short crime } & Strongly agree & 0.0 & 0.0 & 9.2 \\
\hline & Somewhat agree & 2.7 & 0.0 & 17.4 \\
\hline & Somewhat disagree & 24.3 & 30.6 & 41.3 \\
\hline & Strongly disagree & 67.6 & 61.1 & 12.8 \\
\hline & Don’t know & 5.4 & 8.3 & 19.3 \\
\hline \multirow[t]{5}{*}{ 17. abstractness } & Strongly agree & 10.8 & 8.3 & 22.0 \\
\hline & Somewhat agree & 27.0 & 33.3 & 35.8 \\
\hline & Somewhat disagree & 29.7 & 38.9 & 20.2 \\
\hline & Strongly disagree & 5.4 & 139. & 4.6 \\
\hline & Don't know & 27.0 & 5.6 & 17.4 \\
\hline
\end{tabular}




\begin{tabular}{|c|c|c|c|c|}
\hline Shorthand & \multirow[b]{2}{*}{ Strongly agree } & \multicolumn{3}{|c|}{ Percentage of participant endorsement } \\
\hline \multirow{5}{*}{$\begin{array}{l}\text { immediate } \\
\text { retrieval } \\
\text { enhances }\end{array}$} & & 0.0 & 11.1 & 13.8 \\
\hline & Somewhat agree & 29.7 & 11.1 & 33.0 \\
\hline & Somewhat disagree & 27.0 & 22.2 & 25.7 \\
\hline & Strongly disagree & 10.8 & 19.4 & 5.5 \\
\hline & Don't know & 32.4 & 36.1 & 22.0 \\
\hline \multirow{5}{*}{$\begin{array}{l}\text { 19. retrieval } \\
\text { timing }\end{array}$} & Strongly agree & 27.0 & 5.6 & 19.3 \\
\hline & Somewhat agree & 35.1 & 25.0 & 38.5 \\
\hline & Somewhat disagree & 13.5 & 36.1 & 19.3 \\
\hline & Strongly disagree & 2.7 & 8.3 & 4.6 \\
\hline & Don't know & 21.6 & 25.0 & 18.3 \\
\hline \multirow[t]{5}{*}{20.} & Strongly agree & 2.7 & 0.0 & 11.9 \\
\hline & Somewhat agree & 8.1 & 19.4 & 33.9 \\
\hline & Somewhat disagree & 18.9 & 44.4 & 23.9 \\
\hline & Strongly disagree & 24.3 & 25.0 & 6.4 \\
\hline & Don't know & 45.9 & 11.1 & 23.9 \\
\hline \multirow{5}{*}{$\begin{array}{l}\text { 21. older adults } \\
\text { less affected }\end{array}$} & Strongly agree & 0.0 & 2.8 & 7.3 \\
\hline & Somewhat agree & 5.4 & 5.6 & 14.7 \\
\hline & Somewhat disagree & 27.0 & 33.3 & 31.2 \\
\hline & Strongly disagree & 16.2 & 13.9 & 27.5 \\
\hline & Don't know & 51.4 & 44.4 & 19.3 \\
\hline \multirow{5}{*}{$\begin{array}{l}\text { 22. } \\
\text { primarily } \\
\text { ANS activity* }\end{array}$} & Strongly agree & 2.7 & 2.8 & \\
\hline & Somewhat agree & 35.1 & 25.0 & \\
\hline & Somewhat disagree & 10.8 & 30.6 & \\
\hline & Strongly disagree & 2.7 & 27.8 & \\
\hline & Don't know & 48.6 & 13.9 & \\
\hline \multirow{5}{*}{$\begin{array}{l}\text { 23. ANS } \\
\text { facilitates* }\end{array}$} & Strongly agree & 10.8 & 25.0 & \\
\hline & Somewhat agree & 37.8 & 69.4 & \\
\hline & Somewhat disagree & 8.1 & 2.8 & \\
\hline & Strongly disagree & 5.4 & 0.0 & \\
\hline & Don't know & 37.8 & 2.8 & \\
\hline
\end{tabular}




\begin{tabular}{|c|c|c|c|}
\hline \multicolumn{2}{|l|}{ Shorthand } & \multicolumn{2}{|c|}{ Percentage of participant endorsement } \\
\hline \multirow{5}{*}{$\begin{array}{l}\text { 24. rapid cortisol } \\
\text { is beneficial }\end{array}$} & Strongly agree & 0.0 & 11.1 \\
\hline & Somewhat agree & 13.5 & 41.7 \\
\hline & Somewhat disagree & 2.7 & 8.3 \\
\hline & Strongly disagree & 0.0 & 2.8 \\
\hline & Don't know & 83.8 & 36.1 \\
\hline \multirow{5}{*}{$\begin{array}{l}\text { 25. slow cortisol is } \\
\text { detrimental* }\end{array}$} & Strongly agree & 2.7 & 5.6 \\
\hline & Somewhat agree & 10.8 & 22.2 \\
\hline & Somewhat disagree & 2.7 & 16.7 \\
\hline & Strongly disagree & 0.0 & 13.9 \\
\hline & Don’t know & 83.8 & 41.7 \\
\hline \multirow{5}{*}{$\begin{array}{l}\text { 26. noradrenergic } \\
\text { alone }^{*}\end{array}$} & Strongly agree & 0.0 & 19.4 \\
\hline & Somewhat agree & 21.6 & 22.2 \\
\hline & Somewhat disagree & 5.4 & 16.7 \\
\hline & Strongly disagree & 2.4 & 2.8 \\
\hline & Don't know & 73.0 & 38.9 \\
\hline \multirow{5}{*}{$\begin{array}{l}\text { 27. glucocorticoid } \\
\text { alone* }^{*}\end{array}$} & Strongly agree & 0.0 & 0.0 \\
\hline & Somewhat agree & 16.2 & 30.6 \\
\hline & Somewhat disagree & 10.8 & 25.0 \\
\hline & Strongly disagree & 0.0 & 11.1 \\
\hline & Don't know & 73.0 & 33.3 \\
\hline \multirow{5}{*}{$\begin{array}{l}\text { 28. } \\
\text { HPA \& ANS } \\
\text { activated }^{*}\end{array}$} & Strongly agree & 2.7 & 8.3 \\
\hline & Somewhat agree & 13.5 & 19.4 \\
\hline & Somewhat disagree & 8.1 & 25.0 \\
\hline & Strongly disagree & 0.0 & 11.1 \\
\hline & Don't know & 75.7 & 36.1 \\
\hline \multirow{5}{*}{$\begin{array}{l}\text { 29. } H P A \& A N S \\
\text { retrieval }^{*}\end{array}$} & Strongly agree & 5.4 & 13.9 \\
\hline & Somewhat agree & 8.1 & 30.6 \\
\hline & Somewhat disagree & 0.0 & 8.3 \\
\hline & Strongly disagree & 0.0 & 2.8 \\
\hline & Don't know & 86.5 & 44.4 \\
\hline
\end{tabular}

Note. ${ }^{*}$ = statement presented only to expert sample. 
Table A.B

Percentage of Expert Agreement About Court Reliability and Research Basis for Each Statement

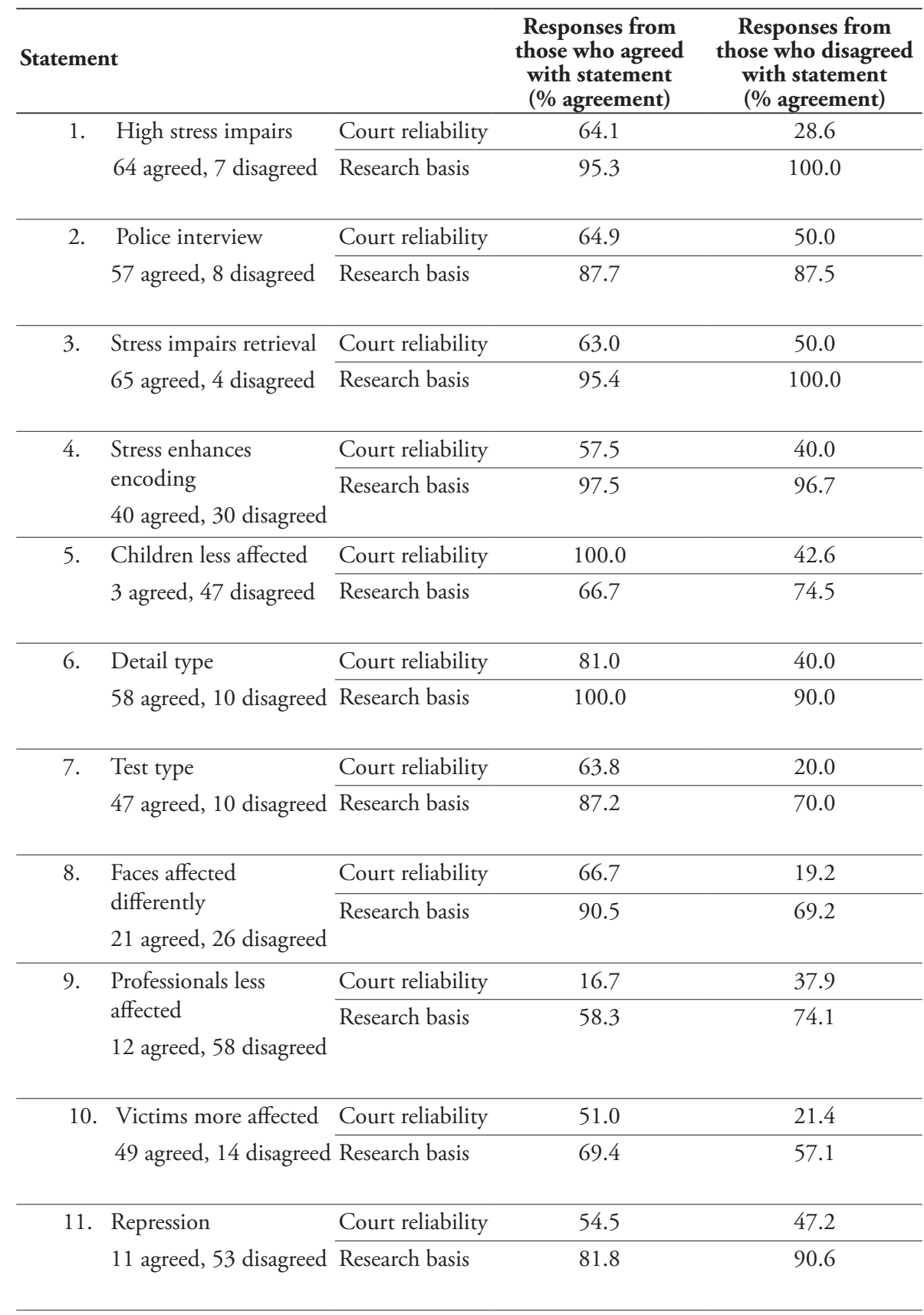




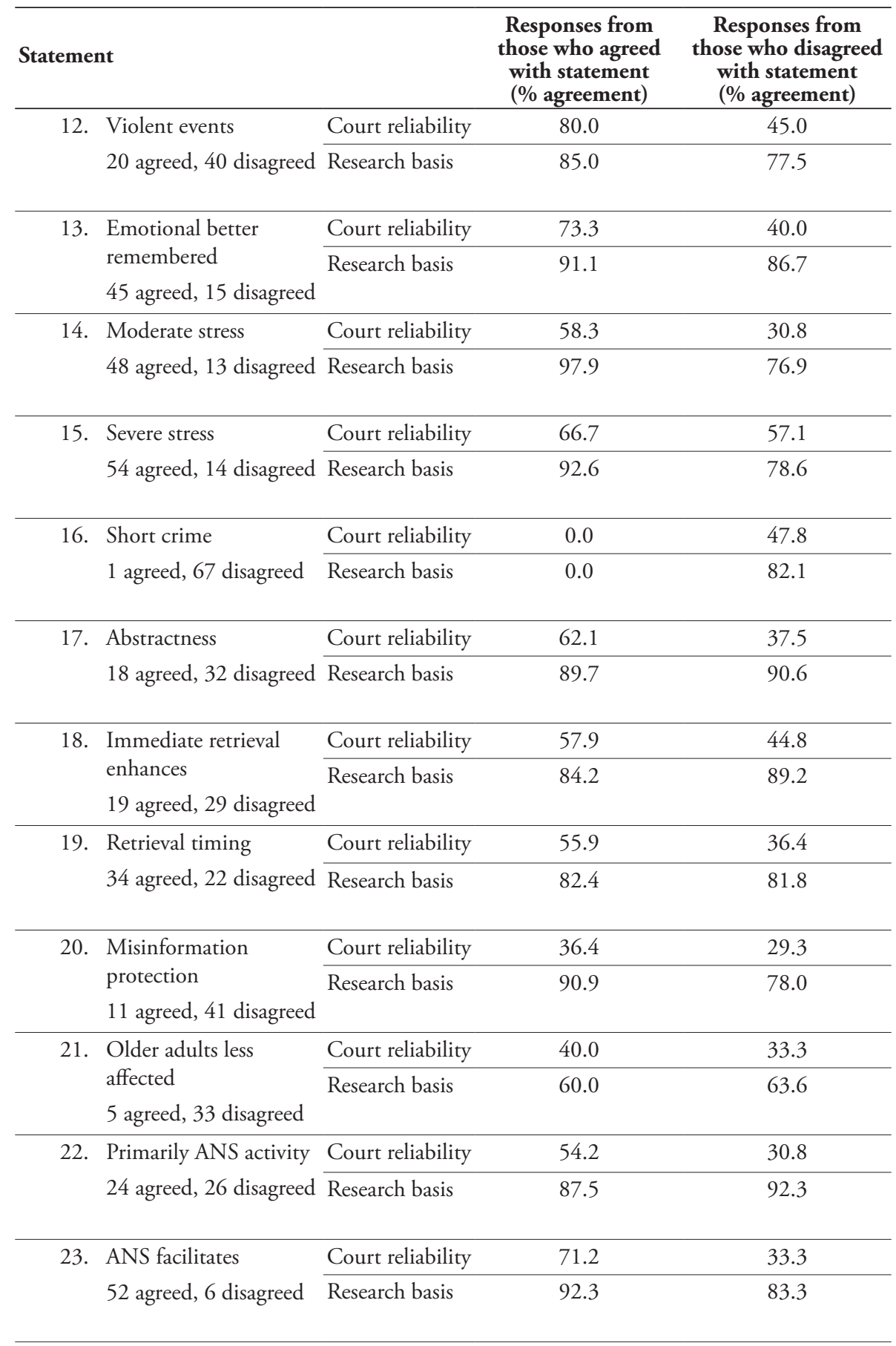




\begin{tabular}{|c|c|c|c|}
\hline Statement & & $\begin{array}{l}\text { Responses from } \\
\text { those who agreed } \\
\text { with statement } \\
\text { (\% agreement) }\end{array}$ & $\begin{array}{l}\text { Responses from } \\
\text { those who disagreed } \\
\text { with statement } \\
(\% \text { agreement })\end{array}$ \\
\hline \multirow{2}{*}{$\begin{array}{l}\text { Rapid cortisol is } \\
\text { beneficial }\end{array}$} & Court reliability & 50.0 & 20.0 \\
\hline & Research basis & 79.2 & 100.0 \\
\hline 24 agreed, 5 disagreed & & & \\
\hline \multirow{2}{*}{$\begin{array}{l}\text { Slow cortisol is } \\
\text { detrimental }\end{array}$} & Court reliability & 40.0 & 25.0 \\
\hline & Research basis & 86.7 & 100.0 \\
\hline 15 agreed, 12 disagreed & & & \\
\hline 26. Noradrenergic alone & Court reliability & 60.9 & 30.0 \\
\hline 23 agreed, 10 disagreed & Research basis & 95.7 & 100.0 \\
\hline 27. Glucocorticoid alone & Court reliability & 47.1 & 23.5 \\
\hline 17 agreed, 17 disagreed & Research basis & 94.1 & 88.2 \\
\hline 28. HPA \& ANS activated & Court reliability & 56.3 & 18.8 \\
\hline 16 agreed, 16 disagreed & Research basis & 87.5 & 93.8 \\
\hline 29. HPA \& ANS retrieval & Court reliability & 61.9 & 50.0 \\
\hline 21 agreed, 4 disagreed & Research basis & 85.7 & 100.0 \\
\hline
\end{tabular}

Note. Court reliability = percentage of this subset of participants who said the statement was reliable enough for psychologists to present in the courtroom. Research basis = percentage of this subset of participants who said their opinion was based on published, peer reviewed, and scientific research. 
Table A.C

Percentage of Memory Experts who Think Most People Believe This Statement to be True as a Matter of Common Sense

\begin{tabular}{clccc}
\hline Statement & Agree & Disagree & Don't know \\
\hline 1. & High stress impairs & 39.7 & 39.7 & 20.5 \\
\hline 2. & Police interview & 39.7 & 26.0 & 34.2 \\
\hline 3. & Stress impairs retrieval & 42.5 & 24.7 & 32.9 \\
\hline 4. & Stress enhances encoding & 30.1 & 42.5 & 27.4 \\
\hline 5. & Children less affected & 6.8 & 45.2 & 47.9 \\
\hline 6. & Detail type & 24.7 & 41.1 & 34.2 \\
\hline 7. & Test type & 15.1 & 37.0 & 47.9 \\
\hline 8. & Faces affected differently & 13.7 & 35.6 & 50.7 \\
\hline 9. & Professionals less affected & 47.9 & 23.3 & 28.8 \\
\hline 10. & Victims more affected & 38.4 & 21.9 & 39.7 \\
\hline 11. & Repression & 49.3 & 26.0 & 24.7 \\
\hline 12. & Violent events & 17.8 & 42.5 & 39.7 \\
\hline 13. & Emotional better remembered & 34.2 & 21.9 & 43.8 \\
\hline 14. & Moderate stress & 13.7 & 38.4 & 47.9 \\
\hline 15. & Severe stress & 28.8 & 31.5 & 39.7 \\
\hline 16. & Short crime & 9.6 & 43.8 & 46.6 \\
\hline 17. & Abstractness & 4.1 & 50.7 & 45.2 \\
\hline 18. & Immediate retrieval enhances & 5.5 & 41.1 & 53.4 \\
\hline 19. & Retrieval timing & 15.1 & 30.1 & 54.8 \\
\hline 20. & Misinformation protection & 4.1 & 45.2 & 50.7 \\
\hline 21. & Older adults less affected & 11.9 & 35.6 & 53.4 \\
\hline 22. & Primarily ANS activity & 2.7 & 33.6 & 61.6 \\
\hline 23. & ANS facilitates & 19.2 & 41.1 & 39.7 \\
\hline 24. & Rapid cortisol is beneficial & 2.7 & 45.2 & 52.1 \\
\hline 25. & Slow cortisol is detrimental & 1.4 & 45.2 & 53.4 \\
\hline 26. & Noradrenergic alone & 6.8 & 41.1 & 52.1 \\
\hline 27. & Glucocorticoid alone & 0.0 & 46.6 & 53.4 \\
\hline 28. & HPA \& ANS activated & 4.1 & 45.2 & 50.7 \\
\hline 29. & HPA \& ANS retrieval & 2.7 & 42.5 & 54.8 \\
\hline & & & \\
\hline & & & \\
\hline
\end{tabular}


Table A.D

Inferential Statistics for $2 \times 2 \times 2$ Tests Comparing Selections (Don't Know versus Agree/Disagree) in Eyewitness and Fundamental Memory Experts $(d f=1)$

\begin{tabular}{|c|c|c|c|c|}
\hline Statement & $\chi^{2}$ & $p$ & Adjusted $p$ & $\phi \phi \phi$ \\
\hline 1. High stress impairs* & & $>.999$ & .105 & .002 \\
\hline 2. Police interview* & & $>.999$ & .105 & .005 \\
\hline 3. Stress impairs retrieval* & & .115 & .105 & .237 \\
\hline 4. Stress enhances encoding* & & $>.999$ & .105 & .066 \\
\hline 5. Children less affected & 3.397 & .065 & .105 & .216 \\
\hline 6. Detail type* & & .358 & .105 & .159 \\
\hline 7. Test type & 0.254 & .614 & .105 & .059 \\
\hline 8. Faces affected differently & 1.134 & .287 & .105 & .125 \\
\hline 9. Professionals less affected* & & .115 & .105 & .210 \\
\hline 10. Victims more affected* & & .046 & .105 & .245 \\
\hline 11. Repression* & & .308 & .105 & .130 \\
\hline 12. Violent events & 7.885 & .005 & .105 & .329 \\
\hline 13. Emotional better remembered & 0.063 & .801 & .105 & .029 \\
\hline 14. Moderate stress & 0.336 & .562 & .105 & .068 \\
\hline 15. Severe stress* & & .025 & .105 & .275 \\
\hline 16. Short crime* & & .674 & .105 & .058 \\
\hline 17. Abstractness & 6.124 & .013 & .105 & .290 \\
\hline 18. Immediate retrieval enhances & 0.110 & .741 & .105 & .039 \\
\hline 19. Retrieval timing & 0.117 & .733 & .105 & .040 \\
\hline 20. Misinformation protection & 10.805 & .001 & .023 & .385 \\
\hline 21. Older adults less affected & 0.349 & .555 & .105 & .069 \\
\hline 22. Primarily ANS activity & 10.216 & .001 & .031 & .374 \\
\hline 23. ANS facilitates & 13.738 & $<.001$ & .005 & .434 \\
\hline 24. Rapid cortisol is beneficial & 17.319 & $<.001$ & $<.001$ & .487 \\
\hline 25. Slow cortisol is detrimental & 13.887 & $<.001$ & .005 & .436 \\
\hline 26. Noradrenergic alone & 7.254 & .007 & .105 & .315 \\
\hline 27. Glucocorticoid alone & 11.522 & .001 & .017 & .397 \\
\hline 28. HPA \& ANS activated & 11.601 & .001 & .017 & .399 \\
\hline 29. HPA \& ANS retrieval & 14.322 & $<.001$ & .004 & .443 \\
\hline
\end{tabular}

Note. $n=73$. Adjusted $p=$ Holm-Bonferroni adjustment for multiple comparisons. Bold $=$ adjusted $p$ significant at the .05 level. ${ }^{*}=$ Fisher's exact test instead of chi-square test (when expected cell sizes $<5)$. 


\section{Table A.E}

Inferential Statistics for $2 \times 2 \times 2$ Tests Comparing Selections (Don't Know versus Agree/Disagree) in Experts and Laypeople $(d f=1)$

\begin{tabular}{|c|c|c|c|c|}
\hline Statement & $\chi^{2}$ & $p$ & Adjusted $p$ & $\phi$ \\
\hline High stress impairs* & & & .220 & $<.001$ \\
\hline Police interview & 1.832 & .176 & .220 & .100 \\
\hline Stress impairs retrieval & 0.841 & .359 & .220 & .068 \\
\hline Stress enhances encoding & 3.939 & .047 & .220 & .147 \\
\hline 5. Children less affected & 6.454 & .011 & .220 & .188 \\
\hline 6. Detail type & 5.505 & .019 & .220 & .174 \\
\hline 7. Test type & 1.581 & .209 & .220 & .093 \\
\hline 8. $\quad$ Faces affected differently & 2.964 & .085 & .220 & .128 \\
\hline 9. $\quad$ Professionals less affected* & & .537 & .220 & .050 \\
\hline 10. Victims more affected & 1.385 & .239 & .220 & .087 \\
\hline 11. Repression & 1.285 & .257 & .220 & .084 \\
\hline 12. Violent events & 0.061 & .805 & .220 & .018 \\
\hline 13. Emotional better remembered & 0.009 & .926 & .220 & .007 \\
\hline 14. Moderate stress & 0.248 & .619 & .220 & .037 \\
\hline 15. Severe stress & 1.680 & .195 & .220 & .096 \\
\hline 16. Short crime & 5.505 & .019 & .220 & .174 \\
\hline 17. Abstractness & 0.030 & .861 & .220 & .013 \\
\hline 18. Immediate retrieval enhances & 2.655 & .103 & .220 & .121 \\
\hline 19. $\quad$ Retrieval timing & 0.658 & .417 & .220 & .060 \\
\hline 20. Misinformation protection & 0.551 & .458 & .220 & .055 \\
\hline 21. Older adults less affected & 16.881 & $<.001$ & $<.001$ & .305 \\
\hline
\end{tabular}




\section{Appendix B: Supplementary materials (Chapter 3; Experiments $2 \& 3$ )}

Table B.A

Inferential Statistics for Memory Performance Measures for No Stress, Low Cortisol Responders, and High Cortisol Responders in Experiment 3

\begin{tabular}{|c|c|c|c|c|c|c|c|c|}
\hline & & $\begin{array}{l}\text { Main } \\
\text { effect } \\
\text { Stress }\end{array}$ & $\begin{array}{c}\text { Main } \\
\text { effect } \\
\text { Stimulus }\end{array}$ & $\begin{array}{c}\text { Main } \\
\text { effect } \\
\text { Stress } \\
\text { stage }\end{array}$ & $\begin{array}{c}\text { Interaction } \\
\text { Stress x } \\
\text { Stimulus }\end{array}$ & $\begin{array}{c}\text { Interaction } \\
\text { Stress } \mathbf{x} \\
\text { Stress stage }\end{array}$ & $\begin{array}{l}\text { Interaction } \\
\text { Stimulus } \mathrm{x} \\
\text { Stress stage }\end{array}$ & $\begin{array}{c}\text { Interaction } \\
\text { Stress } \mathbf{x} \\
\text { Stimulus } \mathbf{x} \\
\text { Stress stage }\end{array}$ \\
\hline \multirow{3}{*}{$\begin{array}{l}\text { Overall } \\
\text { accuracy }\end{array}$} & $F$ & .372 & 2.793 & 2.337 & .229 & .900 & 15.204 & 1.347 \\
\hline & $p$ & .690 & .097 & .129 & .796 & .409 & $<.001$ & .264 \\
\hline & $\eta_{p}^{2}$ & .006 & .023 & .020 & .004 & .015 & .115 & .023 \\
\hline \multirow[t]{3}{*}{ Hits (\%) } & $F$ & .309 & 143.701 & .212 & .479 & 1.513 & 18.462 & .435 \\
\hline & $p$ & .735 & $<.001$ & .646 & .620 & .225 & $<.001$ & .648 \\
\hline & $\eta_{p}^{2}$ & .005 & .551 & .002 & .008 & .025 & .136 & .007 \\
\hline \multirow{3}{*}{$\begin{array}{l}\text { False } \\
\text { alarms } \\
(\%)\end{array}$} & $F$ & .268 & 141.312 & 2.393 & .032 & .787 & .039 & 2.460 \\
\hline & $p$ & .765 & $<.001$ & .125 & .968 & .458 & .845 & .090 \\
\hline & $\eta_{p}^{2}$ & .005 & .547 & .020 & .001 & .013 & $<.001$ & .040 \\
\hline \multirow[t]{3}{*}{$d^{\prime}$} & $F$ & .178 & 1.220 & 1.550 & .224 & 1.807 & 11.753 & .724 \\
\hline & $p$ & .837 & .272 & .216 & .800 & .169 & .001 & .487 \\
\hline & $\eta_{p}^{2}$ & .003 & .010 & .013 & .004 & .030 & .091 & .012 \\
\hline \multirow[t]{3}{*}{$c$} & $F$ & .435 & 245.392 & .795 & .410 & .513 & 7.836 & 1.695 \\
\hline & $p$ & .648 & $<.001$ & .374 & .664 & .600 & .006 & .188 \\
\hline & $\eta_{p}^{2}$ & .007 & .677 & .007 & .007 & .009 & .063 & .028 \\
\hline
\end{tabular}

Note. $N=120$ (no stress $n=59$, low responder $n=23$, high responder $n=38$ ). $d^{\prime}=$ sensitivity. $c=$ response bias. Findings that are statistically significant at the $p<.05$ level are in bold. 
Table B.B

Inferential Statistics for Memory Performance Measures, Excluding Outliers in Experiment 2

\begin{tabular}{llccc}
\hline & & $\begin{array}{c}\text { Main effect } \\
\text { stress stage }\end{array}$ & Main effect stress & $\begin{array}{c}\text { Interaction stress stage } \mathbf{x} \\
\text { stress }\end{array}$ \\
\hline Overall accuracy & $F$ & .042 & $\mathbf{2 . 7 1 6}$ & .298 \\
$N=118$ & $p$ & .837 & $\mathbf{. 0 4 8}$ & .827 \\
& $\eta_{p}{ }^{2}$ & $<.001$ & $\mathbf{. 0 6 7}$ & .008 \\
\hline Hits (\%) & $F$ & .734 & $\mathbf{2 . 8 8 5}$ & .981 \\
$N=115$ & $p$ & .394 & $\mathbf{. 0 3 9}$ & .405 \\
& $\eta_{p}{ }^{2}$ & .007 & $\mathbf{. 0 7 2}$ & .026 \\
\hline False alarms (\%) & $F$ & 2.556 & 1.150 & .710 \\
$N=112$ & $p$ & .113 & .332 & .548 \\
& $\eta_{p}{ }^{2}$ & .023 & .031 & .019 \\
& & & & .642 \\
d' & $F$ & 2.817 & 1.242 & .590 \\
$N=117$ & $p$ & .096 & .298 & .017 \\
& $\eta_{p}{ }^{2}$ & .024 & .032 & \\
\hline $\boldsymbol{c}$ & $F$ & $\mathbf{1 0 . 1 4 3}$ & .371 & .083 \\
$N=116$ & $p$ & $\mathbf{. 0 0 2}$ & .774 & .058 \\
\hline & $\eta_{p}{ }^{2}$ & $\mathbf{. 0 8 3}$ & .010 & \\
\hline
\end{tabular}

Note. $d^{\prime}=$ sensitivity. $c=$ response bias. Findings that are statistically significant at the $p<.05$ level are in bold. Outliers = scores that were $\geq 2.5 S D$ s from the mean. Post-hoc Bonferroni tests indicated no statistically significant difference for overall accuracy or hits between groups, all $p s \geq$ .064. Participants showed a more liberal response bias during the acute timepoint $(M=-.330, S E=$ $.033)$ than during the delayed timepoint $(M=-.207, S E=.036)$. 
Table B.C

Inferential Statistics for Memory Performance Measures, Excluding Guesses in Experiment 2

\begin{tabular}{lllll}
\hline & \multicolumn{2}{c}{$\begin{array}{l}\text { Main effect stress } \\
\text { stage }\end{array}$} & Main effect stress & $\begin{array}{l}\text { Interaction stress } \\
\text { stage x stress }\end{array}$ \\
\hline Overall accuracy & $F$ & .389 & 1.989 & .140 \\
& $p$ & .534 & .119 & .936 \\
& $\eta_{p}{ }^{2}$ & .003 & .049 & .004 \\
\hline Hits (\%) & $F$ & .094 & 1.351 & .177 \\
& $p$ & .760 & .261 & .912 \\
& $\eta_{p}{ }^{2}$ & .001 & .034 & .005 \\
\hline False alarms (\%) & $F$ & .433 & 1.153 & .126 \\
& $p$ & .512 & .331 & .944 \\
& $\eta_{p}{ }^{2}$ & .004 & .029 & .003 \\
\hline $\boldsymbol{d}$ & $F$ & .155 & 2.341 & .155 \\
& $p$ & .694 & .077 & .925 \\
& $\eta_{p}{ }^{2}$ & .001 & .057 & .004 \\
\hline $\boldsymbol{c}$ & $F$ & .175 & .348 & .189 \\
& $p$ & .676 & .791 & .904 \\
& $\eta_{p}{ }^{2}$ & .002 & .009 & .005 \\
\hline
\end{tabular}

Note. $N=120 . d^{\prime}=$ sensitivity. $c=$ response bias. Findings that are statistically significant at the $p<$ .05 level are in bold. Guesses = responses with ratings of less than $51 \%$ confidence. 


\section{Table B.D}

Bayes Factors for Memory Performance Measures in Experiment 2

\begin{tabular}{|c|c|c|c|c|c|c|}
\hline & $\begin{array}{l}\text { Main effect } \\
\text { stress stage }\end{array}$ & $\begin{array}{l}\text { Strength } \\
\text { (direction) }\end{array}$ & $\begin{array}{l}\text { Main effect } \\
\text { stress }\end{array}$ & $\begin{array}{l}\text { Strength } \\
\text { (direction) }\end{array}$ & $\begin{array}{l}\text { Interaction } \\
\text { stress stage } x \\
\text { stress }\end{array}$ & $\begin{array}{l}\text { Strength } \\
\text { (direction) }\end{array}$ \\
\hline & $\mathrm{BF}_{01}($ error $\%)$ & & $\mathrm{BF}_{01}($ error \%) & & $\mathrm{BF}_{01}($ error $\%)$ & \\
\hline $\begin{array}{l}\text { Overall } \\
\text { accuracy }\end{array}$ & $7.118(1.437)$ & $\begin{array}{l}\text { Substantial } \\
\text { (null) }\end{array}$ & $\mathbf{1 . 1 7 3}(1.236)$ & $\begin{array}{l}\text { Anecdotal } \\
\text { (null) }\end{array}$ & $\begin{array}{l}\mathbf{1 3 . 3 5 6} \\
(10.944)\end{array}$ & Strong (null) \\
\hline Hits (\%) & $5.106(1.051)$ & $\begin{array}{l}\text { Substantial } \\
\text { (null) }\end{array}$ & $3.346(0.415)$ & $\begin{array}{l}\text { Substantial } \\
\text { (null) }\end{array}$ & $7.985(7.152)$ & $\begin{array}{l}\text { Substantial } \\
\text { (null) }\end{array}$ \\
\hline $\begin{array}{l}\text { False } \\
\text { alarms } \\
(\%)\end{array}$ & $\mathbf{2 . 4 1 4}(0.009)$ & $\begin{array}{l}\text { Anecdotal } \\
\text { (null) }\end{array}$ & $\mathbf{3 . 6 0 4}(0.395)$ & $\begin{array}{l}\text { Substantial } \\
\text { (null) }\end{array}$ & $\begin{array}{l}9.139 \\
(42.532)\end{array}$ & $\begin{array}{l}\text { Substantial } \\
\text { (null) }\end{array}$ \\
\hline$d^{\prime}$ & $1.340(0.008)$ & $\begin{array}{l}\text { Anecdotal } \\
\text { (null) }\end{array}$ & $7.518(0.480)$ & $\begin{array}{l}\text { Substantial } \\
\text { (null) }\end{array}$ & $8.490(3.724)$ & $\begin{array}{l}\text { Substantial } \\
\text { (null) }\end{array}$ \\
\hline$c$ & $\mathbf{0 . 0 2 7}(2.853)$ & $\begin{array}{l}\text { Very strong } \\
\text { (alternative) }\end{array}$ & $11.326(0.553)$ & $\begin{array}{l}\text { Strong } \\
\text { (null) }\end{array}$ & $2.241(1.670)$ & $\begin{array}{l}\text { Anecdotal } \\
\text { (null) }\end{array}$ \\
\hline
\end{tabular}

Note. $d^{\prime}=$ sensitivity. $c=$ response bias. $\mathrm{BF}=$ Bayes factor. $\mathrm{BF}_{01}>100=$ decisive evidence for null, $\mathrm{BF}_{01} 30-100=$ very strong evidence for null, $\mathrm{BF}_{01} 10-30=$ strong evidence for null, $\mathrm{BF}_{01}$ 3-10 = substantial evidence for null, $\mathrm{BF}_{01} 1-3=$ anecdotal evidence for null, $\mathrm{BF}_{01}$ of $1=$ no evidence, $\mathrm{BF}_{01}$ $1 / 3-1=$ anecdotal evidence for alternative, $\mathrm{BF}_{01} 1 / 3-1 / 10=$ substantial evidence for alternative, $\mathrm{BF}_{01} 1 / 10-1 / 30=$ strong evidence for alternative, $\mathrm{BF}_{01} 1 / 30-1 / 100=$ very strong evidence for alternative, $\mathrm{BF}_{01}<1 / 100=$ decisive evidence for alternative (Jarosz \& Wiley, 2014; Jeffreys, 1961; Raftery, 1995). 
Table B.E

Inferential Statistics for Memory Performance Measures, Excluding Outliers in Experiment 3

\begin{tabular}{|c|c|c|c|c|c|c|c|c|}
\hline & & $\begin{array}{l}\text { Main } \\
\text { effect } \\
\text { Stress }\end{array}$ & $\begin{array}{c}\text { Main } \\
\text { effect } \\
\text { Stimulus }\end{array}$ & $\begin{array}{l}\text { Main } \\
\text { effect } \\
\text { Stress } \\
\text { stage }\end{array}$ & $\begin{array}{c}\text { Interaction } \\
\text { Stress } \mathbf{x} \\
\text { Stimulus }\end{array}$ & $\begin{array}{l}\text { Interaction } \\
\text { Stress } \mathbf{x} \\
\text { Stress stage }\end{array}$ & $\begin{array}{l}\text { Interaction } \\
\text { Stimulus } \mathbf{x} \\
\text { Stress stage }\end{array}$ & $\begin{array}{l}\text { Interaction } \\
\text { Stress } \mathbf{x} \\
\text { Stimulus } \mathbf{x} \\
\text { Stress stage }\end{array}$ \\
\hline \multirow{2}{*}{$\begin{array}{l}\text { Overall } \\
\text { accuracy }\end{array}$} & $F$ & 1.136 & 2.317 & .570 & .720 & .837 & 10.026 & 1.268 \\
\hline & $p$ & .254 & .131 & .452 & .398 & .362 & .002 & .262 \\
\hline$N=117$ & $\eta_{p}^{2}$ & .011 & .020 & .005 & .006 & .007 & .080 & .011 \\
\hline \multirow{3}{*}{$\begin{array}{l}\text { Hits (\%) } \\
N=116\end{array}$} & $F$ & .318 & 161.633 & .293 & .780 & 1.426 & 22.592 & .267 \\
\hline & $p$ & .574 & $<.001$ & .590 & .379 & .235 & $<.001$ & .606 \\
\hline & $\eta_{p}^{2}$ & .003 & .586 & .003 & .007 & .012 & .165 & .002 \\
\hline \multirow{3}{*}{$\begin{array}{l}\text { False } \\
\text { alarms } \\
(\%) \\
N=117 \\
\end{array}$} & $F$ & .576 & 172.561 & .868 & .154 & 1.651 & 1.180 & 1.918 \\
\hline & $p$ & .450 & $<.001$ & .353 & .696 & .201 & .280 & .169 \\
\hline & $\eta_{p}^{2}$ & .005 & .600 & .007 & .001 & .014 & .010 & .016 \\
\hline \multirow{3}{*}{$\begin{array}{l}\boldsymbol{d}^{\prime} \\
N=113\end{array}$} & $F$ & .270 & .455 & .475 & 1.434 & 3.435 & 8.170 & .493 \\
\hline & $p$ & .604 & .502 & .492 & .234 & .066 & .005 & .484 \\
\hline & $\eta_{p}^{2}$ & .002 & .004 & .004 & .013 & .030 & .069 & .004 \\
\hline \multirow{3}{*}{$\begin{array}{l}\boldsymbol{c} \\
N=116\end{array}$} & $F$ & .897 & 282.737 & 1.193 & .462 & .037 & 13.923, & .954 \\
\hline & $p$ & .346 & $<.001$ & .277 & .498 & .847 & $<.001$ & .331 \\
\hline & $\eta_{p}^{2}$ & .008 & .713 & .010 & .004 & $<.001$ & .109 & .008 \\
\hline
\end{tabular}

Note. $d^{\prime}=$ sensitivity. $c=$ response bias. Findings that are statistically significant at the $p<.05$ level are in bold. Outliers $=$ scores that were $\geq 2.5 S D$ s from the mean. 
Table B.F

Inferential Statistics for Memory Performance Measures, Excluding Guesses in Experiment 3

\begin{tabular}{|c|c|c|c|c|c|c|c|c|}
\hline & & $\begin{array}{l}\text { Main } \\
\text { effect } \\
\text { Stress }\end{array}$ & $\begin{array}{c}\text { Main } \\
\text { effect } \\
\text { Stimulus }\end{array}$ & $\begin{array}{l}\text { Main } \\
\text { effect } \\
\text { Stress } \\
\text { stage }\end{array}$ & $\begin{array}{c}\text { Interaction } \\
\text { Stress } \mathbf{x} \\
\text { Stimulus }\end{array}$ & $\begin{array}{c}\text { Interaction } \\
\text { Stress } \mathbf{x} \\
\text { Stress stage }\end{array}$ & $\begin{array}{l}\text { Interaction } \\
\text { Stimulus } \mathbf{x} \\
\text { Stress stage }\end{array}$ & $\begin{array}{c}\text { Interaction } \\
\text { Stress } \mathbf{x} \\
\text { Stimulus } \mathbf{x} \\
\text { Stress stage } \\
\end{array}$ \\
\hline \multirow{2}{*}{$\begin{array}{l}\text { Overall } \\
\text { accuracy }\end{array}$} & $F$ & 1.766 & 0.014 & .154 & 1.889 & 1.120 & 7.312 & .007 \\
\hline & $p$ & .186 & .907 & .696 & .172 & .292 & .008 & .933 \\
\hline$N=117$ & $\eta_{p}^{2}$ & .015 & $<.001$ & .001 & .016 & .009 & .050 & $<.001$ \\
\hline Hits (\%) & $F$ & 1.175 & 127.706 & 2.530 & 2.634 & .687 & 23.669 & .200 \\
\hline \multirow[t]{2}{*}{$N=116$} & $p$ & .281 & $<.001$ & .114 & .107 & .409 & $<.001$ & .656 \\
\hline & $\eta_{p}^{2}$ & .010 & .520 & .021 & .022 & .006 & .167 & .002 \\
\hline \multirow{3}{*}{$\begin{array}{l}\begin{array}{l}\text { False } \\
\text { alarms } \\
(\%)\end{array} \\
N=117\end{array}$} & $F$ & .002 & 102.769 & 9.956 & .007 & 2.346 & .072 & 1.035 \\
\hline & $p$ & .963 & $<.001$ & .002 & .932 & .128 & .789 & .331 \\
\hline & $\eta_{p}^{2}$ & $<.001$ & .466 & .078 & $<.001$ & .019 & $<.001$ & .009 \\
\hline$d^{\prime}$ & $F$ & .676 & .729 & 7.825 & 1.201 & 1.951 & 15.940 & .576 \\
\hline \multirow[t]{2}{*}{$N=113$} & $p$ & .413 & .395 & .006 & .275 & .165 & $<.001$ & .449 \\
\hline & $\eta_{p}^{2}$ & .006 & .006 & .062 & .010 & .016 & .119 & .005 \\
\hline$c$ & $F$ & .554 & 184.322 & 1.940 & .745 & .505 & 7.890 & .273 \\
\hline \multirow[t]{2}{*}{$N=116$} & $p$ & .458 & $<.001$ & .166 & .390 & .479 & .006 & .602 \\
\hline & $\eta_{p}^{2}$ & .005 & .610 & .016 & .006 & .004 & .063 & .002 \\
\hline
\end{tabular}

Note. $N=120 . d^{\prime}=$ sensitivity. $c=$ response bias. Findings that are statistically significant at the $p<$ .05 level are in bold. Guesses = responses with ratings of less than $51 \%$ confidence. 
Table B.G

Bayes Factors for Memory Performance Measures in Experiment 3

\begin{tabular}{|c|c|c|c|c|c|c|c|}
\hline & $\begin{array}{l}\text { Main } \\
\text { effect } \\
\text { Stress }\end{array}$ & $\begin{array}{l}\text { Main } \\
\text { effect } \\
\text { Stimulus }\end{array}$ & $\begin{array}{l}\text { Main } \\
\text { effect } \\
\text { Stress } \\
\text { stage }\end{array}$ & $\begin{array}{l}\text { Interaction } \\
\text { Stress } \mathbf{x} \\
\text { Stimulus }\end{array}$ & $\begin{array}{l}\text { Interaction } \\
\text { Stress } \mathbf{x} \\
\text { Stress stage }\end{array}$ & $\begin{array}{l}\text { Interaction } \\
\text { Stimulus } \mathbf{x} \\
\text { Stress stage }\end{array}$ & $\begin{array}{l}\text { Interaction } \\
\text { Stress } \mathbf{x} \\
\text { Stimulus } \mathbf{x} \\
\text { Stress stage } \\
\end{array}$ \\
\hline \multirow{2}{*}{$\begin{array}{l}\text { Overall } \\
\text { accuracy }\end{array}$} & 5.200 & 1.837 & 4.085 & 7.109 & 3.965 & 0.124 & 1.675 \\
\hline & $\begin{array}{l}\text { (1.012, } \\
\text { substantial, } \\
\text { null) }\end{array}$ & $\begin{array}{l}(4.159, \\
\text { anecdotal, } \\
\text { null) }\end{array}$ & $\begin{array}{l}(1.095, \\
\text { substantial, } \\
\text { null })\end{array}$ & $\begin{array}{l}(3.497, \\
\text { substantial, } \\
\text { null })\end{array}$ & $\begin{array}{l}\text { ( } 4.434, \\
\text { substantial), } \\
\text { null) }\end{array}$ & $\begin{array}{l}(4.163, \\
\text { substantial, } \\
\text { alternative) }\end{array}$ & $\begin{array}{l}(9.013, \\
\text { anecdotal, } \\
\text { null) }\end{array}$ \\
\hline \multirow[t]{2}{*}{ Hits (\%) } & 6.451 & $1.117 e-37$ & 9.524 & 4.602 & 3.419 & $9.635 e-4$ & 4.999 \\
\hline & $\begin{array}{l}(2.302, \\
\text { substantial, } \\
\text { null })\end{array}$ & $\begin{array}{l}(1.341, \\
\text { decisive, } \\
\text { alternative) }\end{array}$ & $\begin{array}{l}(2.550, \\
\text { substantial, } \\
\text { null })\end{array}$ & $\begin{array}{l}(4.505, \\
\text { substantial, } \\
\text { null) }\end{array}$ & $\begin{array}{l}(3.896, \\
\text { substantial, } \\
\text { null) }\end{array}$ & $\begin{array}{l}(4.521, \\
\text { decisive, } \\
\text { alternative) }\end{array}$ & $\begin{array}{l}\text { (6.561, } \\
\text { substantial, } \\
\text { null) }\end{array}$ \\
\hline \multirow{2}{*}{$\begin{array}{l}\text { False } \\
\text { alarms } \\
(\%)\end{array}$} & 7.790 & $2.029 e-36$ & 6.472 & 7.345 & 4.038 & 4.612 & 2.750 \\
\hline & $\begin{array}{l}(0.767, \\
\text { substantial, } \\
\text { null })\end{array}$ & $\begin{array}{l}(0.919, \\
\text { decisive, } \\
\text { alternative) }\end{array}$ & $\begin{array}{l}\text { ( } 3.041, \\
\text { substantial, } \\
\text { null) }\end{array}$ & $\begin{array}{l}(4.360, \\
\text { substantial, } \\
\text { null) }\end{array}$ & $\begin{array}{l}(5.402, \\
\text { substantial, } \\
\text { null) }\end{array}$ & $\begin{array}{l}(4.866, \\
\text { substantial, } \\
\text { null) }\end{array}$ & $\begin{array}{l}(6.445, \\
\text { anecdotal, } \\
\text { null })\end{array}$ \\
\hline \multirow[t]{2}{*}{$d^{\prime}$} & 6.607 & 3.859 & 7.565 & 6.045 & 1.408 & 0.133 & 3.083 \\
\hline & $\begin{array}{l}(0.013, \\
\text { substantial, } \\
\text { null })\end{array}$ & $\begin{array}{l}(0.008, \\
\text { substantial, } \\
\text { null })\end{array}$ & $\begin{array}{l}(0.010, \\
\text { substantial, } \\
\text { null })\end{array}$ & $\begin{array}{l}(3.667, \\
\text { substantial, } \\
\text { null) }\end{array}$ & $\begin{array}{l}(3.493, \\
\text { anecdotal, } \\
\text { null })\end{array}$ & $\begin{array}{l}(3.799, \\
\text { substantial, } \\
\text { alternative) }\end{array}$ & $\begin{array}{l}(6.358, \\
\text { substantial, } \\
\text { null) }\end{array}$ \\
\hline \multirow[t]{2}{*}{ 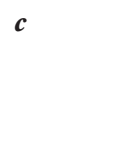 } & 6.712 & $2.639 e-58$ & 8.991 & 4.767 & 6.639 & 0.041 & 4.844 \\
\hline & $\begin{array}{l}(0.023, \\
\text { substantial, } \\
\text { null) }\end{array}$ & $\begin{array}{l}(2.829, \\
\text { decisive, } \\
\text { alternative) }\end{array}$ & $\begin{array}{l}(0.910, \\
\text { substantial, } \\
\text { null })\end{array}$ & $\begin{array}{l}(4.470, \\
\text { substantial, } \\
\text { null) }\end{array}$ & $\begin{array}{l}(7.860, \\
\text { substantial, } \\
\text { null) }\end{array}$ & $\begin{array}{l}(3.869, \\
\text { strong, } \\
\text { alternative) }\end{array}$ & $\begin{array}{l}(20.389, \\
\text { substantial, } \\
\text { null) }\end{array}$ \\
\hline
\end{tabular}

Note. $d^{\prime}=$ sensitivity. $c=$ response bias. Number in bold $=\mathrm{BF}_{01}$. Numbers in parentheses represent error percentage, strength, and direction. $\mathrm{BF}=$ Bayes factor. $\mathrm{BF}_{01}>100=$ decisive evidence for null, $\mathrm{BF}_{01} 30-100=$ very strong evidence for null, $\mathrm{BF}_{01} 10-30=$ strong evidence for null, $\mathrm{BF}_{01} 3-10=$ substantial evidence for null, $\mathrm{BF}_{01} 1-3=$ anecdotal evidence for null, $\mathrm{BF}_{01}$ of $1=$ no evidence, $\mathrm{BF}_{01}$ $1 / 3-1=$ anecdotal evidence for alternative, $\mathrm{BF}_{01} 1 / 3-1 / 10=$ substantial evidence for alternative, $\mathrm{BF}_{01} 1 / 10-1 / 30=$ strong evidence for alternative, $\mathrm{BF}_{01} 1 / 30-1 / 100=$ very strong evidence for alternative, $\mathrm{BF}_{01}<1 / 100$ = decisive evidence for alternative (Jarosz \& Wiley, 2014; Jeffreys, 1961; Raftery, 1995). 
Appendix C: Supplementary materials (Chapter 4; Experiment 4)

1. Stimuli: Bus, Living room, Kitchen, Traffic
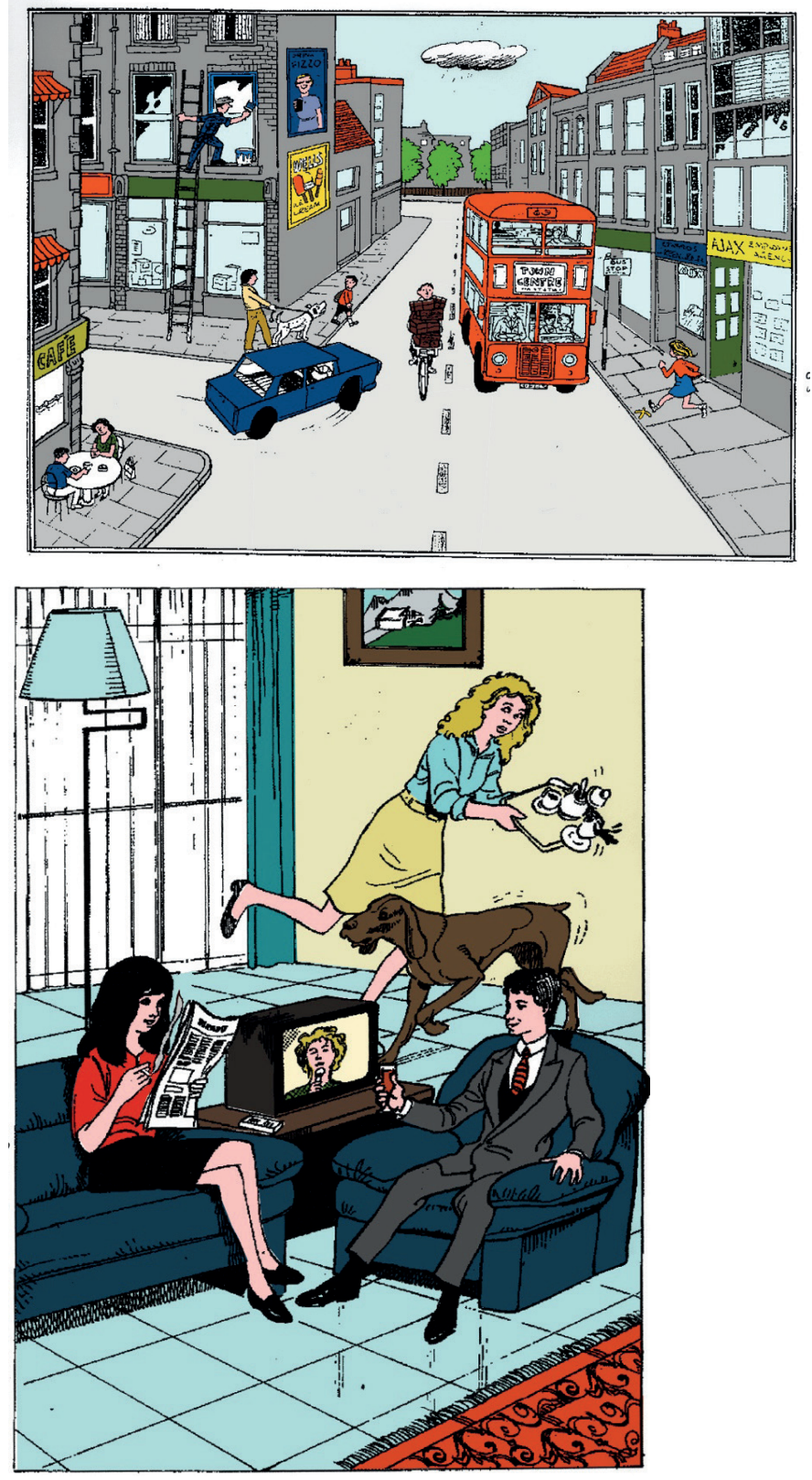

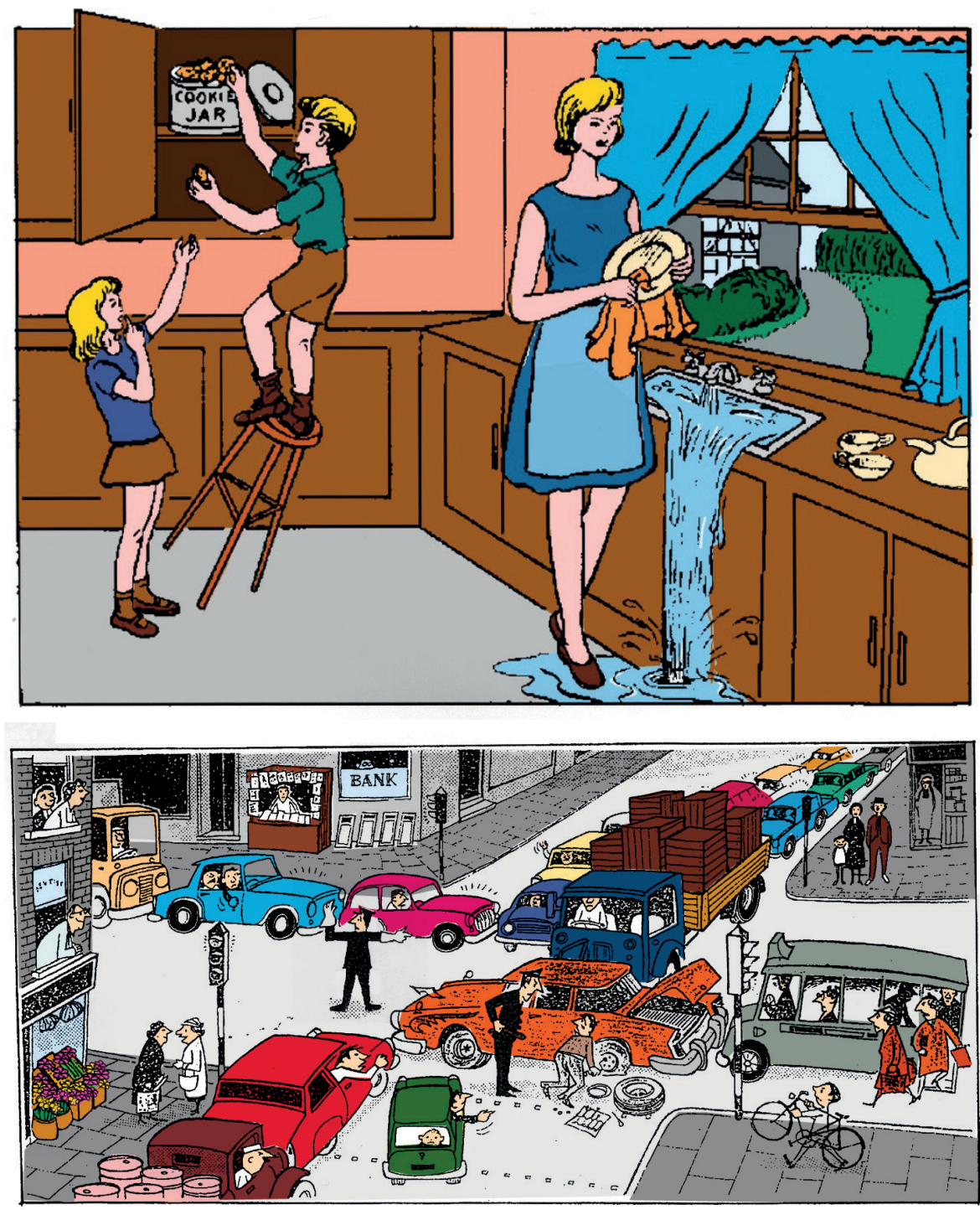


\section{Arousal Reappraisal Materials:}

\section{Intervention:}

\section{Thank you for taking part in this study!}

Engaging in the upcoming task can be a challenging experience. Before starting the task, we are going complete a short reading exercise. Specifically, in the following pages, you will be presented with summaries of scientific studies designed to help you perform well. After reading each study, we ask that you please write a short summary in your own words describing how the information presented can help you on upcoming task. 
This excerpt is adapted from Jamieson \& Mendes' 2010 study that appeared in the Journal of Experimental Psychology:

In challenging situations, people experience changes in their body. They might experience these as "unsettled feelings" or "butterflies in their stomach," and conclude that they are nervous. However, bodily changes that happen during this situation can be good. For instance, scientists have found that feelings of "butterflies" indicate that the body is gathering resources to meet situational demands.

Your bodily response is determined by our perceptions and beliefs. For example, imagine you are a skier staring down a steep slope with no other way off the mountain than going down this dangerous trail. Regardless of whether you like skiing, this situation is challenging. Expert skiers experience the this as "excitement" because they believe they can handle the difficult trail, whereas novices experience it as "fear" because the difficult trail exceeds their skill level. Thus, the skier's response (challenge vs. fear) depends on how they perceive the challenge.

Research about people with anxiety disorders indicates that these bodily reactions do not hurt performance, but can actually help because our brain releases chemicals that help us think quickly. So, during the task today, try to view your own bodily reactions as a coping tool.

In your own words please briefly describe how this information can help you perform well on the upcoming task:

This excerpt is adapted from Nock et al.'s (2011) study that appeared in the Journal of Clinical Psychology:

Arousal is a normal reaction that helps you face the challenges in your life. It is not harmful. In fact, if we did not have such bodily reactions we could not survive. If they are helpful, then why do most people see it as a negative experience? 
Research indicates that negative reactions to challenging situations like taking a test are the result of how we think about the situation (also known as 'cognitive appraisals'). When we experience something as challenging, our brain searches for possible sources of harm. However, in modern society there is often no physical threat. When no explanation can be found, the brain invents explanations such as, "There must be something wrong with me".

Nothing could be further from the truth. Increased heart rate, sweating, and heavy breathing are all signs that your body is delivering oxygen (fuel for thinking) to your brain. Bodily reactions to a challenge are adaptive and good.

In your own words please briefly describe how this information can help you perform well on the task today: 
The following is an illustrative diagram that shows the biological changes that happen when we experience a challenging situation. Please take a minute to note where the changes occur and how these help us do well.

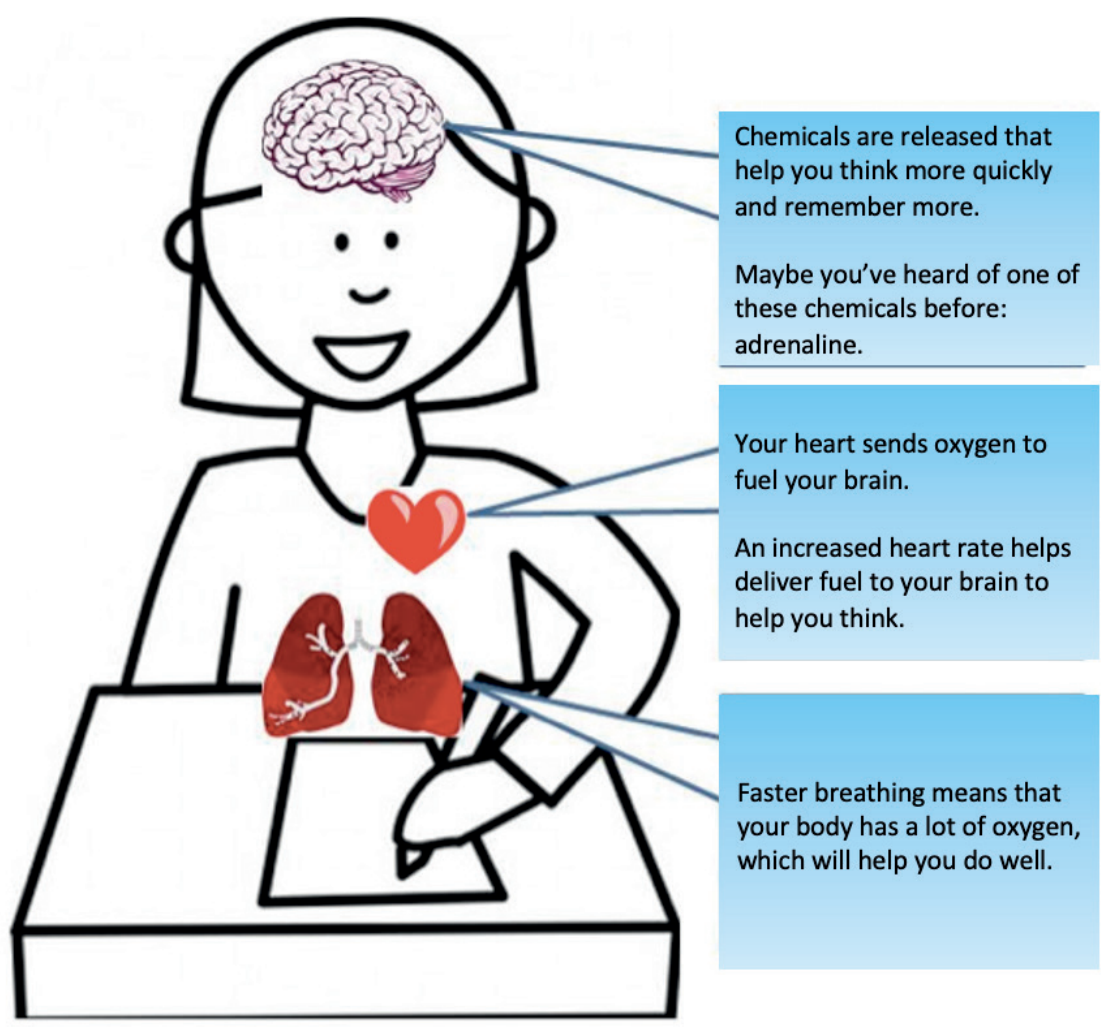

In your own words please briefly describe how this information can help you perform well on the upcoming task: 
Great job! You have finished the exercise. We would now like for you to answer some brief questionnaires before starting the task.

Remember during the upcoming task, we ask that you try to remind yourself that your body's responses to the testing situation will help you to perform well.

\section{Good luck!}




\section{Placebo:}

\section{Thank you for taking part in this study!}

Engaging in the upcoming task can be a challenging experience. Before starting the task, we are going complete a short reading exercise. Specifically, in the following pages, you will be presented with summaries of scientific studies designed to help you perform well. After reading each study, we ask that you please write a short summary in your own words describing how the information presented can help you on upcoming task. 
This excerpt is adapted from Jamieson \& Mendes' 2010 study that appeared in the Journal of Experimental Psychology:

Automatic thinking refers to the automatic thoughts people have in response to things happening around them. For instance, when faced with a challenging situation, an automatic thought might be "I don't know what to do". Our perceptions of the challenging situation make us believe that we are anxious.

Scientists have found that feelings of anxiety indicate that the individual is worried about a negative outcome. Moreover, they found that people with high levels of negative automatic thoughts are likely to respond to a challenging situation by feeling that their lives are less meaningful. This indicates that in order to have better mental health outcomes, one should reduce one's automatic negative thoughts. If one does not attend to whatever it is in the environment that causes negative thoughts, one will not experience anxiety. For instance, if someone is very afraid of spiders, he/she will not experience anxiety or fear if the spider is not noticed.

If you find yourself feeling anxious during the task today, remember that you will not feel as bad if you ignore these negative thoughts of anxiety. This will help you succeed in the upcoming task.

In your own words please briefly describe how this information can help you perform well on the task today: 
This excerpt is adapted from Nock et al.'s (2011) study that appeared in the Journal of Clinical Psychology:

The thoughts we experience in a challenging situation can be reduced by ignoring what is making us anxious. Ignoring these thoughts is not harmful. In fact, in modern society there are many potential sources of anxiety. To function in society, it is important to ignore negative thoughts by ignoring what makes us anxious.

Sometimes, we fail to ignore these negative thoughts. Ruminations about how we are going to perform can impair our ability to complete tasks because we devote cognitive resources to thinking about all the things that could go wrong. To reduce rumination, attention should be oriented inward and away from these thoughts. This helps us remain calm in the face of challenging situations.

If you find yourself experiencing anxiety during the upcoming test, try to put these thoughts out of your mind. Not thinking about the possible negative outcomes can help you remain calm during difficult testing situations.

In your own words please briefly describe how this information can help you perform well on the task today: 
The following is an illustrative diagram that shows how shifting attention away from arousal can help us perform well. Please take a minute to note where the changes occur and how these help us do well.

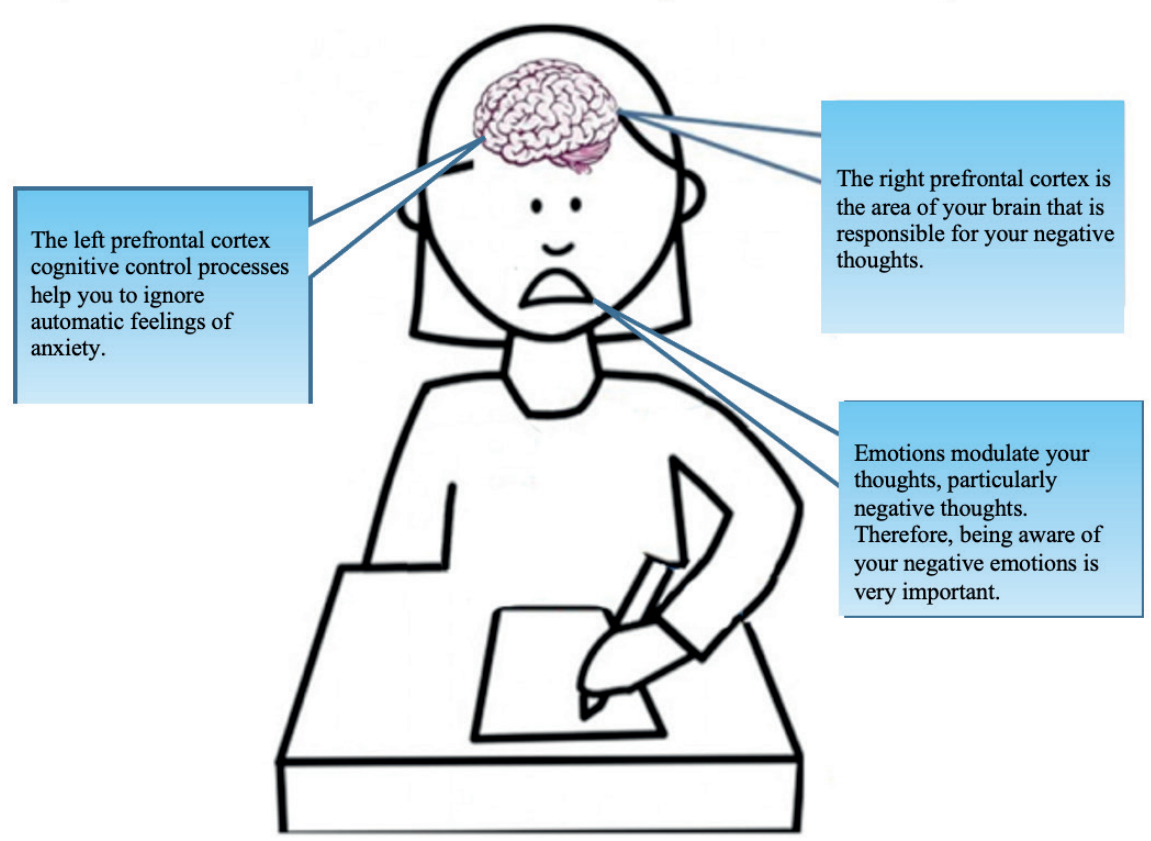

In your own words please briefly describe how this information can help you perform well on the task today: 
Great job! You have finished the exercise. We would now like for you to answer some brief questionnaires before starting the task.

Remember during the task today, we ask that you try to ignore anxious thoughts, while still fully complying with instructions given by the experimenter. This will help you to perform well.

\section{Good luck!}


Table C.A

Intraclass Correlation Coefficients for Free Recall Performance Coding

\begin{tabular}{lcccccc}
\hline & Intraclass & \multicolumn{3}{c}{$95 \%$ CI } & & \multirow{2}{*}{ Correlation } \\
\cline { 3 - 4 } & & Lower Bound & Upper Bound & $F$ & $p$ \\
\hline Traffic (Correct) & .892 & .814 & .937 & 18.883 & $<.001$ \\
\hline Traffic (Incorrect) & .680 & .496 & .804 & 5.654 & $<.001$ \\
\hline Kitchen (Correct) & .938 & .896 & .964 & 32.111 & $<.001$ \\
\hline Kitchen (Incorrect) & .736 & .561 & .843 & 7.312 & $<.001$ \\
\hline
\end{tabular}

Note. $N_{\text {free reclls }}=54(34 \%$ of total $) . N_{\text {coders }}=6$ (2 per language $) . \mathrm{CI}=$ confidence interval. Values < $.50=$ poor reliability; $.50-.75=$ moderate reliability; .75 to $.90=$ good reliability; $>.90=$ excellent reliability (Koo \& Li, 2016). 
Table C.B

Repeated Measures ANOVA Examining Demand and Coping Resources Separately

\begin{tabular}{lccccccccc}
\hline & Day 1 Pre-Enc & Pre-MAST & Post-Test & $\begin{array}{c}\text { Interaction } \\
\text { Timing x } \\
\text { Condition }\end{array}$ \\
& $M$ & $S D$ & $M$ & $S D$ & $M$ & $S D$ & & \\
\hline Task demands & & & & & & & & \\
Stress-Intervention & $3.720_{\mathrm{a}}$ & .908 & $5.198_{\mathrm{a}}$ & .668 & $4.585_{\mathrm{a}}$ & 1.219 & $F$ & $\mathbf{4 0 . 2 7 6}$ \\
Stress-Placebo & $3.517_{\mathrm{a}}$ & .984 & $4.658_{\mathrm{b}}$ & .957 & $4.119_{\mathrm{a}}$ & 1.076 & $p$ & $<.001$ \\
No-Stress-Placebo & $3.554_{\mathrm{a}}$ & .981 & $2.367_{\mathrm{c}}$ & 1.205 & $3.494_{\mathrm{b}}$ & 1.105 & $\eta_{p}{ }^{2}$ & $\mathbf{. 3 1 6}$ \\
\hline Coping resources & & & & & & & & \\
Stress-Intervention & $4.786_{\mathrm{a}}$ & .687 & $4.590_{\mathrm{a}}$ & .812 & $4.549_{\mathrm{a}}$ & 1.116 & $F$ & $\mathbf{4 . 3 0 8}$ \\
Stress-Placebo & $4.834_{\mathrm{a}}$ & .669 & $4.512_{\mathrm{a}}$ & 1.037 & $4.617_{\mathrm{a}}$ & .878 & $p$ & $\mathbf{. 0 0 2}$ \\
No-Stress-Placebo & $4.912_{\mathrm{a}}$ & .768 & $5.159_{\mathrm{b}}$ & .919 & $4.736_{\mathrm{a}}$ & .717 & $\eta_{p}{ }^{2}$ & $\mathbf{. 0 4 7}$ \\
\hline
\end{tabular}

Note . Enc $=$ encoding. MAST $=$ Maastricht Acute Stress Test. Test $=$ memory test. Stress appraisal questionnaire post-test was written in past tense. For both task demands and coping resources, the lowest possible score $=1$ (strongly disagree) and highest possible score $=7$ (strongly agree). Bold $=$ statistically significant at the $p<.05$ level. Values with different subscript letters in column are statistically significantly different at the $\mathrm{p}<.05$ level. 
Table C.C

Adjusted Descriptives and Inferential Statistics for Memory Performance Measures, Controlling for Baseline Negative Affect

\section{Stress-intervention Stress-placebo No-stress-placebo Stress effects}

\begin{tabular}{lccccccccc}
\hline & $M$ & $S D$ & $M$ & $S D$ & $M$ & $S D$ & $F$ & $p$ & $\eta_{p}^{2}$ \\
Free recall & & & & & & & & & \\
\hline Quantity & 70.878 & 20.587 & 72.499 & 20.443 & 69.323 & 20.314 & .318 & .728 & .004 \\
\hline Accuracy & .879 & .082 & .862 & .079 & .879 & .079 & .829 & .439 & .011 \\
\hline Recognition & & & & & & & & & \\
\hline Accuracy & .549 & .108 & .518 & .108 & .534 & .108 & 1.180 & .310 & .013 \\
\hline Sensitivity & -.034 & .722 & -.159 & .714 & .062 & .707 & 1.441 & .240 & .016 \\
\hline Response bias & .241 & .292 & .212 & .292 & .099 & .284 & $\mathbf{4 . 0 1 9}$ & $\mathbf{. 0 2 0}$ & $\mathbf{. 0 4 4}$ \\
\hline
\end{tabular}

Note. Reported results are from exploratory one-way ANCOVAs with condition (3 groups) as between-subjects factor and Baseline negative affect score as a covariate. Free recall quantity = number of total reported details. Free recall accuracy $=$ correct reported details/total reported details. Recognition accuracy $=$ correct answers/all questions. Recognition sensitivity $=d^{\prime}$. Recognition response bias $=c$. Findings that are statistically significant at the $p<.05$ level are in bold. 
Table C.D

Moderated Regression Analyses between Stress and Memory Performance Measures, Moderated by Dispositional Mindfulness

\begin{tabular}{|c|c|c|c|c|c|c|c|}
\hline & \multirow[t]{2}{*}{$R^{2}$} & \multirow[t]{2}{*}{$b$} & \multirow[t]{2}{*}{$S E$} & \multirow[t]{2}{*}{$t(3,173)$} & \multicolumn{2}{|c|}{$95 \% \mathrm{CI}$} & \multirow[t]{2}{*}{$p$} \\
\hline & & & & & lower & upper & \\
\hline Recall Quantity & .021 & & & & & & \\
\hline Stress & & 6.582 & 17.045 & .386 & -27.089 & 40.254 & .700 \\
\hline Mindfulness & & .319 & .283 & 1.129 & -.239 & .877 & .261 \\
\hline Interaction & & -.060 & .134 & -.449 & -.324 & .204 & .654 \\
\hline Recall Accuracy & .003 & & & & & & \\
\hline Stress & & .025 & .067 & .364 & -.108 & .157 & .716 \\
\hline Mindfulness & & .001 & .001 & .519 & -.002 & .003 & .604 \\
\hline Interaction & & -.001 & .001 & -.351 & -.001 & .001 & .726 \\
\hline Recognition Accuracy & .008 & & & & & & \\
\hline Stress & & .073 & .088 & .832 & -.099 & .245 & .407 \\
\hline Mindfulness & & .001 & .002 & .735 & -.002 & .004 & .464 \\
\hline Interaction & & -.001 & .001 & -.913 & -.002 & .001 & .362 \\
\hline $\begin{array}{l}\text { Recognition } \\
\text { Sensitivity }\end{array}$ & .003 & & & & & & \\
\hline Stress & & -.004 & .580 & -.007 & -1.148 & 1.141 & .995 \\
\hline Mindfulness & & -.001 & .010 & -.067 & -.020 & .019 & .947 \\
\hline Interaction & & .001 & .005 & .085 & -.009 & .009 & .932 \\
\hline
\end{tabular}

Note. $\mathrm{CI}=$ confidence interval . 
